PROGRAMA DE DOCTORADO EN INGENIERÍA INDUSTRIAL

\author{
TESIS DOCTORAL:
}

\title{
SIMULATION AND OPTIMIZATION METHODS AS DECISION SUPPORT TOOLS FOR OPERATION OF OIL REFINERY HYDROGEN NETWORKS
}

Presentada por Aníbal Santiago Galán Prado para optar al grado de Doctor/a por la Universidad de Valladolid

Dirigida por:

D. César de Prada Moraga

Dña. Gloria Gutiérrez Rodríguez 


\section{AgradeCimientos}

Financial support is gratefully acknowledged from the Marie Curie Horizon 2020 EID-ITN project "PROcess NeTwork Optimization for efficient and sustainable operation of Europe's process industries taking machinery condition and process performance into account - PRONTO", Grant agreement No 675215. I am thankful to all supervisors and researchers within PRONTO for their support, collaboration and for being an endless source of motivation throughout my PhD journey.

Ahora voy a seguir en español...

Mis más sinceros agradecimientos a mis directores de tesis, Gloria y César, que confiaron mí para llevar adelante este trabajo. Quisiera agradecerles especialmente su disponibilidad incondicional y generosidad a la hora de compartir sus conocimientos, que ha sido una fuente de inspiración y motivación en estos cuatro años.

A mis compañeros de la sala de investigadores con los que compartimos muchísimas horas de trabajo, pero también tuvimos tiempos de recreo y celebración. No los nombro porque no quiero omitir a ninguno. Igualmente a los profesores y técnicos de laboratorio del ISA en la sede Mergelina por su apoyo y compañía. A Daniel, que desde Burgos también se hacía tiempo para tener charlas sobre redes de hidrógeno y el OptimH2.

A todos los compañeros y colegas en Petronor, especialmente a Rafa por confiar en mí para encarar este trabajo que se convirtió en una grandiosa aventura. Gracias a todo el departamento de Optimización y Control Avanzado por su acogida, por los miles de cafés, comidas, recetas, reuniones y charlas compartidas. Tampoco los voy nombrar porque no quiero omitir a nadie, pero les agradezco de todo corazón. Sin saberlo, se transformaron en mi familia en Euskadi.

Sin dudas, estaré eternamente agradecido con el Prof. Ignacio Grossmann y su grupo de investigación en Carnegie Mellon University (CMU), Pittsburgh, USA, y todos los 
investigadores del Center for Advanced Process Decision-Making (CAPD) en CMU, quienes no solo me recibieron en su grupo estupendamente, si no que las conversaciones e intercambios durante mi estancia allí inspiraron varias secciones de esta tesis. Espero ansioso que la vida nos vuelva a juntar pronto.

Claramente, tengo que agradecer a mis amigos de Uruguay que con más de 25 años de amistad encima, somos prácticamente hermanos. Definitivamente, esta experiencia doctoral en el extranjero no habría sido lo mismo sin ellos del otro lado del WhatsApp.

A mis padres que siempre me dieron su apoyo y estimularon para derribar barreras y mejorar. Obviamente a mi madre que me muestra todos los días cómo ir a por más. También agradezco a mis hermanos, Diana y Mauri, porque aunque estemos lejos físicamente siempre están cerca en espíritu. Obviamente a Amaia que nos iluminó la vida a todos. Hago extensivo el agradecimiento al resto de mi familia, mis tíos, primos y sobrinos.

A Fer que eligió encarar conmigo estos años lejos de nuestras familias y que me sigue acompañando en todas las aventuras desde hace 15 años. Pienso igual que esto recién empieza y que lo mejor siempre es una sorpresa que está por venir. 


\section{TABLE OF CONTENTS}

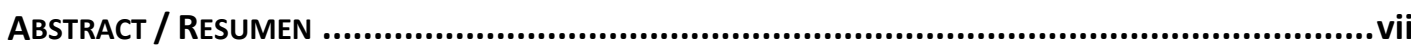

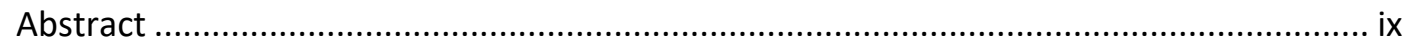

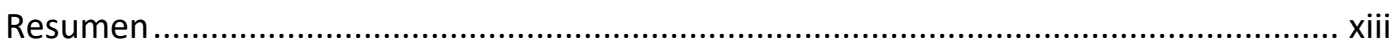

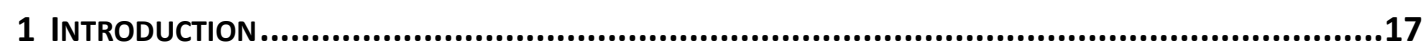

1.1 Overview of decision support in the process industry ......................................... 19

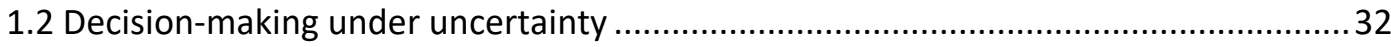

1.3 Hydrogen networks of crude oil refineries..................................................... 39

1.4 Case study motivation ................................................................................ 51

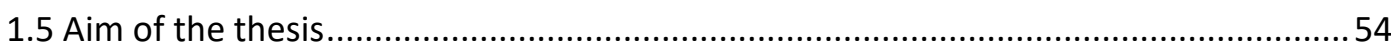

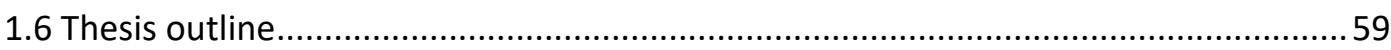

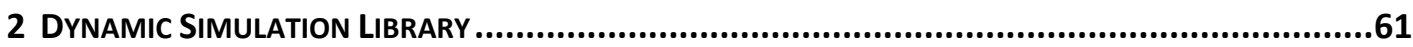

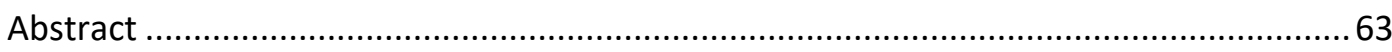

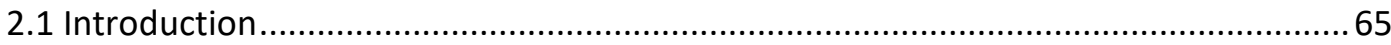

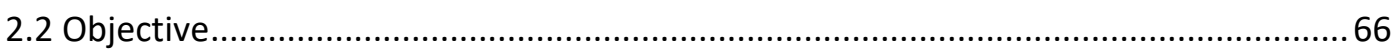

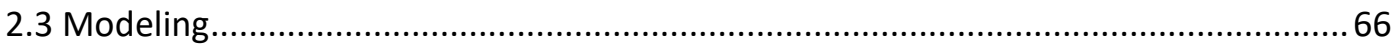

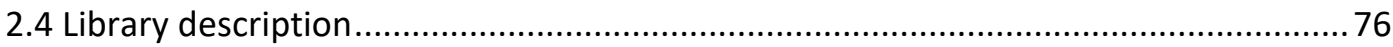

2.5 Library utilization and validation process...................................................... 101

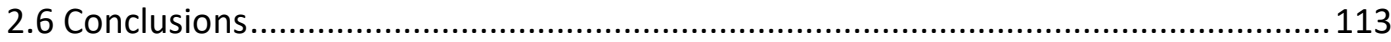

3 REAL-TIME RECONCILED SIMULATION ................................................................115

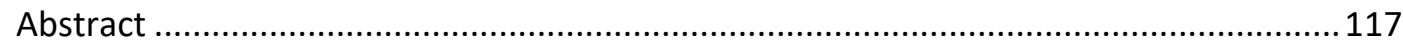

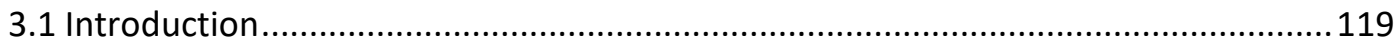




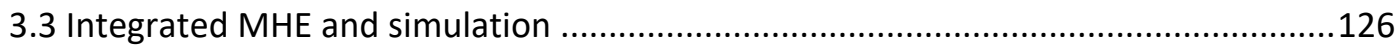

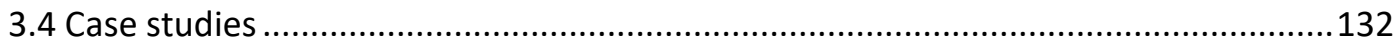

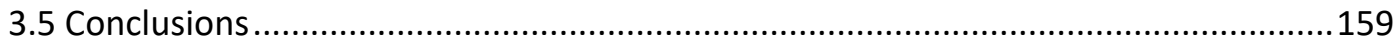

4 OPTIMIZATION UNDER UNCERTAINTY............................................................. 161

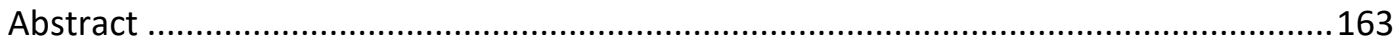

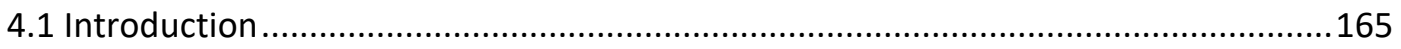

4.3 Incorporation of explicit uncertainty ....................................................... 187

4.4 Description of the TSSP problem .................................................................... 189

4.5 Risk neutral problem statement................................................................ 193

4.6 Evaluation of the value of the stochastic solution ............................................ 194

4.7 Considering risk in the decision making process ..............................................199

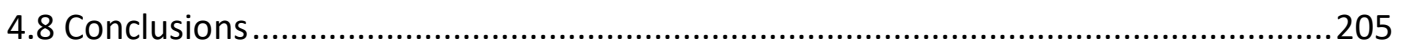

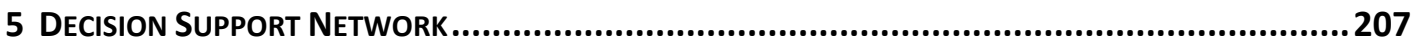

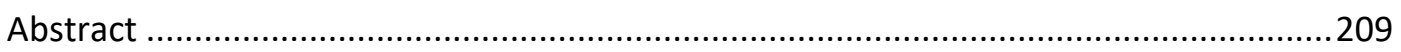

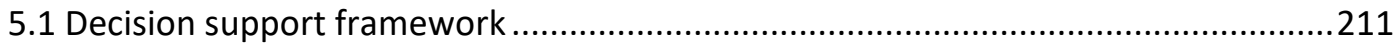

5.2 Design of a decision support framework...........................................................218

5.3 Enterprise-wide decision support framework .................................................221

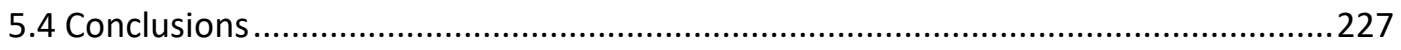

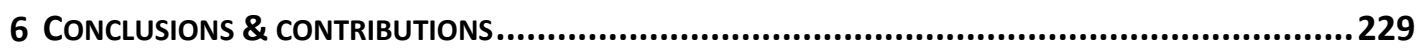

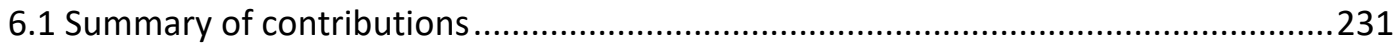

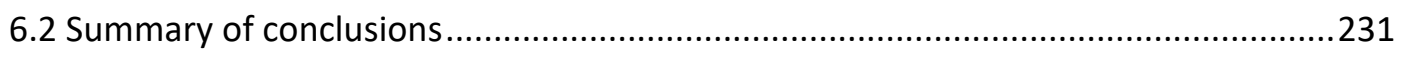

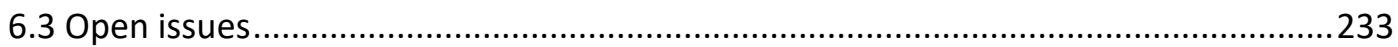




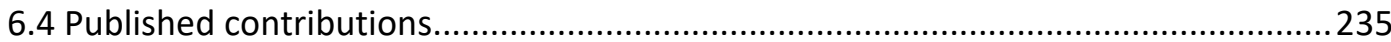

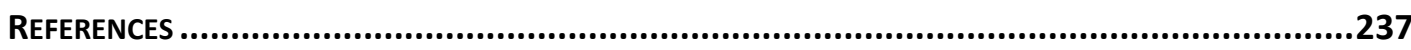

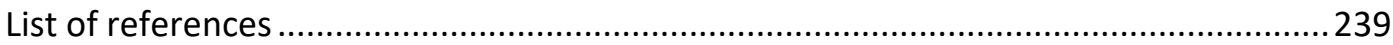

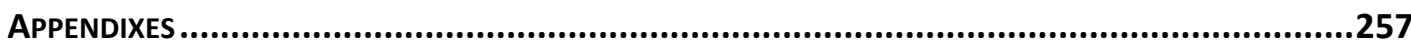

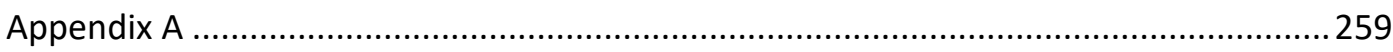

A.1 Simulation and optimization foundations.................................................... 259

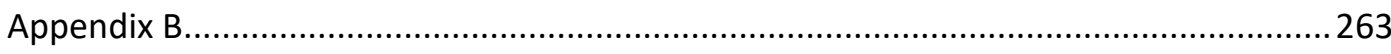

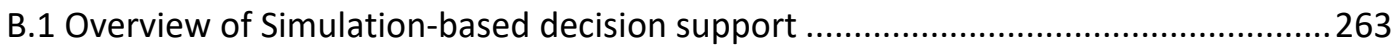

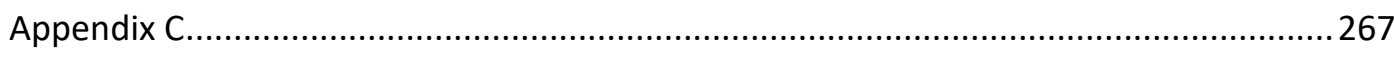

C.1 Notes on optimization-based decision support ........................................... 267

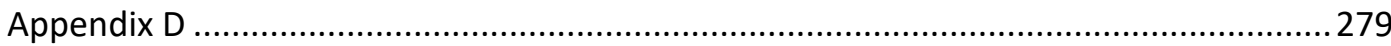

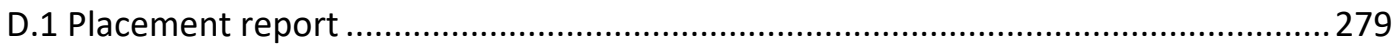



ABstract / RESUMEN 



\section{AbStract}

The process industry has been at the forefront of manufacturing technology advances, systematically aiming for sustainable process improvements. Generally, improvements come as a result of decision-making processes which may be backed on information from various sources. In fact, decision making in manufacturing operations is indeed complex, as it accounts for different scopes and timescales depending on the aimed activities ranging from strategic business management decisions (long term decisions) to basic regulatory control of the process (automatic actions). Despite that, actual deployment of integrated decision support systems remains atypical in process industries, which constraints the potential of optimal operation of assets. This thesis focuses primarily on advanced and supervisory control tools, which typically translates into plant-wide decisions at timescales from minutes (advanced control) to hours (supervisory control).

In this context, this thesis centers the discussion on crude oil refinery hydrogen networks operations, especially considering change of condition mitigation measures to support operators' decision-making process. Hydrogen in oil refineries is used for sulfur removal from intermediate products to produce commercial fuels (e.g.: diesel, gasoline) and increase crude oil yields. Therefore, refinery hydrogen networks operation demands maximization of process units loads (benefits) minimizing hydrogen production (costs), subjected to uncertainties of the equipment and bounded to operational and safety constraints. In particular, this thesis uses Petronor refinery in Bilbao, Spain, as case study.

The main aim of this thesis is to study and develop a decision support tool for refinery hydrogen network operators. For this purpose, it is proposed an integrated decision support framework for hydrogen network operators under uncertain conditions, which combines process information, model predictive control, optimization and simulation tools. 
Firstly, a first principles hydrogen network dynamic simulation library is described and discussed towards its utilization as the backbone of decision support tools with focus on the architecture. Furthermore, an example of architecture is proposed and analyzed towards the use of this library for developing enhanced simulation environments. The discussion ends with thoughts, based on an example, about leveraging dynamic simulation into real-time operation environments.

Secondly, a simulation-based decision support tool called real time reconciled simulation is introduced. The study describes real-time reconciled simulation (RTRS), and analyzes its usefulness as decision-making tool for process operators, especially under unexpected process changes. The proposed methodology and architecture is implemented in two case studies in the context of an oil refinery hydrogen network, both plant and network levels are considered. A what-if analysis is conducted on case studies, assessing two feasible mitigation actions for each case baseline condition. The focus of the discussion is, nevertheless, on the methodology itself and its general features as decision support tool. It is highlighted the fact that RTRS complements in a straightforward manner other control operation tools such as model predictive controllers (MPC) and real-time optimizers (RTO). Therefore, it may add to any decision support framework an open-loop component with parameter estimation and forecasting capabilities. Moreover, its potential for training and integration within other tools packages is discussed.

Thirdly, the problems associated with the implementation of a real-time optimization (RTO) decision support tool, for the operation of a large scale hydrogen network of an oil refinery is addressed. In addition, a formulation which takes into account the stochastic uncertainty of hydrogen demand, due to hydrocarbons quality change, is described and further studied, focusing on its utility in the decision-making process of operators. An integrated robust data reconciliation, and economic optimization, considering plant-wide uncertain parameters is presented and discussed. Moreover, stochastic uncertainty in hydrogen demand is assessed for its inclusion within the RTO framework. A novel approach of the decisions stages at hydrogen producers and 
consumers is proposed, which supports the formulation of the problem as a two-stage stochastic non-linear program. Representative results are presented and discussed, aimed at assessing the potential impact in the hydrogen management policies. For this purpose, the value of the stochastic solution, perfect information, and expectation of the expected value are analyzed. Complementarily, a risk-averse formulation is presented (value-at-risk and conditional-value-at-risk) and its results compared against the risk-neutral formulation.

Fourthly, the integration of multiple decision support tools is presented and discussed in the context of what is named decision support frameworks (DSFs). These are the natural environment of decision-making tools, however in practice these tools reside in different silos across multiple systems within company. This configuration challenges information and data exchange amongst tools in a transparent manner, which can result in inconsistent solutions due to different applications using different data sets for the same purpose. In order to address these issues and provide enhanced decision-making support across operations, a DSF architecture is proposed and studied.

The DSF architecture features previously presented tools such as RTO and RTRS, while it introduces the data management system role, the digital twin role, online and offline simulation and other features. The discussion is focused on how the architecture would improve decision makers' capacity of making complex decisions supported by updated information from across the business, with especial interest in process operations. Furthermore, DSFs promote enhanced process knowledge and skills transfer, due to their ease-of-access to consistent information along with their forecasting and assessment capability over multiple operation alternatives (e.g. What-if analysis). In addition, the proposed DSF architecture considers tailored models, which are supported by the library of models under the scope of the digital twin.

The final section of this thesis consists of a summary of the conclusions of each section, along with future challenges and open issues going forward. This section ends with a list of publications and contributions of this thesis. 



\section{RESUMEN}

La industria de procesos ha estado a la vanguardia de los avances en la tecnología de fabricación, buscando sistemáticamente mejoras sostenibles en los procesos. Generalmente las mejoras son el resultado de procesos de toma de decisiones que pueden estar respaldados por información de diversas fuentes. En los hechos, la toma de decisiones operativas en procesos productivos es compleja, ya que tiene en cuenta diferentes alcances y escalas temporales según las actividades a las que se destine, estas pueden ir desde las decisiones estratégicas de gestión empresarial (largo plazo) hasta el control básico del proceso (acciones automáticas). A pesar de ello, la utilización real de sistemas integrados de apoyo a la toma de decisiones sigue siendo atípica en las industrias de procesos, lo que limita las posibilidades de un funcionamiento óptimo de las unidades de proceso. Esta tesis se centra principalmente en las herramientas de control avanzado y de supervisión, lo que normalmente se traduce en decisiones a nivel de planta completa en escalas de tiempo que van desde minutos (control avanzado) hasta horas (control de supervisión).

En este contexto, la tesis centra la discusión en las operaciones de las redes de hidrógeno de las refinerías de petróleo crudo, especialmente considerando las medidas de mitigación de cambio de condición para apoyar el proceso de toma de decisiones de los operadores. El hidrógeno en las refinerías de petróleo se utiliza para la eliminación del azufre de los productos intermedios para producir combustibles comerciales (por ejemplo: diesel, gasolina) y aumentar el rendimiento de productos refinados del petróleo crudo. Por consiguiente, el funcionamiento de las redes de hidrógeno de las refinerías exige la maximización de las cargas de las unidades de proceso (beneficios) minimizando la producción de hidrógeno (costos), sujeta a las incertidumbres de equipos y a las limitaciones operacionales y de seguridad. En particular, en esta tesis se utiliza como caso de estudio la refinería de Petronor en Bilbao (España). 
El objetivo principal de esta tesis es estudiar y desarrollar una herramienta de apoyo a la toma de decisiones para los operadores de redes de hidrógeno. Con este fin, se propone un marco integrado de apoyo a la toma de decisiones para los operadores de redes de hidrógeno considerando incertidumbres, de modo que se combina información sobre los procesos, control predictivo basado en modelos, optimización y herramientas de simulación.

En primer lugar, se describe y examina una biblioteca de simulación dinámica de redes de hidrógeno de primeros principios con miras a su utilización como columna vertebral de las herramientas de apoyo a la toma de decisiones, centrándose en el estudio de su arquitectura. Además, se propone y analiza un ejemplo de arquitectura con miras a la utilización de esta biblioteca para el desarrollo de entornos de simulación mejorados. La discusión finaliza con reflexiones, basadas en un ejemplo sobre el aprovechamiento de la simulación dinámica en entornos de funcionamiento en tiempo real.

En segundo lugar, se presenta una herramienta de apoyo a la toma de decisiones basada en simulación, llamada simulación reconciliada en tiempo real. En el estudio se describe la simulación reconciliada en tiempo real (RTRS) y se analiza su utilidad como herramienta para la toma de decisiones para operadores de procesos, especialmente en caso de cambios inesperados. La metodología y la arquitectura propuestas se aplican en dos casos de estudio en el contexto de una red de hidrógeno de una refinería de petróleo; se consideran tanto los niveles de planta individual como los toda la red. En los casos de estudio se lleva a cabo un análisis "Qué pasa si", en el que se evalúan dos medidas de mitigación viables para cada condición de base del caso. No obstante, la discusión se centra en la metodología propiamente dicha y sus características generales como soporte en la toma de decisiones. Se destaca el hecho de que el RTRS complementa de manera directa otras herramientas de control operativo, como los controladores predictivos de basados en modelos (MPC) y la optimización en tiempo real (RTO). Por lo tanto, puede incorporar a cualquier marco de apoyo a la toma de decisiones un componente en lazo abierto con capacidades de estimación de parámetros y de previsión. Además, se discute 
su uso como herramienta de capacitación, así como su integración con otros paquetes de herramientas.

En tercer lugar, se abordan los problemas relacionados con la aplicación de una herramienta de apoyo a la toma de decisiones de optimización en tiempo real para el funcionamiento de una red de hidrógeno de una refinería de petróleo. Además, se describe y se estudia en detalle una formulación que tiene en cuenta la incertidumbre estocástica de la demanda de hidrógeno, debido al cambio de calidad de los hidrocarburos, centrándose en su utilidad en el proceso de toma de decisiones de los operadores. Se presenta y se analiza una reconciliación integrada y sólida de los datos, así como la optimización económica, teniendo en cuenta los parámetros inciertos a nivel de toda la planta. Además, se evalúa la incertidumbre estocástica en la demanda de hidrógeno para su inclusión en el marco del RTO. Se propone un enfoque novedoso de las etapas de decisión en los productores y consumidores de hidrógeno, que respalda la formulación del problema como una programación no lineal y estocástica de dos etapas. Se presentan y discuten resultados representativos, con el fin de evaluar el posible impacto en las políticas de gestión del hidrógeno. Para ello se analiza el valor de la solución estocástica (VSS), la información perfecta y la expectativa del valor esperado. Complementariamente, se presenta una formulación con aversión al riesgo (valor en riesgo y valor condicional en riesgo) y se comparan sus resultados con la formulación de riesgo neutral.

En cuarto lugar, la integración de los instrumentos de apoyo a las decisiones múltiples se presenta y examina en el contexto de lo que se denomina marcos de apoyo a la toma de decisiones (DSFs). Éstos son el entorno natural de las herramientas de toma de decisiones, sin embargo en la práctica estas herramientas residen en diferentes silos dispersas en múltiples sistemas dentro de la empresa. Esta configuración dificulta el intercambio de información y datos entre las herramientas de manera transparente, lo que puede dar lugar a soluciones incoherentes debido a que las diferentes aplicaciones utilizan diferentes conjuntos de datos para el mismo propósito. Con el fin de abordar estas 
cuestiones y proporcionar un mejor apoyo a la toma de decisiones en todas las operaciones, se propone y estudia una arquitectura de DSF.

La arquitectura del DSF presenta herramientas previamente presentadas, como el RTO y el RTRS, a la vez que introduce el papel del sistema de gestión de datos, el papel del gemelo digital, la simulación en línea y fuera de línea y otras funcionalidades. El análisis se centra en cómo la arquitectura mejoraría la capacidad de los encargados de adoptar decisiones complejas con el respaldo de información actualizada de toda la empresa, con especial interés en las operaciones de los procesos productivos. Además, los marcos estratégicos de desarrollo promueven una mayor transferencia de conocimientos y aptitudes en materia de procesos, debido a su facilidad de acceso a información consistente junto con su capacidad de previsión y evaluación sobre múltiples alternativas de funcionamiento (por ejemplo, el análisis "Qué pasa si"). Asimismo, en la arquitectura propuesta de los marcos digitales de referencia se tienen en cuenta los modelos adaptados, que se apoyan en la biblioteca de modelos en el ámbito del gemelo digital.

La sección final de esta tesis consiste en un resumen de las conclusiones de cada sección, junto con los retos y temas pendientes de cara al futuro. Esta sección termina con una lista de publicaciones y contribuciones de esta tesis. 


\section{INTRODUCTION}





\subsection{OVERVIEW OF DECISION SUPPORT IN THE PROCESS INDUSTRY}

The process industry has been at the forefront of manufacturing technology advances, systematically aiming for sustainable process improvements. Most likely, improvements come as a result of decision-making processes which may be backed on information of various sources. This has been addressed by the process operations community as early as in 2003 as commented by Grossmann and McDonald (2004). Process systems engineering (PSE) has been one major contributor to this progress since early days of computer-aided engineering (Doherty et al., 2016; Sargent, 1967) until nowadays (Ignacio E. Grossmann and Harjunkoski, 2019). The extended use of process simulation in plant design, especially in complex systems is an example decision-making support utilizing PSE techniques. Similarly, nowadays production scheduling decisions are based on some sort of mixed integer linear programming (MILP) problem resolution ${ }^{1}$, which after being correctly analysed provides key information to decision makers. Thereby, the MILP problem is seen as the optimization method used for decision support of scheduling production plans. Likewise, decisions across a particular business or industrial asset heavily depend on their decision support tools. In general terms a decision support system (DSS) can be defined as, "interactive computer-based systems, which help decision makers utilize data and models to solve unstructured problems" (Sprague, 1980). Another simplified and less restrictive definition of DSS can refer to any unbiased information system used in the decision-making process. Intuitively speaking, it is implicit the systematic approach to support decision-making processes which is underpinned in data analysis, simulation and optimization methods. Refer to (Benbasat and Nault, 1990; Sprague, 1980) and the references therein for some seminal work on DSS.

In the following subsections I review the main aspects of decision support systems in manufacturing operations. The focus is on the main structure, identifying the PSE

\footnotetext{
${ }^{1}$ Refer to Appendix A for a brief introduction on simulation and optimization foundations.
} 
methods utilized in addressing this problem and the interactions across operations. The methods themselves are reviewed separately in subsequent sections.

\subsubsection{DECISION-MAKING PROCESS IN MANUFACTURING OPERATIONS}

Decision making in manufacturing operations is indeed complex, as it accounts for different scopes and timescales depending on the aimed activities ranging from strategic business management decisions (long term decisions) to basic regulatory control of the process (automatic actions) as portrayed in Fig. 1.1. Similarly, this topic has been addressed from a supply chain standpoint, leading to the so called enterprise-wide optimization (EWO) as introduced by Grossmann (2005) and his research group in the Center for Advanced Process Decision Making at Carnegie Mellon University in Pittsburgh, USA. Although DSS are widely extended in the process industry, their integration across activities in an organization has been challenging and remains limited. For instance, if manufacturing decisions are considered at all five levels represented in the international standard ANSI/ISA-95.00.01-2000 (2000) and ANSI/ISA-95.00.03-2005 (2005), then the following operations sit at different levels of the control pyramid, see Fig. 1.1:

- level four - business management, planning,

- level three - scheduling, real-time optimization,

- level two - advanced and predictive control,

- level one - basic control and sensing,

- level zero - physical production process. 


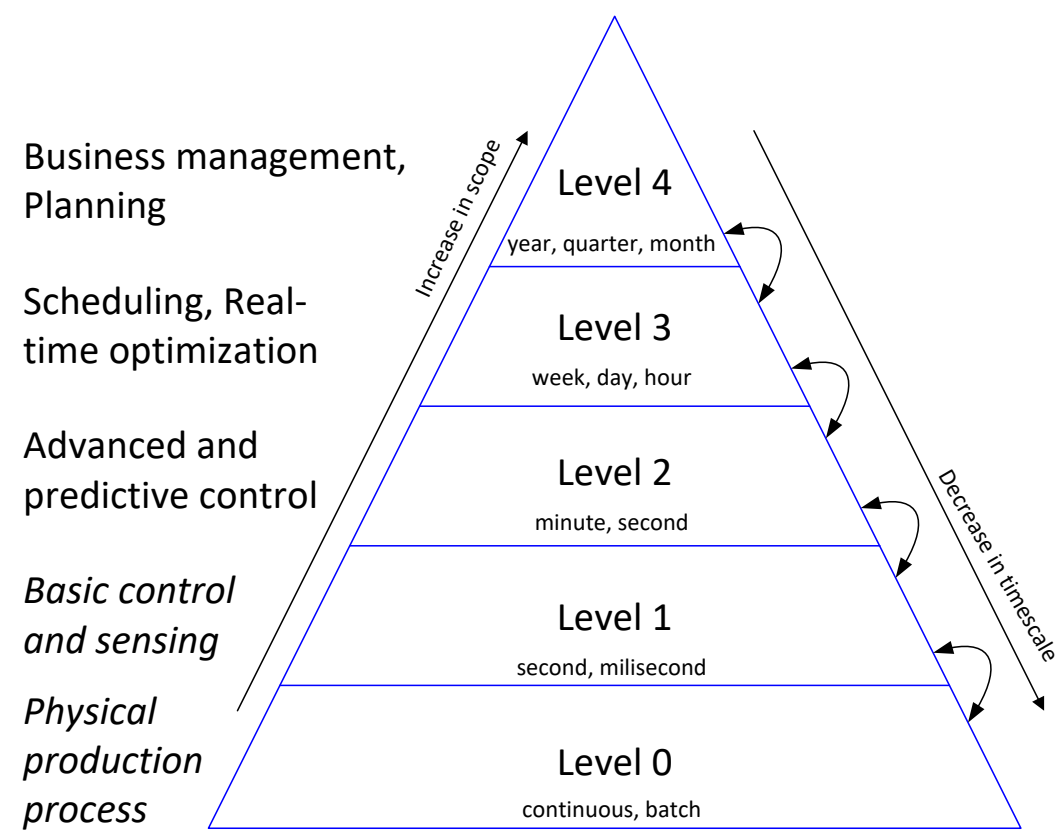

Fig. 1.1 - Manufacturing operations control pyramid based on ANSI/ISA-95.00.03-2005 (2005).

Level four, scopes enterprise-wide decisions such as production or investment planning. These decisions are typically in a timescale of months, quarters, or years, depending on the business and specific aim. Decision systems at this level are named enterprise resource planning (ERP), typically comprising of a suite of integrated software tools. Mixed integer linear programming (MILP) is the most common approach when dealing with enterprise-wide problems. In essence, effective linearization of complex systems and their integration is generally the main challenge seen at this level, especially due to inaccurate representation of process nonlinearities. Although a detailed review of enterprise-wide optimization and supply chain methods is outside the scope of this thesis, introductory notes on this research field are provided for reference in Appendix C.

Level three, scopes decisions in a timescale range of hours up to weeks such as scheduling and real-time optimization. Decision systems at the top of this level are normally named manufacturing execution systems (MES), which support manufacturing operation management (MOM). Mixed integer linear programming (MILP), mixed integer 
nonlinear programming ${ }^{2}$ (MINLP) or nonlinear programming (NLP), can be suitable for scheduling (most likely MILP or MINLP) and real-time optimization (most likely MINLP or NLP), however, the lower the detail the higher the prevalence of MILP over MINLP. Therefore, MILP is generally dominant in scheduling applications (timescale of weeks or days) whereas MINLP or NLP is dominant in real-time optimization applications (timescale of hours). It is important to bear in mind that suitable process representation goes alongside timescales, thereby, nonlinearities neglected in timescales of days or weeks are typically required when timescale granularity is in the range of hours. Although a detailed review of scheduling techniques is outside the scope of this thesis, summary notes about this research field are provided for reference in Appendix C. Real-time optimization is addressed in section 1.1.3 Integration of simulation and optimization in decision support systems.

Level two, scopes decisions in a timescale range of minutes up to seconds such as those of advanced and predictive control. These are in effect a supervisory layer over basic control and do not necessarily consider process economy in their control law. Instead, the focus is on process stability and the capability of tracking reference trajectories. It is generally accepted that NLP formulations should perform better than LP formulations. Nonetheless, LP still has an important share of total implementations in the industrial sector due to the fact that for the same optimization problem a LP formulation is more robust than a NLP formulation, and ensures absolute optimum rather than local optimum. At this level, process dynamics and wall clock time are of key importance and are accounted in the models and formulations.

Level one, scopes decisions in a timescale range of seconds up to miliseconds such as those required for regulatory control. At this level, is where actuators and sensors operate as well as control loops are used to adjusting control valves to meet a given set point. No process economy criterion is used in the tuning at this level, being the aim to minimize off set of the process value with respect to a reference set point at steady state.

\footnotetext{
${ }^{2}$ Refer to Appendix A for a brief introduction on simulation and optimization foundations
} 
Level zero, implies the process itself so there is no formal decision at this level.

\subsubsection{OVERVIEW OF DECISION-MAKING INTEGRATION ACROSS LEVELS}

All levels have information exchange within them, being outputs of some levels used as inputs of others and vice-versa, see the arrows in Fig. 1.1. Additionally, each level consists of several applications that are running alongside each other and exchanging information. In particular, information exchange is a common bottleneck that challenges efficient optimization of decisions across levels. As an example, the process economy is addressed differently in levels: four, three and two. Therefore, additional benefit would arise from the effective integration of these three levels.

Authors such as Prades et al. (2013) analyze the relevance of level four and three integration in compliance with ANSI/ISA-95.00.03-2005 (2005) standard for business process models applying Business Process Modeling Notation (BPMN) language. While another part of the research community focuses on modular, somehow horizontal decision support framework (DSF), where a common ground of interactions space is used to bridge the gaps between individual sections (Cheng et al., 1999; Rolandi and Romagnoli, 2010; Vrabič et al., 2018). This concept has been extended to digital twins (Haag and Anderl, 2018; Stark et al., 2019; Vrabič et al., 2018) and smart factory (Li, 2016), where the connexion aims to match models with actual assets ultimately generating virtual plants.

\subsubsection{INTEGRATION OF SIMULATION AND OPTIMIZATION IN DECISION SUPPORT SYSTEMS}

Primarily, it should be noted that simulation and optimization integration into decision support systems for manufacturing processes has received limited attention from the academic and industrial sectors, to the best of my knowledge. Interest has been given to DSS, simulation and optimization applications as standalone tools, rather than considering these as coordinated pieces of a DSS with a common goal. Consequently, there is limited literature addressing these three dimensions of decision support systems. Nonetheless, it is important to understand how best leverage optimization and simulation 
in a DSS architecture. This point is addressed in chapter five, when describing the decision support framework architecture.

Optimization and simulation methods are suitable for decision-making processes across levels. Appropriate combination of optimization and simulation tools enables accurate process forecasting (simulation), while help optimize process economy (objective function) subject to a set of constraints (boundaries). Nonetheless, most DSS rather than integrating simulation and optimization may focus on one or the other depending on the specific application. Poor integration of simulation and optimization is particularly true when addressing problems across levels in the control pyramid (Fig. 1.1), as it is pointed out by Grossmann (2005) and Perea-López et al. (2003). A more detailed discussion on the integration across levels and its challenges is addressed by Harjunkoski et al. (2009) and Baldea and Harjunkoski (2014). Although integration across levels is challenging, it is recognized as promising due to its potential towards optimal operation (Engell and Harjunkoski, 2012; Grossmann, 2012). Other authors focus their analysis on the integration from a point of view of the manufacturing execution system (MES), which essentially resides at level three, Saenz de Ugarte et al. (2009) provides a thorough literature review in reference to this topic, which in essence, is valid to this day. In particular, an interesting description of MES framework is studied by Cheng et al. (1999), this was an early contribution in the MES development field combining object-oriented software with a modularized and distributed architecture.

Another important perspective to bear in mind is regarding which tools are present in DSS, especially how these contribute in aiding in decision making. Moreover, it is critical to understand the purpose, techniques and outputs of individual applications that exchange information with particular DSS in order to analyse the usefulness of the DSS itself. Therefore, it is critical to understand the optimization methods used in tools helping manufacturing decision making, such as planning, scheduling, real-time optimization (RTO) and advanced control. For instance, in this thesis the focus is primarily on levels 2 and 3 , which are in the scope of RTO and model predictive control (MPC). In the following section 
I provide a succinct overview of MPC and RTO. An expanded version of these overview notes on MPC and RTO are provided for reference in Appendix C.

\subsubsection{MODEL PREDICTIVE CONTROL}

MPC is a form of control based on model predictions, in which the current control action is obtained by solving on-line, at each sampling time, a finite horizon open-loop optimal control problem, using the current state of the plant as the initial state. The result of the optimization is an optimal control sequence and the first control action in this sequence is applied to the plant. For general introduction on MPC fundamentals and reviews I would recommend the following literature: Camacho and Bordons (2007), García et al. (1989), Lee (2011), Mayne et al. (2000), Morari and Lee (2014).

The key idea of MPC was the utilization of a model to optimize the control actions along a moving horizon, such that, target trajectories of controlled variables (CV) are as close as possible to predicted outputs of $\mathrm{CV}$, while being subjected to a set of constraints (Fig 1.2). This approach was transformative in the field of advanced control in the process industry at the same time that gained popularity in the academic sector (Lee, 2011; Morari and Lee, 2014).

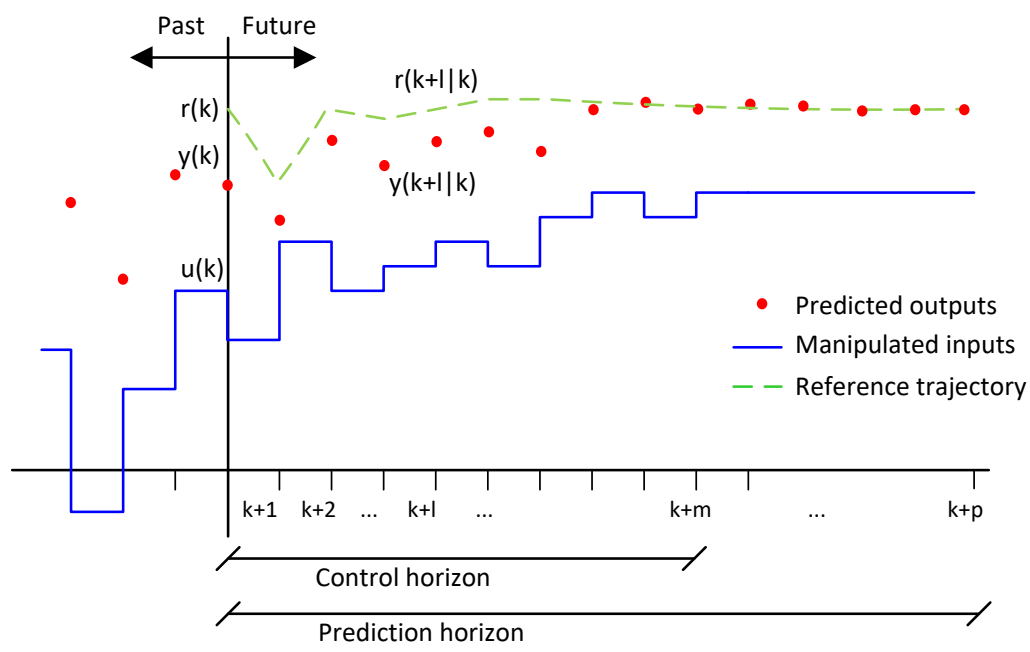

Fig. 1.2 - Model predictive control (MPC) simplified formulation. Manipulated inputs (u) along a control horizon of $m$ discrete-time steps are worked out such that, the predicted outputs ( $y)$, and 
the reference trajectory $(r)$ have a minimum difference along the prediction horizon of $p$ discretetime steps.

\subsubsection{REAL-TIME OPTIMIZATION}

Real-time optimization (RTO) has been a vigorous research field since the 1980s, almost alongside MPC, although it is not as spread in industry as MPC due to higher modeling requirements, and the need of plant steady-state operation to successfully update calculations, among other reasons. RTO may be described as a supervisory control scheme, based on steady-state process models, which is used to determine optimal setpoints of manipulated variables such that the process economy is optimized and process constraints satisfied. Darby et al. (2011) provide an insightful overview and assessment of documented RTO practices which mostly remain valid today, the analysis is primarily focused on RTO-MPC arrangements since these have been the most popular (see Fig. 1.3). A comprehensive and more recent summary of RTO current methods including an actual process case study implementation is presented by de Prada and Pitarch (2018). Another approach on process optimization uses appropriate selection of control structure along with RTO (without MPC) to achieve maximum process profitability, this has been well discussed by Engell (2007).

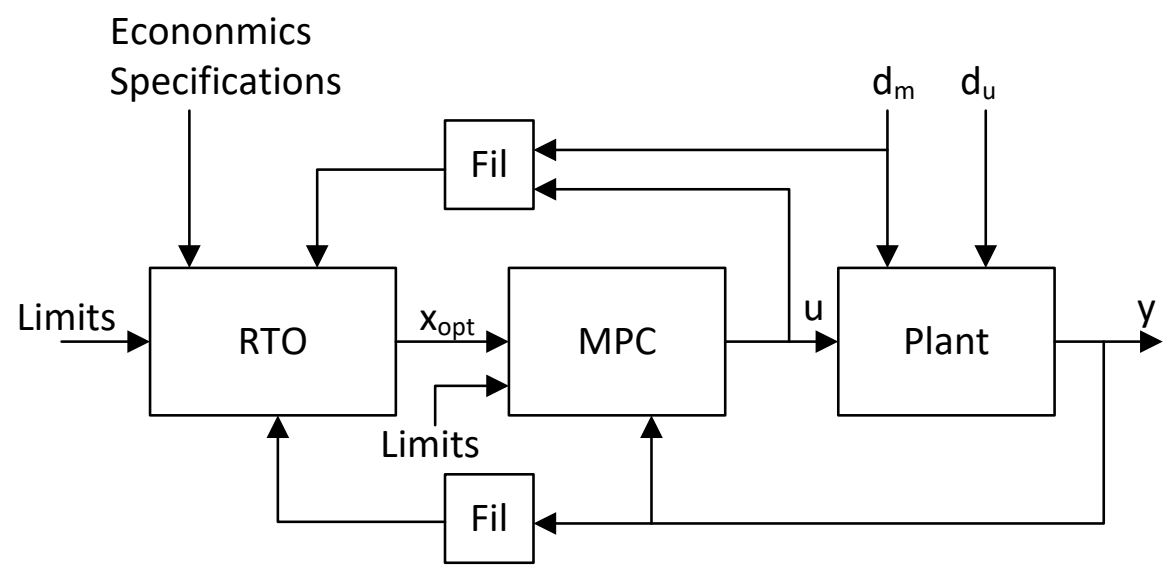

Fig. 1.3 - Simplified block diagram. Fil: filtering of signal. $d_{m}$ : measured disturbances. $d_{u}$ : unmeasured disturbances. $u$ : input variables. $y$ : output variables. $x_{o p t}$ : RTO optimal decisions that are passed on to MPC. 
Typically, RTO applications carry out multiple functions resulting in optimal setpoints (SP) to be passed on to controlled variables (CV) of MPCs underneath in the control structure (see Fig. 1.4). In brief, the first function is steady-state detection required to validate reasonable steady-state conditions of the process, this determines whether the rest of the functions are undertaken or not. If steady-state is validated, data treatment of all process data is performed, primarily gathering raw values, standard deviation figures and even essential logical functions (e.g. valve positions, flow directions in bidirectional lines) to help understand the actual condition of the process. These results feed the data reconciliation problem, aimed at reconciling process data of multiple source producing process values and parameter estimations that are consistent with the plant model. It is not until the last stage of the RTO, where the reconciled process data is used as input for running an optimization, which works out optimal set-points of process variables and gives steady-state set-points to CV in the MPCs. In addition, the RTO produces useful information such as up-to-date model parameters and operating performance figures, which are fed into upper hierarchy levels as shown in Fig. 1.4.

Currently, there is important interest on MPC and RTO integration, either as a whole dynamic RTO (Aho et al., 2009) or as an economic MPC (Amrit et al., 2013; Ellis et al., 2014; Ellis and Christofides, 2015; Engell, 2007), or separately with focus on robust and stable integration (Alvarez and Odloak, 2010; De Souza et al., 2010). Ideas of the incorporation of economic objective functions into the MPC formulation emerged in the 90s as nonlinear MPC flourished in the research community. For some early applications using economic objectives in an MPC formulation see (de Prada and Valentín, 1996; Forbes et al., 1992; Forbes and Marlin, 1994; Gonzalez Santos et al., 2001; Young et al., 2002). I would suggest read (Amrit et al., 2013; Ellis et al., 2014; Ellis and Christofides, 2015) for tutorial reviews on economic MPC and dynamic RTO, their methods and challenges. 


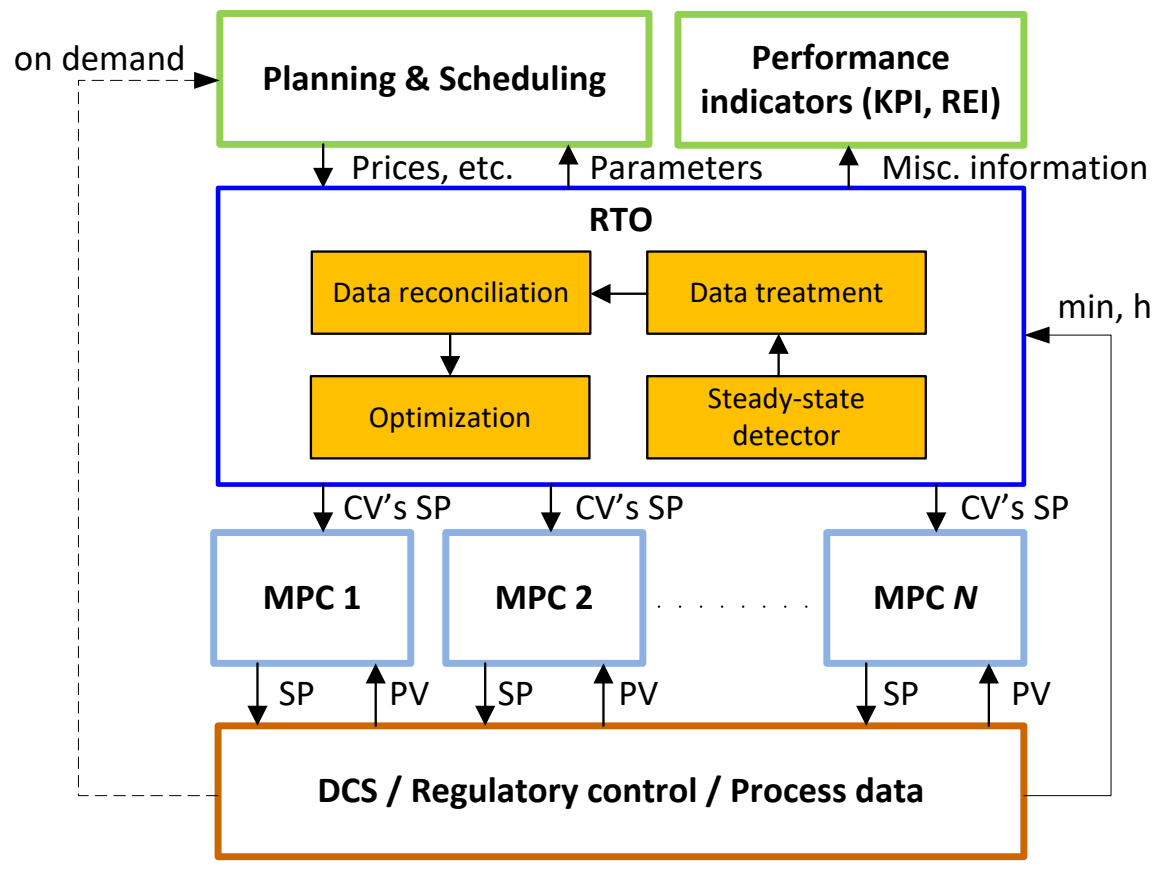

Fig. 1.4 - Typical architecture of an RTO and its information exchange with Planning and Scheduling (upper hierarchical level) and N MPC modules (lower hierarchical level), and process data updates. CV: controlled variables. SP: set-points. DCS: distributed control system. PV: process values. KPI: key performance indicator. REI: resource efficiency indicator.

Finally, open-loop strategies combined with online data reconciliation and optimization considering MPC restrictions have been deployed at industrial scale for a hydrogen network case study (de Prada et al., 2017), this work paved the way to further research on explicit account of process uncertainties by implementation of stochastic programming techniques (Galan et al., 2019b; Gutierrez et al., 2018).

\subsubsection{USE OF DECISION SUPPORT IN THE PROCESS INDUSTRY}

Even though it is widely accepted that decision support tools are instrumental in systematic decision-making in manufacturing processes, its use in process industry remains very limited. This may be partly due to lack of DSS standardization, for example, architecture guidelines and common communication protocols with client and server tools in the system (e.g. advanced control modules, equipment monitoring, basic control). In this respect, some interesting initiatives of new industrial standards are under 
development for process automation such as Open Process Automation ${ }^{\mathrm{TM}}$ Forum (OPAF, 2018) and the User Association of Automation Technology in Process Industries (NAMUR, 2018). OPAF is developing the O-PAS standard of communication which would be the key enabler of data exchange across the DSS they envision (see Fig. 1.5). Other instrumental roles in the OPAF architecture are the distributed control nodes (DCN) which exchange data with the connectivity framework (OCF), easing interoperability across the DSS. On the other hand, NAMUR proposes a mixed approach of plant-central with plant-wide monitoring and optimization in both (see Fig. 1.6). In addition, uses currently available OPC UA protocol to communicate with core process control modules (typically proprietary interfaces) and NAMUR's open standard (NOA).

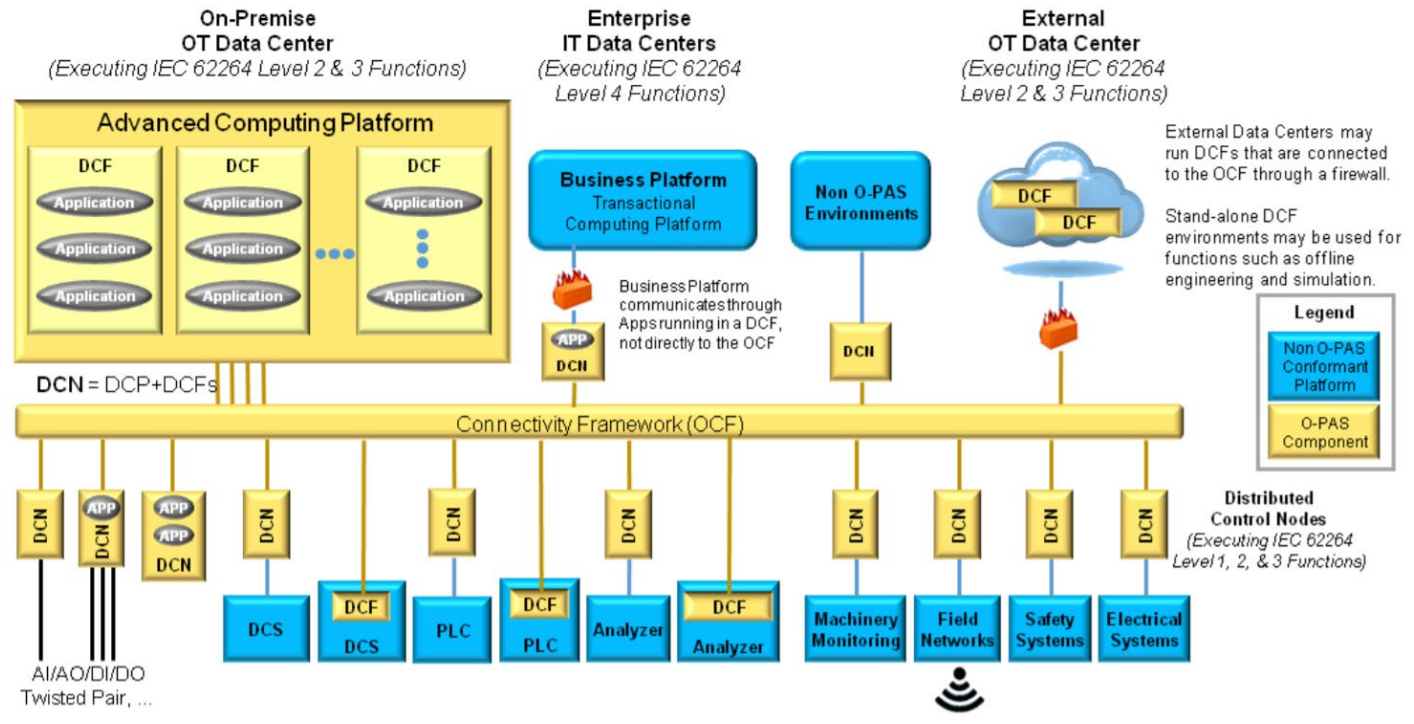

Fig. 1.5 - Vision of open system architecture by Open Process Automation Forum (OPAF, 2019). 


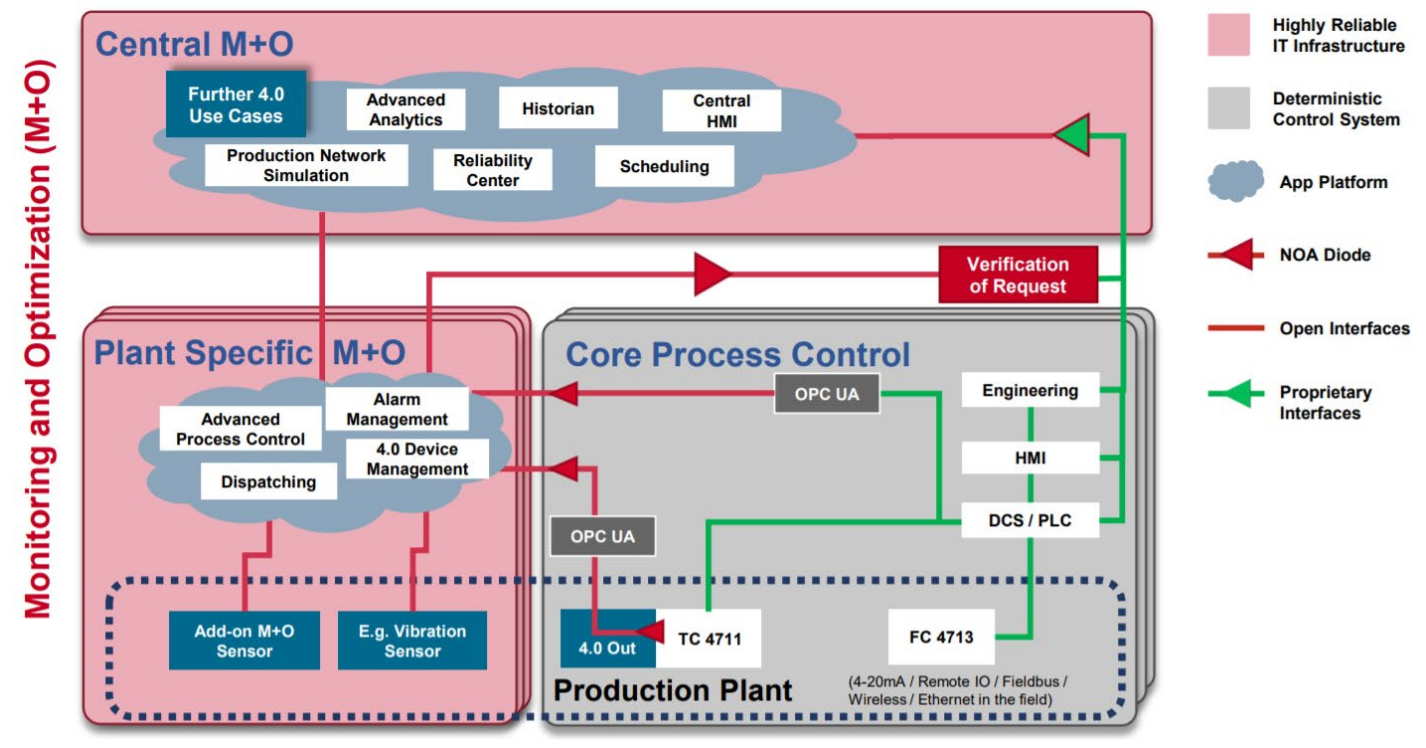

Fig. 1.6 - Overview of NAMUR open architecture (NAMUR, 2020)

Currently, decision-making is mostly conducted at individual department levels, rather than seamlessly across the company. Consequently, the integration of information is neither systematic nor automatic relying on coordination meetings. For example, planning specialists take ownership of the planning tools and communicate the plan down to the operations and scheduling specialists. Typically, coordination meetings help in aligning all incumbent teams together and in following up the plan, especially when this suffers changes. Moreover, there are limited common sources of information available other than plant data, which is effectively in detriment of open and successful discussions. Additionally, both operations and scheduling teams have their own decision support tools, which most of the time are used in a standalone manner. For example, plant control and operation is managed from control rooms using distributed control systems (DCS) with limited intervention (if any, other than for maintenance) of personnel outside operations and control. In parallel, projects of various timespans may be addressed by process engineering teams using dedicated simulation and even optimization tools, again giving critical inputs to decision makers. 
Information resides in silos managed by specialists, which blocks seamless communication of operation and even business decisions to stakeholders. Moreover, this workflow hampers efficient decision making itself, as the right information may or may not be available to decision makers in real-time or on demand. Thereby, decision support tools are actually implemented unsystematically, which leads to significant amounts of time spent on either information exchange amongst silos (most of the time manually) or chasing up specialists across business areas in search for information. Naturally, these issues may be overcome if decision support tools shared a common framework with seamless information exchange. This would enable efficient communication of decisions and effective conveyance of messages, while help in executing the right actions in a timely fashion.

To the best of my knowledge, poor processing of raw data is another key weakness of nowadays decision-making practise in most manufacturing sites. As a consequence, critical decision end up being based on data trends or simplified statistical analysis, typically in a one-through fashion, which effectively undermines any chances of arriving to optimal results. For example, personnel at control rooms take actions based on trends of key variables, mostly relying on their individual judgement and expertise. Unfortunately, under these circumstances it is unlikely that control room personnel are able to figure out the best solution to issues requiring their action, since they lack of appropriate information to do so. In the end, these contribute to the gap between best operation and actual operation results. Instead, real-time simulation or optimization tools may well support operators in their decision-making process with actual operating alternatives or actions suggestions for them to implement. Certainly, these advanced analysis tools should be accessible from control rooms in order for operators to utilize them.

Not only information exchange may be an issue, but also knowledge and skills transfer is. Senior personnel in their last years before retirement age may struggle to support more junior personnel in their knowledge and skills development, something critical at all levels in organizations. In order for senior personnel to transfer their 
knowledge efficiently the clearer the information the better. This allows less experienced staff to gain confidence and familiarize with their specific tasks, sooner rather than later, as key information resides in a common source accessible to them. Consequently, seniors can bring support along the learning curve of trainees in a timely manner, securing knowledge stays in the organization once they retire. In this regard, decision support systems (DSS) help get the right information, to the right staff, at the right moment. Additionally, systems feature extensive databases for review and revisit previous cases with their actions and consequences. It should be pointed out that this is not referring to regular databases with instrumentation and measurements data. The key point is that decision support systems archive information, rather than data. For example, past recommended actions for certain operating case would be useful for trainees to have at hand while getting up to speed in new positions.

\subsection{DECISION-MAKING UNDER UNCERTAINTY}

Decision support tools are normally based on process models that represent the plant behavior which are fed with process data. Nevertheless, the results of these models are never perfect representations of the reality, in other words subjected to uncertainty, due to multiple reasons, ranging from incorrect data (e.g. measurement errors) to structural or parametric errors of the model or process changes, amongst other causes. This may result in incorrect predictions of plant behavior, and consequently wrong decisions. Depending on the purpose of the decision support application, the problem with uncertainties may be addressed in different ways in consideration to the following characteristics:

- What is the timescale of the problem? Minutes, hours, days, weeks, months or years,

- What is the nature of the variables in consideration? Integer, continuous or mixed,

- What is the nature of the application itself? Online, on demand or offline, closed-loop or open-loop. 
In addition, uncertainties themselves may be of exogenous or endogenous nature, depending on the influence of model variables. Sources of uncertainty taken from conditions outside the scope of the model, such as the demand of a certain good to be produced in a process plant, are called exogenous. In contrast, endogenous uncertainty refers to those that depend on decision variables of the model, such as crude oil wells yield, which is only uncertain once it has been decided to drill that well. For the purpose of this thesis, uncertainties explicitly considered are exogenous. See (Goel and Grossmann, 2004; Grossmann et al., 2016; Gupta and Grossmann, 2011) for more detailed discussion on exogenous and endogenous uncertainty and applications on case studies.

Having in mind the answers to those questions, I want to highlight the most relevant strategies for incorporating uncertainty in the decision-making process, namely:

- data reconciliation and parameter estimation,

- modifier adaptation,

- $\quad$ simulation-based methods,

- optimization under uncertainty techniques

For the sake of contextualization I will refer to these four approaches which are the most relevant to this thesis, acknowledging that other approaches might be feasible as well. In the following paragraphs I briefly review these alternatives.

\subsubsection{DATA RECONCILIATION AND PARAMETER ESTIMATION}

Apart from the natural process deviations (a.k.a. noise), it is likely to face measurement inconsistencies across plant data sources. For example, redundancy of plant and laboratory measurements can prove to be very effective in improving accuracy, although these may fail in providing an accurate value. In this respect, one straightforward alternative is to adjust model variables and parameters so that it matches real data, namely apply data reconciliation and parameter estimation. For this reason, reconciliation of plant data against a given model has become of key importance at the time of delivering the best estimates available. In addition to model reconciled figures, 
unmeasured variables may be available as well, provided those are considered in the model.

In brief, data reconciliation (DR) solves an optimization problem that corrects actual measurements, while simultaneously estimates the value of model parameters such that the corrected measurements satisfy the model and are as close as possible to the actual measurements. In order to achieve this objective, DR requires a suitable model of the system on which to compare plant data, and enough measurements to work out a meaningful reconciled outcome. On top of these, computation times must be considered, especially if the calculation is meant to be online. Sarabia et al. (2012) presented a successful industrial pilot application of online DR in an oil refinery hydrogen network, later extended by de Prada et al (2017) for the integration with a real-time optimization (RTO), and Galan et al. (2018) who discusses the validation process of the pilot tool.

One main stream category implies techniques considering uncertainties in plant model parameters, which typically rely on some data source that should drive parameter estimations such that model values and measurement minimize their difference. This approach is considered in this study and further explained in the next sections.

\subsubsection{MODIFIER ADAPTATION}

Another relevant category works out the uncertainties by considering model structural errors, which are corrected iteratively using plant data and other methodspecific coefficients modifiers. Basically, plant-model mismatch is solved incorporating gradients from process measurements, which are used to compute iteratively modifiers and actual process optimal. In the end, rather than changing the model to satisfy actual data, it is changed the optimization problem itself so that the modified problem matches actual data. This approach, is essentially based on integrated system optimization and parameter estimation (ISOPE) methods introduced by Roberts (1979), which presented an algorithm capable of reaching plant optimum regardless of structural model mismatch with the actual plant. This was further refined by numerous researchers, such as Forbes 
and Marlin (1994) and Tatjewski and Tadej (2001) to name some. Eventually these works derived in what nowadays is known as adaptation methods, Gao and Engell (2005) being among those who first coined the term. Later Chachuat et al. (2009) presented a thorough description and categorization of all adaptive methods, while favoring modifier adaptation over the rest. Rodríguez-Blanco (2017) provides an insightful state-of-the-art review and presents an industrial case study. Modifier adaptation is a widespread and well known method in real-time optimization research community.

\subsubsection{SiMULATION-BASED METHODS}

Simulation-based decision-making applications heavily rely on their forecasting capability, which is used to anticipate system behavior and how better implement mitigation measures if needed. Thereby, supporting the assessment of scenarios, those can be random or pre-set to study a particular condition of the process. For example, hazardous operations studies (HAZOPs) and What-if analysis (WiA). In chapter 3 of this thesis a brief What-if analysis example is presented within the case study.

In addition, simulations require data inputs, which may or may not be accurate for a given process due to measurement errors. Therefore, feeding models with reconciled data is critical in online and offline simulation to properly represent process behavior. In this regard, either the simulation environment is capable of sorting data errors or data inputs are sanitized externally prior these are fed into the model for simulation execution. Typically, the latter is preferred if an external reconciliation tool is already available, while in general, data errors treatment is managed within the simulation environment. However this is done, data treatment should provide sufficiently good inputs for a simulation to forecast at its expected level of accuracy, which should be enough to make sensible decisions. In this thesis, I leverage an already implemented data reconciliation tool in the Petronor refinery (Sarabia et al., 2012) for feeding treated and reconciled data into simulation models. This point is elaborated further in chapter 3 and chapter 4 .

Refer to Appendix B for introductory notes on simulation-based decision support. 


\subsubsection{OPTIMIZATION UNDER UNCERTAINTY}

On the other hand, optimization-based approaches rely on different techniques of optimization under uncertainty such as, stochastic programming and fuzzy programming to mention some of them. The main differentiator with deterministic optimization is that uncertainty is explicitly considered in the model either in variables or parameters. These have been a very fruitful research ground, however still with quite limited industrial application, probably, due to its complex formulation and typically higher computation demand compared to simulation-based methods or deterministic optimization. Grossmann et al. (2016) presented a tutorial review on recent advances in the field of optimization under uncertainty with focus on process systems problems. I revisit a stateof-the-art review article by Sahinidis (2004), which is mostly valid to this day, to provide a succinct overview of each methodology for the sake of contextualization. In general only highlights of methods are mentioned hereinafter to provide a broad background of the topic. More detailed descriptions follow for the optimization techniques implemented in their respective chapters.

\subsubsection{STOCHASTIC PROGRAMMING}

Stochastic programming essentially consists of modeling and optimization of mathematical models that include a combination of random variables and parameters (in the rest of the section, the term variables refers to variables and parameters for simplicity). These random variables may affect differently each model depending on, when in the decision time window uncertainty realizes, and whether or not constraints are considered with certain probability of occurrence. These are addressed by multi-stage stochastic programming and probabilistic programming depending on the nature of the problem. Refer to Birge and Louveaux (2011), widely accepted as the flagship textbook in the field, for a comprehensive introduction into stochastic programming. 


\subsection{MULTI-STAGE STOCHASTIC PROGRAMMING}

The simplest case is the two-stage stochastic problem, meaning that decision events are grouped in two sets namely first stage and second stage. For simplicity I refer to two-stage stochastic programming, however the concepts commented herein are all applicable with the appropriate function changes. First-stage variables are those decided before actual realization of the uncertainty (so called here-and-now decisions). Secondstage variables are those decisions actioned once uncertainty is revealed. Therefore these are used as recourse actions to accommodate infeasibilities or higher operating costs due to first-stage decisions. In this context, the second-stage variables are random variables, along with their cost function. The most popular formulation considers an objective function made up of the sum of the cost function with first-stage variables and the expected value of the second-stage random variables (Birge and Louveaux, 2011). The recourse framework of two-stage stochastic programming has been applied to linear, integer, mixed-integer linear, nonlinear and mixed-integer nonlinear programming. For example, the general form of two-stage stochastic programming problem is as $(1.1,1.2)$.

$$
\min _{u_{F}, \xi} J_{F}\left(u_{F}\right)+\mathbb{E}\left\{Q\left(u_{F}, \xi\right)\right\} \quad \text {, s.t. } g\left(u_{F}\right) \leq 0, u_{F} \in \mathbb{U}_{F} \subseteq \mathbb{R}^{n 1}, \xi \in \Xi \subseteq \mathbb{R}^{k}
$$

with

$$
Q\left(u_{F}, \xi\right)=\min _{u_{F}, u_{S}, \xi} J_{S}\left(\xi, u_{F}, u_{S}\right), \text { s.t. } G\left(\xi, u_{F}, u_{S}\right) \leq 0, u_{S} \in \mathbb{U}_{S} \subseteq \mathbb{R}^{n 2}
$$

Where $\xi$ is a random variable, $u$ are decision variables with subscripts denoting whether they belong to the first-stage $\left({ }_{F}\right)$ variables set or second-stage variables $(\cdot s)$ set. Similarly, $J$ is the cost function with its subscript denoting first or second stage domain. $\mathbb{E}$ denotes the expected value of the cost function $(Q(\cdot))$, a.k.a. recourse function for it accounts realizations of all uncertain events, or scenarios in the case of considering discrete probability distribution functions of $\xi$. Finally, $g(\cdot)$ and $G(\cdot)$ represent the model and its constraints within first-stage and second-stage domains respectively. This formulation implies the decision-maker is risk-neutral as there is no accountability of risk whatsoever. 
Additional refinement can be incorporated in the objective function to reflect risk awareness, so called risk-averse formulation a.k.a. robust stochastic programming (Sahinidis, 2004) (1.3).

$$
\min _{u_{F}, u_{S}(\cdot), \xi} J_{F}\left(u_{F}\right)+\mathbb{E}\left[Q\left(u_{F}, \xi\right)\right]+\lambda \cdot f\left(\xi, u_{S}\right), \text { s.t. } \lambda \geq 0
$$

Where $f$ is a variability measure, e.g. variance, of the second-stage costs and $\lambda$ a positive scalar that measures the risk level desired. Thereby, large values of $\lambda$ imply low variance and small $\lambda$ reduce expected costs (Mulvey et al., 1995; Sahinidis, 2004).

In chapter five of this thesis I review two-stage stochastic programming further as it is implemented to address risk-neutral and risk-averse uncertainty in one case study.

\subsection{PROBABILISTIC PROGRAMMING}

The recourse approach enables delaying decisions further in order to accommodate the objective function costs linked to the second-stage variables. Probabilistic programming, a.k.a. chance-constraint programming, set probability levels to constraints in the second-stage. In other words, instead of imposing feasibility it allows for certain infeasibility to occur provided it is within a given probability. That is why it is likely to achieve better cost results than multi-stage stochastic programming, since its constraints are somehow relaxed.

\subsubsection{FUZZY PROGRAMMING}

Fuzzy programming models uncertainty considering random parameter as fuzzy members and constraints as fuzzy sets. The so called membership function of the constraint defines the level of satisfaction of constraints, therefore enabling constraint violations within this preset range. Objective function in fuzzy programming is bounded, treated as a constraint with upper and lower bounds determining the decision maker's expectations (Sahinidis, 2004). Refer to Zimmermann (2001) for a thorough introduction to theory and applications of fuzzy programming. 


\subsection{HYDROGEN NETWORKS OF CRUDE OIL REFINERIES}

Crude oil production and refining remains a critical energy source for world economies, although it is expected to reach its peak around 2030 to 2040. The main reason for this is the industrial growth forecasted in emerging economies as India and countries in Africa (IEA, 2019). However the reason, it is widely accepted that crude oil refineries will face steadily increasing pressure for carbon dioxide $\left(\mathrm{CO}_{2}\right)$ emissions reduction (UNFCCC, 2015), while required to produce market demanded fuels compliant with tighter environmental legislation including sulfur content reduction among other parameters. Therefore, sulfur removal processes in the crude oil refining industry plays a paramount role that it is expected to rise in the foreseeable future.

Given this background, hydrogen is of key importance in modern crude oil refining as the main resource consumed in hydrodesulfurization and hydrocracking technologies. The core purpose of hydrodesulfurization is sulfur removal from intermediate hydrocarbon streams prior to blending these into final fuel products such as gasoline and diesel. Hydrocracking, on the other hand, is aimed at breaking down long chain hydrocarbon molecules producing lighter components with higher market value, while also carrying out hydrodesulfurization. In addition, hydrogen is required in multiple conversion-focused process units, essentially, used in olefin and polyolefin saturation at pre-treatment or post-treatment stages. I focus the following overview on hydrodesulfurization for it is by far the most relevant hydrogen consumption technology in refineries. Among the principal characteristics affecting hydrogen consumption in hydrodesulfurization are the following:

- sulfur load of feedstock, the higher the sulfur content the higher the hydrogen consumed,

- sulfur species composition of feedstock, mercaptans (R-S-H) requiring the least severe conditions of treatment and dibenzothiophene (two benzene rings bound by thiophene in the middle, B-T-B) demanding the most severe, i.e. higher hydrogen pressure and reaction temperature (Froment, 2004; Ho, 2004), 
- feedstock cuts, the lighter the cut the less hydrogen demand being gasoline cuts the least demanding and vacuum residue the highest demanding cuts,

- catalyst selectivity over side reactions (Kouyionas, 1990), hydrogen is consumed in side reactions such as hydrodenitrogenation (nitrogen removal), hydrodeoxigenation (oxygen removal), hydrocracking, catalyst sulfiding, among others,

This results in very specific process conditions within individual process units performing hydrodesulfurization across assets in a refinery. In order to cope with demand and process requirements, consumers are supplied with different hydrogen purities through pipeline networks connected to battery limits of process units. These hydrogen networks have:

- $\quad$ sources of high purity (HP) hydrogen, typically steam reformers producing $99 \%$ to 99.9\% pure fresh hydrogen from methane and steam, or purification units providing HP hydrogen in the range of 90-99.9\% from low purity sources.

- $\quad$ sources of low purity (LP) hydrogen, typically of two origins and purity in the range of $70 \%$ to $85 \%$, hydrogen rich streams from recycle or off gases of hydrodesulfurization sections in process units, hydrogen produced as by-product in naphtha octane improvement processes in reforming units producing reformate (component of gasoline blending) as main product,

- sinks of hydrogen (LP and HP), represented by all hydrogen consumer process units regardless of the demand and purity.

- off-gas routed to fuel gas header, lean hydrogen outlet streams of gas-liquid separation sections of process units are routed to the refinery fuel gas header and consumed as fuel in fired heaters.

It is critical to understand the fundamentals of individual process units to further comprehend their impact at all levels across the refinery network. In fact, due to process units being heavily interconnected, hydrogen network management actually impacts individual and global yields of plants across the refinery. Therefore, it is important to define exactly the scope of work when addressing hydrogen networks, as well as a basic 
grasp of the process network outside the boundaries of the hydrogen network to enable more realistic analysis of any system.

\subsubsection{HYDRODESULFURIZATION UNIT DESCRIPTION}

In the following lines I describe the basic sections of a hydrodesulfurization unit (see Fig.1.7), including mentions to key pieces of equipment, and state which sections are most relevant to hydrogen network management.

Hydrodesulfurization process units can be broken down into the following sections (Fig. 1.7):

- Feed preheating,

- Furnace,

- Reaction,

- High pressure separation,

- Recycle and Make-up gas,

- Middle pressure separation,

- Low pressure separation,

- Product stripping,

- Product cooling. 


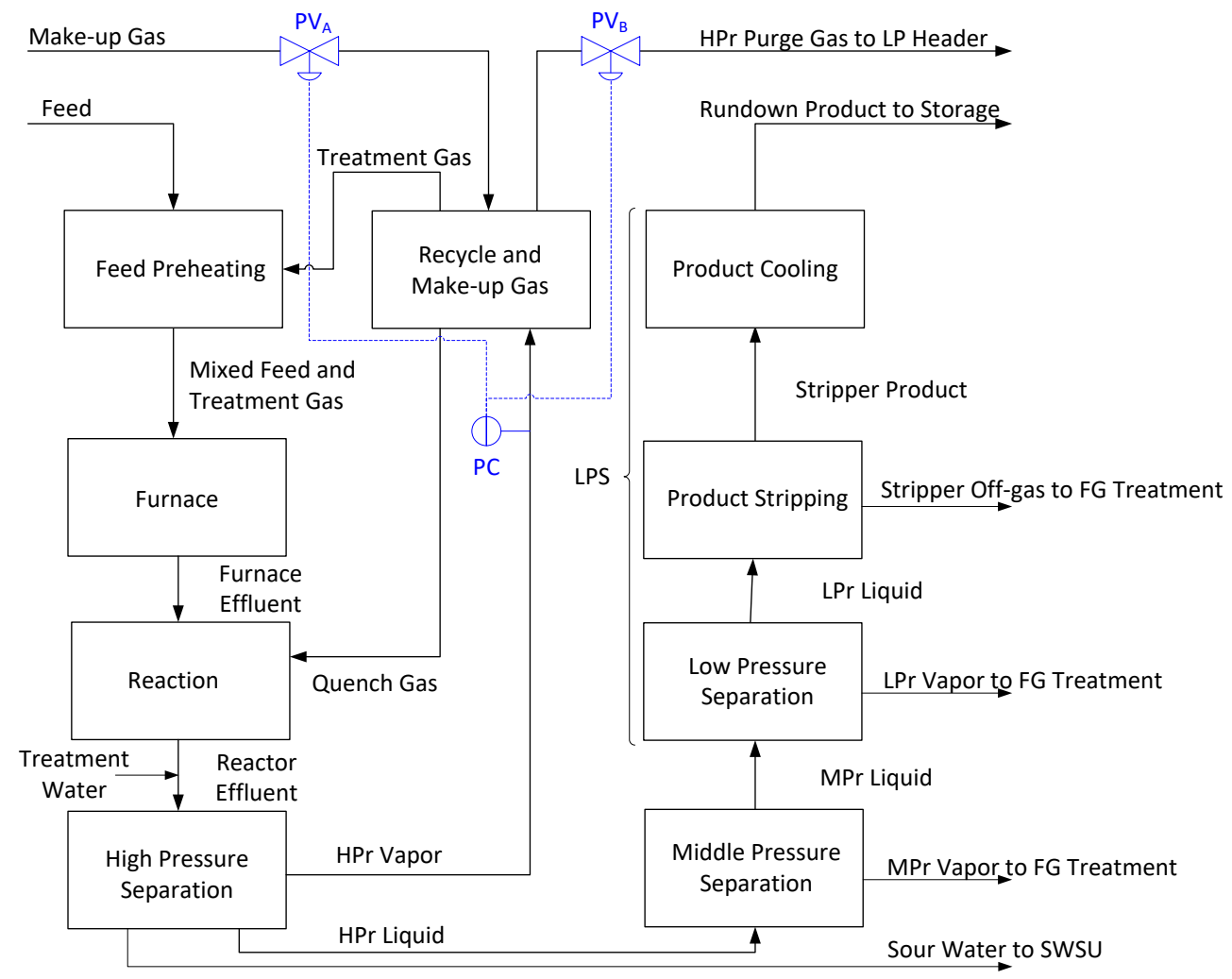

Fig. 1.7 - Simplified hydrodesulfurization process block diagram. LPr: Low pressure; MPr: Middle pressure; HPr: high pressure; LP: Low purity; LPS: low pressure separator, including product stripping and product cooling; $\mathrm{PC}$ : pressure controller; $\mathrm{PV}_{\mathrm{A}}$ : make-up gas pressure valve; $\mathrm{PV}$ : $\mathrm{HPr}$ purge gas pressure valve; SWSU: sour water stripping unit.

In the feed preheating section, feeds from various sources are received in a surge drum and pumped to the reactor through a number of heat exchangers where feed is preheated against reactor effluent. Additionally, in this section the feed is mixed with treatment gas from the recycle gas and make-up section.

The mixed feed and gas stream is heated in the furnace heating section through a fired heater designed to increase temperature of the process stream to reach the required reactor inlet temperature.

The furnace effluent enters the reaction section where the fixed bed catalytic reactor is the main piece of equipment. The reactor is designed to remove sulfur from the 
feed through hydrodesulfurization consuming hydrogen at high temperature, producing low sulfur hydrocarbons and by-products as hydrogen sulfide $\left(\mathrm{H}_{2} \mathrm{~S}\right)$, ammonia $\left(\mathrm{NH}_{3}\right)$ and light gases (e.g. methane, ethane). The exothermic chemical process occurs in fixed catalyst beds inside the reactor vessel increasing temperature as a consequence of chemical conversion from inlet to outlet streams. Between catalyst beds a quench gas stream from the recycle gas circuit is injected to keep temperature controlled along the reactor beds. Reaction equilibrium is shifted toward products by stoichiometric excess of hydrogen, which, in addition is required for preventing premature catalyst decay. The reactor effluent is cooled by exchanging heat with feed and treatment gas in heat exchangers, and routed to the high pressure (HPr) separator. Treatment water is injected in the reactor effluent to dissolve sulfide-ammonia salts that promote corrosion and plugging in heat exchangers as the effluent is cooled. The HPr separator is a three phase separator that is designed to enable entrained gases to separate (vapor phase) from the oil and water liquid phases. The vapor phase is hydrogen rich with some impurities, mostly light gases and $\mathrm{H}_{2} \mathrm{~S}$. The oil phase is routed to the middle pressure (MPr) separation section via a flashing valve. Sour water is sent to a sour water stripping unit for further treatment and reutilization.

Vapor from the HPr separator is sent to the recycle compressor through an amine absorber to remove $\mathrm{H}_{2} \mathrm{~S}$. Recycle gas exiting the recycle compressor is routed to the preheating section and to the reaction section as quench gas. Fresh hydrogen rich makeup gas is compressed in the make-up compressor and is mixed with recycle gas into treatment gas, which is the actual stream preheated and mixed with the feed. The pressure control of this section may be either controlling the HPr purge stream or controlling the make-up stream (see the control loops in blue in Fig. 1.7). The former leaves the make-up as a degree of freedom (manual operation of $\mathrm{PV}_{\mathrm{A}}$ ) since for any feasible make-up flow the HPr purge is adjusted to keep the pressure under control $\left(P V_{B}\right.$ is controlled by the PC in closed-loop). The latter leaves the HPr purge gas as a degree of freedom (manual operation of $\mathrm{PV}_{\mathrm{B}}$ ) since for any feasible HPr purge gas the pressure control adjusts the make-up ( $\mathrm{PV}_{\mathrm{A}}$ is controlled by the $\mathrm{PC}$ in closed-loop). This is the most 
efficient control in high purity units, as it allows an operation with closed or almost closed HPr purge depending of the specific application.

The MPr separation and the low pressure (LPr) separation sections comprise liquidgas separators at different pressure levels. Essentially, at each pressure level a specific amount of dissolved gases remain in the oil phase (depending on temperature and composition of the gas species), and the settling conditions in the separators promote those to be released in the back end. The vapor stream of both separators is composed by light gases and some $\mathrm{H}_{2} \mathrm{~S}$, and therefore routed to the fuel gas treatment unit for $\mathrm{H}_{2} \mathrm{~S}$ removal prior to being sent to the fuel gas header for general consumption in furnaces.

The oil stream from the LPr separator enters a product stripper designed to remove any light gases and $\mathrm{H}_{2} \mathrm{~S}$ left in the liquid by steam stripping so that it meets product specifications and is free of dissolved gases. The off-gas from the stripper is sent to the fuel gas treatment unit for $\mathrm{H}_{2} \mathrm{~S}$ removal. The stripper product is routed to the product cooling section and finally as rundown product to storage. Notice that from a modeling standpoint the LPr separation might well include all process sections from LPr separation to product cooling. Therefore, the product of the "new" LPr separation is free of gases ready to be sent to storage. In this approach, LPr vapor would include all gases dissolved in the MPr liquid. I revisit and develop this point across the thesis in relevant sections.

A schematic of a real middle distillates hydrodesulfurization unit that produces low sulfur diesel is shown in Fig. 1.8 to provide a clear contrast with the simplified block diagram in Fig. 1.7. A more detailed description of the process is out of scope of this thesis. 


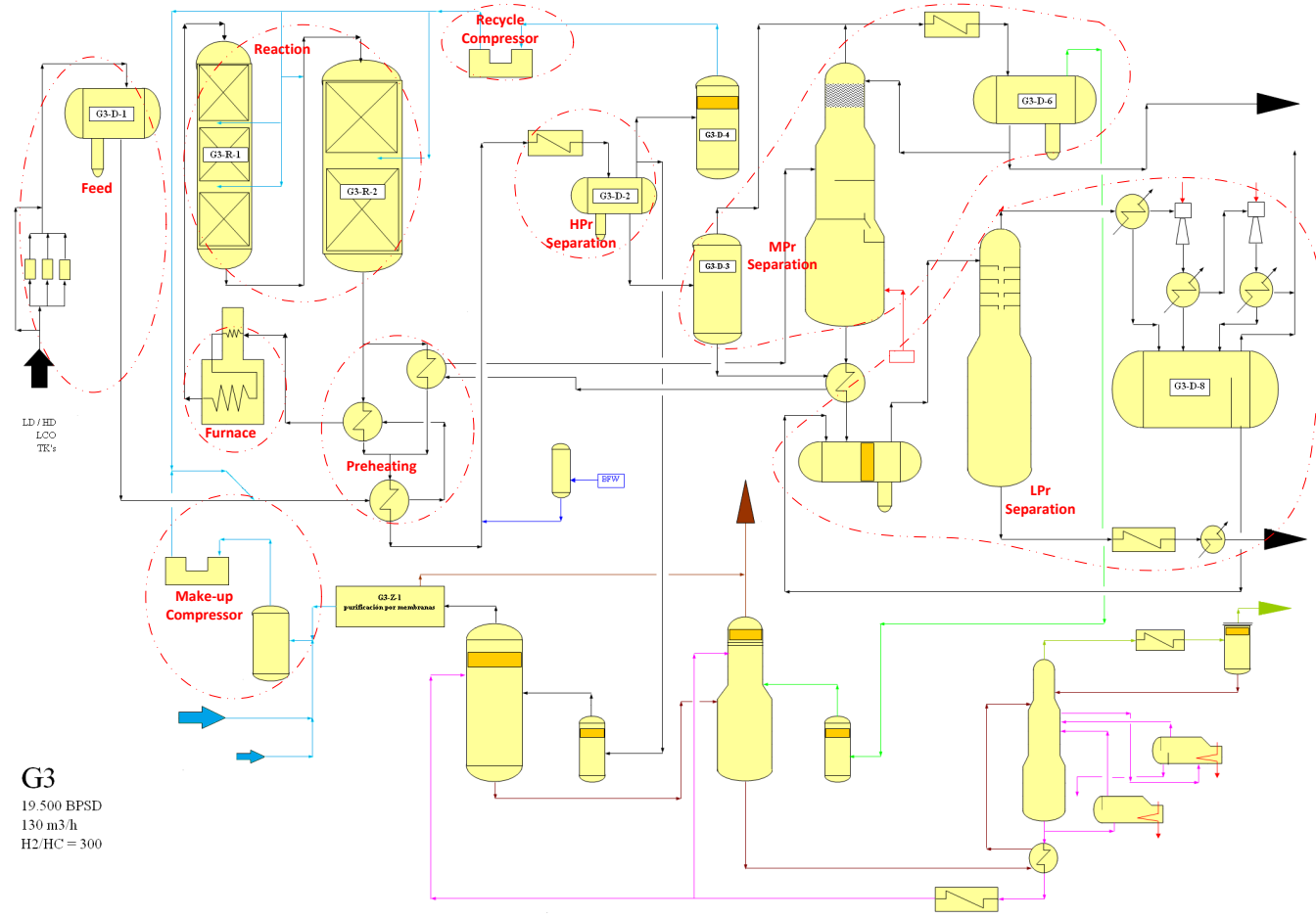

Fig. 1.8 - Schematic of a real middle distillates hydrodesulfurization unit with different sections circled in red. Source: Petronor ( n.d.).

\subsubsection{CHARACTERISTICS OF HYDROGEN NETWORKS}

Even though industrial setups of hydrogen networks are variable, there are key common characteristics across most of them. Hereinafter, I briefly highlight the most remarkable common aspects of hydrogen networks in real refineries for the sake of contextualization of the case study. This is expanded in chapter 3 and chapter 4.

Firstly, networks comprise a large proportion of process units within the refining complex, since hydrogen consuming processes are encountered in many sections of process units as well as in those focused in hydrodesulfurization as their main purpose. Several processes require hydrogen treatment (a.k.a. hydrotreatment) of feeds prior to routing streams to their main function sections (e.g. naphtha hydrotreatment section before it is sent to the reformation section in the reforming unit). Likewise, other processes demand hydrogen at their back end after their main functions are realized (e.g. 
coker naphtha hydrotreatment). Essentially these post and pretreatments are aimed at olefins and aromatics saturation as well as sulfur removal.

Secondly, hydrogen networks are heavily integrated across process units, especially with respect to low purity (LP) headers that function as sumps of hydrogen rich streams (see Fig. 1.9). Therefore, upsets in any one unit sending to the LP headers impact process conditions across the network rather than only that individual unit.

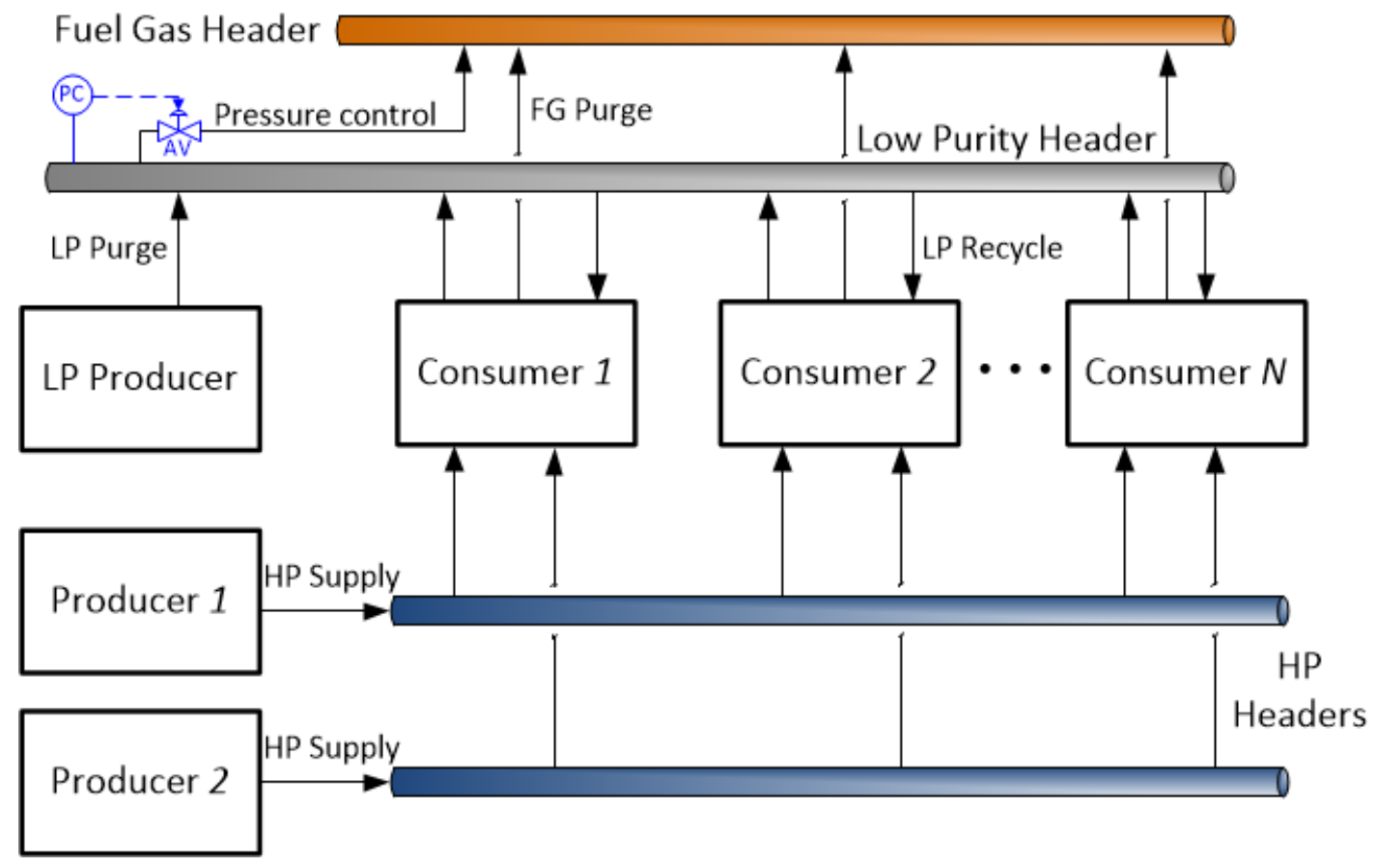

Fig. 1.9 - Schematic showing the different types of headers found in hydrogen network: high purity headers (blue), low purity header (grey), fuel gas header (brown). An automatic valve (AV) controls the pressure of the low purity header (LPH) by controlling the flow from the LPH to the fuel gas header (FGH). PC: pressure controller. AV: automatic valve.

Thirdly, the topology of actual networks is complex since these comprise: multiple process units, multiple purification units, multiple purity levels, multiple pressure levels, subnetworks with bidirectional flow, to mention the most relevant points. 


\subsubsection{OVERVIEW OF HYDROGEN NETWORKS MANAGEMENT}

Hydrogen network management has been researched for the last twenty five years (Elsherif et al., 2015), essentially driven by the increasing need of crude oil refineries and petrochemical sites for improved hydrogen network management directions. However, most of the research effort has been put on design problems, rather than real-time operation solutions. Actually, to the best of my knowledge there are few documented references to actual refinery hydrogen networks. Needless to say, references to decision support tools for refinery hydrogen networks. Therefore, hereinafter I provide an introductory overview of hydrogen networks management which considers design and operation case studies for the sake of acknowledging the state-of-art in the broader field. Nonetheless, only operating case studies are directly relevant to the scope of work this thesis.

In the mid-90s, pinch analysis was used by Towler et al. (1996) to formulate what it is considered the seminal work of hydrogen networks management, providing a graphical framework for practitioners to tackle hydrogen network design problems in a simplified manner. Other researchers followed and proposed refined methods based on the same approach (Alves and Towler, 2002; Elsherif et al., 2015; Shariati et al., 2013). Another predominant approach towards network management and design is through mathematical programming, which fundamentally leads to a MINLP problem unless simplifications are implemented, for examples refer to (Girardin et al., 2006; Jia and Zhang, 2011; Jiao et al., 2012a; Kumar et al., 2010; Lou et al., 2014; Yang and Feng, 2019). I focus the attention on the mathematical programming approach as it is the most relevant to mathematical models used in decision-making.

\subsubsection{OVERVIEW ON MATHEMATICAL PROGRAMMING IN HYDROGEN NETWORKS}

In terms of mathematical programming, Sardashti Birjandi et al. (2014) propose a global optimization algorithm capable of undertaking the design problem with promising results with respect to cost savings. Unfortunately, they do not present clear figures on 
the optimality gaps of their results or computation times which are instrumental in assessing the likelihood of implementation in actual refineries.

Yang and Feng (2019) address the design problem accounting hydrogen sulfide $\left(\mathrm{H}_{2} \mathrm{~S}\right)$ removal sections of hydrodesulfurization units, they demonstrate that this approach leads to interesting results. Basically, different combinations of $\mathrm{H}_{2} \mathrm{~S}$ removal columns give additional room for optimization, where sharing of columns proves beneficial in some cases considered. On the downside, this approach optimizes over given network setups such as quantity of $\mathrm{H}_{2} \mathrm{~S}$ columns, compressors, requiring the user to actually provide different process flowsheets beforehand whose results are later compared against in order to conclude which is the best design. In addition, they do not account for actual $\mathrm{H}_{2} \mathrm{~S}$ load according to feed flowrate, which for most process units is not negligible being actually an impurity in large proportion in separators.

Jia and Zhang (2011) present a technique for considering multi-component gas streams rather than the most commonly assumed binary gas composition. This method combines pinch analysis to give a starting point for a base case and executes sequential optimization in a four step fashion. Although this method provides a more accurate composition of gas streams impacting on more realistic figures, it has been applied on very simplified hydrogen networks (e.g. 25 total equations, 19 variables) with fair computation times. Likewise, Umana et al. (2014) propose a multicomponent hydrogen network management problem integrating detailed hydroprocessing kinetic models. The main advantage is that with kinetic reaction models, their problem is sensitive to temperature changes, impurities composition as well as sulfur content in the feed. The authors demonstrate their approach on a set of case studies with 38 variables and 70 equations. Considering that actual hydrogen network variables range around two orders of magnitude over either of these results, it is still unclear whether the these methods might be successfully performed in real world hydrogen networks.

Additionally, consideration of uncertainties in operation has been addressed by some authors in various shapes and forms. Lou et al. (2014), introduces a robust 
optimization formulation for hydrogen network design and compares it against stochastic programming formulation. Although stochastic programming shows better results in terms of total annual cost, robust optimization provides scenario insensitive results along with explicit representation of the desired risk by the decision maker. On the other hand, Jiao et al. (2012b) show a chance constraint hydrogen network design formulation and present a case study of a refinery in China. A simplified model of the network is used focusing on comparing the suitability of the method against deterministic formulation provided that some constraint violation is allowed. The authors conclude that their proposed method reduces total annual costs of the network for the data sets studied. Likewise, MINLP formulations accounting operation uncertainties at design stage (Chen et al., 2020) demonstrated how global optimization can be implemented, though it lacks of computation efficiency at the level required for industrial usability.

Process network optimal operation is another area of interest within refinery hydrogen networks that has got attention from researchers. Kumar et al. (2010) show that either MINLP or NLP models present savings over LP models when dealing with the optimal operation of hydrogen networks. The authors attribute this to a better representation of process constraints such as recycle gas purity. Jiao et al. (2012a) present an improvement to the previous approach, essentially converting the MINLP problem into a MILP and compare their results against published MINLP methods implemented by other authors.

Sarabia et al. (2012) approach the hydrogen network management problem as a two-step optimization problem, the first step performs a robust data reconciliation in realtime to adjust model parameters to actual conditions and the second step is a hydrogen distribution optimization. The main advantages of this strategy are the ease of integration with supervisory level control as well as upper levels such as planning applications, and its ability of providing "good quality" parameter estimations useful across the refinery, not only for hydrogen network management purposes. Another important aspect of this work is that addresses an actual refinery hydrogen network case study, including supervisory 
level control structures. Gómez Sayalero (2016) presents the same case study, however her focus is on how better model the different equipment along with optimal network operation in steady-state. These two works have been seminal precedents of this thesis, due to their integrated architecture into already operating advanced control tools set and extensive use of online data. These ideas have strongly shaped this work.

\subsubsection{OPEN ISSUES FOR CONTRIBUTION}

Even though, there are many studies on hydrogen network management there are challenges going forward as well that should be addressed. Hereinafter, I point out the most relevant open issues, to the best of my knowledge.

\subsection{DECISION SUPPORT INTEGRATION INTO CONTROL SYSTEM ARCHITECTURES}

The integration of optimization-based and simulation-based techniques into the control architectures applied to refinery hydrogen networks has received little attention, especially incorporating these in control rooms. Documented applications that interact with control systems (e.g. RTO, D-RTO, real-time simulation) implemented in actual hydrogen networks are scarce, to say the least. However, the economic burden of suboptimized operations is well understood and should be enough motivation for moving forward into this direction. The hydrogen network management problem has been studied from various viewpoints, nonetheless, most of the analysis has focused on the design with limited operation and control implementations addressed.

\subsection{COMBINATION OF CLOSED-LOOP AND OPEN-LOOP DECISION SUPPORT TOOLS}

Currently, most studies propose optimization solutions either in closed-loop or open-loop manner, while combinations of both have scarcely been addressed within a decision support framework. Single applications may be effective under a range of conditions, while implementing a cluster of applications closely integrated into a common framework may boost additional decision-making performance. This can certainly be linked with the digital twin concept and enterprise-wide decision support, nonetheless, 
literature has barely discussed this link something surprising given the popularity of both topics in times of the so called fourth industrial revolution.

\subsection{SYSTEMATIC ACCOUNTABILITY OF UNCERTAINTY IN THE DECISION-MAKING PROCESS}

Any model-based approach should consider uncertainties of various sources, explicitly or implicitly, in order to be implementable in real-time operation problems. In this respect most hydrogen network management documented cases accounting for explicit uncertainties address the optimal design problem, leaving real-time operation and control decisions aside. However, it is widely accepted that uncertainties faced in daily operations require models to specifically consider these, which has been researched extensively in general with limited real case studies documented. Advantages and disadvantages of different approaches to optimization under uncertainty at network level and plant level are not fully understood yet, although some studies already brought the topic on the table.

In overall, as a consequence to these gaps, I believe that a holistic standpoint would enable capturing the most useful optimization and simulation techniques leveraged in a decision support system to aid hydrogen network operation decision-making process, considering uncertain conditions.

\subsection{CASE STUDY MOTIVATION}

I use Petronor refinery hydrogen network as the central case study in this thesis. Petronor refinery is located in Muskiz, Basque Country region in Spain (Petronor, 2020), the refinery crude oil processing capacity is around $1458 \mathrm{~m}^{3} / \mathrm{h}(220000$ barrels per day). The refinery hydrogen network comprises 14 consumer units and four producer units, with several units exhausting HPr purge gases into a LP header and two purification units within individual units' battery limits. For the sake conciseness I describe further details on the control structure and hydrogen network management setup in the relevant sections of the thesis. 
Due to the complexity and broad variety of refinery hydrogen networks it is worthwhile focusing on a specific case study as the main driver of the discussion throughout this thesis. However, to the best of my knowledge, the issues addressed are general to refinery hydrogen networks to some extent.

\subsubsection{SCOPE OF WORK}

This work assumes that successful process operation decision-making can be enhanced by the right set of decision support tools, alongside suitable information of the process. In this regard, this thesis is scoped in the field of control and operation of processes with especial attention to the decision-making process of decision makers. The scope of work and hypothesis under which this thesis is structured, considers the open issues and potential contribution areas, described in section 1.3.3.2, with respect to simulation and optimization techniques used as decision support tools in real-time operation of refinery hydrogen networks.

In summary, this thesis focuses on the following open issues in the field of refinery hydrogen networks and its associated decision support systems:

- Scarce integration of decision support tools with real-time systems and control systems architectures, which undermines optimal decision-making in real-time operations. This point includes, although is not limited to, consistency of models used in different decision support tools along with its integration with plant information in real-time.

- Consider combinations of closed-loop and open-loop decision support tools to enhance decision-making processes in operations. In particular, how best leverage decision support tools at process plant and process network levels, as well as, offline tools e.g. offline simulation.

- Incorporate systematic accountability of uncertainties of multiple sources in the decision-making process in real-time. 


\subsubsection{INDUSTRIAL PLACEMENT}

Significant amounts of work were carried out to support this thesis, including an 18-months placement onsite working alongside Petronor staff at the refinery. This primarily, helped address an actual hydrogen network case study in its entirety. This preliminary work consisted of:

- Reviewing, updating and commissioning the open-loop RTO as it was a result of longterm collaboration between Petronor and University of Valladolid.

- Conducting periodical analysis and reports on RTO optimization policies of the hydrogen network against real operation figures.

- Performing test runs aimed at validation of equipment models and its impact on process unit operation.

The aforementioned tasks are underlying conditions that shaped the research and the resulting thesis in many ways, especially providing invaluable actual operational insights and off-the-book knowledge exchanged onsite with practitioners and operators. Please refer to Appendix D for a full report on the activities and outcomes of the industrial placement at Petronor. 


\subsection{AIM OF THE THESIS}

The main aim of this thesis is to study and develop a decision support tool for process network operators. For this purpose, I propose an integrated decision support framework for process network operators under uncertain conditions, which combines process information, model predictive control, optimization and simulation tools (see Fig.1.10). This decision support framework would sit above the basic control layer and exchange online process data through the historian database.

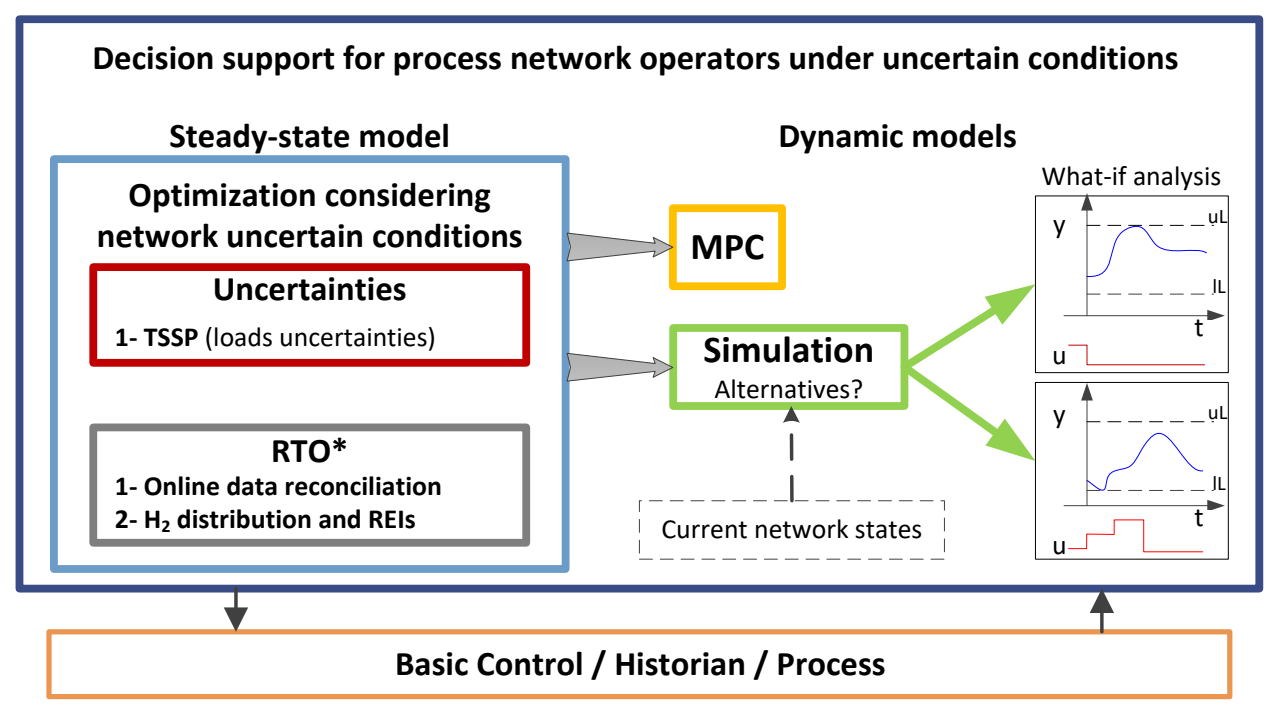

Fig. 1.10 - Information flow of the aimed decision support framework, comprising of: real-time optimization module (steady-state model) and real-time simulation module (dynamic model). RTO: real-time optimization. REI: resource efficiency indicator. MPC: model predictive control. * Existing deterministic formulation is used as starting point of this thesis.

Additionally, a refinery hydrogen process network case study is studied, considering process uncertainties and their impact on hydrogen network management, alongside pre-existing hydrogen management advanced process controller (i.e. DMC).

In particular, a modular decision support framework is proposed and developed as twofold comprising of:

- $\quad$ Real-time optimization module (steady-state model) 
- Real-time simulation module (dynamic models)

Real-time optimization module is based on deterministic and stochastic optimization methods (see Fig. 1.10, Steady-state model), which are focused on providing insights and clear information about optimal hydrogen network management for a given set of constraints. Real-time simulation is based on first-principle dynamic models (see Fig. 1.10 , dynamic models), which is capable of automatically collect plant data, estimate state evolution over time, and therefore forecast plant condition for a given set of control actions. These two modules are fully compatible with each other, as they are building blocks of a decision support framework to ensure proper data exchange between both modules. In Fig. 1.10 is a scheme showing the main components of the proposed decision support framework.

Simultaneously, another aim is to develop a dynamic simulation library, capable of successfully modelling hydrogen plants in the process network. This library is to be used as cornerstone of the real-time simulation module and fully compatible with real-time optimization module.

\subsubsection{SPECIFIC OBJECTIVES}

In line with the main aim, I have broken down this thesis into specific objectives for clarity. These are further reflected in the pieces of work presented throughout in the different chapters. The specific objectives are summarized in the following statements, and elaborated in the next sections accordingly:

- Design and develop a dynamic simulation library, based on first-principle models representative of phenomena taking place in crude oil refinery hydrogen networks and its uncertainties,

- Develop and analyze a real-time simulation application, which accounts for process change of condition in a real-time manner and enables assessment of multiple sets of expected conditions, 
- Develop and analyze a real-time optimization application, which accounts for hydrogen demand uncertainty across the process network in a risk-neutral and riskaverse manner,

- Propose a decision support framework set of design recommendations, analyzing its impact on efficient decision-making at different levels across business.

\subsubsection{DYNAMIC SIMULATION LIBRARY}

The objective is to design and develop a comprehensive first-principle process modelling library, accountable for the main process equipment, its dynamics and operations present in a crude oil refinery hydrogen network. The library should include components and operations which reflect plant behavior and topology realistically, such as stream splitters, mixers, valves and headers.

Another key function of the library is to efficiently exchange data of simulation execution files with a process plant historian, to effectively use online data.

Highlights of the dynamic simulation library are:

- Successfully modelling of hydrogen systems from process equipment up to process plants and network level,

- Ease of model modification and maintenance, due to models accounting for changes in the library in a straightforward manner,

- $\quad$ Stand-alone simulation execution, enabling user interface from Excel $^{\circledR}$ environment and other platforms,

- Made-for-purpose to underpin real-time simulation, especially when considering state estimation routines from online plant data, e.g. real-time reconciled simulation.

\subsubsection{REAL-TIME SIMULATION}

The objective is to develop a simulation featuring process forecasts in real-time, aimed at aiding operators in their decision-making process for future actions, especially helping them assess proposed actions from other decision support tools (e.g. RTO) or 
developing mitigation actions against unexpected changes at request. In order to achieve this, I propose an integrated state estimation and simulation application, accounting for past and current plant data and forecast future plant state for a given set of control actions. Additionally, this real-time simulation tool should feature results and model visualization in its user interface environment. This integrated application is named realtime reconciled simulation and the dynamic simulation library is its backbone, along with state estimation methods, reconciled parameters from the RTO and plant data from the historian database.

Highlights of real-time reconciled simulation are:

- Process analysis incorporating transition states, starting from current plant conditions,

- Assessment of multiple sets of control actions, considering uncertain values and explicit process economy,

- What-if analysis based on process data, either current or past,

- Ease of use at all process levels from equipment to process network,

- Combined with real-time optimization, enables validation of optimal solutions incorporating transitions onto the decision-making process.

\subsubsection{REAL-TIME OPTIMIZATION}

The objective around the real-time optimization module is to formulate and develop an application capable of resolving the hydrogen network management problem in real-time and on request considering stochastic uncertainties. This application should provide optimal hydrogen network management solutions for given sets of process constraints and hydrogen demand probability distribution function. Two approaches towards the decision-making process are presented and analyzed, risk-neutral and riskaverse formulation.

This module is underpinned by previous research work, which contributed with real-time data reconciliation network-wide and a base case for the deterministic optimization problem formulation (de Prada et al., 2017; Galan et al., 2018; Sarabia et al., 
2012). This is gratefully acknowledge, since it allowed further progress into stochastic programming for the crude oil refinery hydrogen network case study, and facilitated data collection via Excel ${ }^{\circledR}$.

Highlights of optimization under uncertainty are:

- Optimization considering hydrocarbon quality transition periods, which are subject to significant demand uncertainty,

- Works out multiple demand scenarios simultaneously, providing risk-neutral and riskaverse hydrogen network management advice,

- Combined with real-time reconciled simulation, enables fundamental analysis of main hydrogen management driving forces which can lead to improved management policies,

- Combined with deterministic formulation, supports decisions aimed at minimizing hydrogen demand uncertainty by quantification of so called value of stochastic solution (Birge and Louveaux, 2011), among other specific indices.

\subsubsection{DECISION SUPPORT FRAMEWORK}

The objective is to propose the architecture and functionality of a decision support framework in order to analyze and discuss its contributions towards better decision making, especially in control room contexts. For this purpose, a design criteria is developed and discussed based on the individual modules, previously presented. The focus is on the impact of multiple modules interconnected in the decision making process, especially when unexpected changes realize.

In addition, as a secondary objective, is the analysis of enterprise-wide decision support tools and how these relate with digital twins and fully informed decision-making processes.

Highlights of decision support framework analysis are: 
- Presents a general design criteria aimed at successfully developing decision support tools,

- Unleashes cost-effective use of data resources, focusing on hierarchically customized information to decision makers,

- Enables information cross checks of different modules for consistency in real-time or on demand, driving more solid decision making processes across the business,

\subsection{THESIS OUTLINE}

After the introduction (chapter one), the dynamic simulation library is described in chapter two, including a fundamental presentation of the first-principle equations incorporated in each piece of equipment modeled. In chapter three, the dynamic simulation library is applied alongside a state estimation method to develop real-time reconciled simulation, which is described. Moreover, two case studies are presented to demonstrate its usefulness in What-if analysis. Chapter four presents the module of optimization under uncertainty, this includes a full description of the stochastic formulation both risk-neutral and risk-averse, as well as most benchmarked stochastic optimization indices to compare deterministic formulation with the stochastic formulation proposed. Chapter five, deals with decision support framework concept and its impact on the decision making process. Furthermore, this chapter presents high level design criteria which are discussed focused on the role of a decision support framework, especially when subjected to change of conditions. In addition, some thoughts about decision support tools aimed at aiding enterprise-wide decision makers are discussed. Additionally, this chapter briefly presents reflections about the interactions of multiple decision support tools, potential synergies with the so called digital twin idea, towards a fully informed decision making. Chapter six, consists of a summary of the conclusions of the thesis, future challenges and open issues going forward are mentioned as well. This chapter finishes with a list of publications and contributions of this thesis. All references are listed in the last section. 
In addition, this thesis contains four appendixes, which bring complementary information and references on relevant sidetrack subjects. These are high level overviews provided for reference only, without distracting the reader from the main points. Appendixes are cited in the text where appropriate. 
2 DyNAMic Simulation LibRARY 



\section{Abstract}

This chapter describes and analyses a hydrogen network dynamic simulation library, which I developed during the course of my research project and is based on first principles occurring in typical process equipment within hydrogen networks of crude oil refineries. The library's main purpose is to account for the most representative dynamics observed in the processes involved, especially those dynamics with high impact at hydrogen network level. In this regard, the dynamics represented in the mathematical models are: hydrogen demands and light end generations, along with flows and hydrogen separation in separators. Another characteristic of the model is the use of two-component gas streams, one being hydrogen and the rest of species considered altogether as one pseudo-component called light ends. In addition, liquid hydrocarbon streams and mixed liquid-gas streams are defined.

The library consists of a set of components that model: process equipment (e.g. reactors, separators, pumps, etc.), process lines (e.g. stream mixers, stream splitters, etc.), hydrogen purification units and instrumentation (e.g. flowmeters, flow controllers, etc.). Library components have their respective first principle mathematical model, which help develop larger models using basic components as building blocks. Moreover, the dynamic simulation library enables developing tailored simulation models of hydrogen networks by interconnecting the appropriate components and setting up the parameters accordingly.

An additional library of actual process units and network headers is developed based on the main library. This library supports a network-wide model of Petronor's refinery hydrogen network.

In addition, it is discussed the utilization of the dynamic simulation library as the backbone of decision support tools with focus on the architecture. Furthermore, an example of architecture is proposed and analyzed towards the use of this library for developing enhanced simulation environments. The discussion ends with thoughts, based 
on an example, about leveraging dynamic simulation into real-time operation environments.

The chapter finishes with conclusions capturing the highlights of the dynamic simulation library and what it is useful for. 


\subsection{INTRODUCTION}

Process modeling and simulation of hydrogen networks requires the consideration of a broad range of operations in order to capture the whole set of units involved and their interconnections. Moreover, it is worth developing a library of hydrogen network objects rather than individual process units as closed objects in the modeling environment. The main advantage of the former being that, if the granularity of the library is appropriate, e.g. pieces of equipment or process sections, it enables modeling and simulation of multiplicity of plant and network configurations using the same library objects. This flexibility, along with its ease of use with respect to interconnecting objects are critical in further utilization of the library for modeling and simulation of real process plants.

Models based on first principle phenomena are of particular interest in building process libraries, since these rely on known relationships of variables given by general laws such as mass conservation, energy conservation, etc. Therefore, libraries based on first principle models can seamlessly be customized to build models of variable complexity using fundamental equations. Additionally, equation-based modeling programs are considered particularly efficient in multipurpose libraries due to their undefined resolution of the unknowns until a specific context is provided (model boundaries). See Appendix $A$ for a brief introduction on simulation fundamentals addressing different modeling approaches. A thorough survey on the upsides and downsides of equation-based (a.k.a. acasual) and casual modeling is provided by Schweiger et al. (2020). I would suggest Fritzson (2009, chap. 1) for a good introduction into acasual modeling and simulation.

In this section a detailed description of the components and architecture of a dynamic hydrogen network library is provided. The first principle equations are disclosed for the main components programmed as well as its corresponding codes. The library is programmed on EcosimPro ${ }^{\circledR} /$ PROOSIS $^{\circledR}$ (EA Internacional, 2019a), which is an object 
oriented equation-oriented modeling and simulation software. For a comprehensive introduction on EcosimPro ${ }^{\circledR} /$ PROOSIS $^{\circledR}$ refer to Vázquez et al. (2010).

The remaining of the chapter is broken down in the following sections. After the introduction in section 2.1, the objective of the dynamic simulation library is stated and formalized in sections 2.2. In section 2.3 the methodology is described, disclosing the first principle equations considered in the main components of the hydrogen network. Then the library itself is described in section 2.4, disclosing the actual code behind elements such as ports, components and process units. The next section (2.5) discusses the utilization and usability of the library as is built. The chapter ends with conclusions.

\subsection{OBJECTIVE}

The aim is to develop an efficient dynamic simulation library able to support dynamic models of refinery hydrogen process networks.

The library should account for the most remarkable process dynamics, such as hydrogen demand and light ends production changes, as well as separation phenomena downstream reactors. Therefore, process equipment and other components of the library would serve as building blocks of more complex models from individual process plants up to network level.

Additionally, the models should be useful in decision-making processes either in a stand-alone fashion or integrated within external environments, such as decision support frameworks. Ultimately, these would support decision makers by means of dynamic simulation, especially under changing process conditions.

\subsection{Modeling}

Any process system subject to mathematical modeling has to be adequately translated into equations, to successfully simulate actual phenomena to a satisfactory 
degree of accuracy. Moreover, decisions are made on how deep these phenomena representation should go and which hypothesis should hold for the model to be sufficiently accurate, ultimately consolidating the scope of the model.

In this case, liquid hydrocarbon streams are characterized by bulk properties (density, molecular weight and flowrate), and hydrogen should be distinguished from the rest of chemical species present for gas streams (2.1-2.4). Furthermore, mixed gas and liquid streams are represented simply by combining both gas and liquid properties. Interactions between gas and liquids, other than solubility, are neglected in the model for the sake of simplicity.

In addition, operations that directly affect hydrogen balance are considered separately. In fact, these operations can be summarized in the following processes: hydrogen demand of reactors (2.5-2.12), hydrogen solubility of separators (2.13-2.22) and gas purification of purification units (2.23-2.27). Other side processes such as light gases generation, total gas solubility in liquid hydrocarbons, molecular weight changes in purification units, are considered as well. Table 2.1 shows the nomenclature used in the equations set.

Table 2.1 - Nomenclature of variables and subscripts in set of equations 2.1 -2.27.

\begin{tabular}{|c|c|}
\hline$F$ & Gas flow, in $\mathrm{Nm}^{3} / \mathrm{h}$. \\
\hline G & Mixed gas and liquid stream flow, in $\mathrm{kmol} / \mathrm{h}$. \\
\hline$H C$ & Liquid hydrocarbon stream flow, in $\mathrm{m}^{3} / \mathrm{h}$. \\
\hline $\mathrm{ksol}_{\text {se, gasHC }}$ & Total gas solubility in hydrocarbons at separator se, in $\mathrm{Nm}^{3} / \mathrm{m}^{3} \mathrm{HC}$. \\
\hline $\mathrm{ksO}_{\mathrm{se}, \mathrm{H} 2 \mathrm{HC}}$ & $\mathrm{H}_{2}$ solubility in hydrocarbons at separator se, in $\mathrm{Nm}^{3} / \mathrm{m}^{3} \mathrm{HC}$. \\
\hline$k_{s s o l}$ se,MWLIG & $\mathrm{MW}_{\mathrm{LIG}}$ gas/liquid solubility coefficient, dimensionless. \\
\hline $\mathrm{ksOl}_{\mathrm{se}, \mathrm{H} 2 \mathrm{gas}}$ & $\mathrm{H}_{2}$ content in solubilized gas at separator se, in \%vol. \\
\hline$L M$ & Mol-Volume ratio for gases at $0 \circ \mathrm{C}$ and $1 \mathrm{~atm}, 22.414 \mathrm{Nm}^{3} / \mathrm{kmol}$. \\
\hline$M W$ & Molecular weight, in $\mathrm{kg} / \mathrm{kmol}$. \\
\hline$R D$ & Reactor demand $\left(R D_{H 2}\right)$ or generation $\left(R D_{L I G}\right)$, in $\mathrm{Nm}^{3} / \mathrm{h}$. \\
\hline
\end{tabular}




\begin{tabular}{|l|l|}
\hline$W$ & Mass flow, in $\mathrm{kg} / \mathrm{h}$. \\
\hline$X$ & H2 or LIG fraction in a gas stream, in \%vol. \\
\hline$\rho$ & Total molar fraction of a gas and liquid stream, in $\%$. \\
\hline$\tau$ & Density of a liquid stream, in $\mathrm{kg} / \mathrm{m}^{3}$. \\
\hline in & Hydraulic time constant in $\mathrm{h}$. \\
\hline $\mathrm{k}$ & The variable represents an inlet. \\
\hline$N$ & A node within the network. \\
\hline$o$ & Model node. \\
\hline out & The purified gas of a permeation membrane. \\
\hline$p g$ & The variable represents an outlet. \\
\hline$r$ & The gas purge of a permeation membrane. \\
\hline$r d$ & A reactor within the network. \\
\hline$s e$ & Specific demand ( $\mathrm{rd} \mathrm{H}_{2}$ ) or generation $(\mathrm{rd} \mathrm{LG})$, in $\mathrm{Nm}^{3} \mathrm{H}_{2} / \mathrm{m}^{3} \mathrm{HC}$. \\
\hline$z$ & A separator within the network. \\
\hline
\end{tabular}

\subsubsection{OVERALL MATERIAL BALANCES}

First and foremost, material balances of gas components and liquid streams are represented in process equipment and at stream nodes. Gas streams are modelled as bicomponent streams, containing molecular hydrogen $\left(\mathrm{H}_{2}\right)$ and a lump mixture of light hydrocarbons (LIG). The former is the main component and its molecular weight is a known constant value $\left(M W_{H 2}=2.01588 \mathrm{~kg} / \mathrm{kmol}\right)$. The latter, takes into account an aggregated share of methane, ethane, propane and any other gas components present in a gas stream. In sum, at each gas node the following first principles of mass conservation hold: total volumetric conservation (2.1), hydrogen mass balance (2.2), total mass balance 
by component $(2.3,2.4)$. Therefore, equations $2.1-2.4$ are held across all library components, and consequently by any model built using it. It should be noted that, all volumetric units of gases are at normal conditions i.e. $1 \mathrm{~atm}$ and $\mathrm{O}$ 드 (aka NPT), hence these are equivalent to gas moles balances. In Fig. 2.1 a generic node is represented with arrows indication flow directions (inlets and outlets) wrt node $N$.

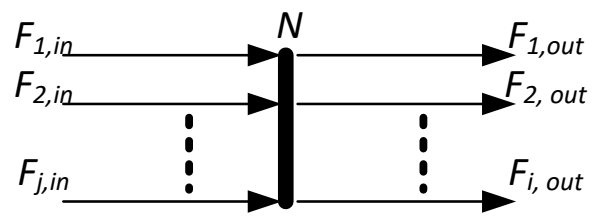

Inlet / Outlet stream properties:

$$
\mathrm{X}_{\mathrm{H} 2}, \mathrm{X}_{\mathrm{LIG}}, \mathrm{MW}, \mathrm{MW}_{\mathrm{LIG}}
$$

Fig. 2.1 - A generic node $N$, with arrows indicating flow directions, i.e. inlets (in) and outlets (out).

At all nodes $N$ :

$$
\begin{aligned}
& \sum_{i, \text { out }} F_{k, i}=\sum_{j, \text { in }} F_{k, j} \quad \forall k \in N \\
& X_{H 2, k} \cdot \sum_{i, \text { out }} F_{k, i}=\sum_{j, \text { in }} X_{H 2, k, j} \cdot F_{k, j} \quad \forall k \in N \\
& M W_{k} \cdot \sum_{i, \text { out }} F_{k, i}=\sum_{j, \text { in }} M W_{k, j} \cdot F_{k, j} \quad \forall k \in N \\
& 100 \cdot M W_{k}=M W_{H 2} \cdot X_{H 2, k}+\left(100-X_{H 2, k}\right) \cdot M W_{L I G, k} \quad \forall k \in N
\end{aligned}
$$

For the sake of simplicity equations of liquid streams are not presented, though these are shown in the code further in this chapter alongside a brief description. It should be noted that liquid stream nodes can be addressed as a particular case of gas streams, in the sense that liquid streams are mono-component instead of bi-component. 


\subsubsection{HYDROGEN DEMAND IN REACTORS}

Hydrogen is consumed in reactors as a consequence of hydrodesulfurization of hydrocarbons $(2.8,2.10)$, releasing light gases cracked from the feed as a side reaction $(2.9,2.11)$. Reactor inlet streams are mixed gas and liquid that suffer a chemical transformation, thus hydrogen is consumed and liquid hydrocarbons transferred to the gas phase $(2.7,2.13)$. First order hydraulic transition is considered as per 2.12. A generic reactor is featured in Fig. 2.2 for the sake of clarity of hydrogen demand equations (2.52.13).

Balances and phenomena represented by each equation in reactors $(r)$ is as follows:

- Overall moles balance of gases (2.5),

- Overall moles balance of hydrogen (2.6),

- Overall mass balance of gases (2.7),

- Chemical hydrogen demand (2.8),

- Chemical light ends generation (2.9),

- Rate of change of hydrogen demand per feed unit (2.10),

- Rate of change of light ends generation per feed unit (2.11),

- Liquid hydrocarbons balance (2.12),

- Overall mass balance of liquids (2.13).

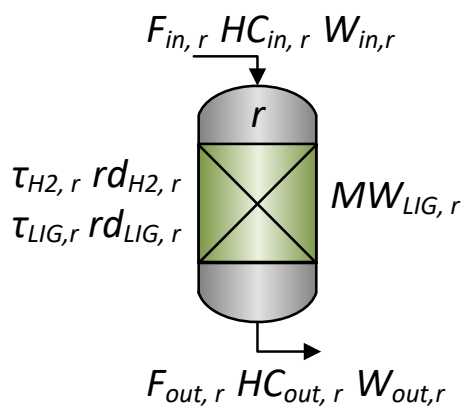

Inlet / Outlet stream properties:

$$
\mathrm{X}_{\mathrm{H} 2}, \mathrm{X}_{\mathrm{LG}}, \mathrm{MW}, \mathrm{MW}_{\mathrm{LIG}}
$$


Fig. 2.2 - Reactor schematic as support of hydrogen demand equations (2.5-2.13).

At all reactors $r$ :

$$
\begin{aligned}
& F_{\text {in }, r}=F_{\text {out }, r}+R D_{H 2, r}-R D_{\text {LIG }, r} \\
& F_{\text {in }, r} \frac{X_{\text {in }, r, H 2}}{100}=F_{\text {out }, r} \frac{X_{\text {out }, r, H 2}}{100}+R D_{H 2, r} \\
& F_{\text {in }, r} M W_{\text {in }, r}=F_{\text {out }, r} M W_{\text {out }, r}+R D_{H 2, r} M W_{H 2}-R D_{L I G, r} M W_{L I G, r} \\
& R D_{H 2, r}=H C_{i n, r} \cdot r d_{H 2, r} \\
& R D_{L I G, r}=H C_{i n, r} \cdot r d_{L I G, r} \\
& r d_{H 2, r}+\tau_{H 2, r} \cdot \frac{d\left(r d_{H 2, r}\right)}{d t}=r d_{H 2, r, s S} \\
& r d_{L I G, r}+\tau_{L I G, r} \cdot \frac{d\left(r d_{L I G, r}\right)}{d t}=r d_{L I G, r, s S} \\
& H C_{\text {in, } r}=\frac{d\left(H C_{\text {out }, r}\right)}{d t} \cdot \tau_{H C, r}+H C_{\text {out }, r} \\
& W_{H C, i n, r}=W_{H C, o u t, r}-R D_{L I G, r} \frac{M W_{L I G, r}}{L M}
\end{aligned}
$$

$\forall r \in$ Reactors

\subsubsection{SOLUBILITY PHENOMENA}

Mixed gas and liquid streams feed separators, where gas and liquids split into gas and mixed gas and liquid outlets (high or medium pressure separators) or gas and liquid outlets (low pressure separators). This separation in actual plants implies more than one piece of equipment, including knock out drums, strippers and condensers. In spite of that, 
pressure change is the main driver of the separation in all cases, which results in a gas stream outlet (high pressure side) and mixed gas and liquid stream outlet (medium or low pressure side). High or medium pressure separators are intermediate process equipment with a mixed gas and liquid outlet, while low pressure separators produce a final liquid product (without dissolved gases) typically pumped to storage outside plant battery limits.

Balances and phenomena represented by each equation in separators (se) is as follows:

- $k_{s o l} l_{s e, g a s H C}$ is defined as the solubility constant of gases in liquid hydrocarbons (2.14),

- $\mathrm{ksol}_{\mathrm{se}, \mathrm{H} 2 \mathrm{HC}}$ is defined as the solubility constant of hydrogen in liquid hydrocarbons (2.15),

- $\mathrm{ksol}_{\text {se,MWLIG }}$ is defined as the ratio of $M W_{L I G}$ of the gas outlet over $M W_{L I G}$ of the other outlet (2.16),

- $\mathrm{ksol}_{s e, \mathrm{H} 2 \mathrm{gas}}$ is defined as the ratio of $\mathrm{ksol}_{\mathrm{se}, \mathrm{H} 2 \mathrm{HC}}$ over $\mathrm{ksol}_{s e, \text { gasHC }}$ (2.17),

- Overall moles balance of gas (2.18),

- Overall moles balance of hydrogen (2.19),

- Overall mass balance of gases (2.20),

- Liquid hydrocarbons balance (2.21),

- Rate of change of $k s o l_{\text {se,gasHC }}$ (2.22),

- Rate of change of $k \mathrm{sol}_{\mathrm{se}, \mathrm{H} 2 \mathrm{HC}}$ (2.23).

A generic high pressures separator (se) is featured in Fig. 2.3 for the sake of clarity of equations 2.14 to 2.23 . 


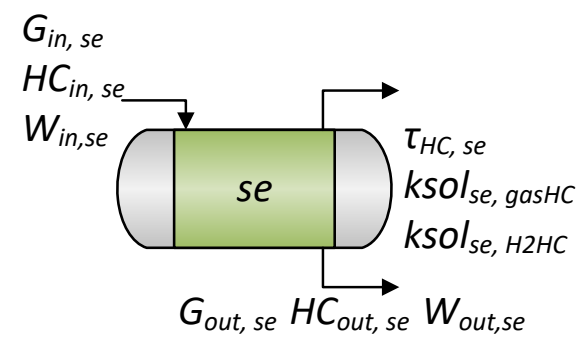

Inlet / Outlet stream properties:

$\mathrm{X}_{\mathrm{H} 2}, \mathrm{X}_{\mathrm{LIG}}, \mathrm{MW}, \mathrm{MW}_{\mathrm{LIG}}$

Fig. 2.3 - High pressure separator schematic as per equations 2.14 to 2.23 .

At all separators se, (2.21-23) do not apply to low pressure separators:

$$
\begin{aligned}
& k \text { sol }_{\text {se,gasHC }}=\frac{L M \cdot G_{\text {out }, \text { se }}\left(Y_{\text {out }, \text { se }, H 2}+Y_{\text {out }, \text { se }, L I G}\right)}{G_{\text {out }, \text { se }} \cdot Y_{\text {out }, \text { se }, H C}\left(\frac{M W_{H C}}{\rho_{H C}}\right)} \\
& k s s o l_{s e, H 2 H C}=\frac{L M \cdot G_{\text {out }, s e} \cdot Y_{\text {out }, \text { se }, H 2}}{G_{\text {out }, s e} \cdot Y_{\text {out }, s e, H C}\left(\frac{M W_{H C}}{\rho_{H C}}\right)}
\end{aligned}
$$

ksol $_{\text {se,MWLIG }}=\frac{M W_{\text {Fout }, \text { se }, \text { LIG }}}{M W_{\text {Gout }, \text { se }, \text { LIG }}}$

$$
k_{s o l}{ }_{s e, H 2 g a s}=\frac{k s o l_{s e, H 2 H C}}{k s o l_{s e, g a s H C}}
$$

$G_{\text {in }, s e}\left(100-Y_{\text {in }, H C}\right)=\frac{F_{\text {out }, \text { se }}}{L M}+G_{\text {out }, \text { se }}\left(100-Y_{\text {out }, s e, H C}\right)$

$$
G_{\text {in }, s e} Y_{\text {in, }, \mathrm{se}, \mathrm{H} 2}=\frac{F_{\text {out }, \text { se }}}{L M} X_{\text {out }, \mathrm{se}, \mathrm{H} 2}+G_{\text {out }, \mathrm{se}} Y_{\text {out }, \mathrm{se}, \mathrm{H} 2}
$$

$$
\begin{aligned}
& G_{\text {in }, s e}\left(100-Y_{\text {in }, H C}\right) M W_{\text {Gin }, s e} \\
& =\frac{F_{\text {out }, \text { se }} M W_{\text {out }, s e}}{L M}+G_{\text {out }, \text { se }}\left(100-Y_{\text {out }, \text { se }, H C}\right) M W_{\text {Gout }, \text { se }}
\end{aligned}
$$




$$
\begin{aligned}
& H C_{\text {in }, \text { se }}=\frac{d\left(H C_{\text {out }, ~ s e}\right)}{d t} \cdot \tau_{H C, \text { se }}+H C_{\text {out }, \text { se }}
\end{aligned}
$$

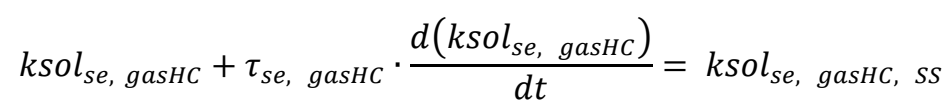

$$
\begin{aligned}
& k s o l_{s e, H 2 H C}+\tau_{s e, H 2 H C} \cdot \frac{d\left(k s o l_{s e, H 2 H C}\right)}{d t}=k s o l_{s e, H 2 H C, s S}
\end{aligned}
$$

$\forall$ se $\in$ Separators

\subsubsection{PURIFICATION PROCESSES}

Recycle gas streams contain higher impurities content than fresh make-up gas, and therefore purification units can be operated to purge impurities to fuel gas and increase hydrogen content of their permeate. Two types of purification units are considered, membranes and pressure swing adsorption (PSA). In particular, membranes are more complex for modelling purposes due to their variable purification performance (2.27).

Purification membrane units (see Fig. 2.4), operate under the principle of diffusion or permeation across a membrane. In general terms, a gas $\left(F_{i n, z}\right)$ fed into a purification membrane $(z)$ splits in two streams, permeate $\left(F_{o, z}\right)$ and purge $\left(F_{p g, z}\right)$. The permeate is concentrated in hydrogen due to a high affinity of this molecule across the membrane, mainly due to its small size compared with the rest of the species in the gas stream. The opposite holds for other species, which are concentrated in the purge. Membrane performance is essentially dependent on: feed and purge flow rates, hydrogen content in the feed, membrane material. Thus, for a given membrane (material unchanged), there are three main variables: $F_{i n, z}, F_{p g, z}$ and $X_{i n, H 2, z}$.

In overall, for membranes $(z)$ the following phenomena are considered for modeling:

- Overall moles balance of gases (2.24), 
- Overall moles balance of hydrogen (2.25),

- Overall mass balance of gases (2.26),

- Purification mass transfer (2.26),

- Impurities molecular weight conservation (2.28).

A schematic featuring the main variables and streams in a membrane unit is presented in Fig. 2.4 for the sake of clarity of equations 2.24 to 2.28.

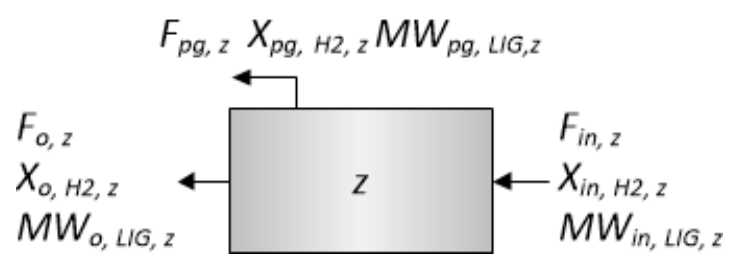

Fig. 2.4 - Schematic of membrane purification unit $z$ as per equations 2.24 to 2.28 .

At all permeation membranes $z$ :

$$
\begin{aligned}
& F_{i n, z}=F_{o, z}+F_{p g, z} \\
& F_{i n, z} \frac{X_{i n, z, H 2}}{100}=F_{o, z} \frac{X_{o, z, H 2}}{100}+F_{p g, z} \frac{X_{p g, z, H 2}}{100} \\
& F_{i n, z} M W_{i n, z}=F_{o, z} M W_{o, z}+F_{p g, z} M W_{p g, z} \\
& X_{o, z, H 2}=a \frac{F_{p g, z}}{F_{i n, z}}+b \cdot X_{i n, z, H 2}+c \\
& M W_{i n, z, L I G}=M W_{o, z, L I G}
\end{aligned}
$$

$\forall z \in$ Permeation membranes

where, $a, b$ and $c$ are membrane performance coefficients to match plant data to a particular membrane performance. These are addressed in section 2.4.4.3.1.1 Membrane purification units. 
Similarly, PSA units purify an inlet stream due to its adsorption affinity to an activated carbon bed. In particular, hydrogen flows through the bed of the PSA without being adsorbed, while the rest of the gas species are retained to some extent depending on their affinity to the bed pores. In addition, one PSA unit actually operates with several beds in a sequential fashion, coordinating feed intake and release (swing), even regeneration, in order for total flows to be accounted as continuous. As a result of this purified streams concentrations are very constant, regardless of other variables such as feed purity, feed or purge rate.

Pressure swing adsorption units hold the same overall balances as membranes $(2.24,2.25,2.26)$, while operating at fixed permeate purities and impurities molecular weight (2.27 and 2.28, do not apply). These units, in regard to mathematical modeling, can be considered as a particular case of membranes.

\subsection{LIBRARY DESCRIPTION}

The library under consideration comprises several levels of coding, which in the end generate a mathematical model of a process unit. A detailed description of all the components considered in the library is presented hereafter. The library is designed and developed on Ecosimpro ${ }^{\circledR} /$ PROOSIS $^{\circledR}$ (EA Internacional, 2019a), an object-oriented acasual simulation software, though once the model is compiled its use in other environments such as Excel is not only feasible but actually assessed. Moreover, the process equipment models generated where validated against plant data collected at Petronor refinery and its hydrogen network was used as case study across this thesis.

In addition, a high level description of the architecture and information flow of a stand-alone simulation application, called enhanced simulation framework, is provided. This includes a case study of real diesel hydrotreating process plant of Petronor refinery in Muskiz, Bilbao. 


\subsubsection{GENERALITIES}

The library is organized in components, which are objects containing a mathematical model. Different models connect to the outside environment and to each other through ports, which define connections of any model. Therefore, I used a combination of both to effectively develop a first-principles dynamic model library of refiner hydrogen networks. In addition, this library contains useful functions for programming simulations and optimization routines. These are particularly helpful for state and parameter estimation calculations, explained in detail in section 4.

The most general principles and variables are programmed at port level in the form of gas, liquid and mixed streams. Ports are used as well to define all the properties of each phase (gas and liquid), such as density, flowrate, molecular weight, and so on.

In gas streams, mole and mass relation of $\mathrm{H}_{2}$ and LIG is defined at port level, since is a principle that holds across components for any given model build with this library. A detailed description of each port is presented further in the text.

Liquid streams consider a single phase of hydrocarbons (HC) with its essential properties and how they relate to one another. Mixed streams account gas and liquid properties separately and altogether where that is meaningful, for example, total mass flow.

Apart from the aforementioned, it is worth noticing that some global constants are included in the code as known data for ease of programming. These are listed in Fig 2.1 and comprise ideal gas constant $(R), \mathrm{H}_{2}$ molecular weight (mwh2), liter-mole ratio at normal pressure and temperature $(L M)$. Table 2.2 presents the nomenclature used in the code, it has to be noticed that it has differences with the nomenclature of Table 2.1 due to typing restrictions of coding.

Detailed equations, along with the code of the main library features, are described in the following sections. 


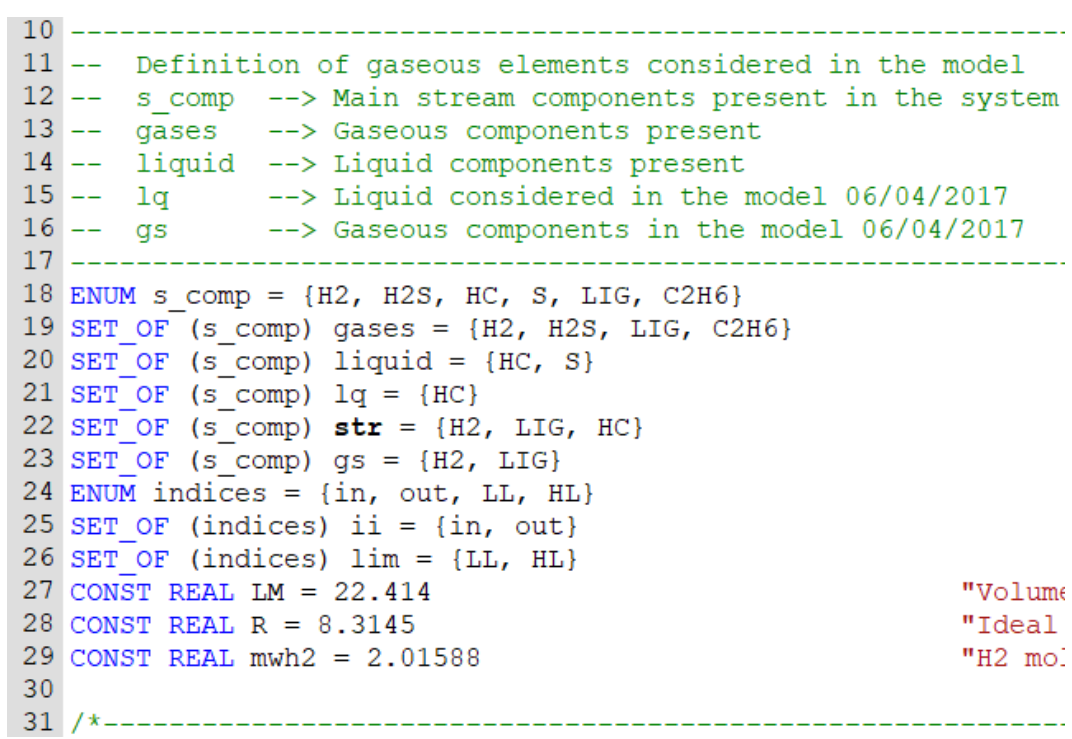

Fig. 2.1 - General constants and indices used across the library.

Table 2.2 - Dynamic simulation library's nomenclature. Symbols and descriptions used in the code presented.

\begin{tabular}{|l|l|}
\hline Variable & Description \\
\hline$W$ & Gas mass flow in $\mathrm{kg} / \mathrm{h}$. \\
\hline$F$ & Gas volumetric flow in $\mathrm{Nm}^{3} / \mathrm{h}$. \\
\hline$F_{-} g s[]$ & Gas component volumetric flow vector in $\mathrm{Nm}^{3} / \mathrm{h}[\mathrm{H} 2, \mathrm{LIG}]$. \\
\hline$y[]$ & Component molar in $\%[\mathrm{H} 2, \mathrm{LIG}]$. \\
\hline$r h o$ & Gas density in $\mathrm{kg} / \mathrm{Nm}^{3}$ \\
\hline$w$ & Gas molecular weight \\
\hline$w i$ & Light gases molecular \\
\hline$M h c$ & Molar flowrate of liquid in $\mathrm{kmol} / \mathrm{h}$. \\
\hline$w h c$ & Molecular weight in $\mathrm{kg} / \mathrm{kmol}$. \\
\hline$W h c$ & Mass flow of liquid in $\mathrm{kg} / \mathrm{h}$. \\
\hline Fhc & Volumetric flow of liquid in $\mathrm{Nm} / \mathrm{h}$. \\
\hline$r h o h c$ & Density of liquid in $\mathrm{kg} / \mathrm{m}^{3}$. \\
\hline$r_{-} F F h c$ & Gas-liquid ratio. \\
\hline Mtot & Total molar flow in $\mathrm{kmol} / \mathrm{h}$. \\
\hline$M x[]$ & Component molar flow in $\mathrm{kmol} / \mathrm{h}$. \\
\hline$x[]$ & Total molar fraction in $\%$. \\
\hline
\end{tabular}




\begin{tabular}{|c|c|}
\hline dir & Allowed gas flow direction variable, values 1 or 2 . \\
\hline$d r w i$ & Generated LIG molecular weight in kg/kmol. \\
\hline drrligh2 & LIG (generated) : $\mathrm{H}_{2}$ (consumed) ratio in $\mathrm{Nm}^{3} \mathrm{LIG} / \mathrm{Nm}^{3} \mathrm{H}_{2}$. \\
\hline tau_h2hc & Hydrogen demand first order time constant in $\mathrm{h}$. \\
\hline tau_lighc & LIG generation first order time constant in $\mathrm{h}$. \\
\hline$D R \_h 2$ & Reactor hydrogen demand in $\mathrm{Nm}^{3} / \mathrm{h}$. \\
\hline DR_lig & Reactor LIG generation in $\mathrm{Nm}^{3} / \mathrm{h}$. \\
\hline$D R \_w$ & LIG mass flow generation in $\mathrm{kg} / \mathrm{h}$. \\
\hline tau_hydr & Hydraulic time constant in $\mathrm{h}$. \\
\hline$D R \_w i$ & Molecular weight of LIG generated in kg/kmol. \\
\hline $\mathrm{rH} 2 \mathrm{HC}[]$ & Hydrogen : $\mathrm{HC}$ ration in $\mathrm{Nm}^{3} \mathrm{H} 2 / \mathrm{m}^{3} \mathrm{HC}$. \\
\hline drh2hc & Steady state hydrogen demand per volume of $\mathrm{HC}$ in $\mathrm{Nm}^{3} \mathrm{H}_{2} / \mathrm{m}^{3} \mathrm{HC}$. \\
\hline drlighc & Steady state LIG generation per volumen of $\mathrm{HC}$ in $\mathrm{Nm}^{3} \mathrm{H}_{2} / \mathrm{m}^{3} \mathrm{HC}$. \\
\hline dr_lighc & LIG generation per volumen of $\mathrm{HC}$ in $\mathrm{Nm}^{3} \mathrm{H}_{2} / \mathrm{m}^{3} \mathrm{HC}$. \\
\hline$d r \_h 2 h c$ & Hydrogen demand per volume of $\mathrm{HC}$ in $\mathrm{Nm}^{3} \mathrm{H}_{2} / \mathrm{m}^{3} \mathrm{HC}$. \\
\hline dr_wlighc & LIG generation per volumen of $\mathrm{HC}$ in $\mathrm{kg}$ LIG / m $\mathrm{m}^{3} \mathrm{HC}$. \\
\hline dr_ligh2 & LIG generation: $\mathrm{H}_{2}$ demand ratio in $\mathrm{Nm}^{3} \mathrm{LIG} / \mathrm{Nm}^{3} \mathrm{H}_{2}$. \\
\hline $\operatorname{gin}$ & Mixed gas and liquid stream inlet. \\
\hline gout & Mixed gas and liquid stream outlet. \\
\hline$f q$ & Quench stream. \\
\hline fout & Gas stream outlet. \\
\hline fin & Gas stream inlet. \\
\hline kswilig & LIG molecular weight gas-liquid split in $\mathrm{kg} \cdot \mathrm{kmol}^{-1} / \mathrm{kg} \cdot \mathrm{kmol}^{-1}$ \\
\hline tau_ks & Solubility coefficient first order time constant in $\mathrm{h}$. \\
\hline ks_h2gas & Ratio of Hydrogen-gas solubility in $\mathrm{HC}$ in \%1. \\
\hline ks_gasFhc & Gas solubility in $\mathrm{HC}$ in $\mathrm{Nm}^{3} / \mathrm{m}^{3} \mathrm{HC}$. \\
\hline ks_h2Fhc & Hydrogen solubility in $\mathrm{HC}$ in $\mathrm{Nm}^{3} \mathrm{H}_{2} / \mathrm{m}^{3} \mathrm{HC}$. \\
\hline ksgasFhc & Steady state gas solubility in $\mathrm{HC}$ in $\mathrm{Nm}^{3} / \mathrm{m}^{3} \mathrm{HC}$. \\
\hline ksh2Fhc & Steady state hydrogen solubility in $\mathrm{HC}$ in $\mathrm{Nm}^{3} \mathrm{H}_{2} / \mathrm{m}^{3} \mathrm{HC}$. \\
\hline tau_shp & High pressure separator hydraulic time constant in $\mathrm{h}$. \\
\hline fpurg & Purification unit purge stream. \\
\hline
\end{tabular}




\begin{tabular}{|l|l|}
\hline fperm & Purification unit purified stream. \\
\hline etha & Maximum operational purity in $\% \mathrm{H} 2$. \\
\hline perm_wi & Permeate impurities molecular weight in $\mathrm{kg} / \mathrm{kmol}$. \\
\hline d_wi & Impurities change coefficient in $\% 1$. \\
\hline __pgfin & Purification unit feed-purge ratio. \\
\hline del_y & Purification unit purity change in $\% \mathrm{H} 2$. \\
\hline
\end{tabular}

\subsubsection{PORTS}

Nomenclature of ports variables is presented in Table 2.1 alongside descriptions and their respective engineering units. These variables are used across the library in components' models at all levels, basically easing coding, programming and debugging processes.

\subsubsection{GAS STREAMS}

Gas streams (gas ports) have three degrees of freedom (DoF), which solve all their properties. These being: volumetric flowrate $(F)$, purity of one component $(y[])$, and molecular weight of impurities (wi).

The equations coded in gas stream ports are shown in Fig. 2.2, lines 21 to 37 . These reflect essential gas properties of bi-component streams (i.e. $H_{2}, L I G$ ).

This port and all components representing gas streams are in blue throughout the library, see Fig. 2.5. 


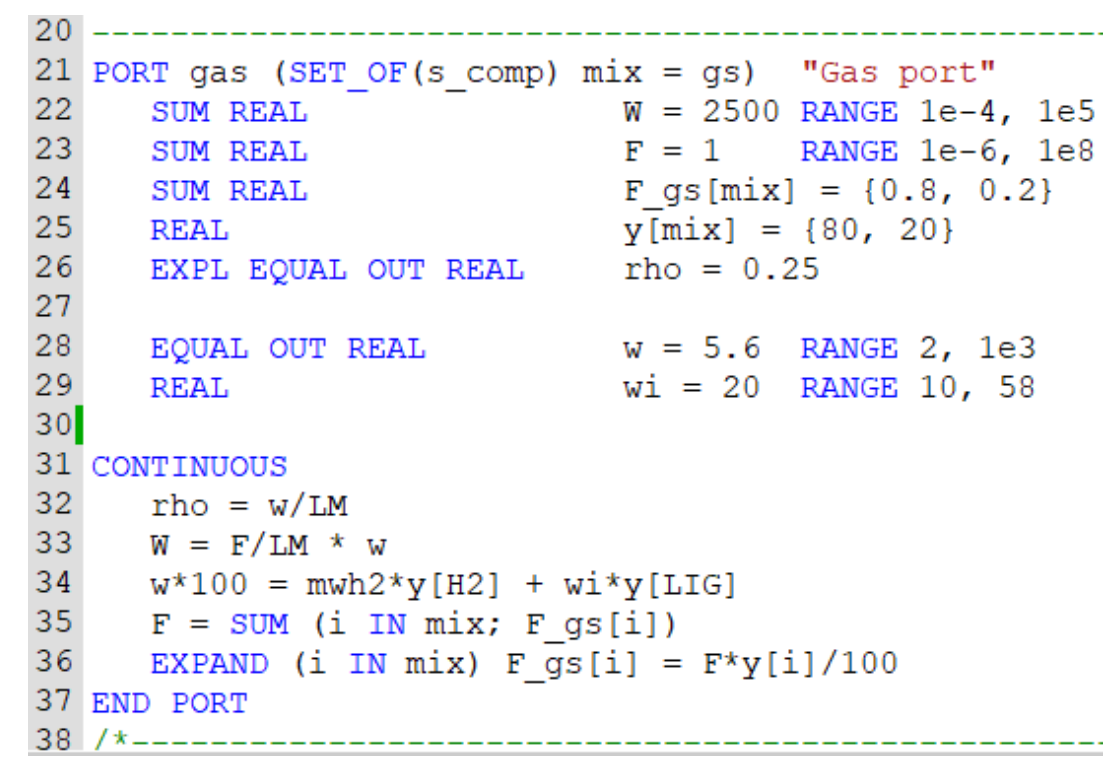

Fig. 2.2 - Equations coded in gas ports used in gaseous streams.

\subsubsection{LIQUID STREAMS}

Liquid streams (hc ports) have three degrees of freedom (DoF), which solve all their properties. These being: flowrate, density, and molecular weight.

The equations coded in liquid stream ports are shown in Fig. 2.3, lines 51 to 60. These reflect essential liquid properties for liquid hydrocarbon streams. Moreover, the link between generic gas variables is defined at this level in the continuous block (line 57). It is important to notice that flowrates (Whc, Fhc) are defined as SUM REAL (line 52), which automatically incorporates an addition of this variables when connected to another gas port. Similarly, intensive properties such as density (rhohc), molecular weight (whc) are conserved in outlet connections.

This port and all components representing liquid streams are in orange throughout the library, see Fig. 2.5. 


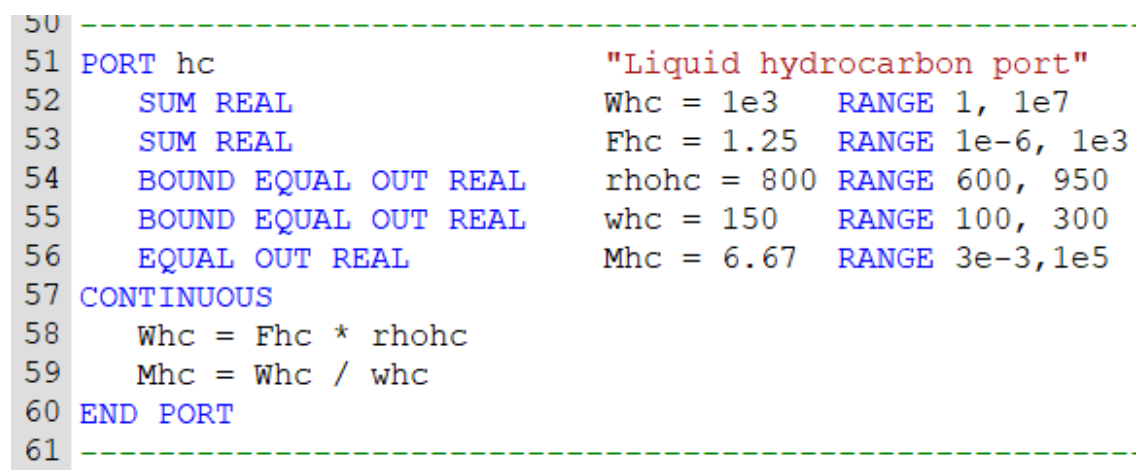

Fig. 2.3 - Equations coded in hc ports used in liquid hydrocarbon streams.

\subsubsection{MIXED GAS AND LIQUID STREAMS}

The library considers mixed gas and liquid streams as merged streams, essentially combining previously defined gas and liquid variables, see Fig. 2.4. Therefore, this streams have six DoF, three corresponding to gas and three to liquid. In addition, it accounts for overall composition, thus it comprises three components in total (i.e. $H C, H_{2}$, LIG). Overall variables are defined in Fig. 2.4 at lines 91 to 94, and linking equations coded in the continuous block in lines 107 to 111 . The complete code of this port, including its equations, is shown in Fig. 2.4.

This port and all components representing mixed gas and liquid streams are in green throughout the library symbols, see Fig. 2.5 . 


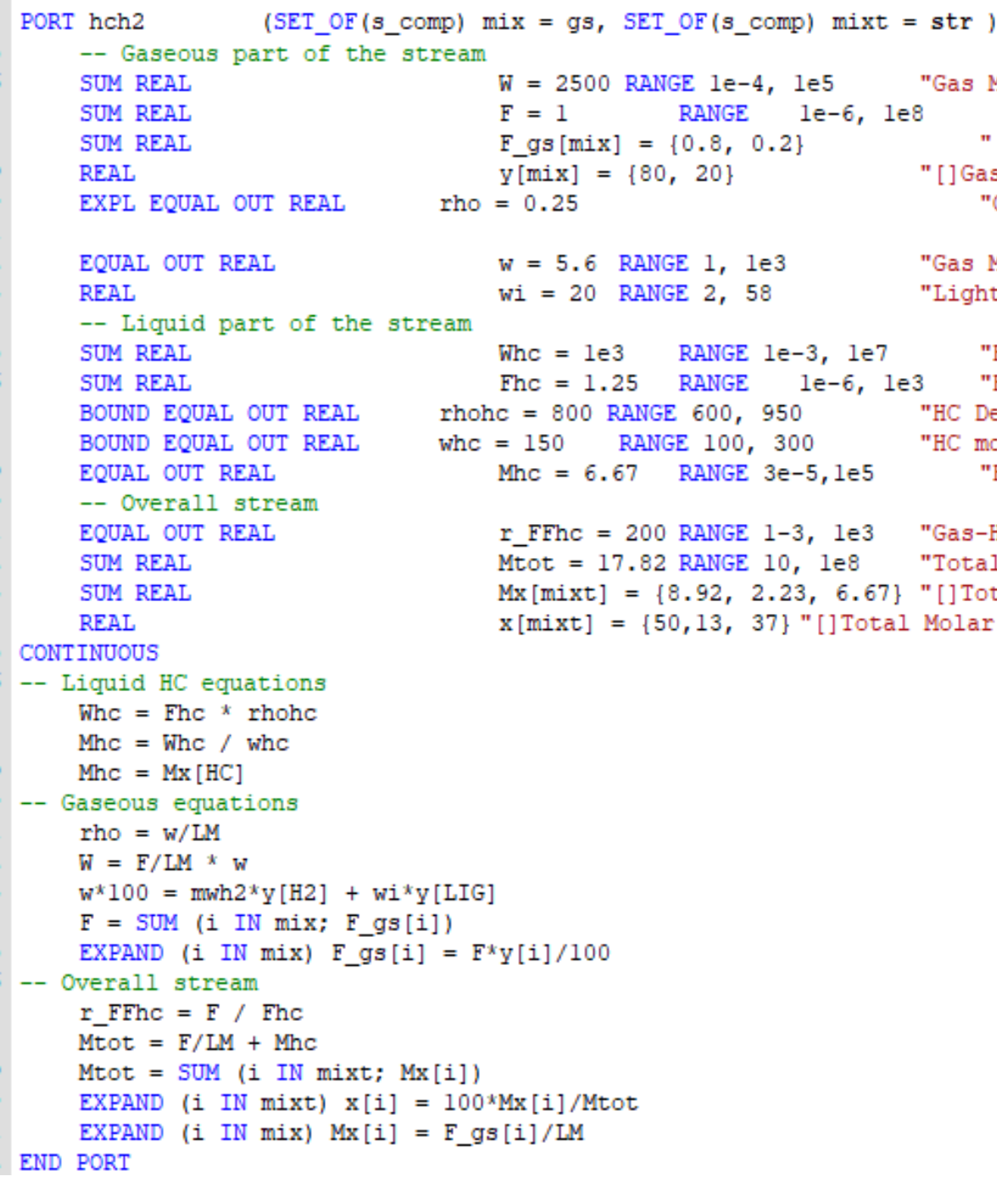

Fig. 2.4 - Equations coded in hch2 ports used in mixed liquid and gaseous streams.

\subsubsection{COMPONENTS}

Components are the second hierarchical level of building blocks of the dynamic library, right after the equations it selves. These are loosely defined as an enclosed mathematical model that can be solved for a given set of boundaries and initial conditions, including any algebraic initial values. Basic components represent well defined machinery or equipment, such as reactors, valves, columns, separators. Another family of components represents pipeline arrangements, such as stream mixers, stream splitters 
and headers. Ports are responsible for component-to-component connections, as well as, component-to-environment connections.

A graphic symbol is linked to each component, which follows the color code for ports as it is presented in Fig. 2.5. Equipment green-filled is distinguished from the rest since these incorporate dynamics in their model.

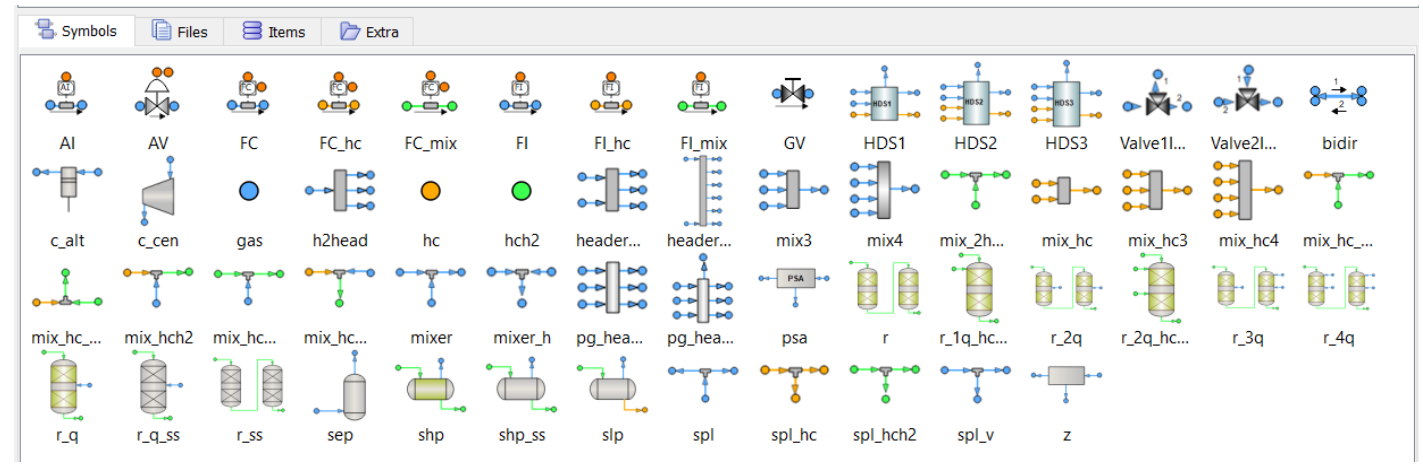

Fig. 2.5 - Dynamic simulation library symbols.

\subsubsection{MIXERS AND SPLITTERS}

Stream mixers and splitters are sorted by number of inlets and outlets, and type of fluid of each one (i.e. gas, liquid, mixed). As a general rule, mixers require full determination of all their streams but one, thereby DoF is as 2.29 to 2.31 , depending on the fluid under consideration. The same rationale applies to mixers with different stream types. Some examples of mixers are shown in Fig. 2.6 to Fig. 2.8.

$$
\begin{aligned}
& D o F_{\text {mixer,gas }}=3 \cdot(S-1) \\
& D o F_{\text {mixer }, h c}=3 \cdot(S-1) \\
& D o F_{\text {mixer }, \text { hch } 2}=6 \cdot(S-1)
\end{aligned}
$$

Where $S$ is the number of inlet streams of each fluid port type: gas, hc and hch2. 

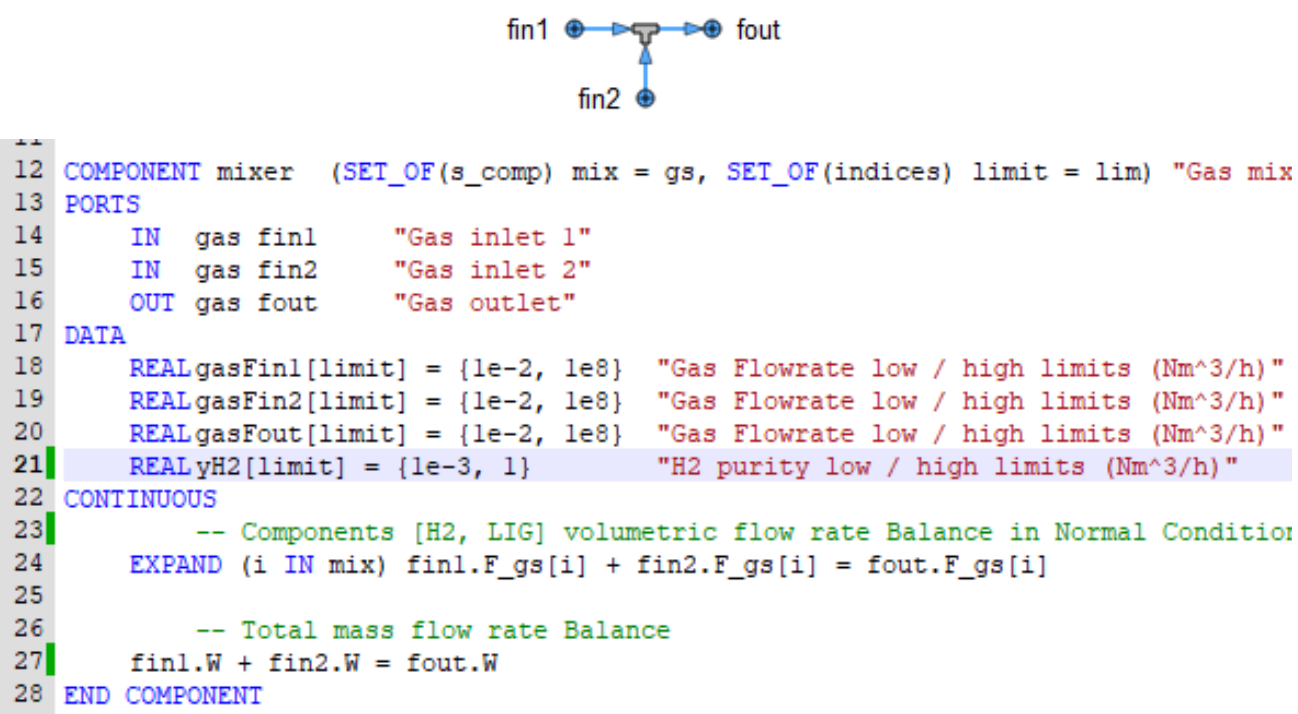

Fig. 2.6 - Two inlets, one outlet gas mixer.
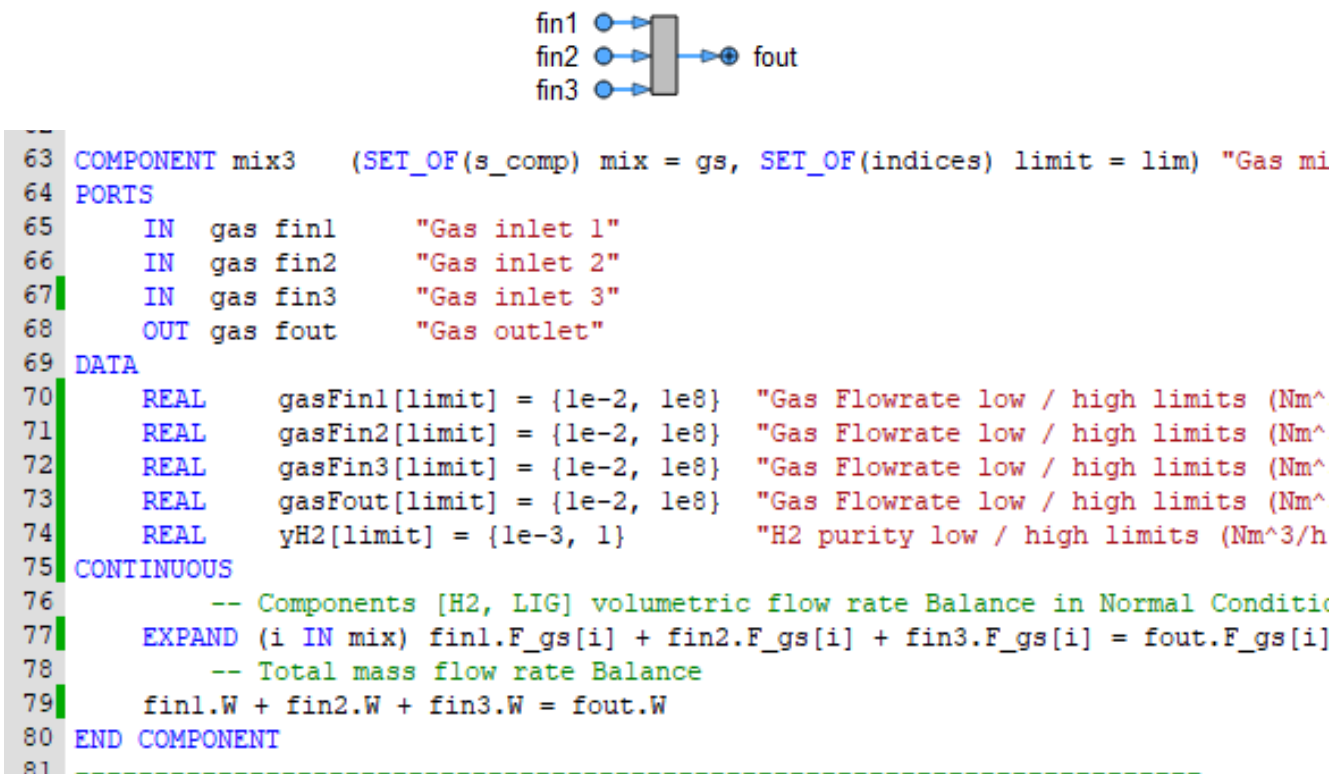

Fig. 2.7 - Three inlets, one outlet gas mixer. 


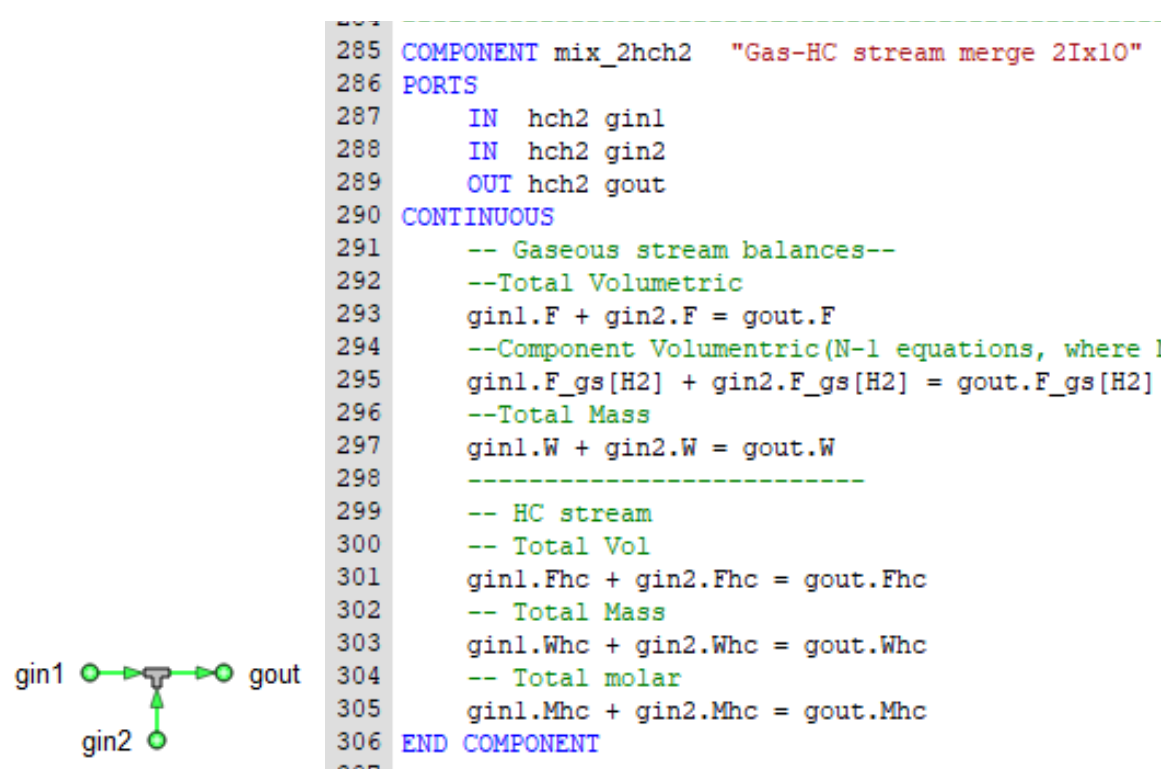

Fig. 2.8 - Two inlets, one outlet mixed gas and liquid mixer.

On the other hand, stream splitters require full information of the outlets flowrates except one and totally determined inlet stream. Therefore, their DoF is as in 2.32 to 2.34 , depending on the fluid under consideration.

$$
\begin{aligned}
& \text { DoF } F_{\text {splitter,gas }}=3-(S-1) \\
& D o F_{\text {splitter }, h c}=3-(S-1) \\
& D o F_{\text {splitter,hch } 2}=6-(S-1)
\end{aligned}
$$




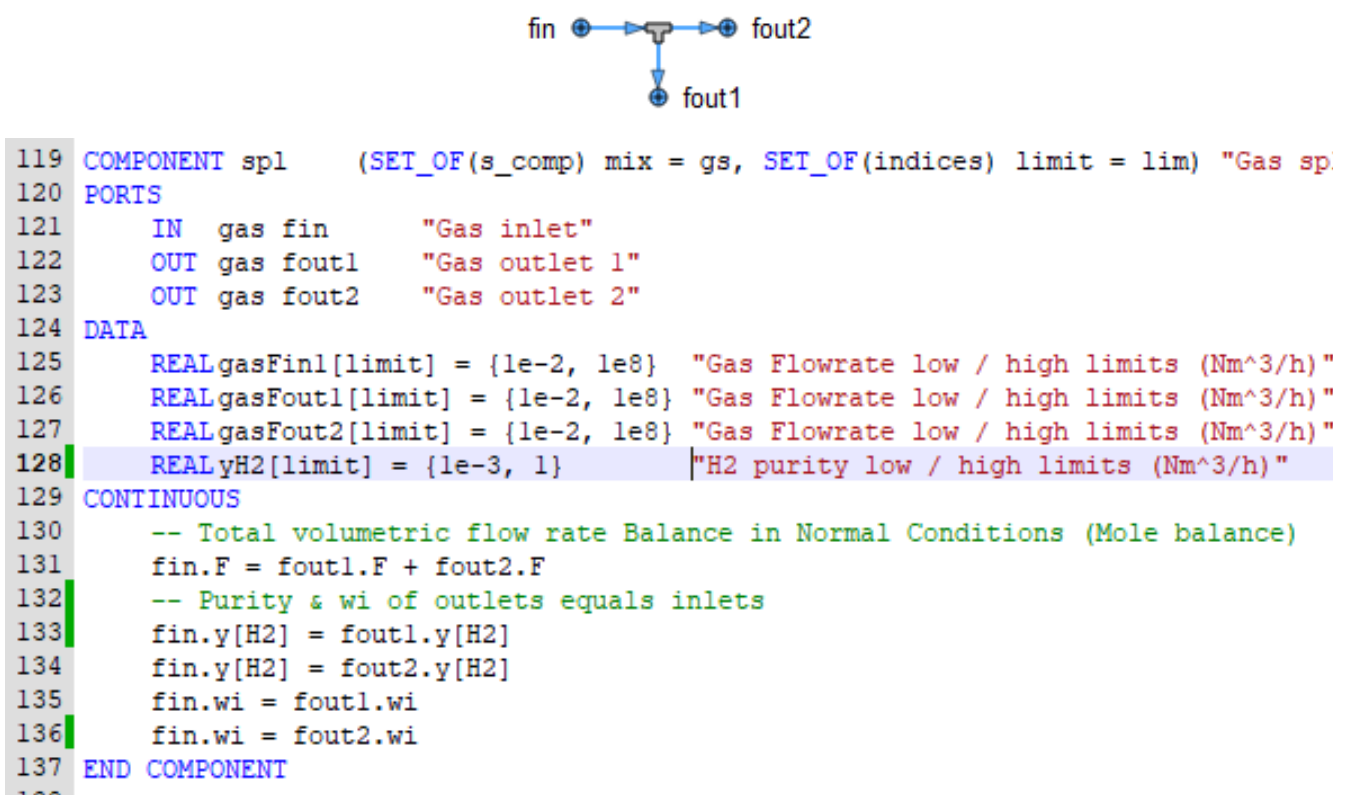

Fig. 2.9 - One inlet, two outlets gas splitter.

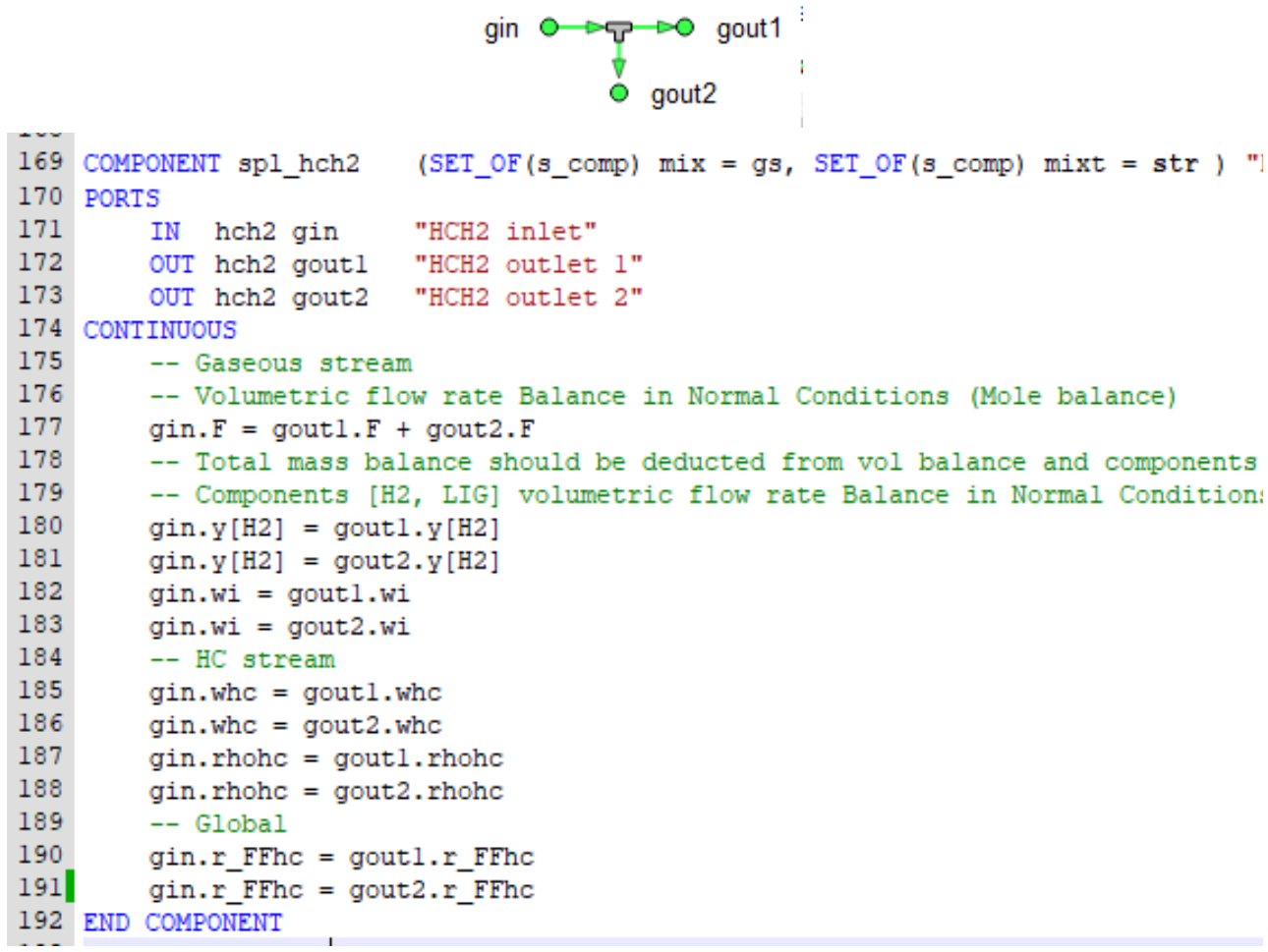

Fig. 2.10 - One inlet, two outlets mixed gas and liquid splitter. 


\subsubsection{BIDIRECTIONAL PIPELINE}

In order to represent pipelines that allow different flow directions a specific component is coded, which requires an additional integer variable to define flow direction (dir). In this form, an "if" clause works out the proper set of equations depending on the direction stated, the full code shown in Fig. 2.11. In addition, this component uses a minimum counter current flow as an auxiliary flow for computational coherence (see line 444 in Fig. 2.11), with negligible impact on results.

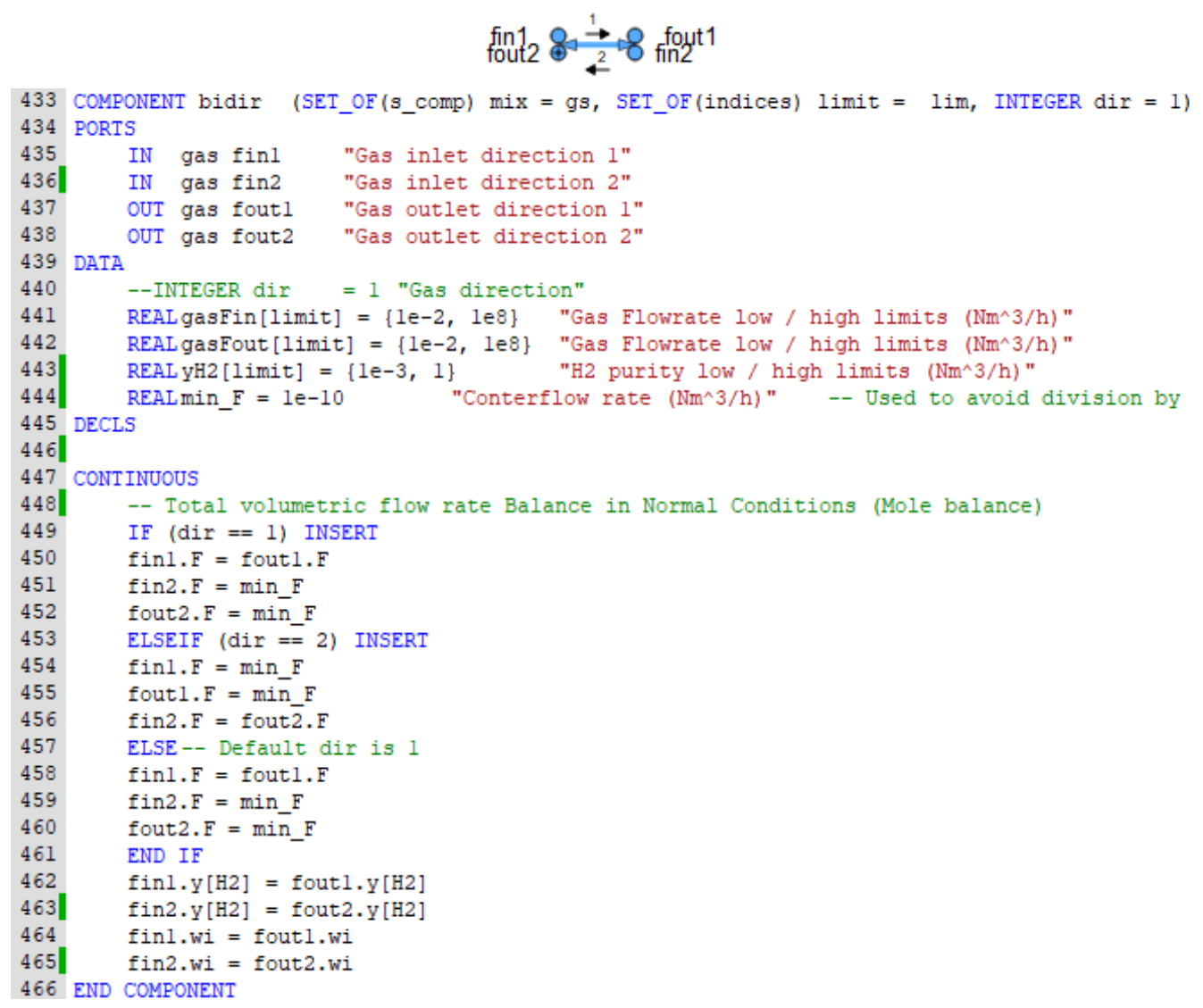

Fig. 2.11 - Bidirectional gas pipeline.

\subsubsection{PROCESS UNITS}

In this section the models of the following process units are described here-below, between brackets the component name in italics: 
- $\quad$ Single or multiple bed reactors without quench $(r)$,

- $\quad$ Single or multiple bed reactors with quench $(r)$

- Two-bed reactors with mixed gas and liquid quench (r_2q_hch2),

- High pressure separators (shp),

- Low pressure separators (s/p),

- Absorber columns (sep),

- Reciprocating (c_alt) and centrifugal (c_cen) compressors,

- Purification membrane (z),

- Pressure swing adsorption unit (psa),

- Flowmeter $(F I)$ and flow controller $(F C)$.

Although this is considered a sensible list of the most significant operation equipment currently present in the library, it is not exhaustive. In total, the library contains 54 operations modelled with symbols assigned (Fig. 2.5), which in turn provide enough flexibility to represent process units related in oil refinery hydrogen networks.

\subsubsection{REACTORS}

All reactors have inherited the code shown in Fig. 2.12, which sets fundamental balances applicable to all types of reactors in the library. In essence, reactors are responsible for the following phenomena, all line references refer to the code shown in Fig.2.12:

- first order dynamic in hydrogen demand where $d r_{-} h 2 h c$ is the state variable (line 59),

- first order dynamic in light ends generation where $d r$ lighc is the state variable (line 63),

- molecular weight change (line 65, 67),

- volumetric flow changes to cope with light ends generation (lines 73 to 75 ), therefore density change is assumed negligible (modo 1 ),

- density changes to cope with light ends generation (lines 76 to 78), therefore volumetric flow change is assumed negligible (modo 2, default), 
- first order dynamic in either volumetric flow where gout. Fhc is the state variable (line 80 , modo 2 ) or mass flow where gout. Whc is the state variable (line 74 , modo 1 )

- three time characteristic constants, tau_h2hc (hydrogen demand state, line 52), tau_lighc (light ends generation state, line 50) and tau_hydr (flowrate state, line 78 in modo 2 or line 74 in modo 1 )

Reactors have at least one mixed gas and liquid stream (gin.Fhc, DoF $=6$ ), three dynamic variables $\left(d r \_h 2 h c, d r \_l i g h c, g o u t . F h c\right)$, one outlet stream and may or may not have quenches of any stream type (gas quench is the most common). Therefore, the degrees of freedom of reactors without quench streams totals six. In the case of reactors with quenches, the degrees of freedom provided by the corresponding stream type should be added to the total DoF of the reactor.

The simplest reactor model is the single or multiple bed reactors without quench $(r)$ as shown in Fig. 2.13 (lines 85 to 95). This process unit has not quench stream, therefore accounts six DoF in total. Single bed reactors are common in simple hydrotreating process, or sometimes used as finishing reactors to remove impurities downstream a main reactor section.

Although the fundamental chemical and mass transfer phenomena are represented in all reactors, the library provides several options for better mimic plant processes. These other reactor options simply incorporate quench streams, which can be of any fluid type (i.e. gas, $h c$ or $h c h 2$ ) being gaseous the most widely used in hydrotreating processes. In Fig. 2.13 a reactor with one quench stream is presented $\left(r_{-} q\right.$, lines 99 to 112, DoF $\left.=9\right)$ and Fig. 2.14 shows the code of a two-bed reactor with mixed gas and liquid stream quenches $(\mathrm{DoF}=18)$. Both of them are very common and widely used in hydrotreating processes. 


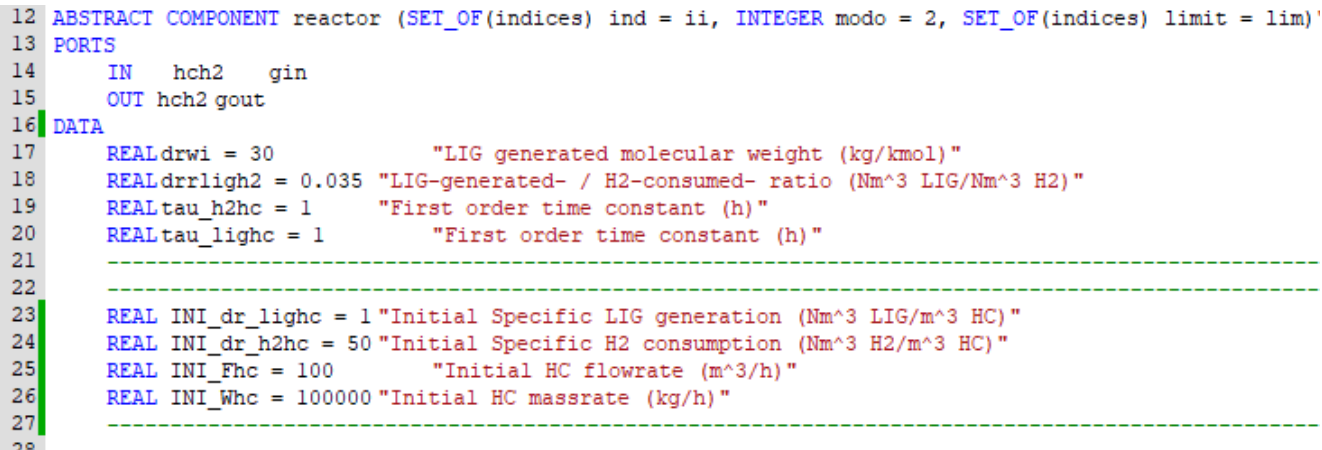

29 ----- Operational Limits -----------

30 REAL rh2hc IN [limit] $=\{100,600\}$ "Inlet Ratio H2/HC low / high limits (Nm^3 H2/m^3 HC)" DECLS

REAL rh2hc_OUT [limit] $=\{100,600\}$ "Outlet Ratio H2/HC low / high limits (Nm^3 H2/m^3 HC) REAL yh2 $[1 \overline{i m i t}]=\{1,100\} \quad$ H2 content low / high limits $\left(\frac{\%}{\%}\right)$ "

EXPL REAL DR_h2 = 5000 "H2 consumption in reactor $\left(\mathrm{Nm}^{\wedge} 3 / \mathrm{h}\right)$ "

EXPL REAL DR_lig $=1000$ "LIG vol flow generation in reactor $\left(\mathrm{Nm}^{\wedge} 3 / \mathrm{h}\right)$ "

EXPL REAL DR $w=1340$ "LIG mass flow generation in reactor $(\mathrm{kg} / \mathrm{h})$ "

BOUND REAL tau_hydr $=0.8$ "Hydraulic time constant $(\mathrm{h})$ "

REAL DR wi $=3 \overline{0} \quad$ "LIG generated molecular weight $(\mathrm{kg} / \mathrm{kmol})$ "

REAL $\mathrm{rH} 2 \mathrm{HC}\left[\right.$ ind] $=500$ "Ratio $\mathrm{H} 2 / \mathrm{HC}$ in operation $\left(\mathrm{Nm} \wedge 3 \mathrm{H} 2 / \mathrm{m}^{\wedge} 3 \mathrm{HC}\right)$ "

BOUND REAL drh2hc $=50$ "Specific H2 consumption ( $\mathrm{Nm}^{\wedge} 3 \mathrm{H} 2 / \mathrm{m}^{\wedge} 3 \mathrm{HC}$ ) "

BOUND REALdrlighc $=1$ "Specific LIG generation ( $\left.\mathrm{Nm}^{\wedge} 3 \mathrm{LIG} / \mathrm{m}^{\wedge} 3 \mathrm{HC}\right)$ "

REAL dr_h2hc $=50$ "Specific $\mathrm{H} 2$ consumption ( $\left.\mathrm{Nm}^{\wedge} 3 \mathrm{H} 2 / \mathrm{m}^{\wedge} 3 \mathrm{HC}\right)$ "

REAL dr_lighc $=10$ "Specific LIG generation in vol $\left(\mathrm{Nm}^{\wedge} 3 \mathrm{LIG} / \mathrm{m}^{\wedge} 3 \mathrm{HC}\right)$ "

REAL dr wlighc $=15$ "Specific LIG generation in mass $\left(\mathrm{kg} \operatorname{LIG} / \mathrm{m}^{\wedge} 3 \mathrm{HC}\right)$ "

REAL dr_ligh2 = 10 "LIG-generated- / H2-consumed- ratio ( $\left.\mathrm{Nm}^{\wedge} 3 \mathrm{LIG} / \mathrm{Nm}^{\wedge} 3 \mathrm{H} 2\right)$ "

CONTINUOUS

DR_wi $=$ drwi

dr_ligh2 = DYN H2NETWORK. divx (dr lighc, dr h2hc, 0.01)

dr_lighc + tau_lighc * dr_lighc' ${ }^{-}=$drlighc

-- First order dynamic

$\mathrm{dr} h 2 \mathrm{hc}+\operatorname{tau} \mathrm{h} 2 \mathrm{hc} * \mathrm{dr} \mathrm{h} 2 \mathrm{hc} \mathrm{c}^{\prime}=\mathrm{drh} 2 \mathrm{hc}$

-- $\mathrm{H} 2 \mathrm{HC}$ ratio definition -- ind $=i$ for inlet and ind $=0$ for outlet

gin. $\mathrm{F}$ gs $[\mathrm{H} 2]=\mathrm{rH} 2 \mathrm{HC}[$ in $] *$ gin. Fhc

gout. $\bar{F}$ _gs $[\mathrm{H} 2]=\mathrm{rH} 2 \mathrm{HC}[$ out $]{ }^{*}$ gout. Fhc

-- H2 consumption

DR_h2 $=$ dr_h2hc* gout. Fhe

-- LIG generation

-- In Volume

DR lig $=$ dr_lighc $*$ gout. Fhc

-- Mass generation ratio

dr_wlighc $=$ dr_lighc * DR_wi/LM

-- In mass

DR_w $=$ dr_wlighc * gout. Fhc

$/^{*}--$ Assumptions for the volume and mass conservation in the reactor are different for each

--- modo $=1,2$

-- modo = 1 assumes rhohc change is neglegible in the reactor, though Fhc changes to reflect mas!

-- modo $=2$ assumes Fhc change is neglegible in the reactor, though rhohc changes to reflect ma IF (modo $==1$ ) INSERT

gin. Whe $=$ (tau hydr * gout. Whe' + gout. Whc $)+$ DR w

gin. rhohc $=$ gout .rhohc

ELSEIF (modo $==2$ ) INSERT

gin. Whe $=$ gout. Whe + DR_W

gin. Fhe $=$ tau_hydr ${ }^{\prime}$ gout. Fhc' + gout. Fhc

END IF

gout.Mx $[\mathrm{HC}]=$ gin.Mx $[\mathrm{HC}]-\mathrm{DR}$ lig/LM // Considers molec weight change of HC stream

1 END COMPONENT

Fig. 2.12 - Abstract reactor code, basic equation and variables support across all reactors in the dynamic library. 


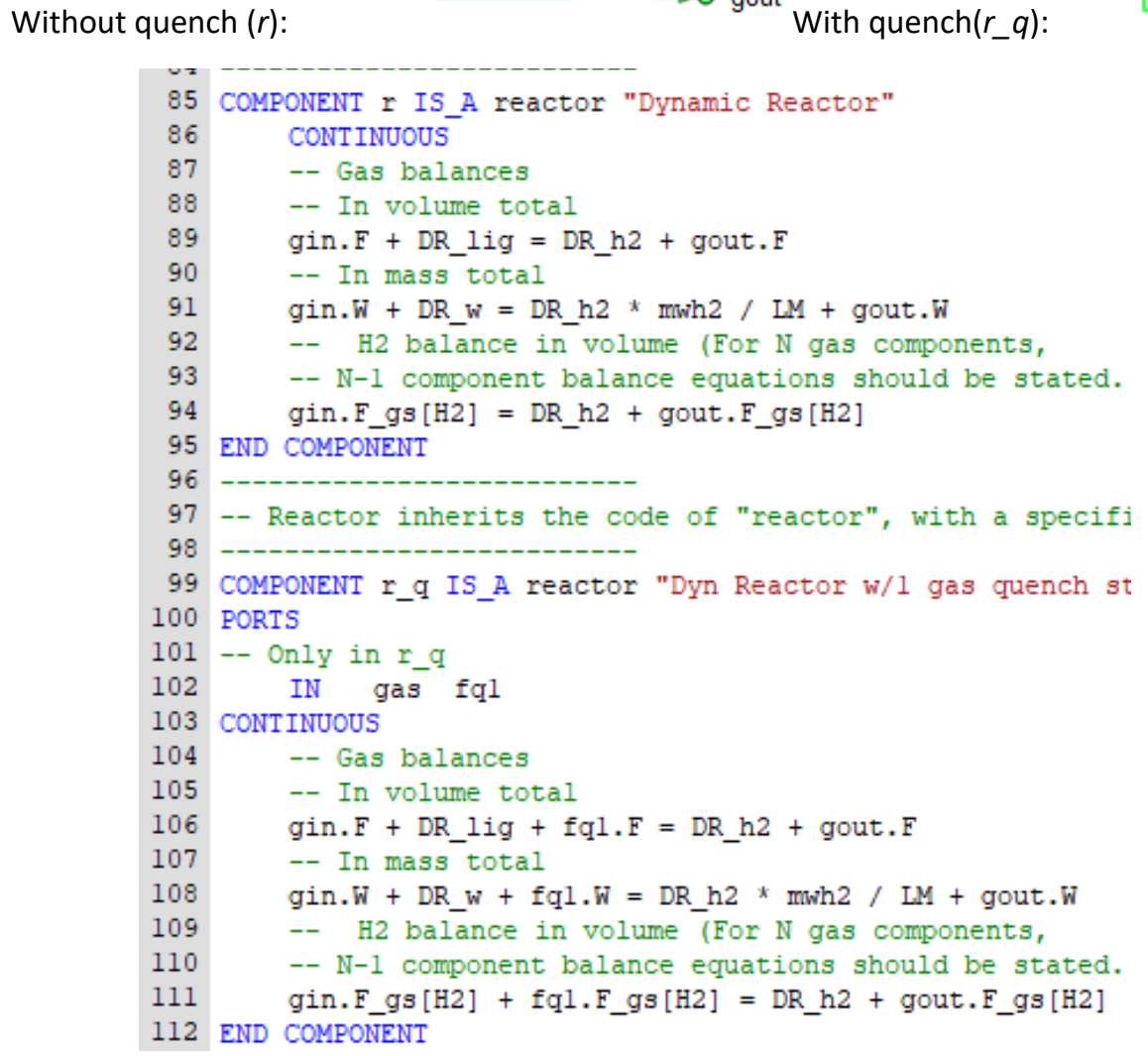

Fig. 2.13 - Single bed reactor component $(r$, line 85 , DoF $=6)$, followed by a gas quenched reactor $\left(r \_q\right.$, line 99, DoF = 9). 


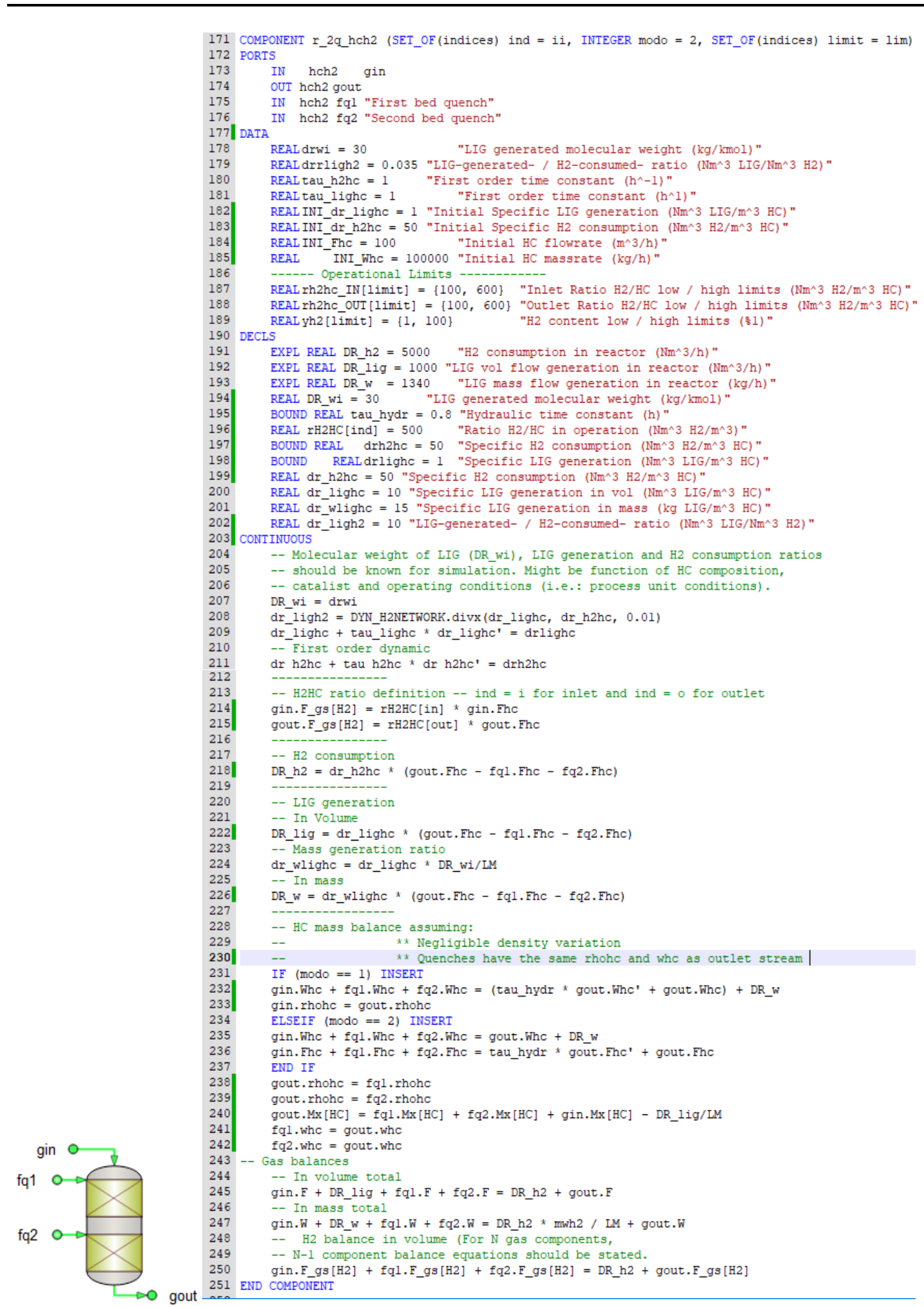

Fig. 2.14 - Mixed gas and liquid quenched reactor unit (DoF = 18). 


\subsubsection{SEPARATORS}

High pressure (HPr) and low pressure (LPr) separation units are modelled, aimed at removing undissolved gas from a mixed gas and liquid stream. High pressure separators are typically the first equipment downstream the reactor, therefore subject to reactor section pressure loop. Since pressure is controlled and specified by the process and catalyst technology, for the purpose of the simulation is assumed fixed. Therefore, separators fundamental phenomena are represented by equations shown in Fig. 2.15 from line 411 to line 425 (shp, HPr) and in Fig. 2.16 (left) from line 496 to line 502 (s/p, $\mathrm{LPr}$ ). In the case of HPr, more complex phenomena are described since the mixed liquid and gas stream retains an amount of hydrogen and light ends proportional to the solubility of those gases in the liquid hydrocarbons. In the end, the following behaviors are considered, all line references refer to the code shown in Fig.2.15:

- first order dynamic in liquid HC where gout.Fhc is the state variable (line 411),

- first order dynamic in gas solubility in liquid HC where ks_gasFhc is the state variable (line 419),

- first order dynamic in hydrogen solubility in liquid HC where $k s_{-} h 2 F h c$ is the state variable (line 420),

- impurities molecular weight distribution coefficient, kswilig, over gas and liquid phases (line 425).

Given the aforementioned system of equations for separators, these components have six degrees of freedom (DoF $=6)$.

It should be noticed that LPr separators behave as a particular case of HPr, since the former are modelled as steady state pieces of equipment. Additionally, negligible solubility of hydrogen in liquid HC is considered at LPr conditions, something that has been confirmed by experimental data. 

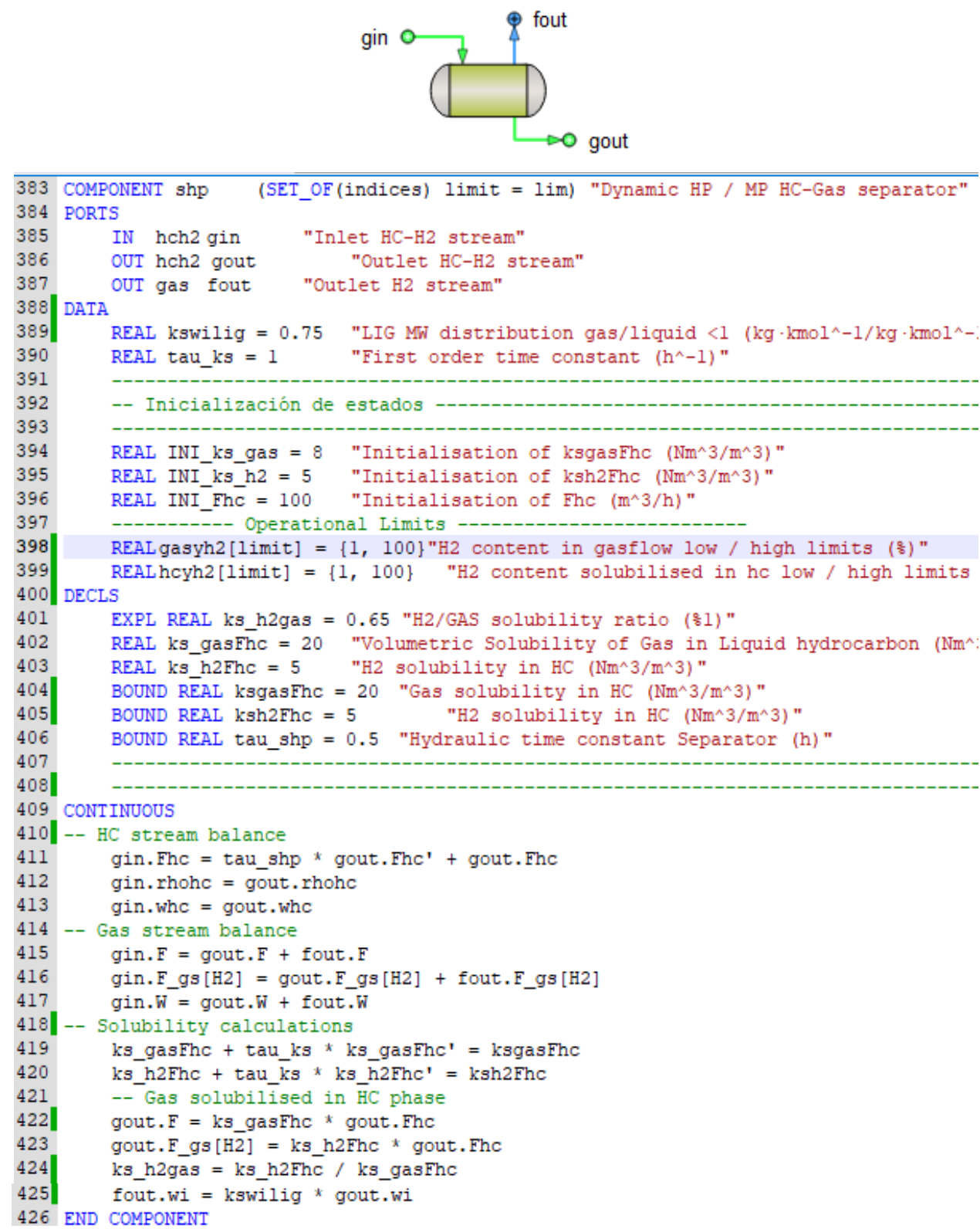

Fig. 2.15 - High and medium pressure separator unit (DoF = 6). 


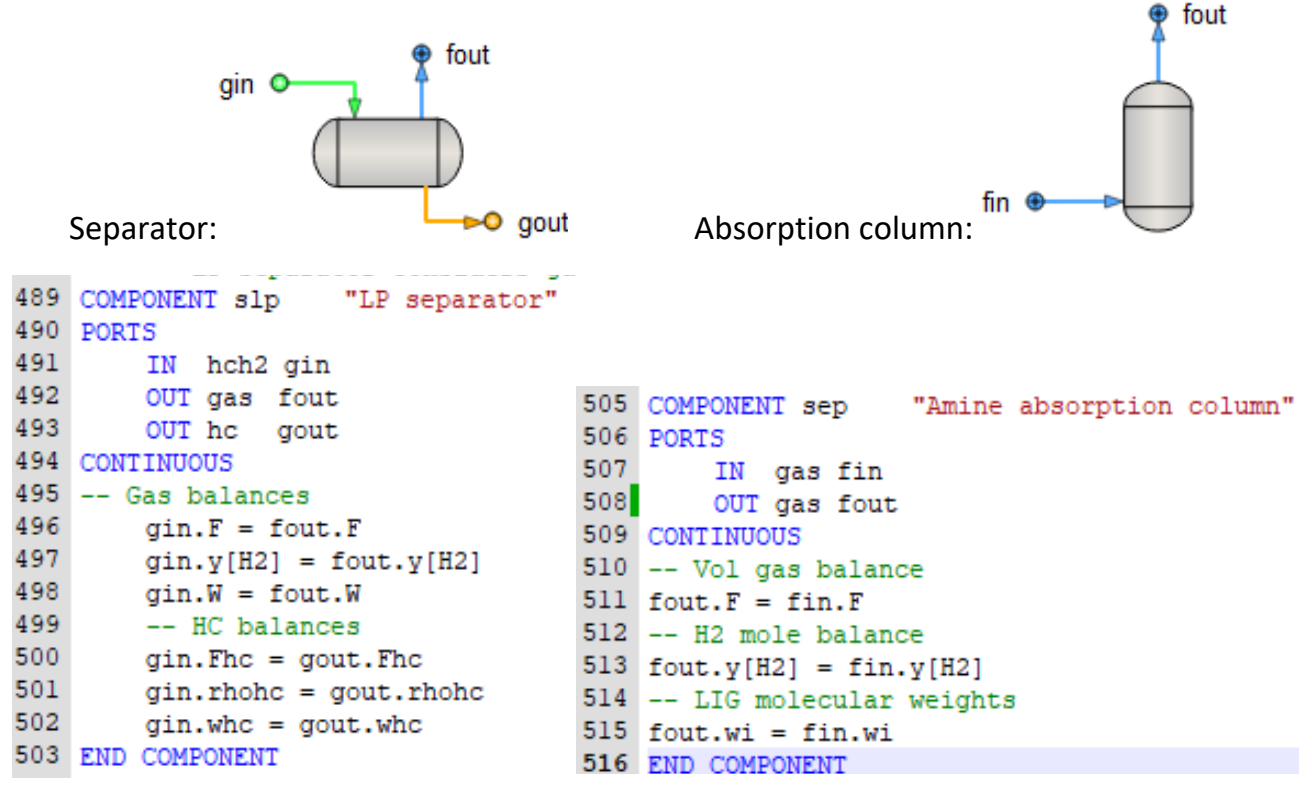

Fig. 2.16 - Left: Low pressure separator component (DoF =6). Right: Amine absorption column (DoF $=3)$.

Absorption columns are a relatively simple component from the modelling perspective, since no mass transfer takes place there. In a real plant the absorption columns carry out the hydrogen sulfide sweetening before the recycle gas reaches the recycle compressor. However this difference, the accuracy of the model is considered adequate. Nonetheless, a potential improvement for future research might well be the incorporation of multiple gas and liquid species. These should support detailed studied on how big an impact is on computational performance of a multiple species model compared with two-gas model.

Absorption columns (see code in Fig. 2.16-Right) have three degrees of freedom (DoF = 3), as simple pipeline connections.

\subsubsection{MISCELLANEOUS EQUIPMENT, INSTRUMENTATION AND CONTROL DEVICES}

This category is threefold:

- $\quad$ purification units (membranes, pressure swing adsorption), 
- compressors (reciprocating and centrifugal),

- flowmeters and flow controllers.

\subsection{PURIFICATION UNITS}

Purification units are of key importance in hydrogen systems, since they provide additional capability of hydrogen rich gas purification (typically from recycle streams) at almost no operational cost other than purged gas. Unfortunately, their internal mechanisms such as membrane diffusion and pressure adsorption are far from simple to be modelled based on first principles rigorously. Therefore, a so called grey-box approach is used to represent accurately enough how gas purity changes across the purification unit. These units have four degrees of freedom $(\mathrm{DoF}=4)$.

\subsection{Membrane purification units}

Membrane purification is a function of inlet stream purity (fin.y[H2]) and membrane purge flowrate (fpurg.F) over inlet stream flowrate (fin.F), as it is shown in Fig. 2.17 line 603 to 612. Moreover, an upper limit for permeate purity (Mem_model[HL]) and minimum purity (del_y_min) change help ensure a more realistic representation of the actual purification process. Parameters $a, b$, and $c$ are set to match experimental data properly. In this way, poor and good performance membranes only require their appropriate parameters. In essence, the membrane model is validated once the parameters are properly determined through test runs. Two examples of model validations with different purification performances are provided in further sections. 


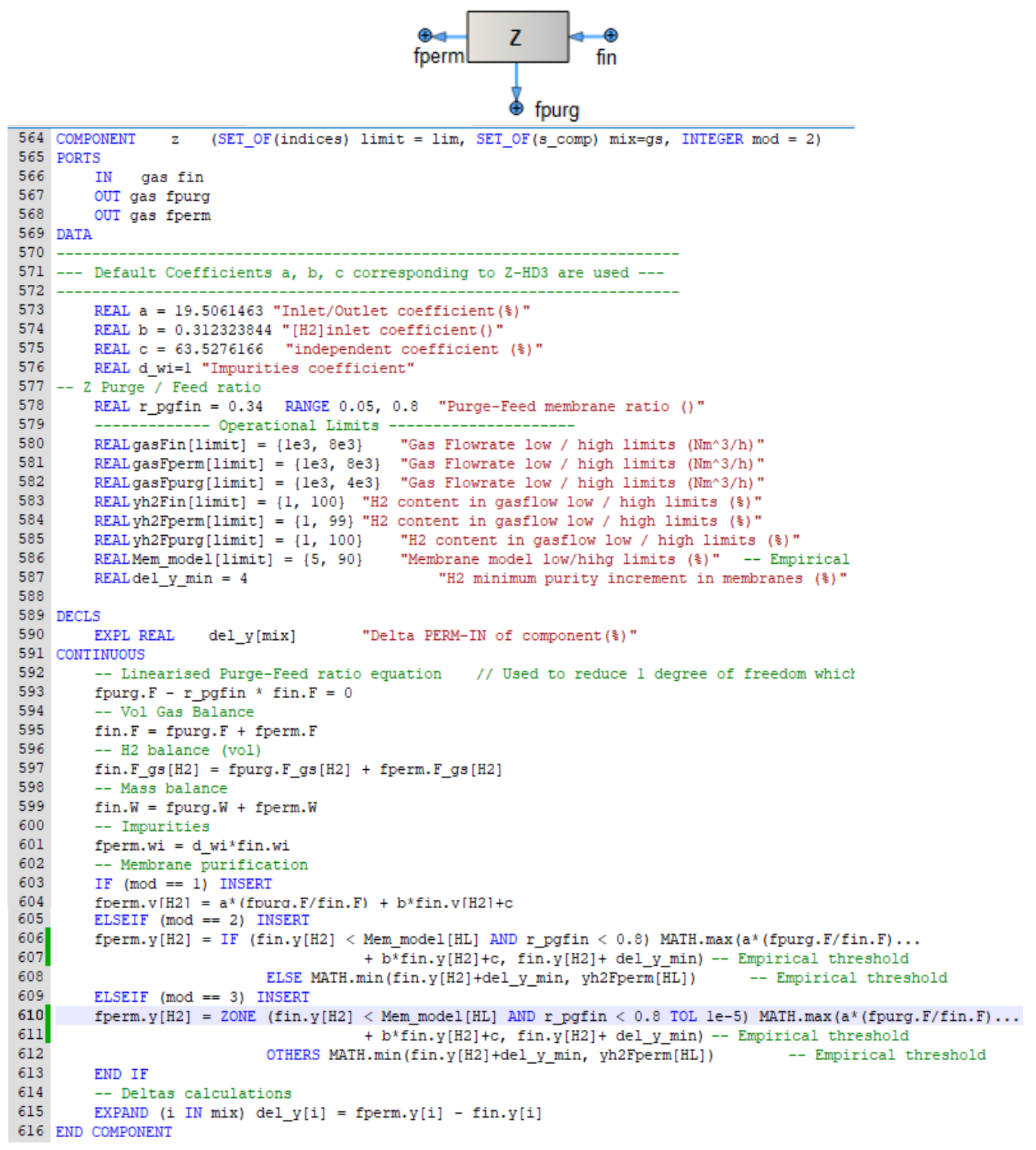

Fig. 2.17 - Purification membrane unit (DoF = 4).

\subsection{Pressure swing adsorption units}

Pressure swing adsorption (PSA) units, although more complex than membranes in their actual mechanism, are simpler to predict permeate purity (fperm.y[H2]) which is specified by the equipment supplier (etha). Therefore, at any allowable flowrate (fin.F) and inlet stream purity (fin.y[H2]), fperm.y[H2] remains unchanged (see Fig. 2.18, line 717 to 733$)$. 

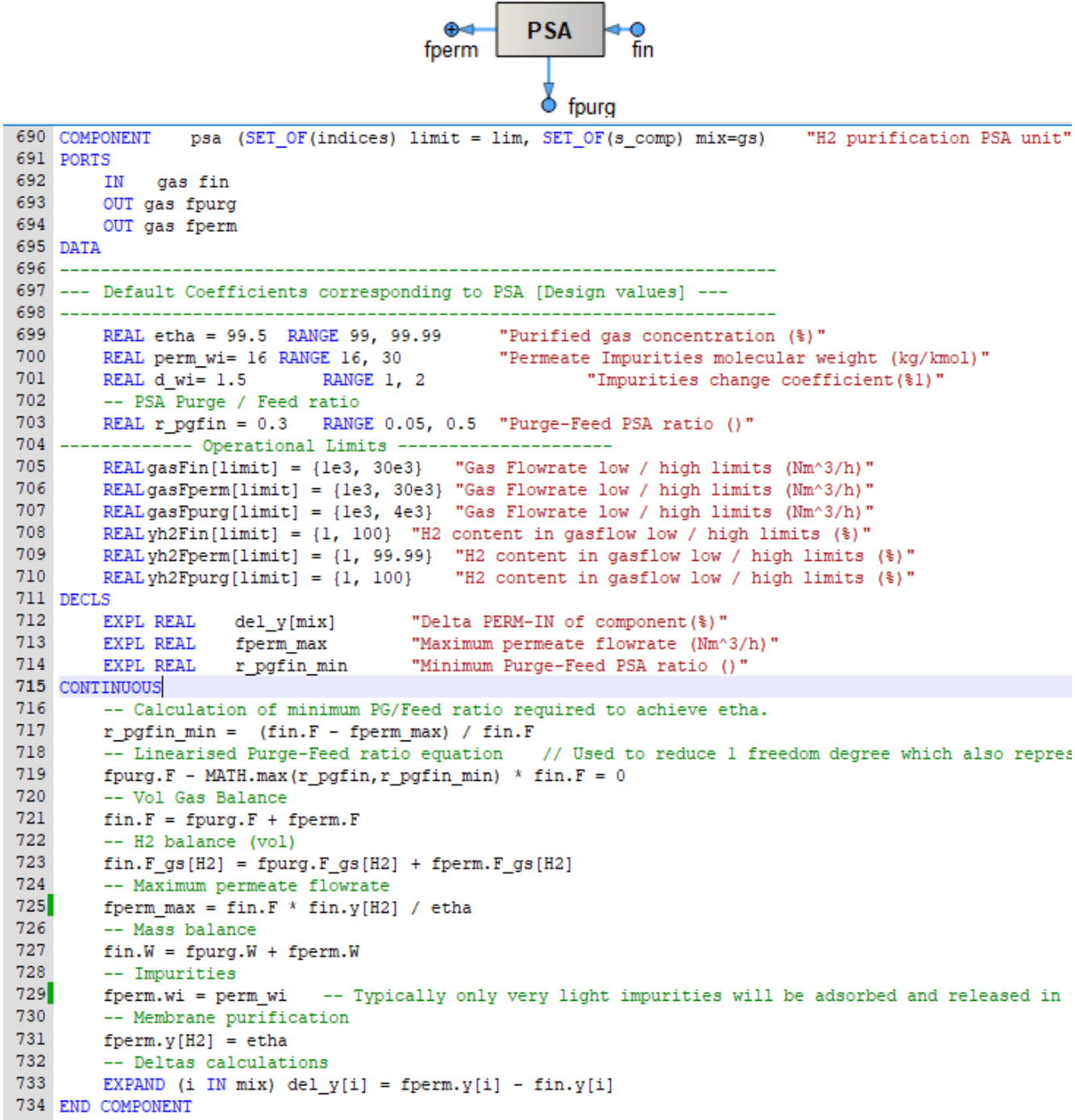

Fig. 2.18 - Pressure swing adsorption purification unit (DoF = 4).

\subsection{COMPRESSORS}

Compressors have proven dynamic behavior several orders faster than gases solubility and hydrogen demand. While compressor's changes are in the range of milliseconds up to a few seconds, hydrogen changes may take several minutes up to a few hours to realize, depending on the nature of the change. Therefore, are considered at steady state and their dynamic neglected. That is why, from an equation perspective reciprocating (Fig. 2.19, line 588 to line 590) and centrifugal compressors (Fig. 2.19, line 
601 to line 603) are identical, and are described by the same equation system as a simple pipeline (without pressure change).

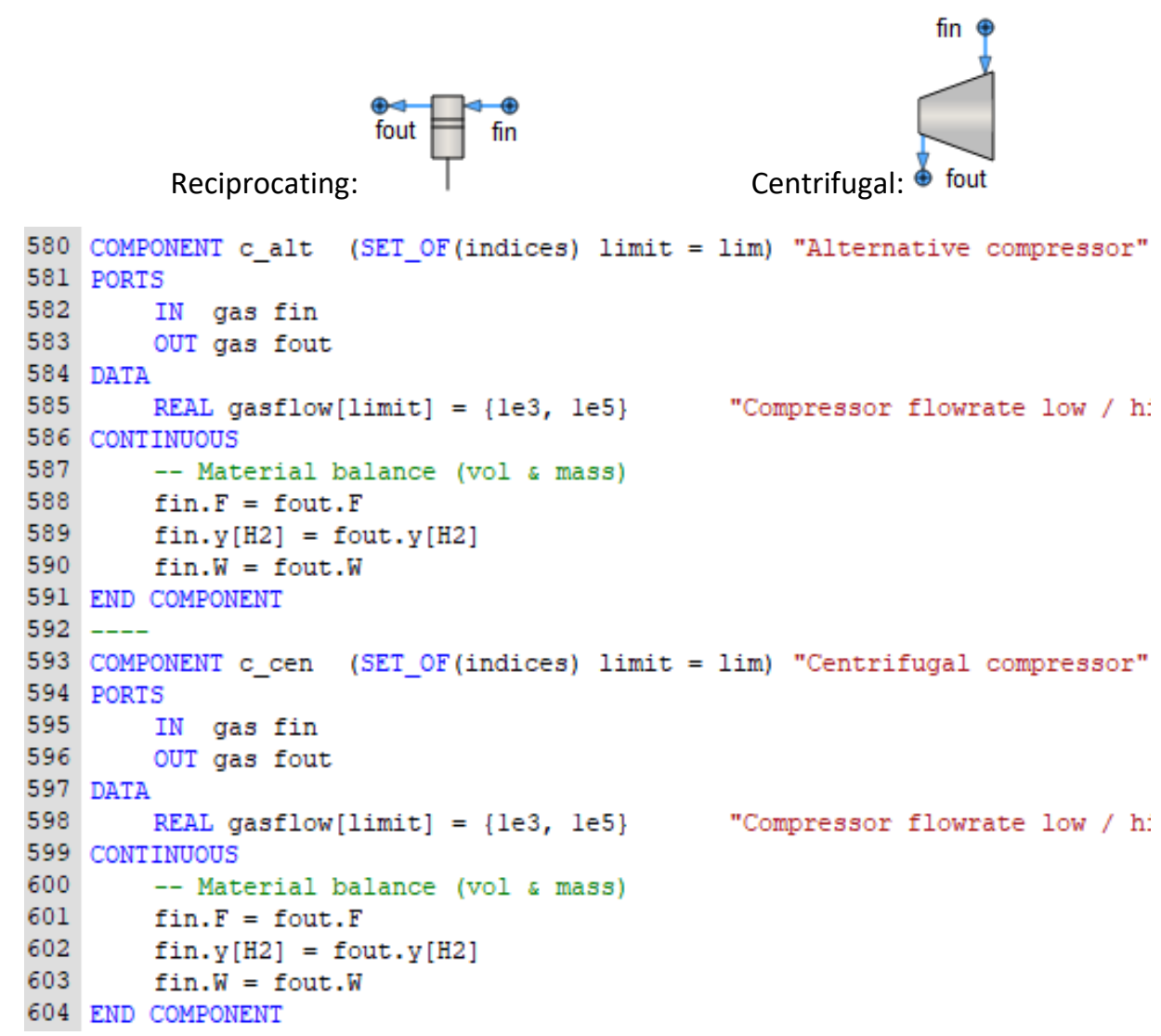

Fig. 2.19 - Reciprocating ("c_alt", line 580) and centrifugal ("c_cen", line 593) compressor components (DoF = 3).

\subsection{FLOWMETERS AND CONTROLLERS}

Flowmeters and flow controllers are both modelled as steady state equipment, based on the fact that their dynamic responses are negligible in the time frame of hydrogen demand and gas solubility changes in reactors and separators. The detailed code and equations is presented in Fig. 2.20. 


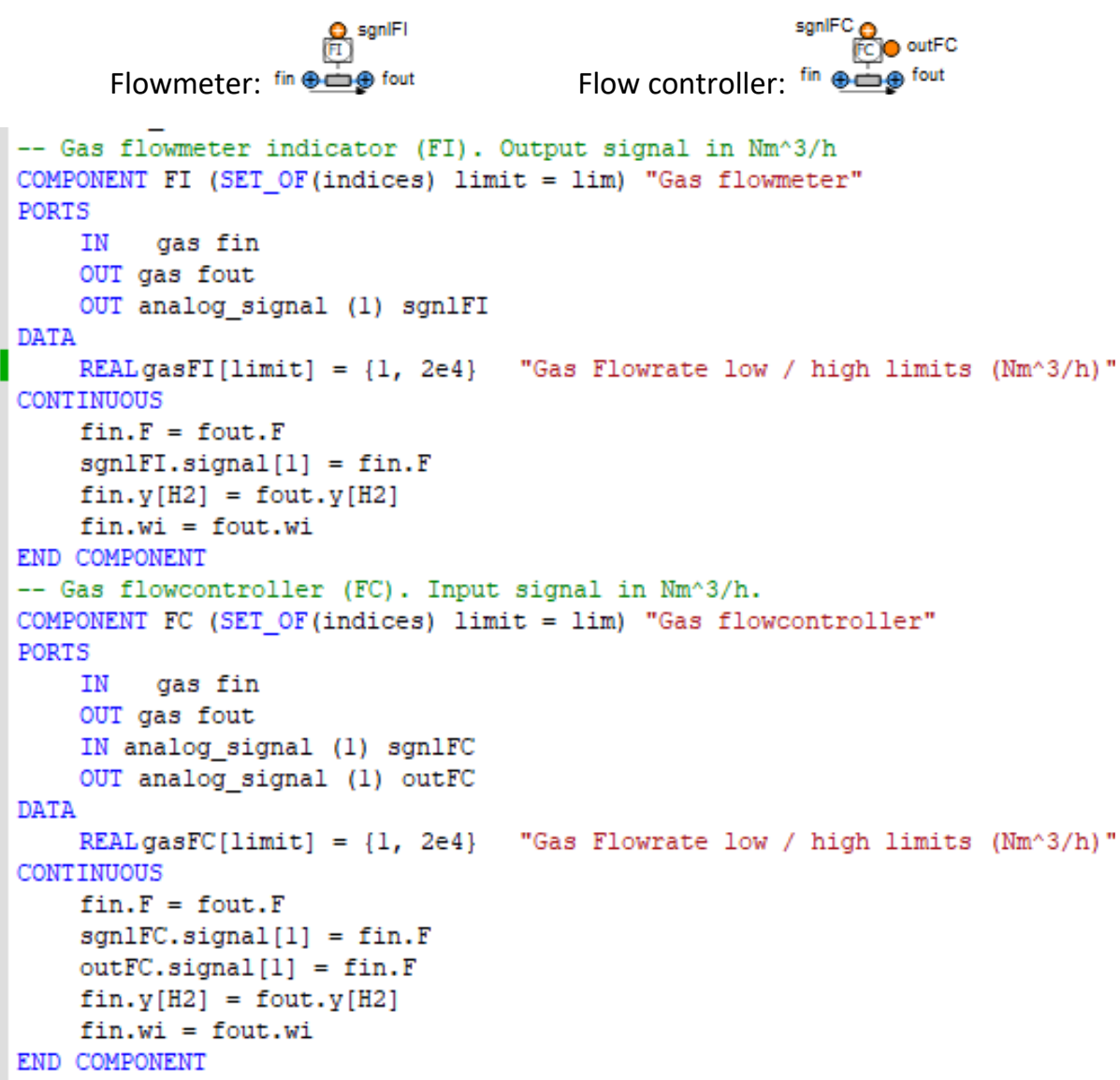

Fig. 2.20 - Gas flow meter $(F I$, line 12, DoF = 3) and flow controller $(F C$, line 26, DoF = 3) components.

\subsection{LIBRARY UTILIZATION AND VALIDATION PROCESS}

This library of refinery hydrogen network process units is suitable for underpinning complex decision support applications of large scale process systems. In practice, it enables a simplified formulation of hydrotreating process plants and their hydrogen network, which by process simulation can itself be of great value as a decision-making tool at many levels in process plants. For instance, process engineering staff would use a model for assessing plant performance under changing feedstock conditions, while the same model could be used by planning staff to study the impact on the hydrogen network of production campaigns, and so on. However, the main impact would realize when 
combined with state estimation techniques, along with online data collection methods. This idea is developed further and discussed in detail when presenting Real-Time Reconciled Simulation in the next chapter.

In addition, validation of the models against process data is required prior to going forward in their utilization in decision-making. In this respect, I spent a significant amount of time at Petronor refinery (Petronor, 2020) focused on model validation activities, see Appendix D. In fact, based on this dynamic library the whole refinery hydrogen network of Petronor (Petronor, 2020) has been modelled and simulated.

\subsubsection{Notes on Petronor hydrogen network library}

In practise, process unit models based on the dynamic simulation library are further compiled as components of Petronor's refinery hydrogen network library. This library consists of:

- fourteen hydrogen consumer process units,

- two high purity hydrogen producer process units (steam reformer units),

- two low purity hydrogen producer process units (platformer units),

- multiple interconnecting and supply headers (e.g. low purity header, unit trains headers, etc.).

It is noteworthy that, none of the above mentioned components of the library is a mirror image of one another, which is typically the case when addressing actual industrial complexes that suffered multiple transformations over the years. In other words, the modeling effort faced has been significant as it required meticulous surveying of plant documentation, including process and instrumentation diagrams (P\&IDs), operating manuals, among other sources. I gratefully acknowledge that, a first pass of this surveying and modeling effort was done by (de Prada et al., 2017; Gómez Sayalero, 2016), these works served as valuable references to this process unit library, as well. 
In terms of models validation process against plant data, this library has been developed mirroring reconciled steady state data from a prototype real time optimization, already implemented in collaboration with Petronor in previous research efforts (de Prada et al., 2017; de Prada and Sarabia, 2018; Sarabia et al., 2012). Moreover, for practical reasons variables resulting from the reconciliation and optimization were compared next to each other with plant data in tailored made historian schematics (see Fig. 2.21 as an example schematic), which were critical in validating steady state models and therefore its variables.

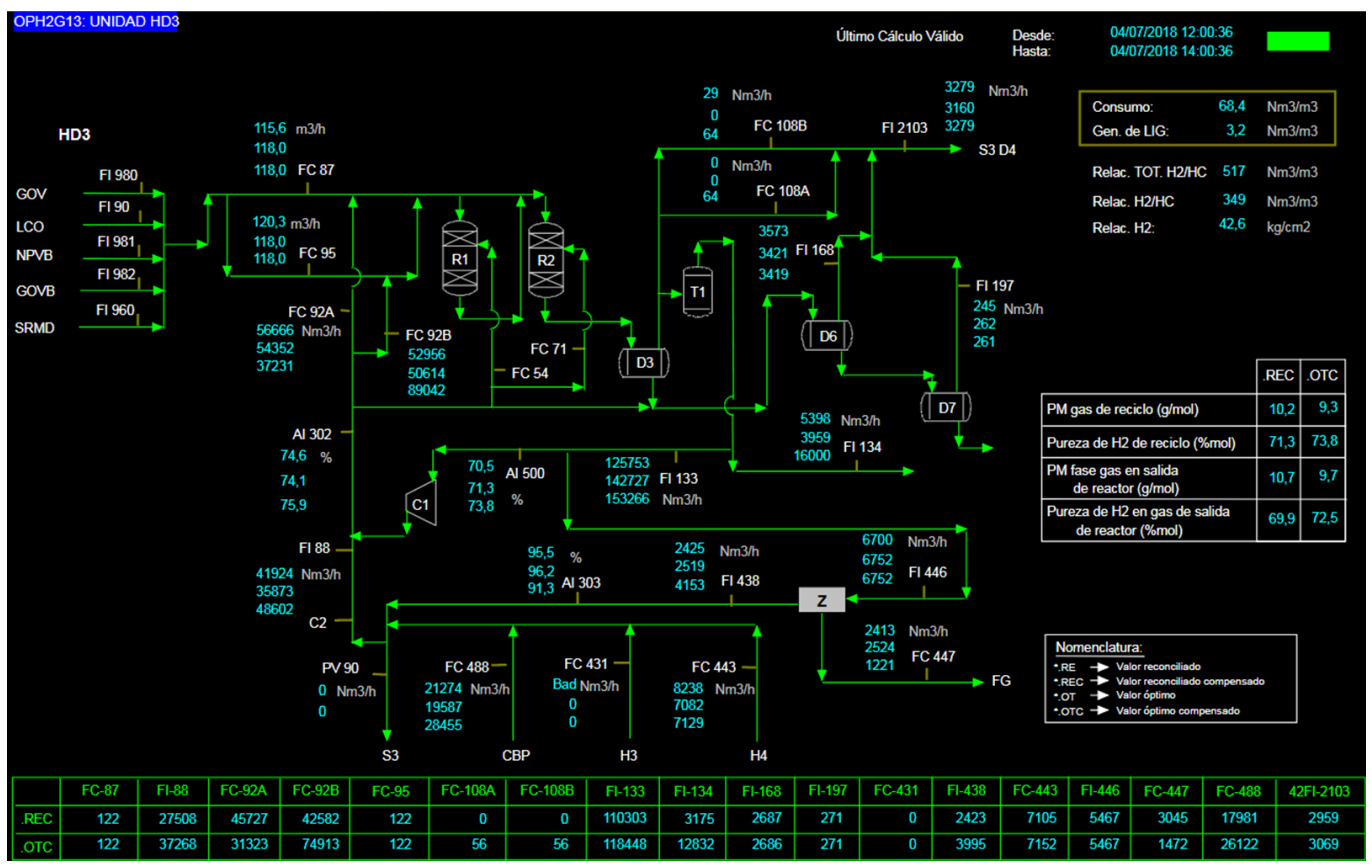

Fig. 2.21 - Schematic of unit G3 showing average plant data, reconciled data and optimal hydrogen distribution data. Next to each instrument tag, the top value corresponds to plant average readings, the middle is the reconciled value and the bottom values is the value if hydrogen distribution is optimized.

This qualitative process was conducted alongside Petronor staff, control and process engineers, helping in a prompt acceptance of most of the findings resulting from this validation. In addition, dynamic behavior has been qualitatively validated against plant data as well as in technical review meetings with Petronor staff, which added practical insights during modeling and validation stages. In Appendix $D$ is provided a summary of 
the significance of these technical reviews in shaping this work towards something useful for decision making in process operation environments.

In actuality, components for each process unit and header in the network are compiled and linked to their tailored-made palette of icons (see Fig. 2.22 and Fig. 2.23). Thereafter, these icons are used to properly develop the network model by connecting units and headers together using drag-and-drop feature of EcosimPro ${ }^{\circledR} /$ Proosis $^{\circledR}$ (EA Internacional, 2019a) onto the hydrogen network-wide schematic (see Fig. 2.24).

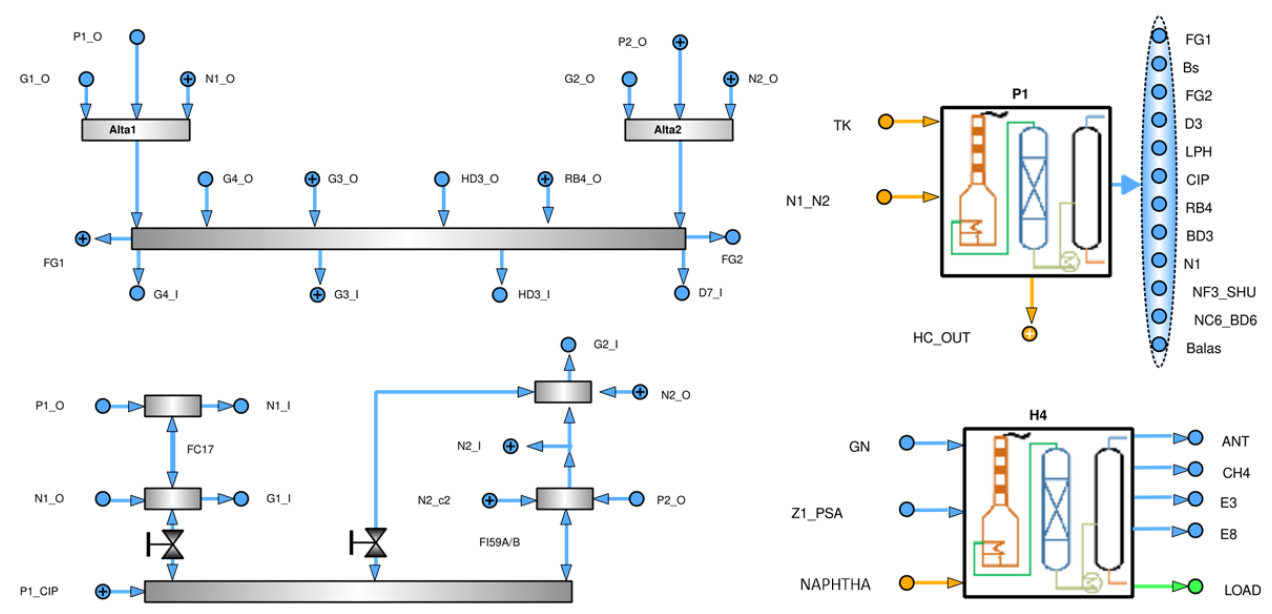

Fig. 2.22 - Examples of headers (in grey) and hydrogen production units (P1 and H4). Orange circles show liquid hydrocarbon streams. Blue circles show gas streams. Green streams show mixed gas and liquid streams.

It should be acknowledged that, this network-wide dynamic simulation is continuously under review with respect to its validation against plant data and decision support usefulness, which will certainly lead to a reviewed version of the dynamic simulation library as part its continuous improvement. For instance, the large presence of algebraic loops in the network-wide model has been identified as a challenge that required attention. In particular, the incorporation of fast dynamics in some variables has proven to be effective in minimizing the presence of algebraic loops in the final mathematical model improving its solvability in most cases. However, these minor changes are currently being tested and undergoing validation, hence, not described 
further in this thesis as they are not finalized yet. Currently, the network simulation has been successfully validated against a set of reconciled plant data.

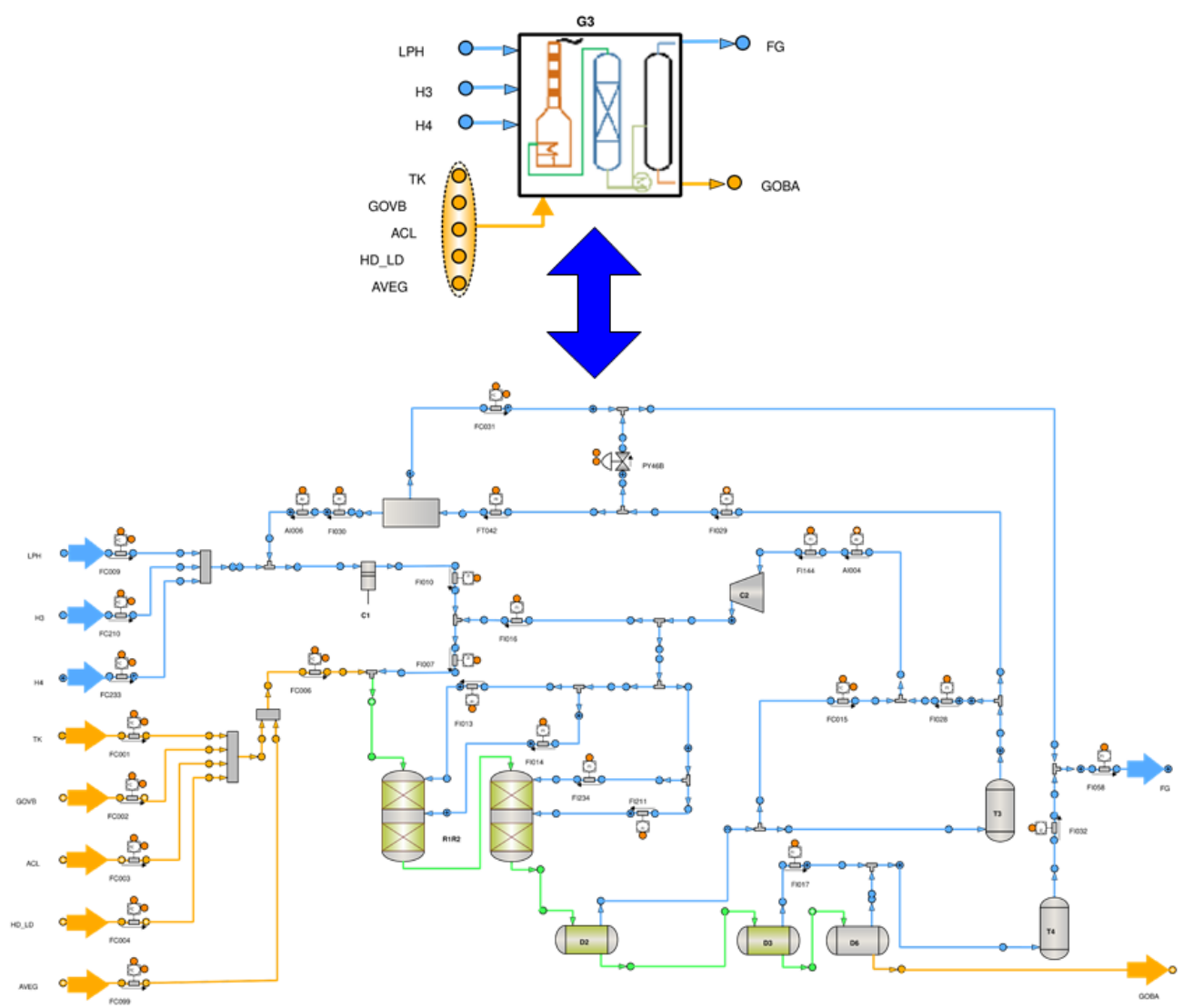

Fig. 2.23 - Example of visual representation of process unit G3 compiled into one component and its icon. 


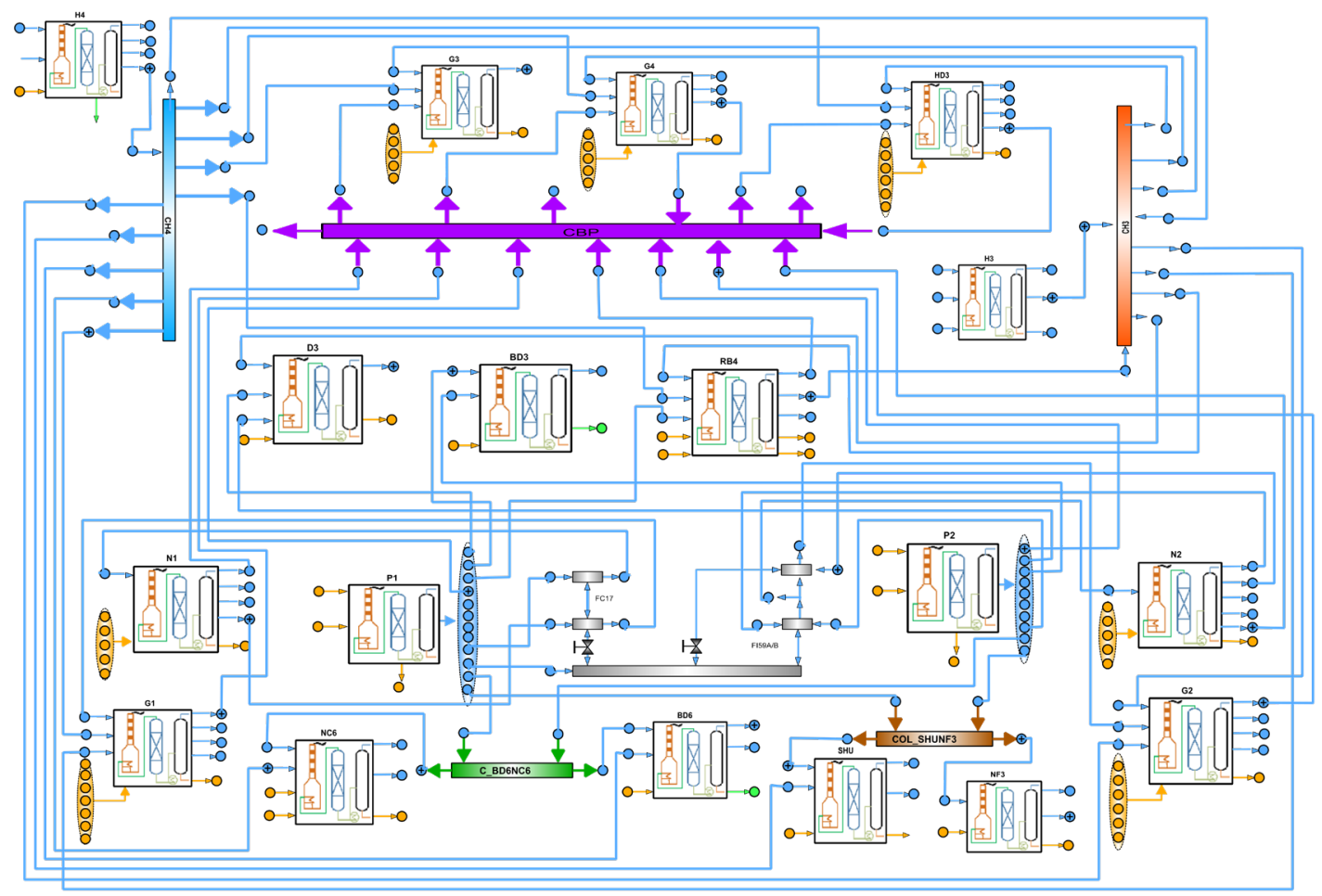

Fig. 2.24 - Schematic of the refinery hydrogen network simulation model.

As an example and in the interest of being brief, in the next section I describe the validation process of purification membranes, which are instrumental in the optimal management of the refinery hydrogen network as it has been documented by Galan et al., (2018).

\subsubsection{MEMBRANE MODEL VALIDATION}

Grey-box models, such as the ones utilized for membrane units require sensible validation against data in order to be of use. For this purpose, test runs with focus on purification performance are to be carried out, along with sensible process data collection. In particular, this model has been validated on two membrane (ZHD3 and ZG3) units of Petronor refinery (Petronor, 2020) in Muskiz, Spain. Further usage of the validated model supported other research work and hydrogen management policies, most of these are presented in detail by Galan et al. (2018). 
Table 2.2 shows the parameters $a, b$ and c for membranes ZHD3 and ZG3 based on data of on-stream periods in years 2016 to 2017. Due to the particularities of each purification unit, ZHD3 parameter estimation is based on 9541 data points, while ZG3 is based on less than 30 data points. This difference is explained by the fact that ZHD3 has an online analyzer, which measures hydrogen concentration at both ends of the membrane. In both cases, a two days test run was conducted for specific purified gas analysis in the plant laboratory.

Table 2.2 - Membranes ZHD3 and ZG3 parameters based on 2016 to 2017 operation campaigns applying conventional minimum square root method.

\begin{tabular}{|l|l|l|l|l|}
\hline & Units & Description & ZHD3 & ZG3 \\
\hline a & $\% \mathrm{H}_{2}$ & Purge-feed ratio coefficient & 19.5061 & 7.4933 \\
\hline b & - & Feed purity coefficient & 0.3123 & 4.1041 \\
\hline c & $\% \mathrm{H}_{2}$ & Independent coefficient & 63.5276 & -307.6310 \\
\hline
\end{tabular}

In light of the performance shown by both membranes, which is reflected by parameters $a, b$ and $c$, it is important to note that ZHD3 increases hydrogen purity several times more than ZG3. This is mostly explained by its larger $a$, as well as $c$, compared with ZG3. In addition, membranes have to run at almost fixed purge to feed ratios (around 0.34), due to operational restrictions. Fig. 2.25 shows ZHD3 and ZG3 performance estimations based on the validated model for a typical range of feed purities and at 0.34 purge-feed flowrate ratio (r_pgfin in Fig. 2.25 line 578). In effect, ZHD3 outperforms ZG3 at all purities considered, though ZG3 improves considerably its purification performance at high feed purities. Another way of assessing the performance of these units is simply looking at its purity increase across the membrane, where ZHD3 ranges between $10 \%$ to $18 \%$ and ZG3 is less than $7 \%$ at its best performance. These findings were corroborated with plant historian data when available. A comparison chart is presented in Fig. 2.26 where, online analyzer data of ZHD3 is plotted alongside estimations to show its accuracy. It should be appreciated, that due to multiple factors this piece of equipment is operated by the field operator manually in coordination with the control room operator. In addition, the hydrogen analyzer has proven poor performance, see Fig. 2.26, in the long run, which 
can be attested by its erratic behavior. Therefore, the accuracy validated with the estimation is considered enough.

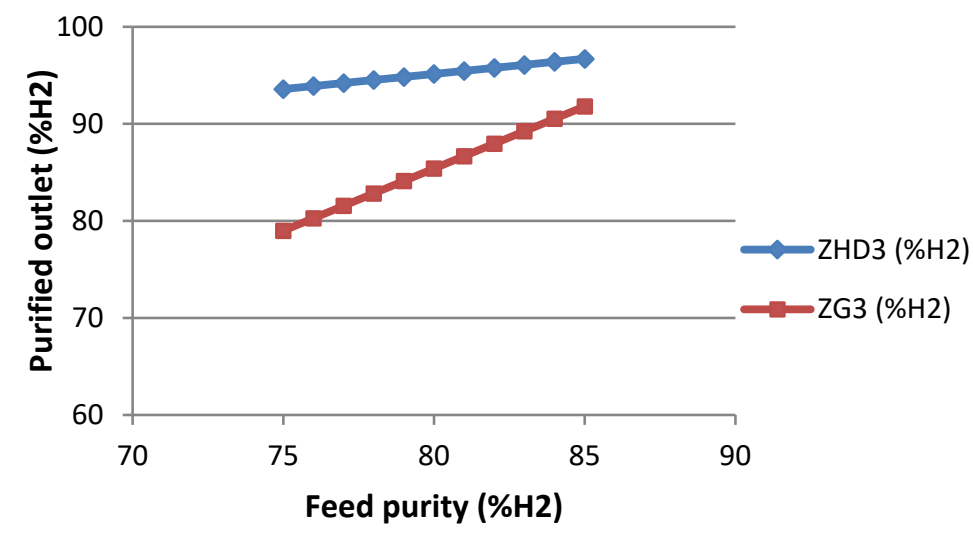

Fig. 2.25 - ZHD3 and ZG3 purified outlet stream estimations applying validated coefficients when considering typical feed purities and purge-feed flowrate ratio at 0.34 .

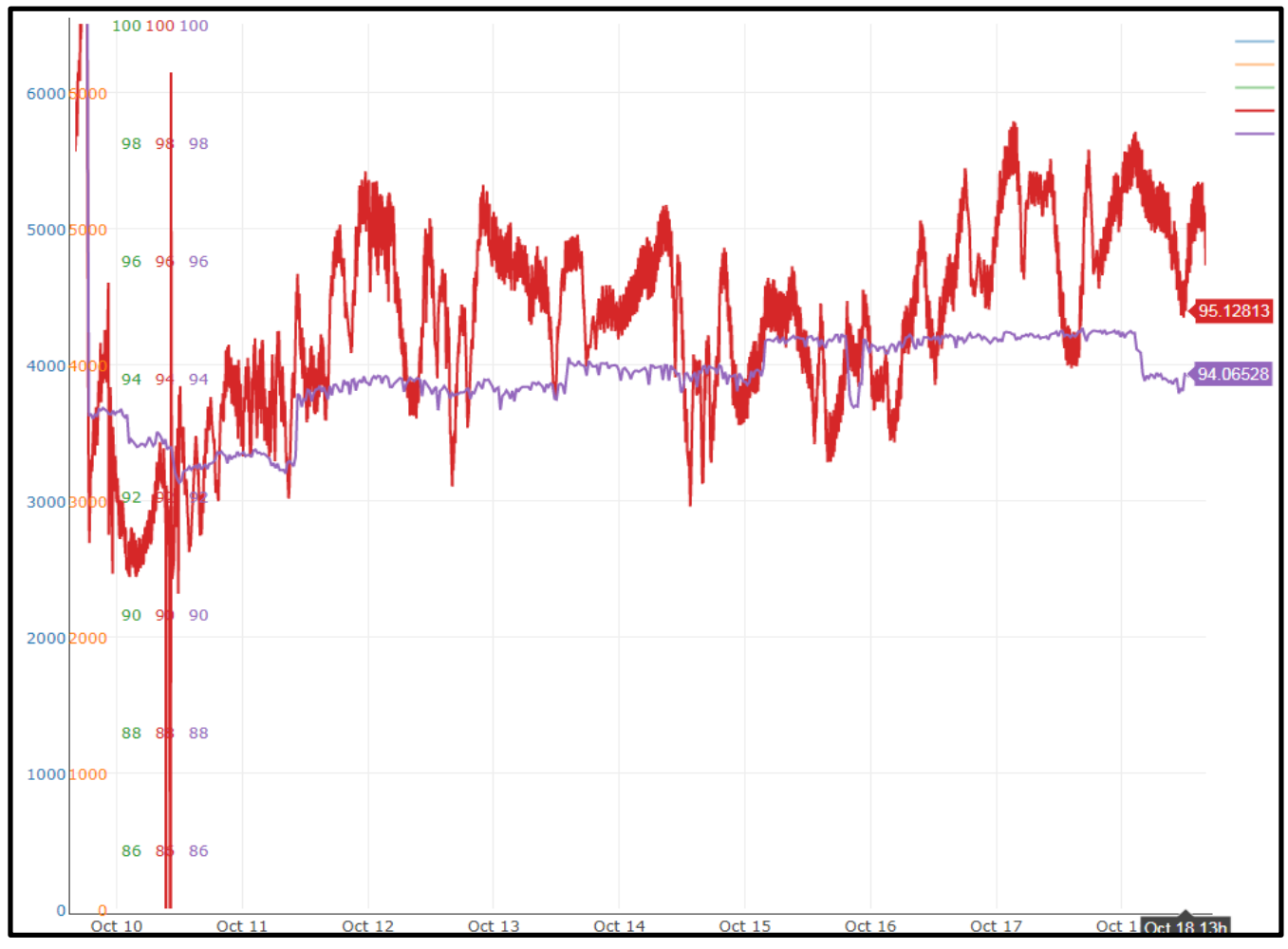

Fig. 2.26 - Online analysis of ZHD3 in red, and estimated values in purple between 10-Oct-2017 and 18-Oct-2017. 


\subsubsection{SIMULATION AIMED FOR PROCESS DESIGN}

Another and more straightforward utilization of this library is in hydrogen network design, by building different network arrangements and simulating their behavior. Although there are commercial software available capable of this (Hysys, PetroSim, etc.), these require significant amounts of time to set large dynamic systems. Basically, these are data intensive with broad scope, rather than specific and focused as is the case of the hydrogen network library. Consequently, dynamic simulation is typically applied in industrial case studies of limited scope only where absolutely necessary. In this sense, a simplified rather than rigorous first principle model would be of use, bridging the gap between process design simulation and operation. If it is used wisely, one would be able to develop a plant model that sits somewhere in between, rigorous dynamic model and steady-state model.

\subsubsection{ENHANCED SIMULATION FRAMEWORK: AN EXAMPLE}

Plain simulation capabilities are enhanced when the mathematical model exchanges information with other environments while it is executed. This sort of application is called as an enhanced simulation framework in the context of this thesis. Enhanced simulation frameworks, feature an efficient communication system with other applications within the framework, while bringing a mathematical model aimed at aiding in the decision-making process. In essence, combines in the same framework process data collection, basic logic decisions, with dynamic simulation. An example of such an enhanced simulation is a stand-alone application based on the dynamic simulation library, and programmed for being executed on Excel ${ }^{\circledR}$. The model used is presented in detail in the following chapters. A flow diagram showing the main information flow and architecture of the application is presented in Fig. 2.26. It is important to highlight the fact that most commercially available historians in the process industry are fairly efficient in their communication functions with Excel, which is the main reason behind the decision of developing this case study on an Excel stand-alone framework. The model in question corresponds to plant G3 in Petronor refinery, which has been used as a case study across 
this thesis at many stages. Figure 2.27 presents an example of how G3 model may be integrated into Excel $^{\circledR}$, so a simulation can be executed and manipulated from there.

It should be pointed out that, EcosimPro ${ }^{\circledR}$ / PROOSIS ${ }^{\circledR}$ (EA Internacional, 2019a) does provide a feature for automatic generation of executable files that can be called from Excel. However, there are several tasks that should be carried out individually on a manual manner by the developer of the application. Some examples of these are: configuration of the executable file, including setting accessible variables from outside EcosimPro ${ }^{\circledR}$ / PROOSIS $^{\circledR}$ (EA Internacional, 2019a), pulling data from plant historian and any logics required to run the simulation successfully. In part, G3 serves as example of a successful integration of all the aforementioned, performed by someone with programming skills at basic level only. Needless to say that, other alternatives to Excel $^{\circledR}$ may be equivalent at the time of calling plant data, and executing a simulation using a compiled model based on the library presented. An analysis of those alternatives and their performance is out of the scope of this research.

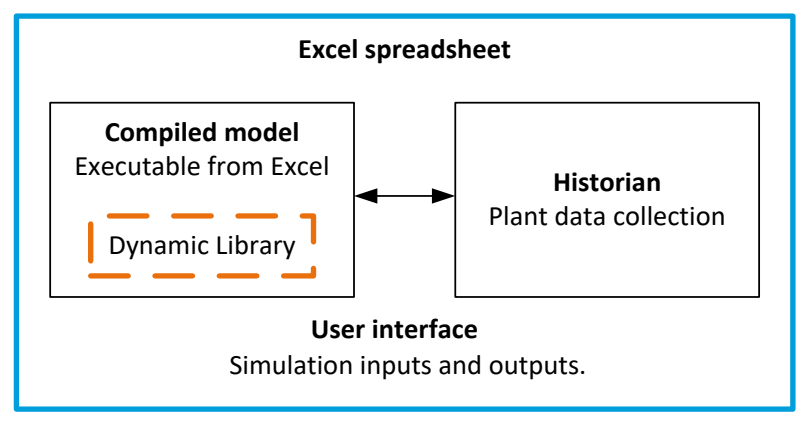

Fig. 2.26 -An example of an enhance simulation framework information flow and architecture as a stand-alone simulation application. 


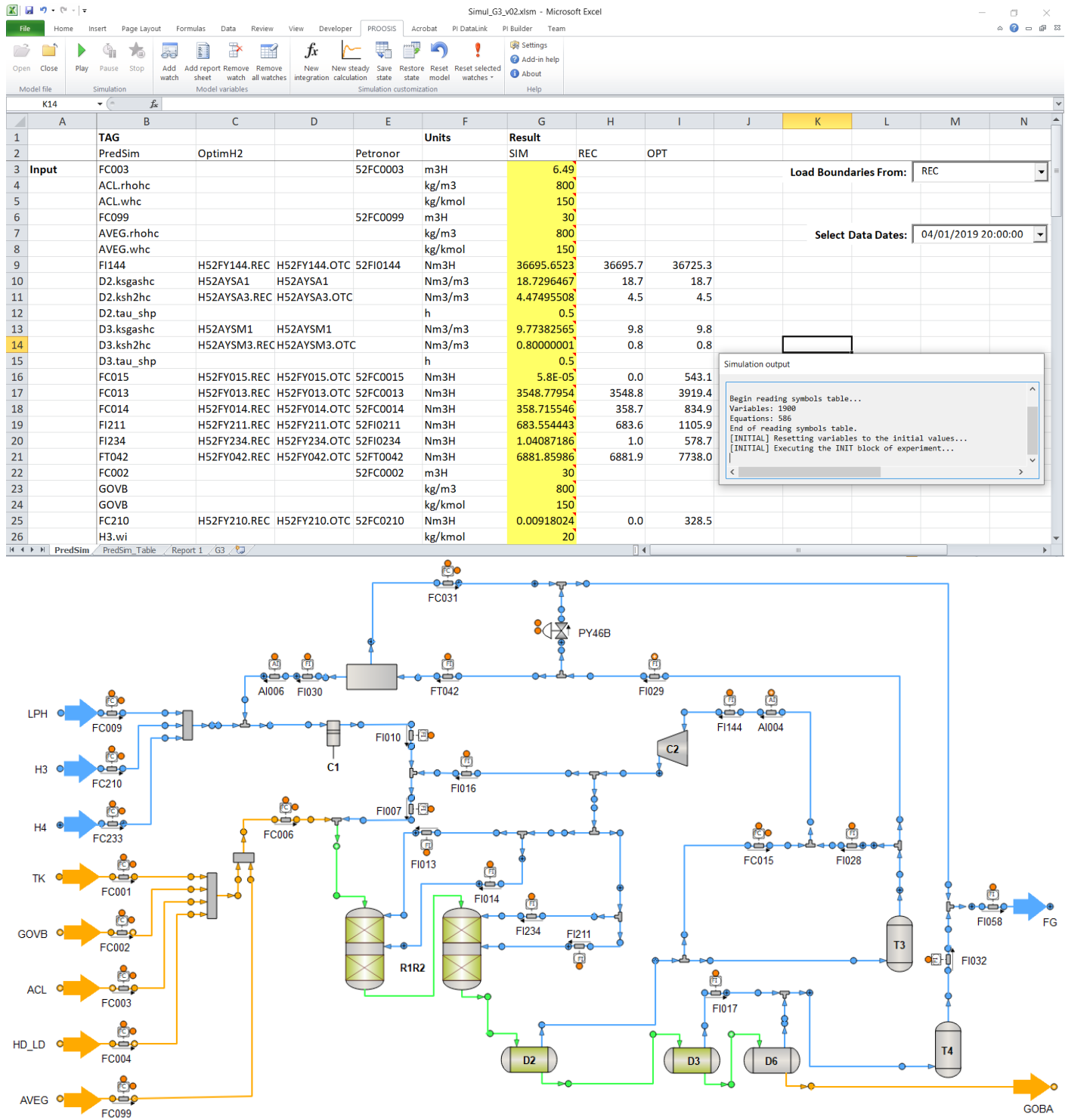

Fig. 2.27 - G3 stand-alone simulation embedded into Excel, as an example of utilization of the dynamic simulation library.

\subsubsection{DYNAMIC SIMULATION UTILIZATION IN REAL-TIME ENVIRONMENTS}

In order for real-time simulation (RTS) tools to accomplish actual representation of up-to-date plant conditions, simulations should be efficiently fed with online data, using accurate and consistent models. Furthermore, dynamic libraries become critical in this workflow as these support the fundamental mathematical models underpinning online 
and offline simulations. In particular, these goals require specific architectures to enhance information and data exchange amongst different tools, in what I name decision support framework (DSF). Although DSF are addressed in chapter 5, it is important to showcase the role of the dynamic simulation library in this context (see the red box in Fig. 2.28).

Online process data is central to the proposed architecture of DSF as it is represented in the simplified diagram in Fig. 2.28. That is why, the historian database, which collects and manages online data from several sources is at the center of the architecture. The historian provides input data and equipment information to the realtime reconciled simulation (RTRS) block, which performs state estimation routines by means of the moving horizon estimation (MHE) module and executes real-time simulation using the dynamic simulation library. These results can be presented directly by the simulation interface, even aided by spreadsheet tools in a standalone manner (as discussed in the previous section 2.5.3), or sent to the historian for ease of management and pulled from another interface. Either way, the simulation results should be easily accessible to decision-making stakeholders and end users at various levels, from control room operators (CRO) to managers and engineers. Actually, one of the main challenges to overcome is to provide information with the right level of detail and complexity to the appropriate user, appreciating that not all decision makers require the same amount of information, even if it is well presented. Typically, on one end operators should be aided with specific operational information, therefore simulation outputs would highlight variables that operators are familiar with instead of chemical properties. On the other end, process engineering staff may be interested in detailed compositions and estimations of stream properties. 


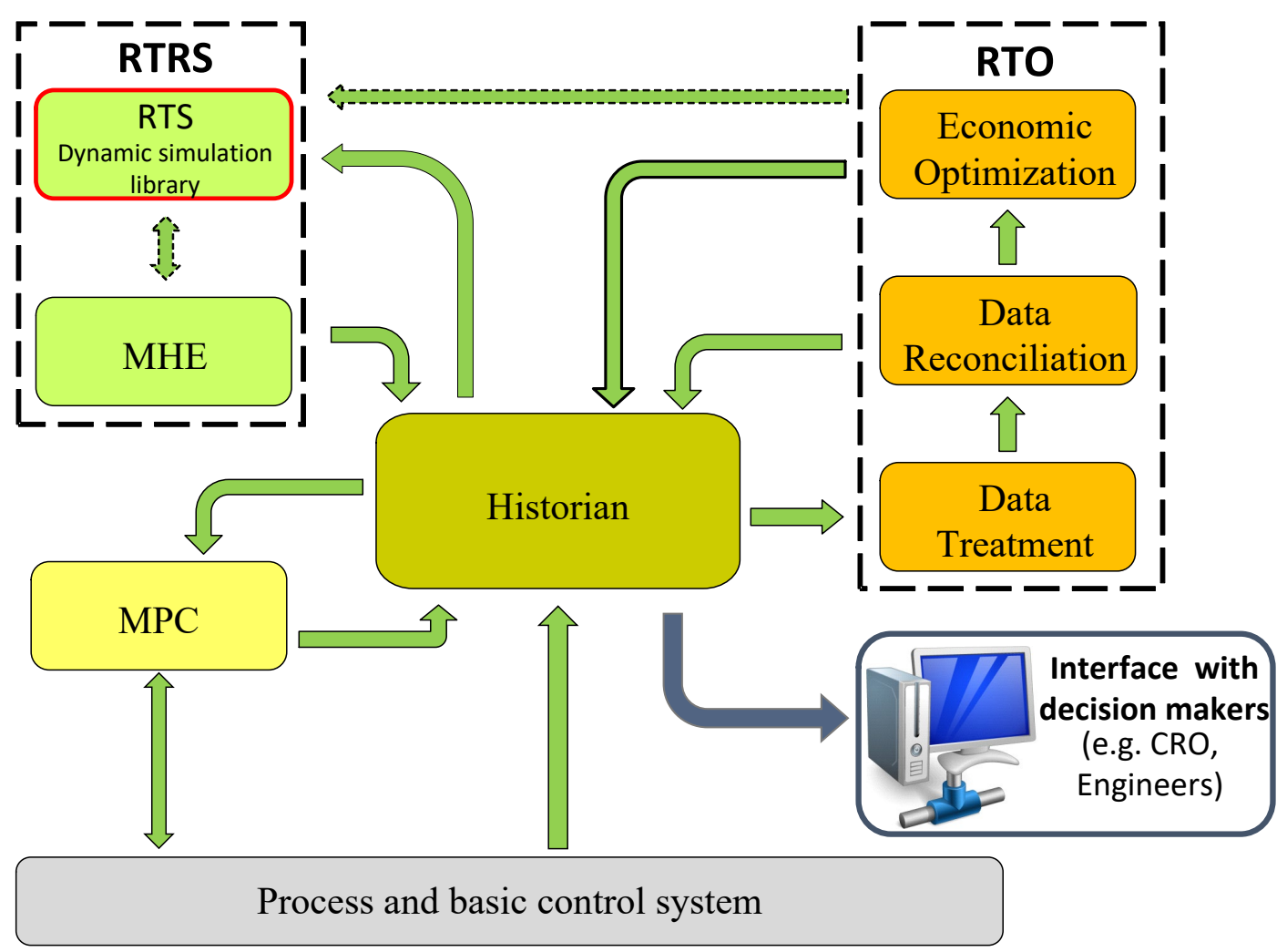

Fig. 2.28 - Simplified architecture of decision support framework. In red the real-time simulation (RTS) module, supported by the dynamic simulation library. RTRS: real-time reconciled simulation. MHE: moving horizon estimation. MPC: model predictive control. RTO: real-time optimization. CRO: control room operators. Dashed arrows denote optional information flows.

\subsection{CONCLUSIONS}

A dynamic simulation library aimed at supporting plant wide decision making is developed and presented. This library is based on simplified first principle models applied on several process equipment that play a key role in refinery hydrogen networks. A detailed description of the most important components and their mathematical models is discussed. The process equipment list consists of reactors, separators, purification units, mixer and splitters, amongst others. Furthermore, an introductory explanation of its purpose in a hydrogen network context is provided. For this purpose, a network-wide hydrogen network model case study is briefly discussed, with focus on how the main 
dynamic simulation library is used as the backbone of higher level library of process units used for network-wide simulation.

In addition, a case study of model validation of membrane purification units is briefly presented and discussed. The validated model is presented against online measured data to its in light of its key role in hydrogen management.

Complex process arrangements can be efficiently represented and translated into a mathematical model for running a simulation alone or further interaction with other computer-based tools.

A very unique feature of this library is its potential for supporting stand-alone simulation models, which can be fed from other sources in a semi-automatic manner. This utilization of the library is called enhanced simulation framework, and a case study of a real process plant in a hydrogen network is introduced as a demonstration. 
3 REAL-TIME RECONCILED

SIMULATION 



\section{Abstract}

Decision support tools in the process industry have been gaining relevance, especially for operation under uncertain conditions. This study describes real-time reconciled simulation, and analyzes its usefulness as decision-making tool for process operators, especially under unexpected process changes. The proposed methodology is implemented in two case studies in the context of an oil refinery hydrogen network, both plant and network levels are considered. A what-if analysis is conducted on case studies, assessing two feasible mitigation actions for each case baseline condition. The focus of the discussion is, nevertheless, on the methodology itself and its general features as decision support tool. In terms of mitigation actions, these are assessed with regards of its economic impact on the system in question. It is shown how actions at plant level may be disadvantageous when facing hydrogen demand changes, compared to network-wide mitigation actions. At plant level, it is pointed out the importance of purification units, prevailing over hydrogen make-up for mitigation of demand change. It is highlighted the fact that RTRS complements in a straightforward manner other control operation tools such as model predictive controllers (MPC) and real-time optimizers (RTO). Therefore, it may add to any decision support framework an open-loop component with parameter estimation and forecasting capabilities. Moreover, its potential for training and integration within other tools packages is discussed. Future directions of research are commented such as fully integrated decision support frameworks, including RTRS, MPC and RTO. 



\subsection{INTRODUCTION}

In the context of this thesis, real-time reconciled simulation (RTRS) is defined as a system simulation, in which all current parameters and states (measured and unmeasured) arise as a result of a DR based on past and current plant data. Moreover, is capable of representing the future condition of the system within a simulation time horizon, provided that certain boundaries or manipulated variables (MV) are given.

From its definition, RTRS can be seen as a real-time simulation tool which incorporates parameters and states estimations based on past data from the system. Fig 3.1 shows an information flow schematic of a decision support framework (DSF) with a RTRS application. These capabilities motivate the interest in developing further RTRS applications. In particular, its combination of open-loop decision support and plant forecasting features are the most outstanding takeaways of these tools. Moreover, it is key for RTRS usefulness an effective integration of forecasting features along with current plant estimation status and ease of utilization by end-users, e.g. control room operators (CRO), plant engineers. Furthermore, from a practical point of view, RTRS should exchange information with other decision-making applications within the DSF such as estimated parameters from the RTO, which can be useful starting points of the state estimation or simply used as baselines when exercising different operating sets of actions as in What-if analysis assessments. I get deeper into the details of the architecture of RTRS in further in the text, and focus on the proposed workflow of the DSF in chapter 5. 


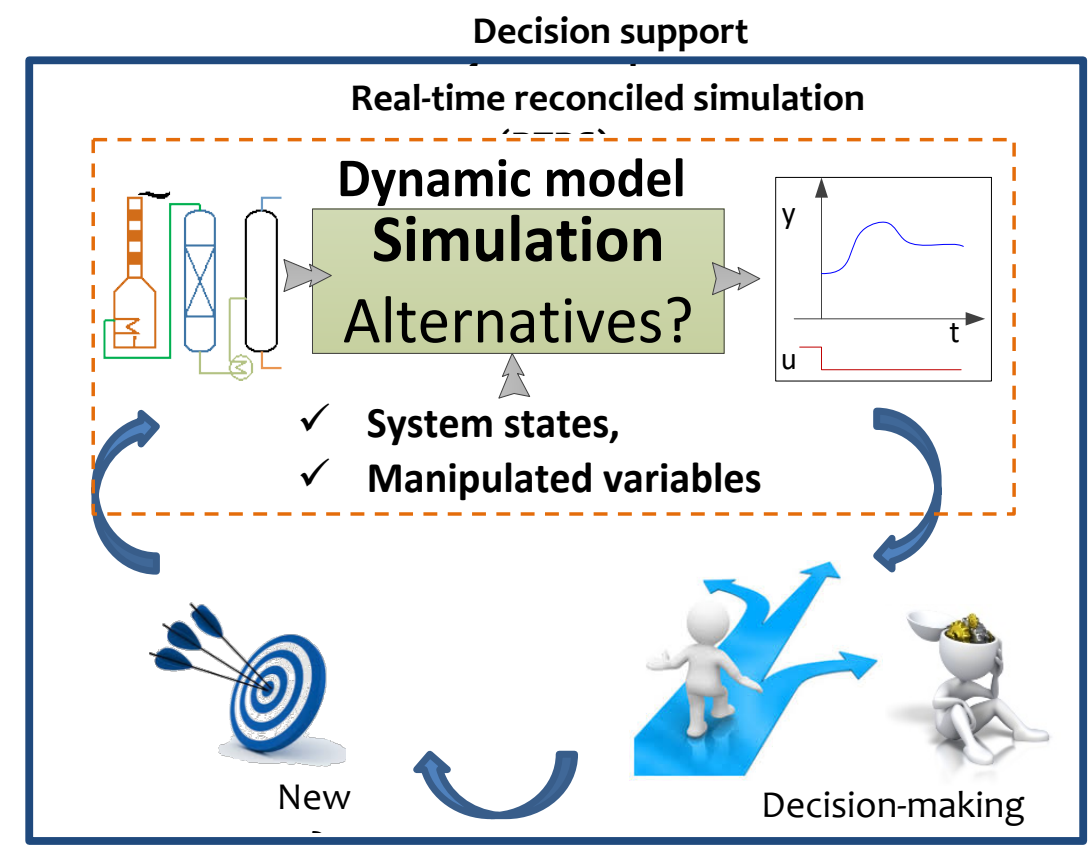

Fig. 3.1 - Decision support framework information flow with a real-time reconciled simulation application.

RTRS should well be visualized in an enterprise-wide decision support framework that includes, supply chain and production scheduling, machinery condition monitoring and maintenance, to mention two. Therefore, RTRS would take advantage of the most accurate data of machinery performance, or scheduled maintenance when estimating parameters and forecasting future process conditions. Similarly, current time estimations from RTRS may be available in real-time for scheduling and updating data for supply chain operations (Fig. 3.2). All relevant data from modules within the framework is exchanged through a data management system capable of storing historical data, as well as accessible form several platforms for data search. It is strongly believed, that the integration of tools such as RTRS with other decision-making modules across a business should enhance decision support potential at all levels enterprise-wide. Hence, there is growing interest from process industries in these applications in their transit heading to fully digitalized businesses, where digital twins play a central role. 
Enterprise-wide decision support framework

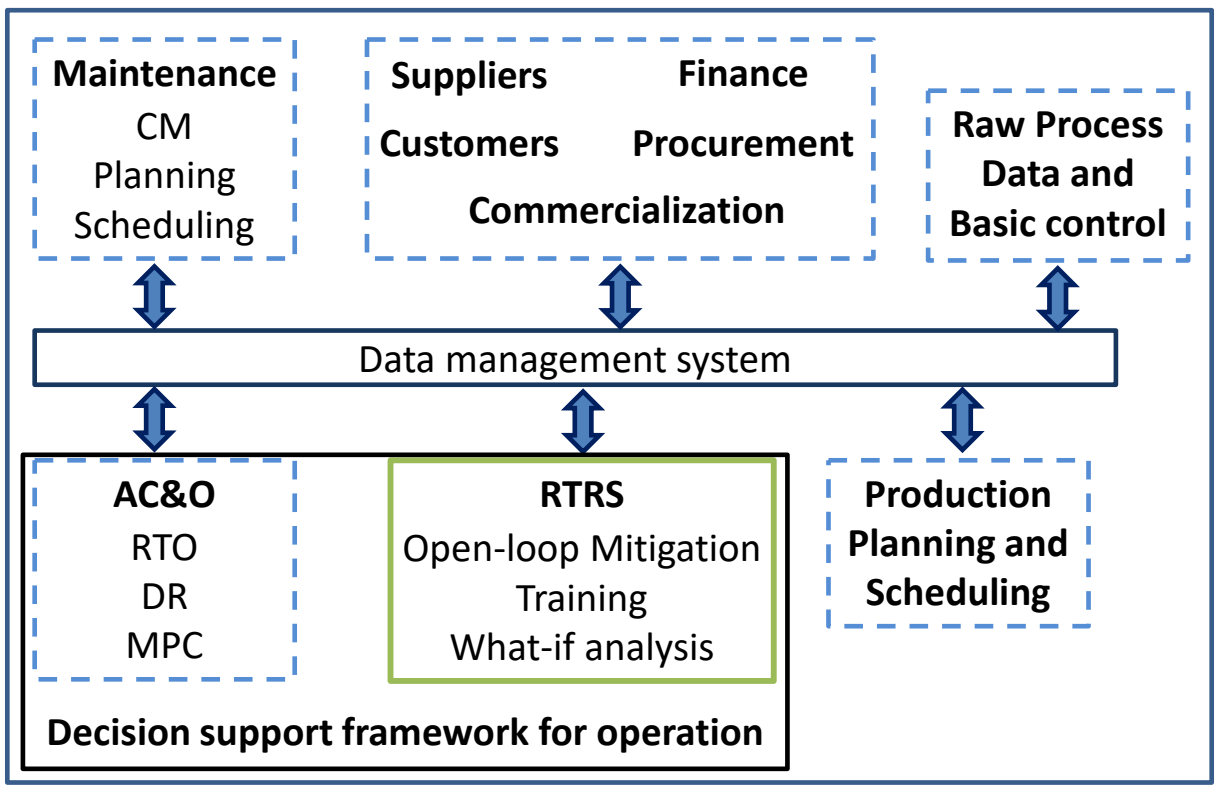

Fig. 3.2 - Enterprise-wide decision support framework, with RTRS highlighted in green. AC\&O: advanced control and optimization. CM: condition monitoring. DR: data reconciliation. RTO: realtime optimization. MPC: model predictive control.

\subsubsection{OBJECTIVE}

This chapter focuses on a real-time reconciled simulation, first principles modelbased (FPMb), decision support application for process operation, applied on an oil refinery hydrogen network case study. In particular, this work is an extension of previous research outcomes presented by Galan et al. (2019). All models used are developed using the dynamic simulation library detailed in the previous chapter.

\subsection{Problem STATEMENT}

\subsubsection{CONCEPTUAL DESCRIPTION}

The problem framework addressed in this work is given by a dynamic process system (DS) with online measurements (In) and their corresponding historical data. DS has its FPM representation as a differential-algebraic equations system (DAEs), dynamic model 
(DM) for short, which includes the following important features, as shown in Fig. 3.3: manipulated variables $(u)$, model outputs $(y)$, parameters $(p)$, disturbances $(w)$ and firstprinciple equations (FPE). In addition, Fig. 3.3 presents a schematic illustrating of all the previous components of the system in question.

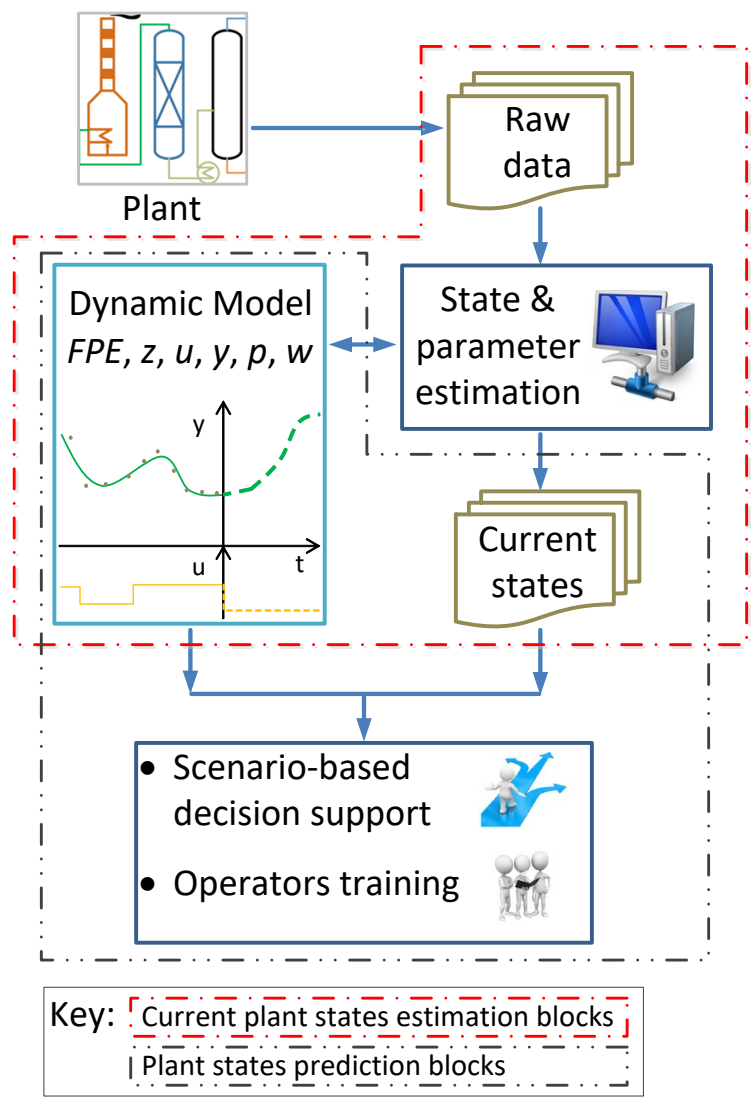

Fig. 3.3 - Framework and information flowchart addressed. FPE: first-principle equations. z: states. u: manipulated variables. y: model outputs. p: parameters. w: disturbances.

In this context, a real-time reconciled simulation tool should be able to estimate current time states and parameters, based on online and historical measurements. Estimations should support plant forecasting, aiding operators in their decision-making process for future actions, especially helping them assess proposed actions from other tools (e.g. RTO) or developing mitigation actions against unexpected changes when required. These might be intended for actual open-loop control action or to support scenario-based decisions, operators training or other forms of operation assessment. 
For the sake of clarity, the problem formulation is broken down into two subproblems: a state and parameter estimation, followed by a simulation problem, which might be simpler to digest than a monolithic approach (see Fig. 3.3). Conveniently, these sub-problems lead to different mathematical formulations using the same DM.

The state estimation is addressed applying the so called, and well known, moving horizon estimation (MHE) approach (Alessandri et al., 2010; Rawlings, 2009; Zavala et al., 2008). Thereafter, at current time values of states and parameters, the simulation is computed for forecasting future behavior of the system. License-free and efficient DAEs integration methods such as IDAS (Cao et al., 2003, 2002; Serban et al., 2019), are applied at this last step of the RTRS for solving the IVP.

\subsubsection{MATHEMATICAL FORMULATION}

\subsubsection{CURRENT STATE AND PARAMETER ESTIMATION}

A dynamic state estimation problem is presented in the following statement.

Given:

- a DAEs, i.e.: dynamic model (DM),

- $\quad$ plant measurements and information ( In, i.e.: raw plant data),

- $\quad$ previous manipulated variables' (MVs) values, $u$

Estimate current state variables such that DM variables response and plant data difference is minimized along a past rolling horizon of $\mathrm{N}$ sample times. Namely, solve the MHE.

Equations (3.1-6) show the mathematic formulation of the MHE problem, given a continuous-time dynamic system.

$$
y_{m}(t)=h(z(t), u(t), p)
$$




$$
\begin{aligned}
& f(z(t), \dot{z}(t), u(t), p, w(t))=0, z(0)=z_{0}, \\
& I_{k}^{(N)} \triangleq \operatorname{col}\left(y_{k-N}, \ldots, y_{k}, u_{k-N}, \ldots, u_{k-1}\right), \\
& \Delta w_{k}=w(k)-w(k-1) \\
& k=1,2, \ldots, N
\end{aligned}
$$

Where $z$ represents the state vector, $u$ the control vector and $w$ the unmeasured disturbance of the system, $\dot{z}$ is the derivative of $z$, and $p$ the vector of parameters (3.1). Model output $y$, with subscript $m$ refers to the model functional representation of an observed output variables as per (3.1). Additionally, the system data are collected through measurements vector $y_{k}$ (3.3). Notice that, at most $y_{k}$ vector (measurements) has the same components as $y_{m}$ for each time instant $t$. Typically, $y_{m}$ comprises a larger amount of components, simply due to impossibility or inconvenience of measuring all the properties represented in the model by $y_{m}$. The purpose is to estimate current states given past data over a "sliding time window" $[t-N, t]$, then $I^{(N)}{ }_{t}$ represents the information vector collected (3.3). Variables $w$, represented in the model system of equations (3.2), which are considered as disturbances due to various reasons, such as lack of measurements. Subscript ' $k$ ' refers to the time instant of the estimation of that variable within the time window in consideration.

Therefore, the minimization problem of the state estimation could be expressed as per (3.6-9).

$$
\min _{\left(\hat{z}_{t-N}, w_{N}, \cdots w_{k}, \hat{p}\right)}\left\{\left\|\hat{z}_{t-N \mid t}-\bar{z}_{t-N}\right\|_{P}^{2}+\sum_{i=k-N}^{k-1}\left\|\Delta w_{k-i}\right\|_{Q}^{2}+\sum_{i=k-N}^{k}\left\|y_{i}-y_{m}(i)\right\|_{R}^{2}\right\}
$$

Such that:

$$
z_{l b} \leq \hat{z}_{k} \leq z_{u b}
$$




$$
\begin{aligned}
& y_{l b} \leq y_{m} \leq y_{u b} \\
& \Delta w_{l b} \leq \Delta w_{k} \leq \Delta w_{u b}
\end{aligned}
$$

Where: $R, Q$ and $P$ are positive definite matrices that weight each term of the cost function. Subscripts $l b$ and $u b$, stand for lower bound and upper bound, respectively. Estimates other than disturbances $(w)$ are represented by a hat accent, such as $\hat{p}$ and $\hat{z}$, for parameters and states estimations respectively. The first term, represents the quadratic arrival cost (Alessandri et al., 2010; Arora and Biegler, 2001; Nicholson et al., 2014; Zavala et al., 2008), being $\bar{z}$ the previous estimation of $\hat{z}$. The second term accounts differences of unmeasured disturbances $\Delta w$ (3.4) along a time window $[t-N, N]$, this term prevents sudden changes in $\Delta w$. The third term represents the distance between actual measurements and estimations $(3.1,3.3)$.

Once the minimization problem is solved (3.6), $\hat{z}_{k-N}, w$ and $\hat{p}$ are used to integrate the model $f(3.2)$ from instant $k-N$ to $k$, and therefore estimate current $k$ instant states $\hat{z}_{k}$.

Fig. 3.4 presents a chart with $z, y, w, u$ and $t$, where past data (until $t-\mathrm{N}$ ) is used for the state and parameter estimation of current values (at $t$ ). These are represented by straight lines (continuous functions), and dots (discrete data or estimations). Dashed lines represent the evolution of the system in the future according to the simulation. Thereby, it is better visualized how the different stages in the RTRS are represented on the same timeline. 


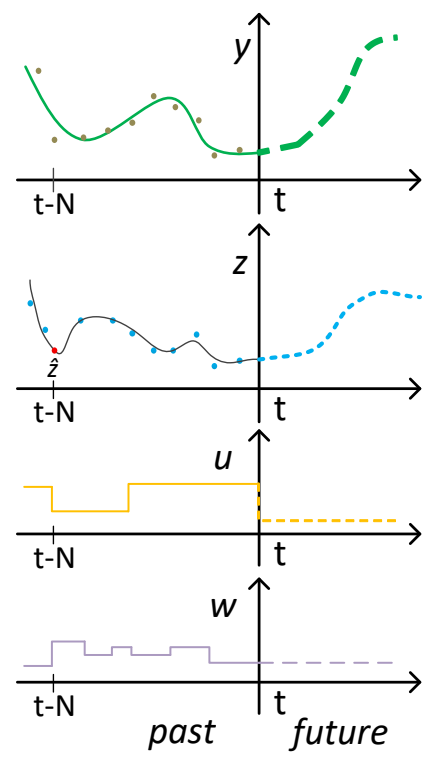

Fig. 3.4 - A representation of $y, z, u$ and $w$, on the same timeline. Full lines represent continuous variables in the past, while dashed lines represent those variables in the future. Dots correspond to discrete data or estimations in the past $t-N$ instants.

\subsubsection{FUTURE STATES PREDICTION AND SIMULATION}

Basically, the problem at this stage is formulated as a simulation considering previously determined states and parameters (see Fig. 3.4). A simulation is run to assess future states of the network under different meaningful scenarios, solving the DAEs, in this case from $t$ to $t_{\text {final, }}$ initiating at $\hat{z}_{t}$, and using previously determined $\hat{\mathrm{p}}$ and $w_{t}$. These scenarios should be deemed relevant for the case study, assessment, what-if analysis or any other final end of the RTRS.

\subsection{INTEGRATED MHE AND SIMULATION}

In order for satisfying both aspects of the problem, i.e. estimation and simulation features, a MHE embedded in a simulation environment is proposed, see Fig. 3.5. Thereby, a procedure for automatic estimation of current plant states and parameters, followed by dynamic simulation is implemented within the same software environment. The 
conceptual design and architecture presented along with its implementation in refinery hydrogen networks was previously introduced by Galan et al. (2019).

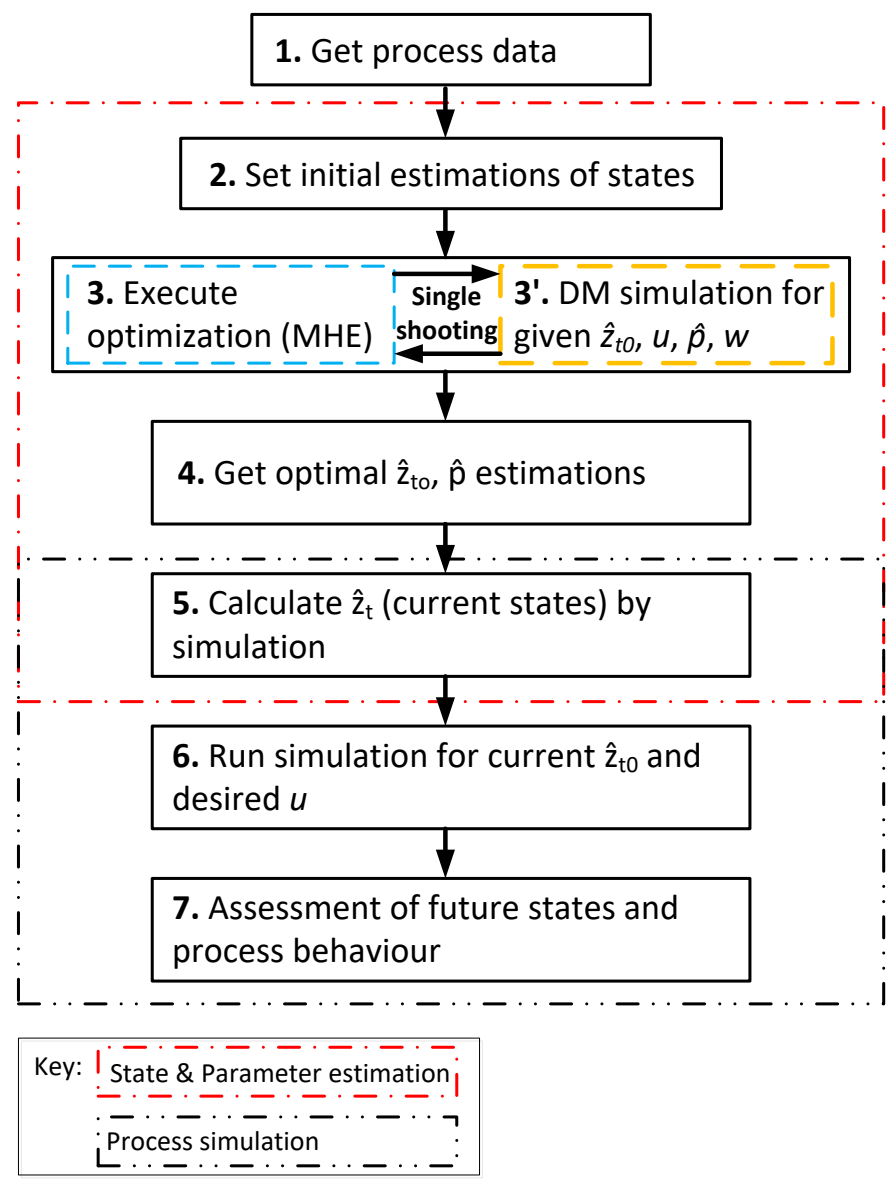

Fig. 3.5 - Generic procedure for the proposed MHE integrated in a simulation framework.

\subsubsection{PROCEDURE DESCRIPTION}

The calculation starts once actual measurements are available (step 1) and finalizes when the last simulation is run to predict future states (step 7). In Fig. 3.5 the main steps of the procedure are shown.

Steps 1-2 are required to run an NLP optimization, where $\hat{p}, \hat{z}_{t-N}$ and $w$ are the decision variables. Firstly, raw plant data is collected, which is typically available from a process historian server. Then, initial values for decision variables should be available for 
the DAEs and NLP solver. It should be noticed that, feasible initial values are required for this method to initialize properly. However, once started the algorithm calculates the values of states and parameters which are coherent and consistent with the model. Usually, estimations from previous executions or reconciled data records would provide good initialization points.

Steps 3-3' refer to the optimization itself to determine the states estimations, applying a single shooting sequential approach as described by Biegler (2010, chap. 9) . In this case, the gradients of the objective function and constraints w.r.t. $\hat{z}_{t-N}, \hat{p}$ and $\Delta w$ (decision variables, for short) are determined by the DAEs solver in the DM simulation, and passed on to the nonlinear programming (NLP) solver, as well as the initial values. The NLP solver returns back to the simulation with the decision variables, where the rest of the variables are determined (i.e.: $y_{m}, z$ ), as well as the objective function and constraints in (3.6-9). These runs iteratively governed by the optimization algorithm until reaching optimality tolerance or any other termination point of the NLP solver. A sketch of the sequence described is shown in Fig. 3.6. This optimization approach has the advantage of embedding naturally in a simulation framework, and therefore, enabling a straightforward execution of the whole process directly from the simulation software. In addition, the optimization problem itself is shrunk to the space of the decision variables alone, being the rest of the variables calculated at the DAEs instance for any given decision variables estimated. However, this may as well be a downside of the method, since it does not explode the full capacity of state-of-the-art NLP solvers, for which it would be required first and second order derivatives available to the optimization algorithm. One broadly use approach to overcome this issue is to apply full space discretization with automatic differentiation, however this kind of method have the disadvantage of weak, if any, embedded capability with simulation environments. A thorough and insightful discussion of advantages and shortcomings of these dynamic optimization approaches, and how they apply to state estimation and optimal control is presented by Biegler (2010, chaps. 8-9). This is beyond the scope of this thesis. 
Steps 4-5 take care of the last stage of the current state and parameter estimation. First, with the decision variables a simulation from $t-N$ to $t$ is executed to obtain $\hat{z}_{t}$ (estimation of current state). Notice that $\hat{p}$, by definition, is time independent, therefore valid and constant for the whole simulation time window, i.e. $[t-N, t]$.

Thereafter, in steps 6-7, $\hat{z}_{t}$ is used as initialization for process forecasting, where the DAE solver is given a set of future $u$ depending on the purpose of the simulation and the set of scenarios considered. These scenarios should be deemed relevant for the case study, assessment, what-if analysis or any other final end of the RTRS.

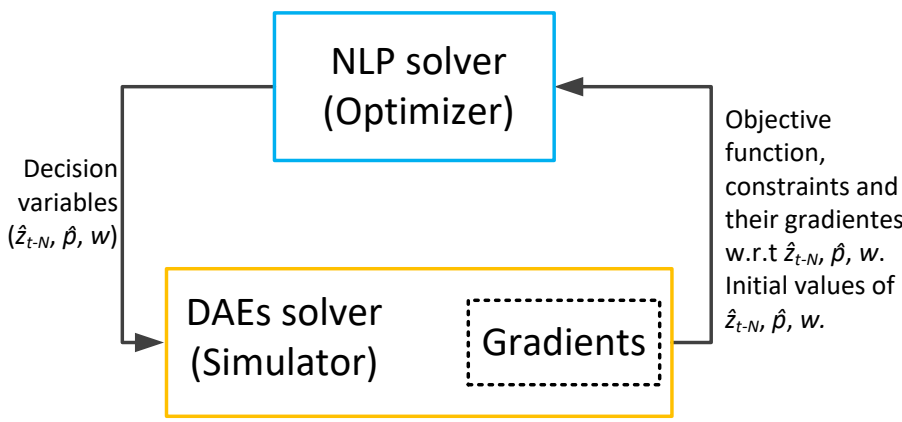

Fig. 3.6 - Representation of single shooting sequential approach used for solving the MHE, i.e.: dynamic optimization problem, for $\hat{z}_{t-N}, \hat{p}, w$ (decision variables, for short).

\subsubsection{SOFTWARE IMPLEMENTATION}

The RTRS is implemented on EcosimPro ${ }^{\circledR}$ / PROOSIS $^{\circledR}$ (EA Internacional, 2019a), based on first principle models built using the dynamic simulation library (described in chapter 2), which represent refinery hydrogen network components, and utilizes SNOPT (Gill et al., 2005, 2018) as NLP solver. EcosimPro ${ }^{\circledR} /$ PROOSIS $^{\circledR}$ is an equation and object oriented (EOO) simulation software, with straightforward communication capacity with other software packages, such as MS Excel ${ }^{\circledR}, \mathrm{C}++$. In particular, for the RTRS applications it is exploited its capability of building stand-alone simulations that can be run from MS Excel $^{\circledR}$ (named decks), where all initial values of variables is assigned. Therefore, spreadsheets play the interface role between plant raw data and actual RTRS application, bridging both sources. 
Consequently, the RTRS application is based on the procedure presented in Fig. 3.5, and considering the specifics of EcosimPro ${ }^{\circledR} /$ PROOSIS $^{\circledR}$ (EA Internacional, 2019b; Vázquez et al., 2010). In Fig. 8 is presented the architecture of the application developed in EcosimPro ${ }^{\circledR} /$ PROOSIS $^{\circledR}$. Although a detailed explanation of the software is out of the scope of this work, the main features are described for the sake of clarity and judgement independence of the reader. In addition, Vázquez et al. (2010) is recommended as a tutorial introduction to EcosimPro ${ }^{\circledR} / \mathrm{PROOSIS}^{\circledR}$.

The actual FPM is coded in a component of acasual equations (Fig. 3.7, full line blue box), which is further converted in a mathematical model once the user produces a partition by selecting the desired component boundaries set (Fig. 3.7, dashed line blue box). This partition can be assigned to a class instance (Fig. 3.7, full line orange box) or used for execution of experiments, where actual simulation routines take place. Is at this level where DAEs solver and external dynamic link libraries (dII) such as NLP solver can be called (EA Internacional, 2019b; Vázquez et al., 2010). In summary, an executable model has these three levels of coding within EcosimPro ${ }^{\circledR} /$ PROOSIS $^{\circledR}$. Namely, the model itself is coded in a component, and classes enable the integration of external dlls such as SNOPT engine (Gill et al., 2005, 2018). A sketch of the modules and how they relate to the RTRS procedure, described in Fig. 3.5, is shown in Fig. 3.7 for the sake of clarity.

It is important to notice that, in the proposed scheme signal noise is neglected as plant data is sourced in average form (e.g. 5 min average) from a historian server. In addition, unmeasured disturbances are directly calculated in $w$. In regards to gross errors, these are not calculated directly in this RTRS formulation for simplicity (it may well be incorporated in the future). Instead, these are accounted indirectly by using off-set values of instrument readings, which are worked out from steady state reconciliation data sourced externally. In this case, this is proven practical because the RTRS is used alongside a previously implemented steady state real-time data reconciliation (DR) tool. This DR tool provides robust estimations of streams and model parameters in steady state at two-hour time intervals, which are used conveniently to estimate off-set values in instrument 
readings. A more detailed description of the DR is addressed in chapter 4 along with how gross errors are accounted in the robust estimators.

Finally, the simulation itself is actually run through an experiment (executable module) from where the user can set up the integration time, boundaries and control variables (CV). This work presents two RTRS examples for demonstrative and discussion purposes. These are based on to two case studies: crude oil medium distillate hydrodesulfurization (HDS) plant (case I), and oil refinery hydrogen network (case II). The main characteristics are shown in Fig. 3.7.

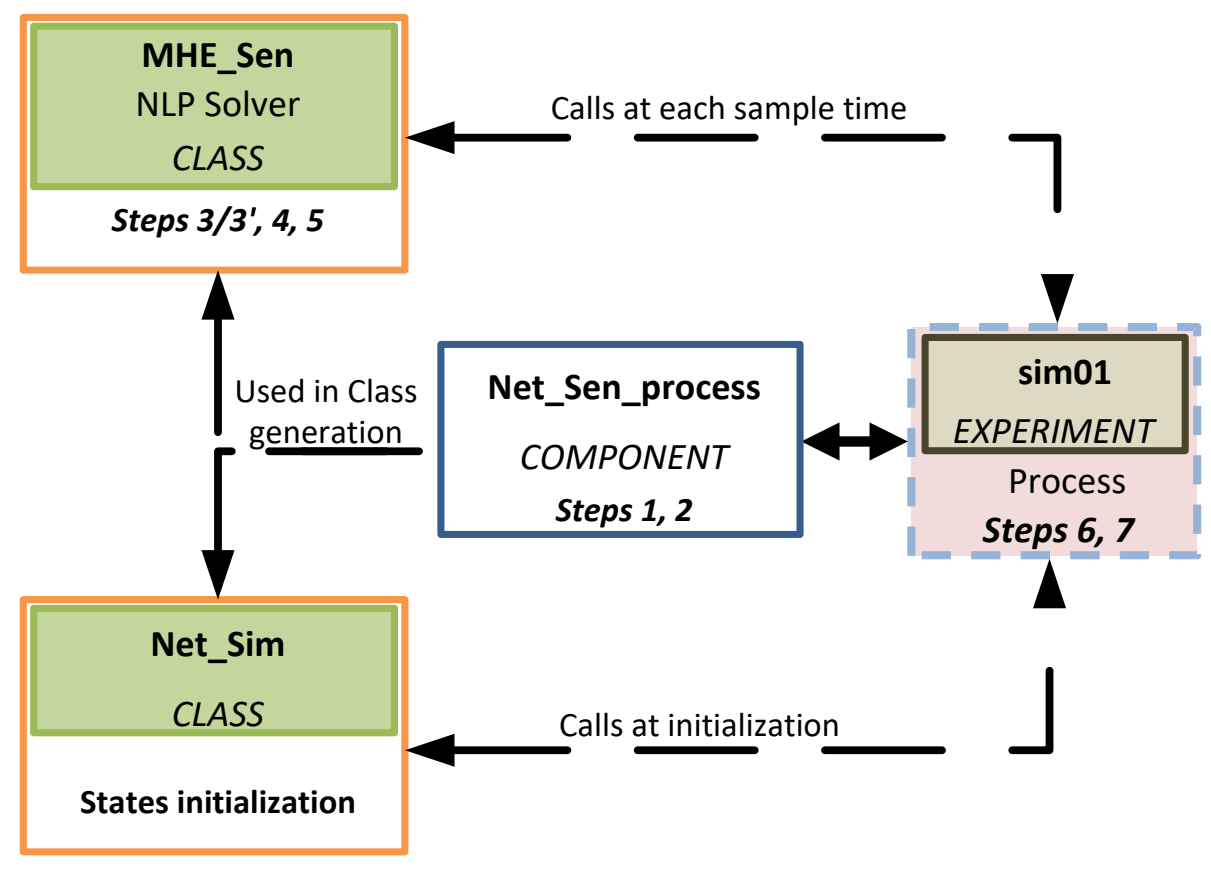

Fig. 3.7 - Scheme of real-time reconciled simulation (RTRS) implemented on EcosimPro ${ }^{\circledR}$ / PROOSIS $^{\circledR}$. Full blue box, represents the component where the FPM resides. Dashed blue box, represents a partition generated from the component once boundaries of the model are given, generation the full set of mathematical equations to be solved by the DAEs solver. Full brown box, represents an experiment from where the actual simulation is executed. Full orange box, represents a partition containing a class which is used for executing NLP routines at each integration interval of the DAEs. 


\subsubsection{WHAT-IF ANALYSIS}

A scenario-based analysis comes naturally with the idea of simulation, and in this case, RTRS enhances the impact of the analysis by the incorporation of current plant states and parameters. Furthermore, in this study a what-if analysis (WiA) is loosely defined as a scenario-based analysis in which the scenarios represent potential process or machinery degrading conditions. The analysis focuses on the best set of actions for each given scenario, and those simulations results, e.g.: forecasted states of the plant. In contrast, other scenario-based studies may rely on hazardous process conditions, or even purely normal operation events.

In this context, WiA is used for assessing the usefulness of RTRS applied to the case studies. In addition, one of the central features of RTRS is its simplicity, and straightforwardness, towards its integration with the plant information system, and graphic interface. Moreover, the control room schematics can easily be reproduced in the RTRS application, supporting a quick familiarization of operators with the tool.

\subsection{CASE STUDIES}

RTRS case studies are inspired in actual HDS plants and Petronor crude oil refinery hydrogen network, in Muskiz, Spain, though for confidentiality reasons either figures or configuration have been modified. In any case, both case studies represent fairly accurately process systems dynamics, which is deemed sufficient for demonstrating the usefulness of RTRS as a decision support tool.

\subsubsection{HDS PROCESS AND HYDROGEN NETWORK}

Hydrogen $\left(\mathrm{H}_{2}\right)$ is used in oil refineries mainly for removing sulfur from hydrocarbons in order to comply with environmental regulations (known as hydrodesulfurization process). In addition, hydrogen is consumed for converting "heavy" long chain hydrocarbons (least valuable), coming from vacuum distillation, into shorter chain and more volatile (a.k.a. "light") molecules (known as hydrocracking process), which 
are more valuable fuel blending components. Hence, $\mathrm{H}_{2}$ has become a key utility in the operation of the refineries. Currently, high purity hydrogen is produced either by an external supplier and delivered by pipeline to the refinery network or internally from steam reforming plants. Another source of hydrogen are platformer plants, producing low purity hydrogen as a by-product of the octanizing process, aimed at increasing the octane number of gasolines. Whichever the source is, hydrogen is finally distributed to individual consumer plants through a set of headers and pipelines, in a complex network arrangement. For instance, Petronor hydrogen network comprising 14 consumer plants, two steam reformers and two platformers, is presented in Fig. 3.8 (bottom). Moreover, headers are usually sorted by $\mathrm{H}_{2}$ purity, namely: high (HPH), low (LPH). HPH contain $\mathrm{H}_{2}$ purities over $90 \%$ and are fed by steam reformers. LPH contain over $70 \% \mathrm{H}_{2}$ purity and are fed by platformers and recycle purge from consumers, see Fig. 3.8 (top). Typically, excess gases from LPH and consumers, as well as other non-recyclable off-gases (e.g.: less than $70 \% \mathrm{H}_{2}$ purity), are collected by a fuel gas header (FGH) and used as fuel for burners across the refinery. Thus, only HPH and LPH gases are used as make-up (MU) gas to the consumer units.

A simplified schematic of a typical HDS plant can be seen in Fig. 3.9. The high sulfur hydrocarbon $(\mathrm{HC})$ feed is mixed with treatment gas (typically around $85-90 \%$ hydrogen content, and high pressure) coming from different sources (high and low purity headers, $\mathrm{HPH}$ and LPH respectively). This cold mixed stream is heated to reaction temperature, around $300-350^{\circ} \mathrm{C}$, by heat exchangers and a furnace (load heating subsystem, LHS) before going into the reactor. This untreated hot stream reacts on the catalyst fixed bed of the reactor, where the actual desulfurization and other side reactions take place. Due to exothermicity of the reactions, the outlet stream is used to preheat the cold stream load in the heat exchangers within the LHS. The next stage of the process is the separation of gas and liquid, for this purpose the high pressure separator (HPS) is fed with the cooled reactor outlet, and produces two outlets:

- High pressure sour gas (rich in hydrogen sulfide, $\mathrm{H}_{2} \mathrm{~S}$ ), 
- Medium or low pressure mixed gas and HC.

Another key point related to the operation of the reactors is purity management. As mentioned before, the gas recycled from the separation units (HPS) has lower purity than the treatment gas fed to the reactor, but its purity can be increased using permeation membranes or, after being sent to $\mathrm{LPH}$, reused in other plants either directly or mixed with fresh hydrogen to increase its purity.

Proper management of the network requires deciding in real-time, according to the hydrogen demands from the reactors and variable hydrogen flows generated by the platformer plants, how much fresh hydrogen should be produced by each producer plant, and how to distribute the hydrogen through the network and internally in the consumer plants so that the losses to FG, or in general costs, are minimized. In addition, the operation of the network has to consider as the most important economic target the maximization of the hydrocarbon loads processed in the hydrodesulfurization plants, which may be limited by the hydrogen available and the production aims stablished by the planning of the refinery for the period under consideration. Notice that reducing losses of hydrogen to FG may increase the hydrocarbon processing if hydrogen is the limiting factor, which provides additional value to the optimal management of the network. Certainly, decisions must satisfy all process constrains imposed by the equipment, operation, safety, targets or quality. 


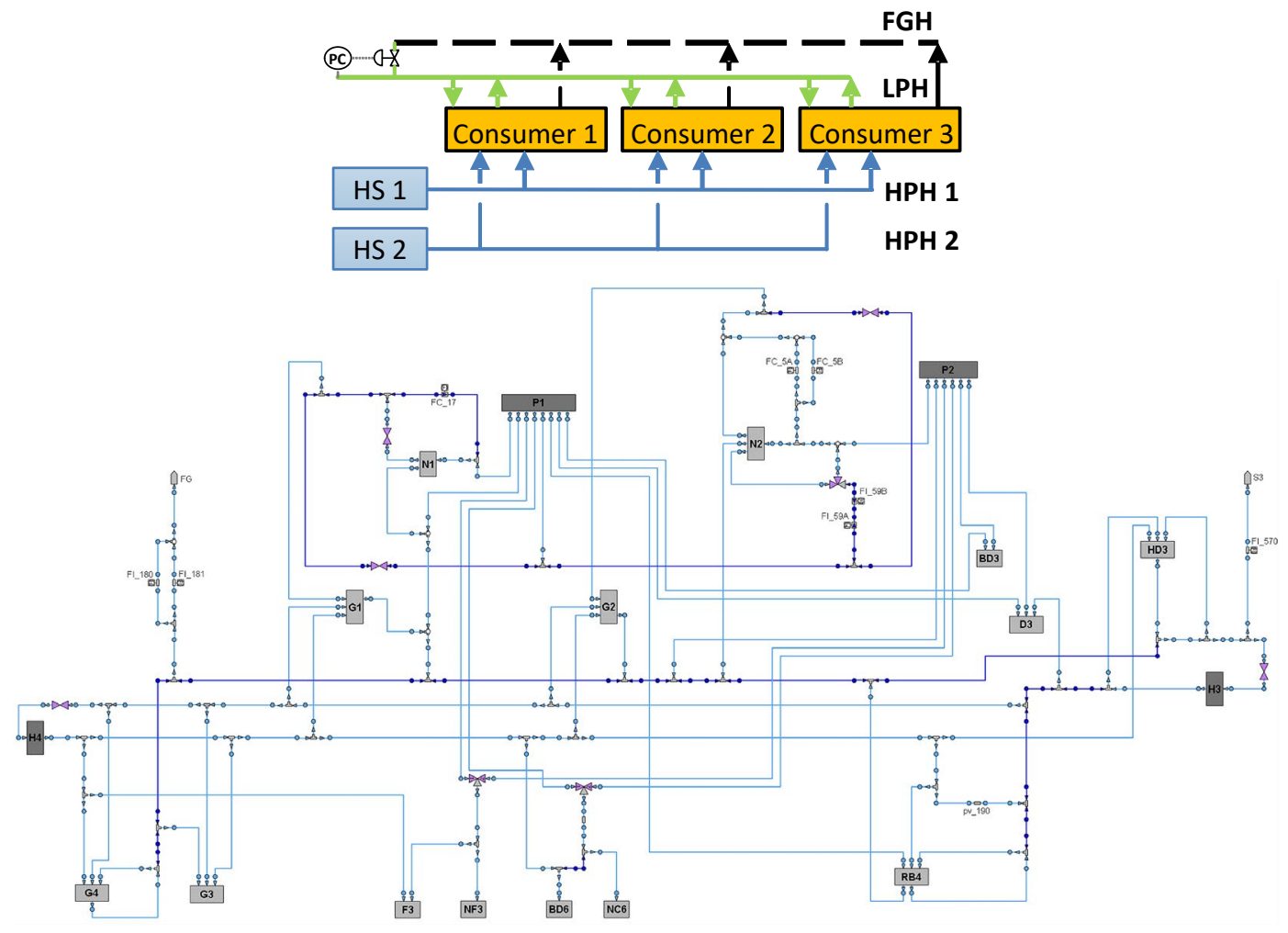

Fig. 3.8 - Top: generic $\mathrm{H}_{2}$ network diagram with sources (HS1/2), consumers 1-3, high purity headers (HPH 1/2), low purity header (LPH), and fuel gas header (FGH). Bottom: Petronor, refinery $\mathrm{H}_{2}$ network in Muskiz, Spain (Gómez Sayalero, 2016). 


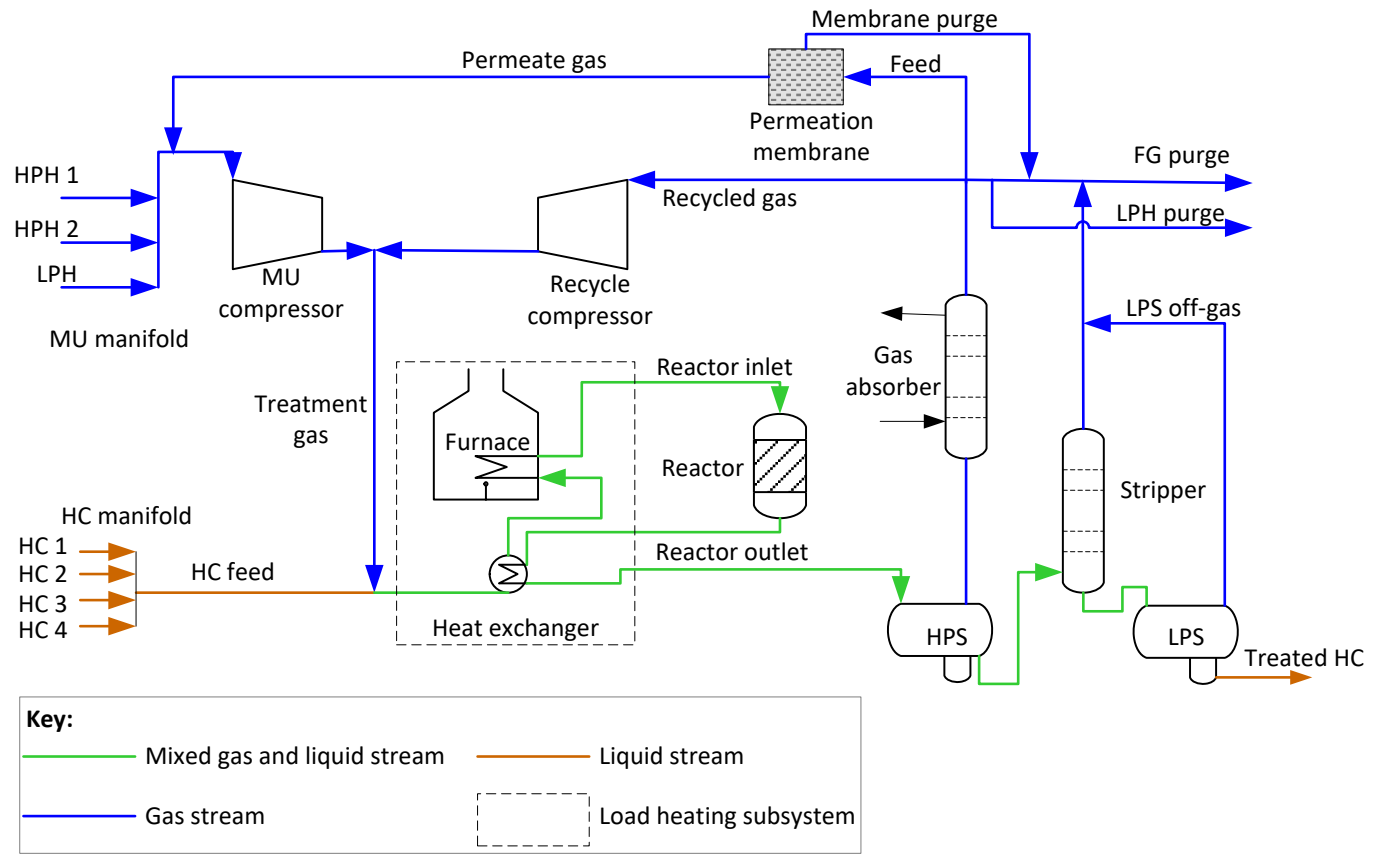

Fig. 3.9 - Simplified schematic of a generic hydrodesulfurization (HDS) plant. HPH: High purity gas header. LPH: Low purity gas header. HC: Liquid hydrocarbon; MU: Make-up. FG: Fuel gas. HPS: high pressure gas/HC separator. LPS: Low pressure gas/HC separator.

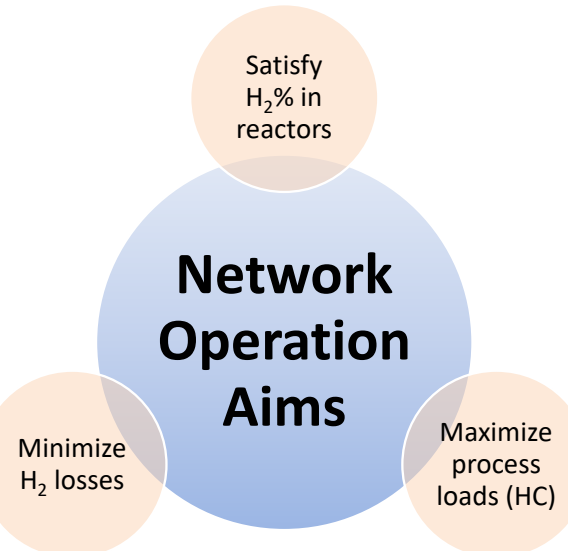

Fig. 3.10 - Hydrogen network operation aims: satisfying minimum $\mathrm{H}_{2}$ purity in reactors, maximizing $\mathrm{HC}$ processed (consistently with plant scheduling), and minimizing pure $\mathrm{H}_{2}$ losses to FGH.

In summary, hydrogen network operation aims should consider three dimensions, i.e.: $\mathrm{H}_{2}$ purity in reactors, $\mathrm{H}_{2}$ losses to $\mathrm{FGH}$ and $\mathrm{HC}$ processed in consumer plants. Due to the impact of each dimension in the process economy, an optimal operation follows the 
following broad directions (see Fig. 3.10): $\mathrm{H}_{2}$ purity in reactors and $\mathrm{H}_{2}$ losses, both to their lower bounds, and HC processed to its upper bound.

Actual networks probably comprise thousands of decision variables to be optimized at a time for a solution to be implementable. This does not necessarily translate into very complex optimization directions, especially if one is capable of identifying the mainstream optimization drivers. This simplifies significantly the comprehension of the full optimization problem solution and helps its communications across incumbent teams. Hereinafter, I summarize key optimization directions along with fundamental explanations of their impact in hydrogen network management, which help understand coincidences across design studies and the few actual operating case studies documented.

- Good management of recycle gas streams, especially of process units with the highest purity requirement. If units purge recycle gas, it should be collected in a lower purity header and distributed as make-up gas to process units with lower purity specifications. Ideally, units with high purity recycles should operate with closed purge valves to minimize their operating cost by saving fresh hydrogen in the front end. The reason for this is that fresh hydrogen is the main variable operating cost in hydrodesulfurization processes. Other costs are fuel gas for fired heaters' burners, electricity in compressors and steam.

- Extensive use of purification units, since these transform low purity hydrogen rich streams into high purity streams and fuel gas at negligible cost. Although purification units may not need to operate at full capacity in all scenarios, it is generally beneficial to operate them at high rates to reutilize low purity hydrogen to supply high purity consumers at minimum operating cost. Some exceptions to this arise, for example, when units are operating at tight purity upper bounds, as in the case of processes with centrifugal recycle compressors and relatively low purity requirements. Another, example of exception would be when fresh hydrogen production is close to minimum operating rates that essentially do not allow fresh hydrogen production cuts. 
- Whenever possible, sort hydrogen consumers by purity requirement level and supply low purity consumers with recycled or low purity hydrogen. This reutilizes hydrogen rich stream at no extra cost.

- It is usually beneficial to segregate purge and off-gas streams by purity, so that intermediate purity level headers are kept at high values even by purification means. This strategy may help save fresh hydrogen depending on network conditions.

Regardless of the approach utilized for addressing the hydrogen network problem, there is a consensus amongst researchers and practitioners with respect to the aforementioned mainstream optimization directions.

\subsubsection{MODEL DESCRIPTION}

A self-made refinery hydrogen dynamic simulation library (HDSLib) is used for case studies modeling. Therefore, all pieces of equipment and their connections are related to this library, which is built-for-purpose and supports most of the key process equipment in refinery hydrogen networks. A detailed description of this dynamic simulation library is addressed in chapter 2, including details of how is coded and degrees of freedom analysis of process equipment. Thereby, in this section only remarkable aspects of the model are highlighted for the sake of clarity. Chapter 2 should be revisited for equations references.

It is important to point out that, once the model is fully defined and ready for execution, it can easily be linked to an $\operatorname{Excel}^{\circledR}$ spreadsheet enabling data acquisition from a plant information system. This feature becomes of key importance when using the simulation with online data. This point is addressed in the following sections.

\subsubsection{MODEL HIGHLIGHTS}

It is important to bear in mind that, all pieces of equipment comprising the plants and their connections, as well as the network connections are formulated as a FPM using the previously mentioned HDSLib. This model is based on mass balances of hydrogen $\left(\mathrm{H}_{2}\right)$ and light ends (considered altogether as a single pseudo-component, $L I G$ ) at all nodes (N) 
of the network as in the pipelines, headers, and units as in (2.1, chapter 2$)$, where $F$ stands for gas flows, $X$ are hydrogen purities, and $M W$ refers to molecular weights.

In this model, any stream is fully defined by three degrees of freedom, these being different for gas and liquid fluids. Gas streams are defined by: $F$ (gas flowrate), $X_{H 2}\left(H_{2}\right.$ purity in gas stream) and $M W_{L I G}$ (molecular weight of light ends). Liquid streams are defined by: $H C$ (liquid hydrocarbon flowrate), $\rho_{H C}$ (HC density) and $M W_{H C}$ (HC molecular weight). Additionally, mixed gas and liquid streams are defined by: $F, X_{H 2}, M W_{L I G}, H C, \rho_{H C}$ and $M W_{H C}$. For details of utilized variables and equations revisit chapter 2, section 2.4 Library description.

The system integrator (DAE solver) has to work out all unknown variables, for a given set of boundaries and parameters of the system. Some of these are in turn estimated by the MHE routine within the RTRS procedure, while others are given by the user to represent known boundaries (e.g. $\rho_{H C}, M W_{H C}$ ). It is important to notice that, a stream has several variables associated and solving the model means that all of them are either calculated by the DAE solver (i.e. explicit, dynamic) or given by the user (e.g. boundaries). In addition, the RTRS application is providing a means of estimating states (dynamic variables) and parameters, such that plant data vs model mismatch is minimized. In other words, decision variables in the RTRS case studies are initial values at time $t-N$ of states (i.e. $\hat{z}_{t-N}$ ), model parameters (i.e. $\hat{p}$ ) and disturbance variables (i.e. $w$ ), see Fig 3.6 and Fig 3.7.

In addition to the set of equations presented in chapter 2 the model incorporates a quantification of process economy (3.10), which is used for assessing the impact of different sets of actions on profits over scenarios. In particular, for any given number of scenarios considered, the best alternative is the one that satisfies all operational constraints, at the time it provides the highest process profit. In this sense, is similar to a multi-stage stochastic programming problem, where the optimization takes into account only a discrete set of realizations of uncertainties. In RTRS, however, the best alternative 
comes from simulations of scenarios which should be conceptualized beforehand by the user.

$$
\operatorname{Profit}_{S C}=\sum_{i=1}^{U} p r_{i} \cdot H C_{S C, i}-\sum_{i=1}^{H} c t_{H S, i} \cdot H S_{S C, i}-\sum_{i=1}^{R H} c t_{L P H, i} \cdot L P H_{S C, i}
$$

Table 3.1 - Descriptions of variables and subscripts.

\begin{tabular}{|c|c|}
\hline$c t$ & Cost of gas stream in $\mathrm{k} € / \mathrm{Nm}^{3}$. \\
\hline$F$ & Gas flow, in $\mathrm{Nm}^{3} / \mathrm{h}$ \\
\hline G & Mixed gas and liquid stream, in $\mathrm{kmol} / \mathrm{h}$. \\
\hline$H C$ & Liquid hydrocarbons flow, in $\mathrm{m}^{3} / \mathrm{h}$. \\
\hline HS & High purity $\mathrm{H}_{2}$ source flowrate, in $\mathrm{Nm}^{3} / \mathrm{h}$. \\
\hline $\mathrm{ksol}_{\text {se, gasHC }}$ & Total gas solubility in hydrocarbons at separator se, in $\mathrm{Nm}^{3} / \mathrm{m}^{3} \mathrm{HC}$. \\
\hline $\mathrm{ksOl}_{\mathrm{se}, \mathrm{H} 2 \mathrm{HC}}$ & $\mathrm{H}_{2}$ solubility in hydrocarbons at separator se, in $\mathrm{Nm}^{3} / \mathrm{m}^{3} \mathrm{HC}$. \\
\hline $\mathrm{ksol}_{\text {se,MWLIG }}$ & $\mathrm{MW}_{\mathrm{LIG}}$ gas/liquid solubility coefficient, dimensionless. \\
\hline $\mathrm{ksOl}_{\mathrm{se}, \mathrm{H} 2 \mathrm{gas}}$ & $\mathrm{H}_{2}$ content in solubilized gas at separator se, in \%vol. \\
\hline$L M$ & Mol-Volume ratio for gases at $0^{\circ} \mathrm{C}$ and $1 \mathrm{~atm}, 22.414 \mathrm{Nm}^{3} / \mathrm{kmol}$. \\
\hline$L P H$ & Low purity gas pipeline header flowrate, in $\mathrm{Nm}^{3} / \mathrm{h}$. \\
\hline$M W$ & Molecular weight, in kg/kmol. \\
\hline$p r$ & Price of liquid hydrocarbon in $\mathrm{k} € / \mathrm{m}^{3}$. \\
\hline Profit & Profit of the process in $k € / h$. \\
\hline$r d$ & Specific demand $\left(\mathrm{rd}_{\mathrm{H} 2}\right)$ or generation $\left(\mathrm{rd}_{\mathrm{LI}}\right)$, in $\mathrm{Nm}^{3} \mathrm{H}_{2} / \mathrm{m}^{3} \mathrm{HC}$. \\
\hline$R D$ & Reactor demand $\left(\mathrm{RD}_{\mathrm{H} 2}\right)$ or generation $\left(\mathrm{RD}_{\mathrm{LIG}}\right)$, in $\mathrm{Nm}^{3} / \mathrm{h}$. \\
\hline W & Mass flow, in kg/h. \\
\hline$x$ & H2 or LIG fraction in a gas stream, in \%vol. \\
\hline$Y$ & Total molar fraction of a gas and liquid stream, in $\%$. \\
\hline$\rho$ & Density of a liquid stream, in $\mathrm{kg} / \mathrm{m}^{3}$. \\
\hline
\end{tabular}




\begin{tabular}{|l|l|}
\hline \multicolumn{1}{|l|}{} & \multicolumn{1}{c|}{ Hydraulic time constant, in $\mathrm{h}}$. \\
\hline in & The variable represents an inlet. \\
\hline$k$ & A node within the network. \\
\hline$H$ & Set of high purity $\mathrm{H}_{2}$ sources across the network. \\
\hline$N$ & Model node. \\
\hline$O$ & The purified gas of a permeation membrane. \\
\hline out & The variable represents an outlet. \\
\hline$p g$ & The gas purge of a permeation membrane. \\
\hline Ib & Lower bound \\
\hline$r$ & A reactor within the network. \\
\hline$R H$ & Set of make-up gas streams from LPH across the network. \\
\hline se & A separator within the network. \\
\hline$S C$ & Scenario. \\
\hline$U$ & Set of process units across the network. \\
\hline$Z$ & Upper bound \\
\hline
\end{tabular}

Table 3.2 - Engineering units used, unless explicitly stated otherwise.

\begin{tabular}{|l|l|l|l|l|l|}
\hline Liquid flow & Gas flow & Gas purity & Molecular weight & Time & Cost / Profit \\
\hline $\mathrm{m}^{3}$ & $\mathrm{Nm}^{3} / \mathrm{h}$ & $\% \mathrm{vol}$ & $\mathrm{kg} / \mathrm{kmol}$ & $\mathrm{h}$ & Euros $/ \mathrm{h}$ \\
\hline
\end{tabular}

\subsubsection{PLANT AND NETWORK MODELS}

Two independent models, a plant (case I) and a network (case II), are presented in the framework of refinery hydrogen network case studies. These provide the baselines for analyzing different sets of actions towards process improvements. For this purpose, the impact on Profit (as defined in 3.10) for each set is accounted. 
All process units are modeled using the dynamic simulation library for refinery hydrogen networks, i.e. HDSLib, representing their actual network setting. In spite of that, only plant G3 results are presented (case I) for confidentiality reasons and simplicity, Fig. 3.11 shows the schematic of G3 as it is represented for RTRS.

In contrast, case II is based on three typical HDS plants and their corresponding hydrogen network configuration across a refinery. Rather than an actual process network, case II is a simplified representative example aimed at demonstrating RTRS usefulness at network level. HDS plants and hydrogen network are presented in Fig. 3.12 and Fig. 3.13.

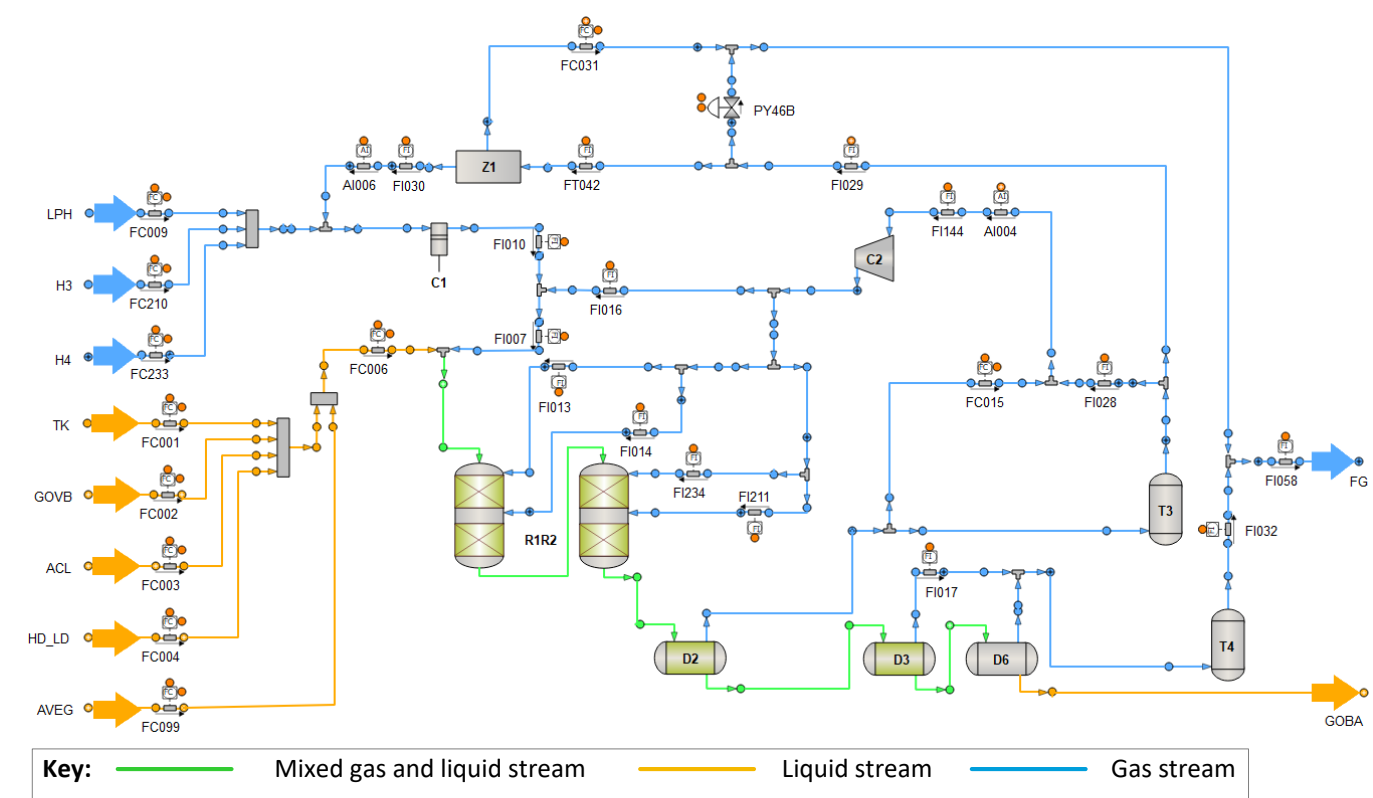

Fig. 3.11 - G3 medium distillate HDS flow diagram developed for RTRS. TK, GOVB, ACL, HD_LD, AVEG are HC feeds from different precedence. $\mathrm{H} 3$ and $\mathrm{H} 4$ are HPHs of steam reforming units. $\mathrm{C} 1$ : make-up reciprocating compressor. C2: recycle compressor. R1R2: hydrotreating reactors R1 and R2. D2: high pressure separator. D3: medium pressure separator. D6: low pressure separator. T3: amine absorption column. T4: low pressure off-gas column. Z1: H2 permeation membrane unit. 


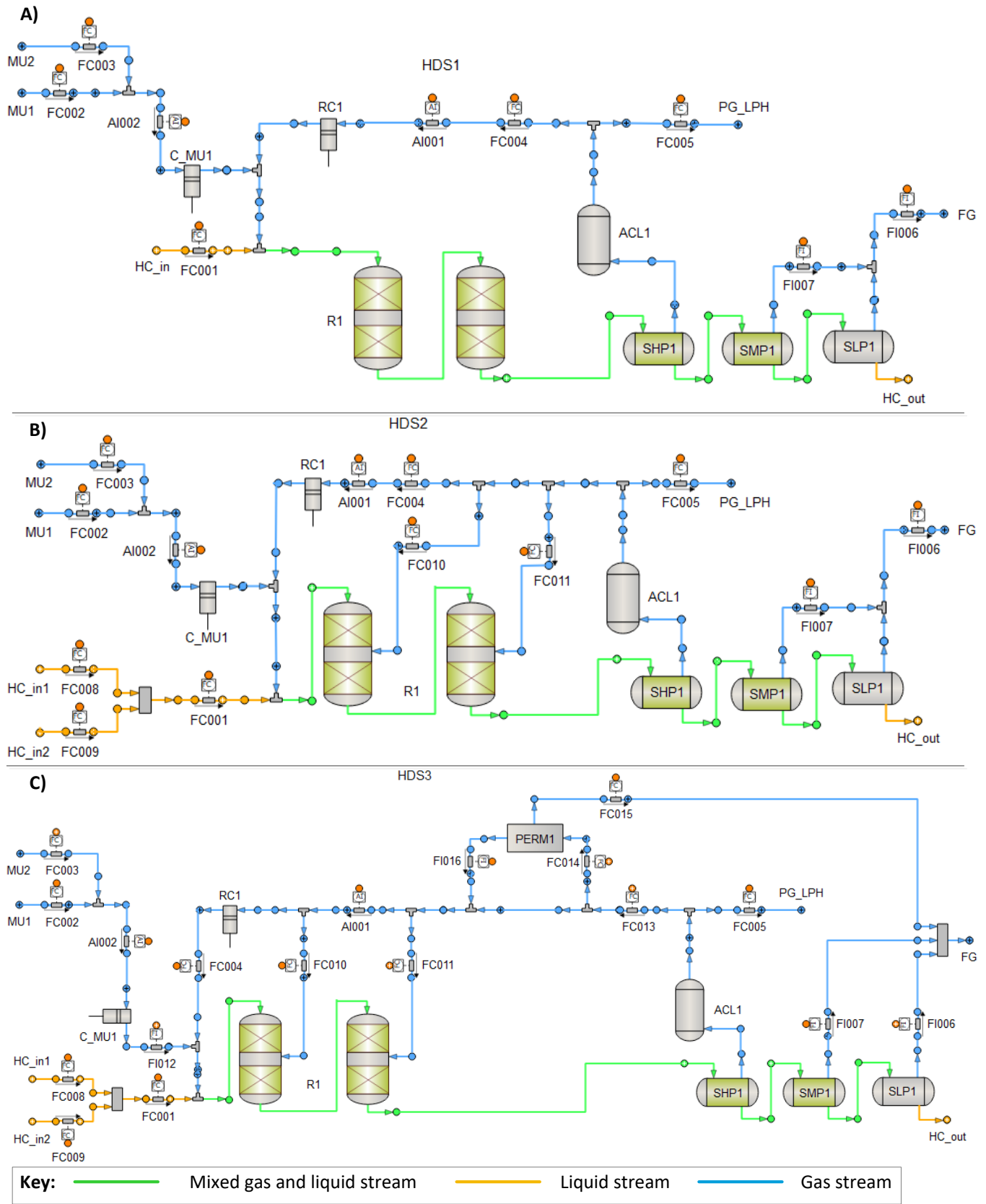

Fig. 3.12 - Flow diagrams of case study B HDS plants, i.e.: HDS1-3. A) HDS1. B) HDS2. C) HDS3. 


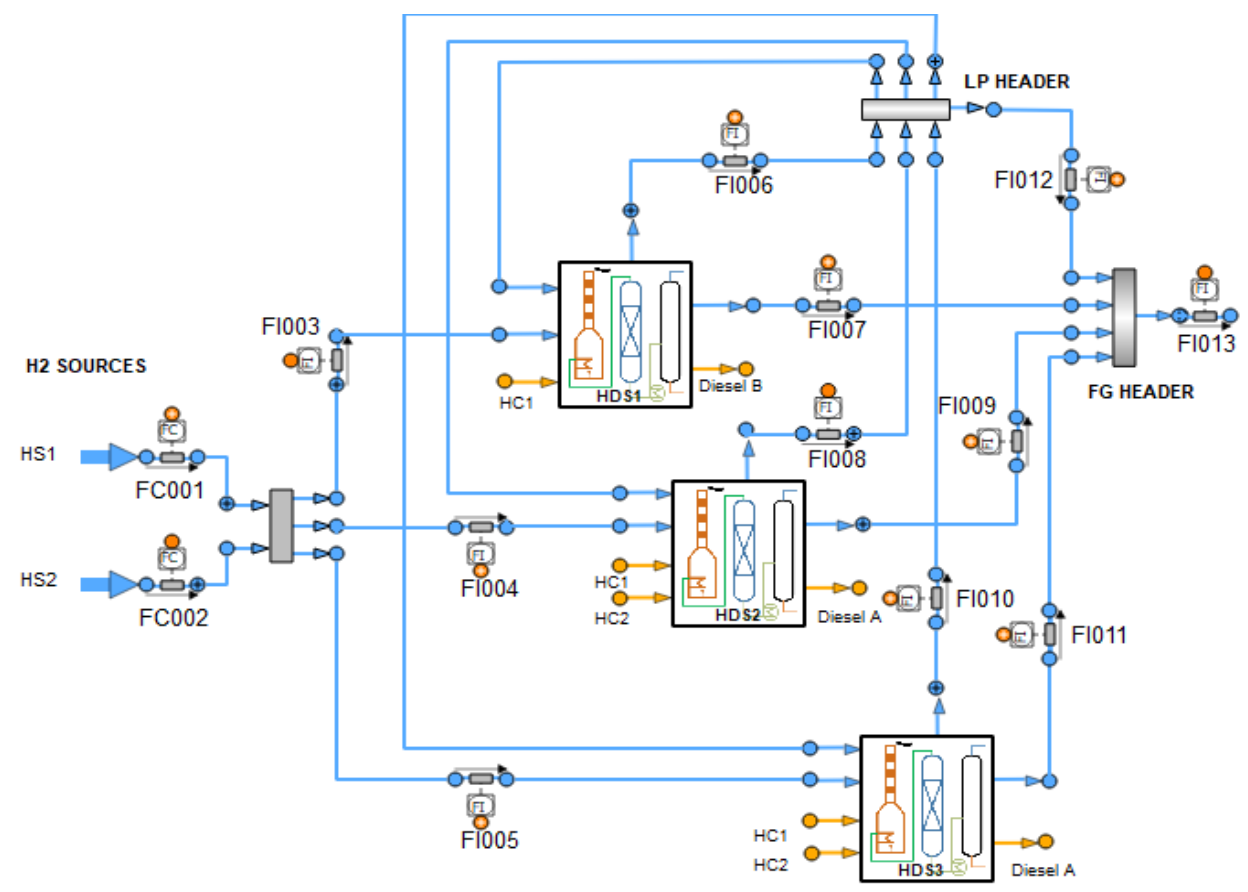

Fig. 3.13 - High level schematic of hydrogen network (case II). Two hydrogen sources: HS1 and HS2. One low purity header (LPH), aimed at collecting plant's recycle loop purges and feed them back to the process make-up. One fuel gas header (FGH), aimed at collecting gases from low and medium pressure separators at plant level, and LPH purge.

\subsubsection{SETS OF ACTIONS ASSESSED}

For each case study (I and II), a What-if analysis (WiA) based on three sets of actions is presented, these are: baseline (BL), actions $A(S A)$ and actions $B(S B)$. The baseline set, represents the process variables before and after a change of condition, without any additional corrective actions from the operators, where at least one operational constraint is not satisfied. Therefore, is the WiA input, giving meaning to the analysis by setting a constraint violation situation. While, SA and SB are, independent feasible operation strategies proposed by an operator applying RTRS, aimed at mitigating undesirable change of conditions. Table 3.3 summarizes the main features of each case study: baseline conditions, impact on constraints and analyzed sets of actions for mitigation. 
Table 3.3 - Summary of case studies conditions and mitigation sets of actions. *Stream or equipment related to the variable is between square brackets in italics. [R1R2] $\mathrm{rd}_{\mathrm{H} 2 \mathrm{SS}}$ changes from $65 \mathrm{Nm}^{3} \mathrm{H}_{2} / \mathrm{m}^{3} \mathrm{HC}$ to $75 \mathrm{Nm}^{3} \mathrm{H}_{2} / \mathrm{m}^{3} \mathrm{HC} @ 0.2 \mathrm{~h}$.

\begin{tabular}{|c|c|c|c|c|}
\hline \multirow[b]{3}{*}{$\begin{array}{l}\text { Baseline } \\
\text { (BL) }\end{array}$} & \multicolumn{2}{|l|}{ Case I (G3 plant) } & \multicolumn{2}{|l|}{ Case II (Network) } \\
\hline & Description & Variable* & Description & Variable* \\
\hline & $\begin{array}{l}15 \% \quad \mathrm{H}_{2} \\
\text { increase }\end{array}$ & {$[R 1 R 2] r d_{H 2 S S}$} & $\begin{array}{l}5 \mathrm{~m}^{3} / \mathrm{h} \mathrm{HC} \text { load increase } \\
\text { (in HDS1) }\end{array}$ & {$[F C 001] H C$} \\
\hline Violation & $\begin{array}{l}\text { Recycle gas purity LL } \\
\text { violation }\end{array}$ & {$[A / 004] X_{H 2}$} & $\begin{array}{l}\text { Recycle gas purity LL } \\
\text { violation }\end{array}$ & {$[A / 001] X_{H 2}$} \\
\hline $\begin{array}{l}\text { Mitigation } \\
\text { SA }\end{array}$ & $\begin{array}{l}\text { Membrane } \\
\text { increase }\end{array}$ & {$[F T 042] F$} & $\begin{array}{l}\text { 1. HDS1 HPH } \text { make-up } \\
\text { rise } \\
\begin{array}{llll}\text { 2. HDS1 } & \text { HC } & \text { load } \\
\text { decrease } & & \end{array}\end{array}$ & $\begin{array}{l}\text { 1. }[F I 003] F \\
\text { 2. }[F C O 01] H C\end{array}$ \\
\hline $\begin{array}{l}\text { Mitigation } \\
\text { SB }\end{array}$ & HPH make-up increase & {$[F C 233] F$} & $\begin{array}{l}\text { 1. HDS1 HPH make-up } \\
\text { rise } \\
\text { 2. HDS2 HPH make-up } \\
\text { decrease }\end{array}$ & $\begin{array}{l}\text { 1. }[F I O 03] F \\
\text { 2. }[F I O 04] F\end{array}$ \\
\hline
\end{tabular}

In addition, a qualitative analysis of the main streams and variables of case $\mathrm{I}$ is presented in Table 3.4. These include descriptions of variables and their main impact in the process and its economy. Variables fall into one of the following groups: manipulated variables (MV), controlled variables (CV), disturbance variables (DV) and measurements. At the same time, MV are boundaries of the system, while CV are explicit variables. Moreover, decision variables of the RTRS are identified as outputs (OP) in the variables' list.

Essentially, the analysis is focused on determining, the system state from plant data and simulate suitable mitigation sets of actions (SA and SB) by running a RTRS application. For didactic purposes a reduced set of variables (see Table 3.3) is presented in the analysis, however, in actual practice RTRS users are encouraged to consider all potential variables in the analysis. This approach is intended to support operators in their decision- 
making process, such that a negative change of condition is mitigated in the most costeffective way, considering Profit and process specification.

Table 3.4 - Main variables and streams of case I, their tags, description and impact. *Described in Table 3.1. B: boundary variable. E: explicit variable. D: dynamic variable. P: parameter. OP: output of RTRS. MV: manipulated variable. CV: controlled variable.

\begin{tabular}{|c|c|c|c|c|c|c|}
\hline Stream / & Variable & Tag & \multicolumn{3}{|c|}{ Description } & \multirow{2}{*}{$\begin{array}{l}\text { Impact } \\
\mathrm{H}_{2} \text { and } \mathrm{HC} \text { balance }\end{array}$} \\
\hline TK & $H C, \rho, M W$ & FC001 & B & MV & HC load & \\
\hline GOVB & $H C, \rho, M W$ & $\mathrm{FCOO2}$ & $\mathrm{B}$ & MV & HCload & $\mathrm{H}_{2}$ and $\mathrm{HC}$ balance \\
\hline $\mathrm{ACL}$ & $H C, \rho, M W$ & FC003 & $\mathrm{B}$ & MV & $\mathrm{HC}$ load & $\mathrm{H}_{2}$ and $\mathrm{HC}$ balance \\
\hline HD & $H C, \rho, M W$ & FC004 & B & MV & HC load & $\mathrm{H}_{2}$ and $\mathrm{HC}$ balance \\
\hline AVEG & $H C, \rho, M W$ & FC005 & B & MV & HC load & $\mathrm{H}_{2}$ and $\mathrm{HC}$ balance \\
\hline H3 & $F, X_{H 2}, M W_{L I G}$ & $\mathrm{FC} 210$ & $\mathrm{~B}$ & MV & MU gas & $\mathrm{H}_{2}$ balance. High impact on purity. \\
\hline $\mathrm{H} 4$ & $F, X_{H 2}, M W_{L I G}$ & FC233 & B & MV & MU gas & $\mathrm{H}_{2}$ balance. High impact on purity. \\
\hline R1R2.Q1 & $F$ & $\mathrm{FIO13}$ & B & MV & Quench & Neutral \\
\hline R1R2.Q2 & $F$ & $\mathrm{FIO14}$ & $\mathrm{B}$ & MV & Quench & Neutral \\
\hline R1R2.Q3 & $F$ & $\mathrm{FI} 234$ & $\mathrm{~B}$ & MV & Quench & Neutral \\
\hline R1R2.Q4 & $F$ & $\mathrm{FI} 211$ & B & MV & Quench & Neutral \\
\hline $\mathrm{FI} 144$ & $F$ & FI144 & B & MV & Recycle & Neutral \\
\hline FT042 & $F$ & FT042 & $\mathrm{B}$ & MV & Membrane feed & $\mathrm{H}_{2}$ balance. High impact on purity. \\
\hline PY46B & $F$ & PY46B & $\mathrm{B}$ & MV & High pressure purge & $\mathrm{H}_{2}$ balance \\
\hline FC006 & $H C$ & FC006 & $\mathrm{E}$ & CV & $\mathrm{HC}$ load & $\mathrm{H}_{2}$ and $\mathrm{HC}$ balance \\
\hline $\mathrm{LPH}$ & $F$ & FC009 & $\mathrm{E}$ & CV & MU gas & $\mathrm{H}_{2}$ balance. High impact on purity. \\
\hline D2.Gas & $F$ & & $\mathrm{E}$ & $\mathrm{CV}$ & Off-gas & $\begin{array}{l}\mathrm{H}_{2} \text { balance. Highly sensitive to ksol } \\
\text { changes. }\end{array}$ \\
\hline D3.Gas & $F$ & $\mathrm{FIO17}$ & $\mathrm{E}$ & $\mathrm{CV}$ & Off-gas & $\begin{array}{l}\mathrm{H}_{2} \text { balance. Highly sensitive to ksol } \\
\text { changes. }\end{array}$ \\
\hline GOBA & $H C$ & & $\mathrm{E}$ & CV & Product & $\mathrm{HC}$ balance \\
\hline Al004 & $X_{\mathrm{H} 2}$ & Al004 & $\mathrm{E}$ & CV & $\mathrm{H} 2$ analyzer & $\mathrm{H}_{2}$ balance. Purity control point. \\
\hline $\mathrm{FIO16}$ & $F$ & FI016 & $\mathrm{E}$ & CV & Recycle tie-in & Neutral \\
\hline $\mathrm{FIO30}$ & $F$ & $\mathrm{FIO30}$ & $\mathrm{E}$ & CV & Membrane effluent & $\mathrm{H}_{2}$ balance \\
\hline Al006 & $X_{\mathrm{H} 2}$ & Al006 & $\mathrm{E}$ & CV & $\mathrm{H} 2$ analyzer & $\mathrm{H}_{2}$ balance \\
\hline FC031 & $F$ & FC031 & $\mathrm{E}$ & CV & Membrane purge & $\mathrm{H}_{2}$ balance \\
\hline $\mathrm{FIO32}$ & $F$ & $\mathrm{FIO32}$ & $\mathrm{E}$ & CV & Off-gas & $\mathrm{H}_{2}$ balance \\
\hline $\mathrm{FI058}$ & $F$ & $\mathrm{Fl058}$ & $\mathrm{E}$ & CV & Plant vent & $\mathrm{H}_{2}$ balance \\
\hline $\mathrm{LPH}$ & $F$ & FC009 & $\mathrm{E}$ & CV & MU gas & $\mathrm{H}_{2}$ balance. High impact on purity. \\
\hline
\end{tabular}




\begin{tabular}{|c|c|c|c|c|c|c|}
\hline R1R2 & $H C_{\text {out }}$ & & D & OP & * & $\mathrm{HC}$ and $\mathrm{H}_{2}$ balance \\
\hline R1R2 & $r d_{H 2}$ & & D & OP & * & $\begin{array}{l}\mathrm{H}_{2} \text { demand. High impact on } \\
\text { recycle purity. }\end{array}$ \\
\hline R1R2 & $r d_{L G}$ & & $\mathrm{D}$ & $\mathrm{OP}$ & * & LIG generation \\
\hline D2 & $H C$ & & $\mathrm{D}$ & $\mathrm{OP}$ & * & $\mathrm{HC}$ and $\mathrm{H}_{2}$ balance \\
\hline D2 & $k \mathrm{sO}_{\mathrm{H} 2 \mathrm{HC}}$ & & D & $\mathrm{OP}$ & * & Recycled $\mathrm{H}_{2}$ and losses to FG. \\
\hline D2 & ksolGASHC & & D & $\mathrm{OP}$ & * & Recycled gas and losses to FG. \\
\hline D3 & $H C$ & & D & $\mathrm{OP}$ & * & $\mathrm{HC}$ and $\mathrm{H}_{2}$ balance \\
\hline D3 & $\mathrm{ksOl}_{\mathrm{H} \mathrm{HC}}$ & & D & $\mathrm{OP}$ & * & $\mathrm{H}_{2}$ losses to $\mathrm{FG}$ \\
\hline D3 & $k_{S S O} I_{G A S H C}$ & & D & $\mathrm{OP}$ & * & Gas losses to FG \\
\hline R1R2 & $r d_{H 2 S S}$ & & $\mathrm{P}$ & $\mathrm{OP}$ & $*$ & Steady state $\mathrm{H}_{2}$ demand \\
\hline R1R2 & $r d_{\text {LISSS }}$ & & $\mathrm{P}$ & $\mathrm{OP}$ & * & Steady state LIG generation \\
\hline R1R2 & tau & & $\mathrm{P}$ & $\mathrm{OP}$ & * & Time to steady state \\
\hline D2 & ksolHzHCSS & & $\mathrm{P}$ & $\mathrm{OP}$ & * & $\begin{array}{l}\text { Steady state recycled } \mathrm{H}_{2} \text { and } \\
\text { losses to FG }\end{array}$ \\
\hline D2 & ksol GASHCSS & & $\mathrm{P}$ & $\mathrm{OP}$ & * & $\begin{array}{l}\text { Steady state recycled gas and } \\
\text { losses to FG }\end{array}$ \\
\hline D2 & tau & & $\mathrm{P}$ & $\mathrm{OP}$ & $*$ & Time to steady state \\
\hline D3 & ksol HzHCSS & & $\mathrm{P}$ & $\mathrm{OP}$ & * & Steady state $\mathrm{H}_{2}$ losses to $\mathrm{FG}$ \\
\hline D3 & ksOlGASHCSS & & $\mathrm{P}$ & $\mathrm{OP}$ & $*$ & Steady state gas losses to FG \\
\hline D3 & $\tan$ & & $\mathrm{P}$ & $\mathrm{OP}$ & $*$ & Time to steady state \\
\hline $\mathrm{LPH}$ & $X_{\mathrm{H} 2}$ & FC009 & $\mathrm{P}$ & $\mathrm{OP}$ & * & $\mathrm{H}_{2}$ balance. Disturbance. \\
\hline R1R2 & $M W_{\text {LIG }}$ & & $\mathrm{P}$ & $\mathrm{OP}$ & $*$ & Product density. Disturbance. \\
\hline
\end{tabular}

Table 3.5 - Main streams of case II at network level, their tags, description and impact. *Described in Table 1. B: boundary variable. E: explicit variable. D: dynamic variable. P: parameter. OP: output of RTRS. MV: manipulated variable. CV: controlled variable.

\begin{tabular}{|c|c|c|c|c|c|c|}
\hline Stream / & Variable & Tag & & scrip & & Impact \\
\hline $\mathrm{HS} 1$ & $\mathrm{~F}, \mathrm{X}_{\mathrm{H} 2}, \mathrm{MW}_{\mathrm{LIG}}$ & FC001 & $B$ & $\mathrm{MV}$ & HPH Source & $\mathrm{H}_{2}$ balance and purity. \\
\hline HS2 & MWLG & FCO02 & B & MV & HPH Source & Gas balance. \\
\hline FI003 & $\mathrm{F}$ & $\mathrm{FlO03}$ & B & MV & $\mathrm{MU}$ gas to HDS & $\mathrm{H}_{2}$ balance and purity. \\
\hline FI006 & $\mathrm{F}$ & FI006 & B & MV & Purge to LPH & $\mathrm{H}_{2}$ balance and purity. \\
\hline F1008 & $\mathrm{F}$ & Fl008 & B & MV & Purge to LPH & $\mathrm{H}_{2}$ balance and purity. \\
\hline FI010 & $\mathrm{F}$ & Fl010 & B & MV & Purge to LPH & $\mathrm{H}_{2}$ balance and purity. \\
\hline FIO04 & $\mathrm{F}$ & $\mathrm{FlOO4}$ & $\mathrm{E}$ & $\mathrm{CV}$ & MU gas to HDS & $\mathrm{H}_{2}$ balance and purity. \\
\hline
\end{tabular}




\begin{tabular}{|l|l|l|l|l|l|l|}
\hline FI005 & F & FI005 & E & CV & MU gas to HDS & $\mathrm{H}_{2}$ balance and purity. \\
\hline FI007 & F & FI007 & E & CV & Purge to FGH & $\mathrm{H}_{2}$ balance and purity. \\
\hline FI009 & F & FI009 & E & CV & Purge to FGH & $\mathrm{H}_{2}$ balance and purity. \\
\hline FI011 & F & FI011 & E & CV & Purge to FGH & $\mathrm{H}_{2}$ balance and purity. \\
\hline FI012 & F & FI012 & E & CV & LPH to FGH & $\mathrm{H}_{2}$ balance and purity. \\
\hline FI013 & F & FI013 & E & CV & FGH off-gas & $\mathrm{H}_{2}$ balance and purity. \\
\hline HS2 & F, $\mathrm{X}_{2}$ & FC002 & E & CV/OP & HPH Source & $\mathrm{H}_{2}$ balance and purity. \\
\hline HDS1.AI001 & $\mathrm{X}_{\mathrm{H} 2}$ & $\mathrm{Al001}$ & $\mathrm{E}$ & $\mathrm{CV}$ & $*$ & $\begin{array}{l}\mathrm{H}_{2} \text { balance. Purity control } \\
\text { point. }\end{array}$ \\
\hline HDS2.AI001 & $\mathrm{X}_{\mathrm{H} 2}$ & $\mathrm{Al001}$ & $\mathrm{E}$ & $\mathrm{CV}$ & $*$ & $\begin{array}{l}\mathrm{H}_{2} \text { balance. Purity control } \\
\text { point. }\end{array}$ \\
\hline HDS3.AI001 & $\mathrm{X}_{\mathrm{H} 2}$ & $\mathrm{Al001}$ & $\mathrm{E}$ & $\mathrm{CV}$ & $*$ & $\begin{array}{l}\mathrm{H}_{2} \text { balance. Purity control } \\
\text { point. }\end{array}$ \\
\hline
\end{tabular}

Case II has a homologous set of streams to case I at unit level, with the addition of network streams to interconnect all plants. Thereby, Table 3.5 presents key variables at network level and their descriptions, rather than a full list, in the interest of conciseness. Moreover, decision variables of the RTRS are analogous to Case I, considering Case II has three plants integrated in a network instead of only one as in Case I and one additional output (OP) of the RTRS needed to solve the system.

A summary of statistics of both models is shown in Table 3.6. These are intended to provide a broad idea of the size of the models in question.

Table 3.6 - Summary of models' statistics.

\begin{tabular}{|l|l|l|}
\hline Description & Case I & Case II \\
\hline Equations & 645 & 1305 \\
\hline Boxes (coupled subsystems of equations) & 3 & 5 \\
\hline Linear boxes & 2 & 4 \\
\hline Nonlinear boxes & 1 & 1 \\
\hline Input DATA & 290 & 592 \\
\hline Output EXPLICIT & 634 & 1273 \\
\hline
\end{tabular}




\begin{tabular}{|l|l|l|}
\hline Output DYNAMICS & 9 & 27 \\
\hline Output ALGEBRAICS & 2 & 5 \\
\hline Size of Jacobian matrix (DYNAMIC+ALGEBRAIC). & $11 \times 11$ & $32 \times 32$ \\
\hline Integration method & IDAS & IDAS \\
\hline
\end{tabular}

\subsubsection{ASSESSMENT OF THE ACCURACY}

Even before studying the results of the RTRS at any given condition it is important to assess the accuracy of the estimation during change of conditions, either manual changes or unexpected. In addition, the values in $P, Q$ and $R$ of (3.6) are adjusted in order to properly set up the parameter and state estimation module. First and foremost, predictions are to be useful if the estimations are within a sensible uncertainty range. Although a detailed study of the accuracy of the estimations and MHE calibration is outside the scope of this manuscript, a brief conceptual description is provided along with relative errors (w.r.t. actual values) of key variables tested for G3 (Case I) shown in Fig 3.14 .

Starting from reconciled values of the process, the RTRS is executed offline altering a number of variables such as hydrogen demand, hydrogen purity, HC load, among others. The test consists of simulating several changes in operating condition, both expected and unexpected, for a $2 \mathrm{~h}$ period of time. In this way, true values are known beforehand and used for calculating the relative error of variables. Simultaneously, the state estimation block of RTRS is run continuously, outputting estimates of states and parameters. In this way, the MHE is qualitatively validated, prior to its online use for any particular process unit. The main purpose of this validation is to adjust the state estimation routine to calculate values within reasonable error ranges. These results are shown in Fig. 3.14, quadrants $A$ to $D$.

The state and parameter estimation block of the RTRS presents satisfactory results in terms of estimation accuracy of the method, for all process conditions tested. The most remarkable outcome is probably that, even though errors vary widely across variables 
most of the errors are below $10 \%$ which is considered satisfactory in this type of gaseous industrial networks. Naturally, the more accurate the better, however these figures should only be taken as a first safe reference for validation of the estimations. Adjusting values in $R$ (3.6) is possible to relax or tighten the error for each measurement in the MHE block (estimation error over actual value in \%). Moreover, it is pointed out that key instrumentation should receive greater weight in $R$ in order for achieving the best results. In overall, the estimation itself is considered validated for the purpose of this study. Actual applications should undergo a rigorous validation process which would be fit to purpose, rather than following a general validation criteria. A detailed study of these aspects of the proposed methodology and analysis of the tuning process is out of the scope of this work.

It is important to bear in mind that sensible estimations are essential for further states predictions, and plant forecasting. Estimations of future variables, relies on current time estimations. Therefore, a thorough tuning process is advised during commissioning of any RTRS tool.
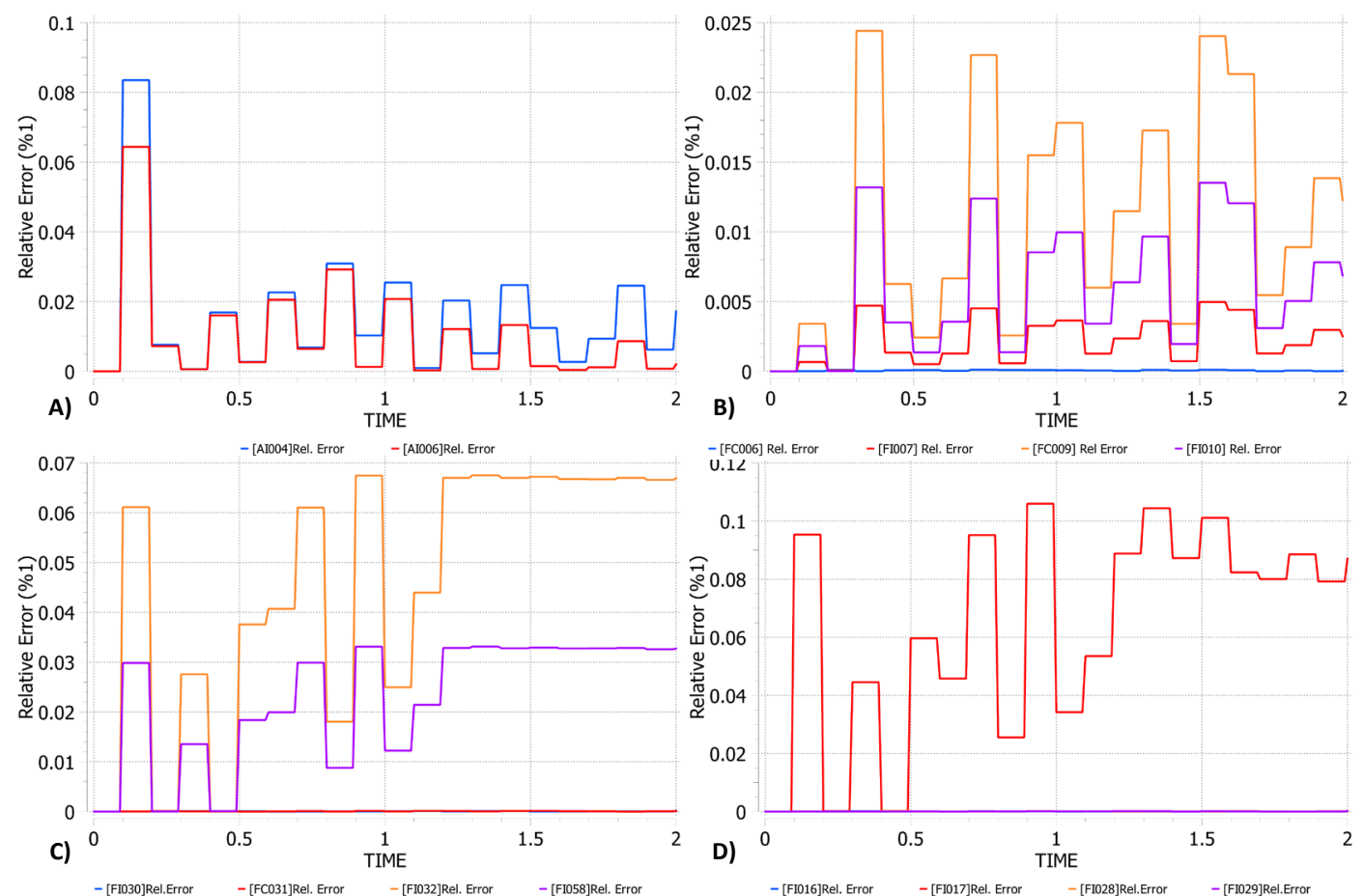
Fig. 3.14 - Case study A (G3) accuracy of parameter and state estimations. A, B, C and D, present the relative error $\left(\%^{*}\right)$ of online measurements over time, compared to the estimations computed by RTRS tool. *Estimation error over actual value in $\%$.

\subsubsection{RESULTS AND DISCUSSION}

Even though a discussion based on the results of the $\mathrm{WiA}$ is given for each scenario and case study, the focus is on the methodology and tool purpose, i.e. RTRS, rather than a detailed analysis of all process variables and constraints which is out of the scope of this study. Thereby, only the main affected variables' results are presented, in order to analyze the method potential as decision support tool for operators.

RTRS is run on a PC Intel ${ }^{\oplus}$ Core $^{\mathrm{TM}} \mathrm{i7}-6500 \mathrm{U} \mathrm{CPU}$ at $2.50 \mathrm{GHz}$ RAM $16 \mathrm{~GB}$, and takes $26 \mathrm{~s}$ and 40 s on average for Case A and B respectively, per sample time to complete the execution. In addition, costs and prices used in (3.10) are shown in Table 3.7. It is worth mentioning that, prices and costs are representative rather than real for confidentiality reasons.

Table 3.7 - Prices and costs considered for the economic valuation of scenarios (3.10).

\begin{tabular}{|c|c|c|c|}
\hline & Description & Case I & Case II \\
\hline$p r_{i}\left(\mathrm{k} € / \mathrm{m}^{3}\right)$ & $\begin{array}{l}\text { Price of HC produced of quality } i \text {, i.e.: Diesel A, Diesel } \\
\text { B and GOBA. Represents increase of profit in the } \\
\text { process economy balance (6). The same price is used } \\
\text { across HC qualities. }\end{array}$ & 1 & 1 \\
\hline$c t_{\mathrm{HS}, \mathrm{i}}\left(\mathrm{k} € / \mathrm{Nm}^{3}\right)$ & $\begin{array}{l}\text { Cost of high purity hydrogen supply of source } i \text {. } \\
\text { Represents loss of profit in the process economy balance } \\
(6) \text {. Two high purity hydrogen sources are used across } \\
\text { cases }(\mathrm{i}=1,2) \text {. }\end{array}$ & $\begin{array}{l}1.5 \mathrm{E}-3, \\
2.0 \mathrm{E}-3\end{array}$ & $\begin{array}{l}1.5 \mathrm{E}-3, \\
2.0 \mathrm{E}-3\end{array}$ \\
\hline$c t_{\mathrm{LPH}, \mathrm{i}}\left(\mathrm{k} € / \mathrm{Nm}^{3}\right)$ & $\begin{array}{l}\text { Cost of low purity hydrogen supply of source } i \text {. } \\
\text { Represents loss of profit in the process economy balance } \\
\text { (6). One source of LPH is used across cases }(\mathrm{i}=1) \text {. }\end{array}$ & $1 \mathrm{E}-4$ & $1 \mathrm{E}-4$ \\
\hline
\end{tabular}

\subsubsection{CASE I: RTRS AT PLANT LEVEL}

This case study is an example of RTRS at plant level (G3 process unit, Fig. 3.11). Baseline $(\mathrm{BL})$ process conditions represent an unexpected $15 \%$ increase in $r d_{H 2 S S}\left(\mathrm{H}_{2}\right.$ demand per volume unit of feed) at $0.2 \mathrm{~h}$ (see Fig 3.16), these change of condition is a consequence of plant feed becoming richer in sulfur components. Moreover, it impacts on 
the recycled gas purity (A1004, Fig. 3.15 - left) resulting in a significant drop of this controlled variable. Recycle gas purity lower limit (LL), $77 \% \mathrm{H}_{2}$, is violated after the change in $\mathrm{H}_{2}$ demand realizes, requiring an action from the operator. In this context, SA shows the effect of increasing the purification membrane load (FT042, Fig. 3.15 - right). SB presents the impact of using HPH make-up feed instead (FC233, Fig. 3.15 - right).

Certainly, it has to be noticed the fact that for both sets of actions profit is lower than for $\mathrm{BL}$. This is explained due to an increase in $\mathrm{H}_{2}$ demand in reactors $(R 1 R 2$, which rises make-up gas demand. Thereby, hydrotreating costs are higher producing the same amount of low sulfur product. For instance, this would be the case when crude oil tanks are changed or $\mathrm{HC}$ feed streams change their composition for any other reason.

It is seen that, although both SA and SB are effective mitigation actions, towards steering the process within recycle gas constraint (Fig. 3.15 - left), SA provides the most cost-effective response outweighing SB strategy by around 47\% (Fig. 3.16). This results show the key importance of purification units for addressing the best possible operation. It must be borne in mind that, membranes are rarely operated automatically from the control room, instead, are likely to require manual intervention from a field operator. This is exactly the case of G3 process unit. Therefore, this analysis is valuable as a training example for operators, as well, for it quantifies actual impacts of different mitigation alternatives (Fig. 3.16). In particular, presenting that is better to address $\mathrm{H}_{2}$ demand changes at plant level by manipulating the purification membrane rather than make-up gas. This results are in line with previous finding presented by Galan et al. (2018), where a focus on the optimum $\mathrm{H}_{2}$ management policy is addressed showing the importance of membranes operation. 


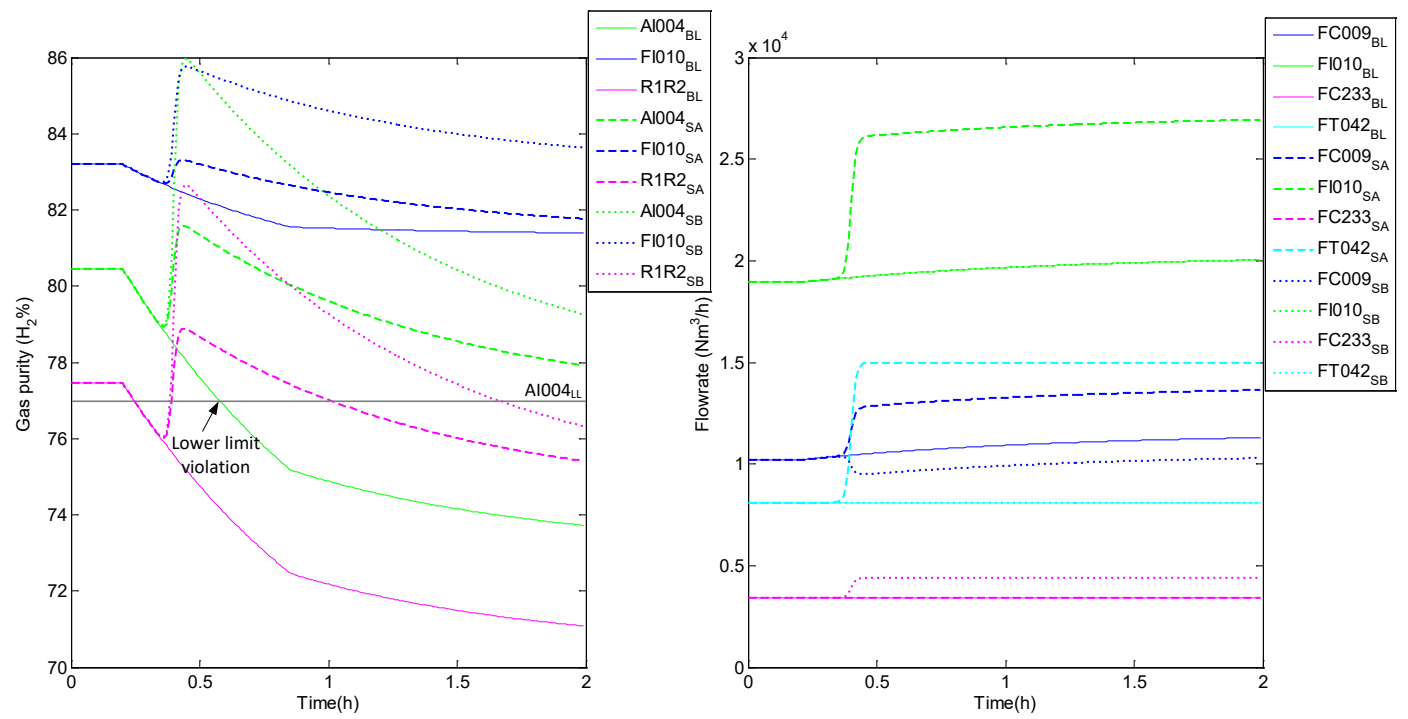

Fig. 3.15 - G3 main variables computed at BL, SA and SB. Left, gas purities of streams: recycle gas (AI004), aggregated make-up and purified gas (FIO10) and reactor effluent (R1R2). Lower limit violation is pointed with an arrow $\left(A 1004_{L L}\right)$. Right, gas flowrates of streams: $\mathrm{LPH}$ make-up gas (FCO09), aggregated make-up and purified gas (FI010), HPH make-up gas (FC233) and membrane feed (FT042). 


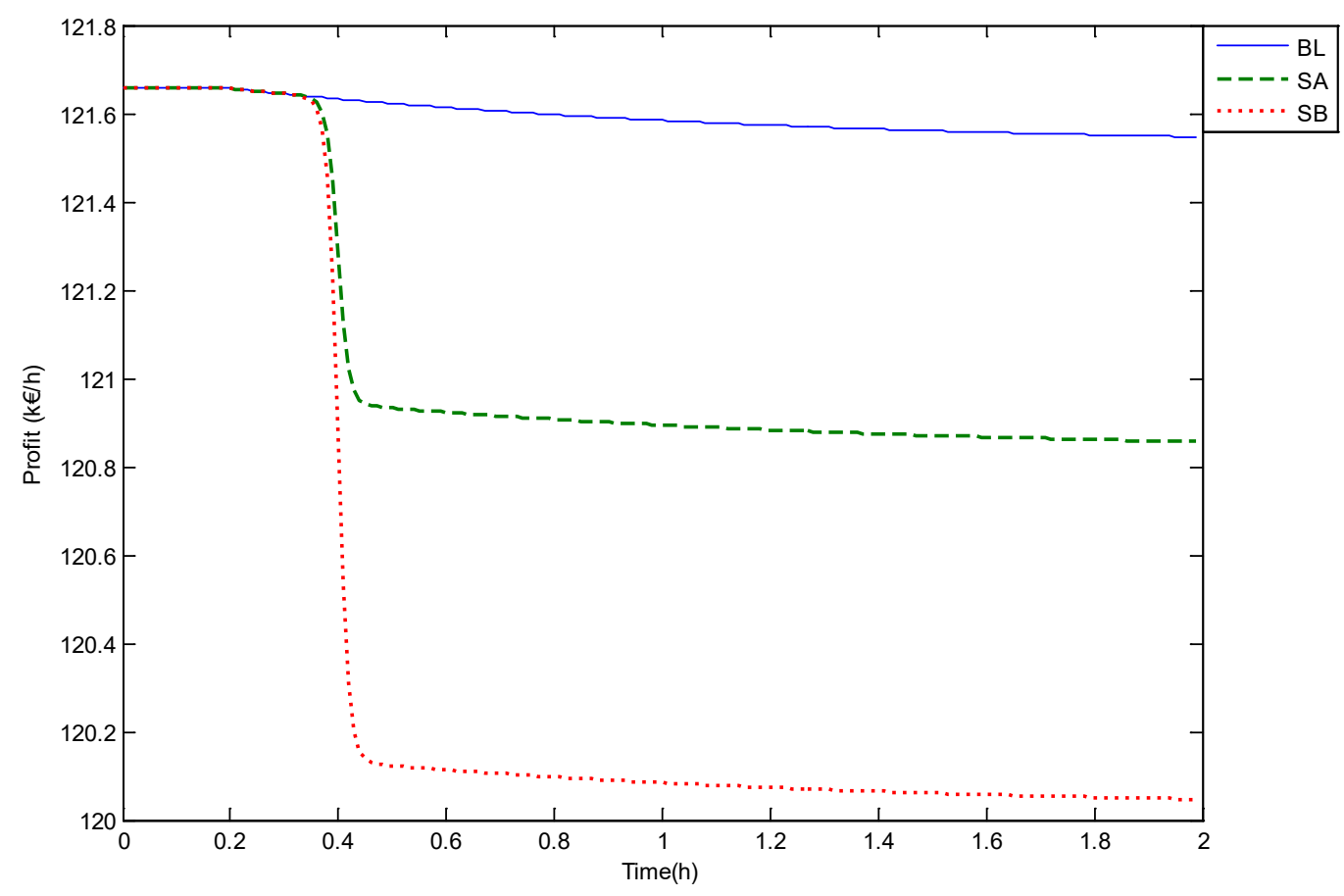

Fig. 3.16 - G3 process profit, in $\mathrm{k} € / \mathrm{h}$ at all scenarios. Profit does not consider operational constraints (3.10), such as gas purity in reactors. Values are representative rather than actual for confidentiality reasons.

\subsubsection{CASE II: RTRS AT NETWORK LEVEL}

This case study shows an example of RTRS at network level. BL, presents a $\mathrm{H}_{2}$ demand increase due to a HC load rise by $5 \mathrm{~m}^{3} / \mathrm{h}$ (3.2\%) in HDS1 (FCO01, Fig. 3.17 - right), decreasing recycle gas purity of HDS1 below its lower limit (see Al001 in Fig. 3.18 - right), $68 \%\left(A / O 01_{L L}\right)$, requiring an action from the operator. In this context, SA shows the effect of manipulating HPH make-up (FIOO3, Fig. 3.17 - left) and FCOO1, being both mitigation actions local to HDS1. On the other hand, SB shows corrective actions manipulating HPH make-up in HDS1 and HDS2 (FIOO4, Fig. 3.17 - left), being a network-wide strategy.

In this case, $\mathrm{SA}$ and SB satisfy the recycle gas purity constraint after time $0.2 \mathrm{~h}$, therefore are both deemed as corrective actions (Fig. 3.18 - right). However, it is observed a significant negative impact on Profit of SA compared to SB (Fig. 3.19 - right). The reason for this is that $\mathrm{SB}$ copes with $\mathrm{HC}$ load changes by purely rearranging $\mathrm{H}_{2}$ distribution across 
the network. Concretely, cutting back $\mathrm{H}_{2}$ feeding HDS2, allowing more make-up to HDS1 (Fig. 3.17 - left). Therefore, impact on process economy is minimized in the sense that production is not reduced. However, SA strategy applies only HDS1 local MVs to mitigate the change of condition, taking a toll on process economy. Moreover, it does not reach the production level of $160 \mathrm{~m}^{3} / \mathrm{h}$ as required (FCO01, Fig. 3.17 - right). An interesting outcome to point out is that, an operator would rather manipulate variables within his process unit boundaries, not considering the impact of actions in other process units in the network. In this case, it is clear how that approach is not the best possible, presenting an alternative that enables an increased HC load while keeping constraints within limits. Even more, this is an example of how network interactions between plants are key elements for troubleshooting. And RTRS demonstrates its usefulness for addressing this sort of challenges.

It must be pointed out that building models at network level is more complex than at plant level. Similarly, the analysis of results and their interpretation requires more knowledge. Thereby, it is sensible to assume that RTRS at network level should be a useful tool for experienced operators or supervisor, while might seem not easy to build up meaningful sets of actions or scenarios for novice operators. Still, for training purposes it should be valuable as well. 


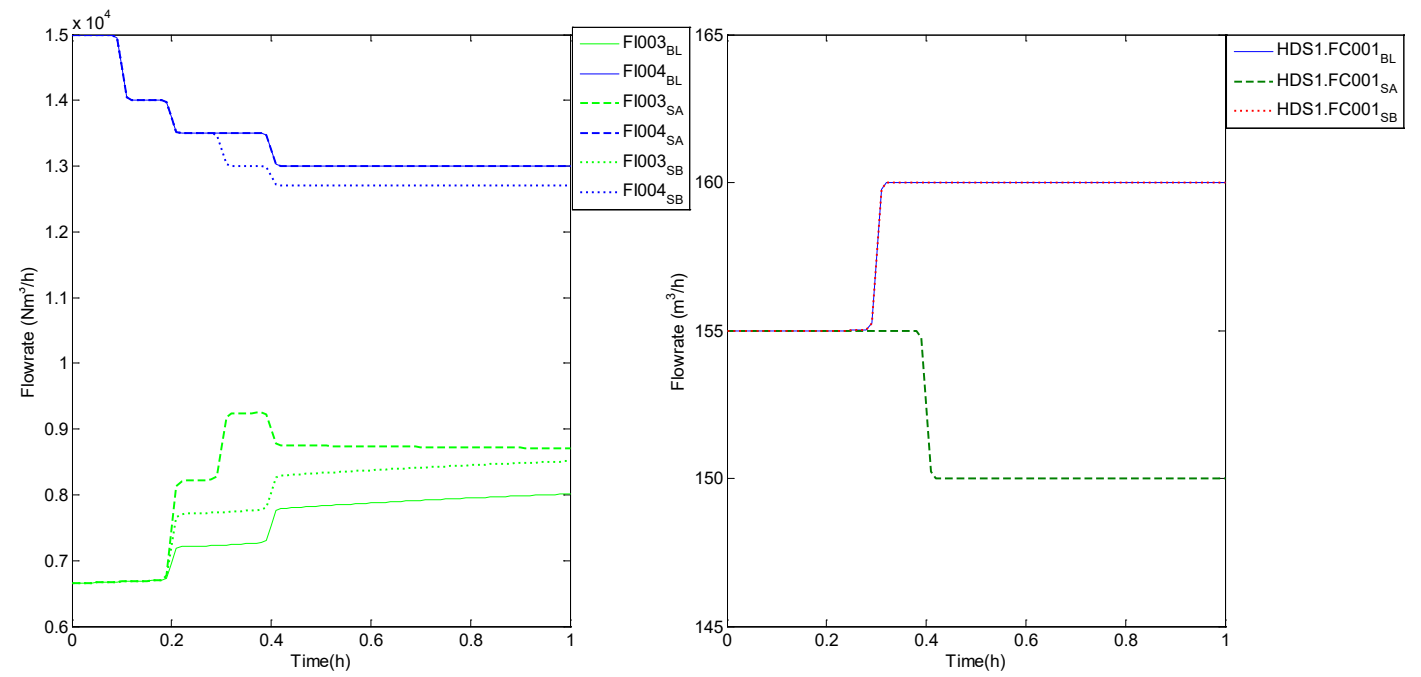

Fig. 3.17 - Left: HPH make-up gas streams FIOO3 (HDS1) and FIOO4 (HDS2) in Nm³/h. Right: HDS1 hydrocarbon load (FCOO1) in $\mathrm{m}^{3} / \mathrm{h}$.

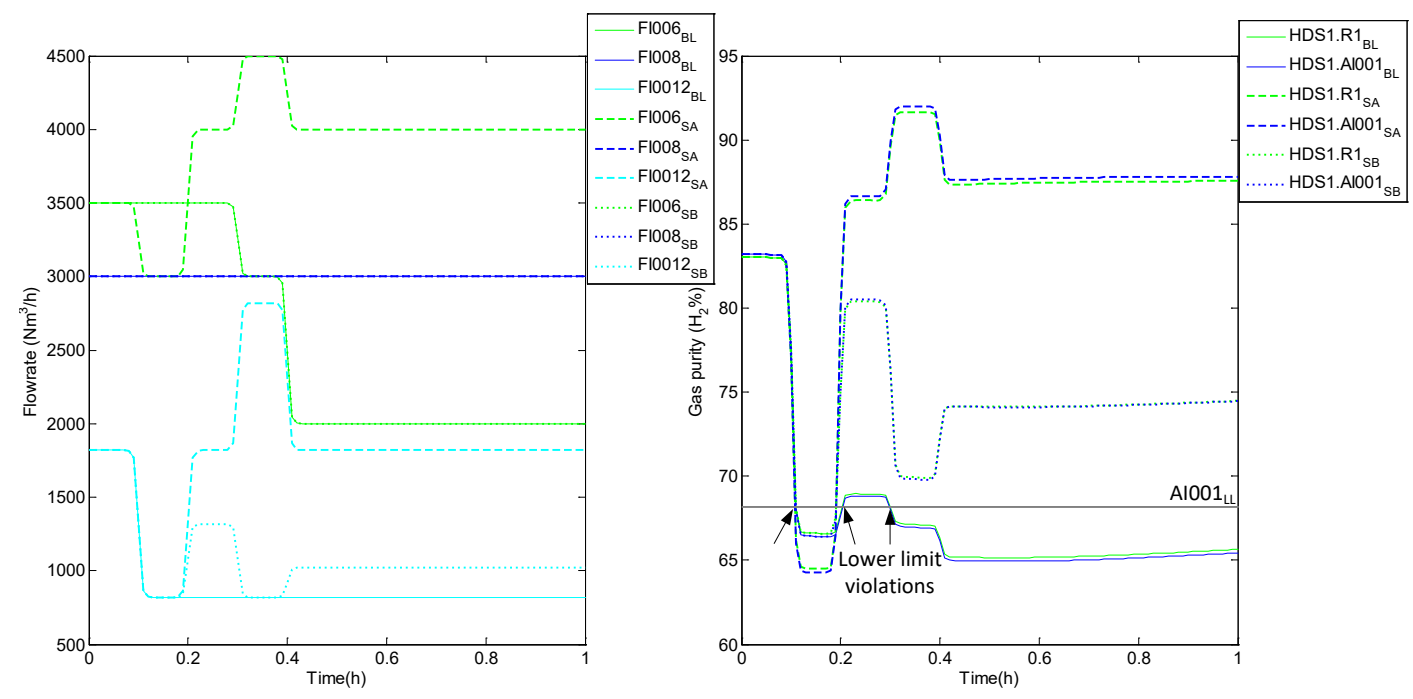

Fig. 3.18 - Left: LPH streams in $\mathrm{Nm}^{3} / \mathrm{h}$. Right: HDS1 recycle (AIO01) and reactor effluent (R1) gas purities in $\mathrm{H}_{2} \%$. Lower limit violations are pointed with arrows $\left(A / 001_{L L}\right)$. 


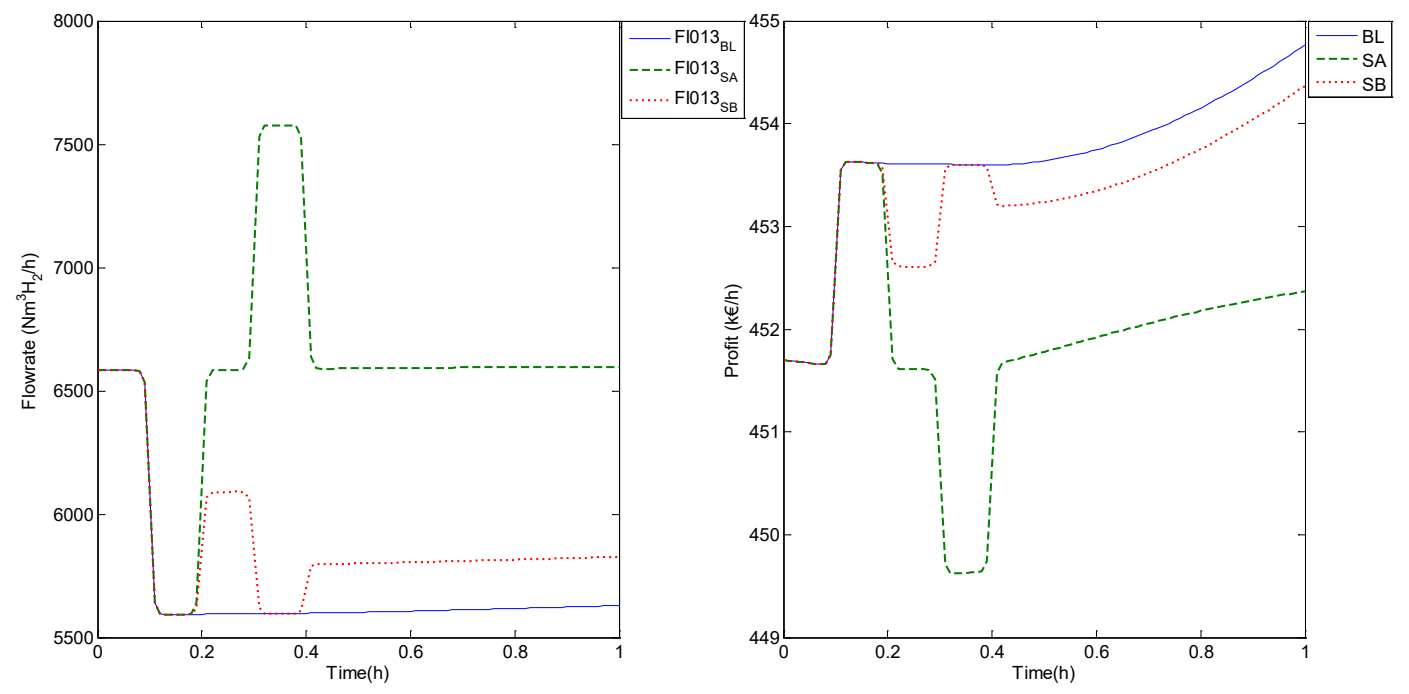

Fig. 3.19 - Left: gas flowrate to FGH (FIO13) in Nm3 H2 /h. Right: process profit in k€/h (3.10).

\subsubsection{GENERAL HIGHLIGHTS}

RTRS methodology has significant versatility, due to its capability of adaptation to several purposes such as analysis based on predefined sets of actions or training. For instance, this work showcases WiA based on RTRS at two levels in a process network (plant level and network level). Moreover, RTRS presents especial interest as a decision support tool for analyzing mitigation actions due to change of condition. Additionally, control room schematics can be replicated on the RTRS graphic interface for ease of understanding and effective communication of outcomes throughout the organization.

Other favorable accountability of RTRS is its complementarity to model predictive control (MPC) and real-time optimization (RTO) layers. Particularly if those are considering the same process system, for example in a refinery $\mathrm{H}_{2}$ network the integration would be MPC, RTO and RTRS. MPC and RTO would take care of actual closed-loop control, while RTRS may contribute providing an analysis tool for better understanding and testing of RTO and MPC solutions. Thereby, it might be the case where operators come up with a set of actions with similar impact on profits than RTO, though easier to perform due to model limitations for representing all constraints and casualties. In this dimension, RTRS complements other existing decision-making and control tools in the process industry. 
However, this aspect requires further exploration, in order to solve interactions of the different tools with ease. Being the ultimate, ideal, aim to efficiently integrate digital information from: process models, business indicators, maintenance monitoring and supply chain, into a decision support framework.

Another advantage of RTRS is its intuitiveness, since is based on well-known techniques (MHE and DAEs computation). Hence, is relatively easy to replicate and develop for any given dynamic process system. Nonetheless, it could be challenging for systems with complex dynamics, if a previous appropriate components library is not available.

In spite of all the previous comments, there are RTRS current limitations to point out as well. Firstly, it is duly noted that single shooting optimization approach has limited effectiveness for handling path constraints or unstable solutions, and generally speaking is not the state of the art in dynamic optimization techniques. This shortcoming should be considered in future work, exploring strategies for automatic finite elements orthogonal collocation and differentiation within a simulation framework. Secondly, RTRS itself is unlikely to provide optimum solutions and run in open-loop, which is generally speaking a downside that comes alongside and at the cost of versatility. However, pairing RTRS with RTO / MPC applications should partly compensate this aspect as mentioned before.

In addition, RTRS may still face usability issues depending on how well trained are end-users towards full potential utilization of the tool, especially on fundamentals of simulation, modeling and optimization. In this respect, the graphical user interface (GUI) plays a critical role in making RTRS usable as seamlessly as possible across the business. In fact, it has been suggested several times in literature that apart from technical hurdles there are workforce hurdles, essentially due to shortage of optimization and simulation expertize at many levels in the process industry (Cameron et al., 2019; Ignacio E Grossmann and Harjunkoski, 2019; Harjunkoski, 2016; Harjunkoski et al., 2014). Even the simplest optimization-based applications require fundamental knowledge on optimization formulation, modeling and mathematical programming, which is sometimes overlooked 
by end-users and practitioners. Thereby, successful deployments of industrial applications may be heavily dependent on the support of experts (e.g. PhD), however, these are most likely, neither a local resource nor available to maintain the application in the long term (Ignacio E Grossmann and Harjunkoski, 2019; Harjunkoski, 2016). As a consequence, successfully deployed tools risk reaching their full potential if not looked after by teams with the right qualifications and training. Impact of optimization related skills shortage in the long term are not clear, nevertheless, the process systems engineering community generally agrees that it is indeed a non-negligible factor when considering wide and open utilization of optimization-based application across the industrial sector.

\subsection{CONCLUSIONS}

A real-time reconciled simulation tool is presented, aimed at aiding process network operators in their decision-making process, especially under changing plant conditions. RTRS effectively combines dynamic state estimation techniques (MHE) into a simulation environment, which enables both: online data pulling from plant information systems and input of manipulated variables for assessing future conditions. In this context, RTRS is a general concept and virtually applicable to any process network supported on a FPM, given a dynamic library of components is available.

In order to evaluate the usefulness of RTRS as a decision support tool for operators, a what-if analysis approach is conducted on two case studies: an individual process unit (case I) and a process network (case II). Case study I, consists in a real plant of Petronor refinery, studied for a baseline condition where hydrogen demand increases by $15 \%$ unexpectedly. Two corrective sets of actions are considered for mitigation (SA and SB) and assessed over their economic impact. It is demonstrated that SA is advantageous over SB, due to a more efficient use of hydrogen purification membranes available in G3. In addition, state and parameter estimation results are presented for case study I, showing a satisfactory accuracy for all key instruments. Case study II, consists in a representative hydrogen network, studied for baseline condition where one process unit faces an 
increase in hydrogen demand due to an ramp-up in hydrocarbon load. It is shown that set of actions SB is a better mitigation action, due to a more efficient hydrogen management across the network rather than at plant level.

Generically speaking, RTRS methodology is promising for: its versatility of application and ease of communication, complementarity with current plant-wide control systems (RTO / MPC) and intuitiveness of implementation. On the contrary, absence of optimal mitigation actions guarantee and single shooting sequential optimization, are identified as promising research areas for enhancing RTRS features going forward.

Future research directions are discussed, highlighting the importance of RTRS enterprise-wide integration with other modular decision support tools. Enabling fully interconnected information at all levels, is likely to bring added value to everyone involved and a step forward in the path towards a wisely applied digital twin approach. 
4 OPTIMIZATION UNDER UNCERTAINTY 



\section{Abstract}

This section describes the problems associated with the implementation of a realtime optimization (RTO) decision support tool, for the operation of a large scale hydrogen network of an oil refinery. In addition, a formulation which takes into account the stochastic uncertainty of hydrogen demand, due to hydrocarbons quality change, is described and further studied, focusing on its utility in the decision-making process of operators. An integrated robust data reconciliation, and economic optimization, considering plant-wide uncertain parameters is presented and discussed. Moreover, stochastic uncertainty in hydrogen demand is assessed for its inclusion within the RTO framework. A novel approach of the decisions stages at hydrogen producers and consumers is proposed, which supports the formulation of the problem as a two-stage stochastic non-linear program. Representative results are presented and discussed, aimed at assessing the potential impact in the hydrogen management policies. For this purpose, the value of the stochastic solution, perfect information, and expectation of the expected value are analyzed. Complementarily, a risk-averse formulation is presented (value-at-risk and conditional-value-at-risk) and its results compared against the formulation without risk considerations. Finally, some attention is given to future directions of this decision support tool, based on these work contributions, including the importance of the decision makers' participation in the analysis of the potential impact of risk-averse results. 



\subsection{INTRODUCTION}

Optimization techniques in process control are critical to cope with tight specifications while keeping production sites running at cost-efficient rates with optimum efficiency. In this regard, real-time optimization (RTO) is a key player that enables plants to actually operate at its economic optimal points in real-time. Moreover, RTO applications have different ways of considering uncertainty associated to real-time operation of plants, such as structural uncertainty (see section 1.2.2 Modifier adaptation and the references cited therein), measurement errors (see section 1.2.1 Data reconciliation and parameter estimation and the references cited therein) and unknowns that realize at a later stage (see section 1.2.4.1 Stochastic programming and the references cited therein). In this chapter I study and analyze the incorporation of stochastic programming into an actual crude oil refinery hydrogen network RTO, considering risk-neutral and risk-averse economic objectives.

The RTO standard architecture presented in Fig. 4.1 - top (note this is an expansion of Fig. 1.4), with an RTO layer that uses nonlinear steady-state models to generate fix targets for the MPC for periods of the order of hours, does not manage properly the dynamic aspects above mentioned. Alternatively, the RTO and MPC layers can be combined in an economic MPC (EMPC), see Fig. 4.1 - bottom, or optimal dynamic operation problem as described in Engell (2007) and Gonzalez Santos et al. (2001). This approach solves the inconsistency problem between layers that may appear due to the use of different models in RTO and MPC, and it is well stablished for continuous processes, but requires solving large-scale dynamic optimization problems in long computation times in order to allow for real-time implementation, which may be a significant obstacle for its implementation. For a review on RTO, MPC and their integration refer to Appendix C and the references cited therein. 

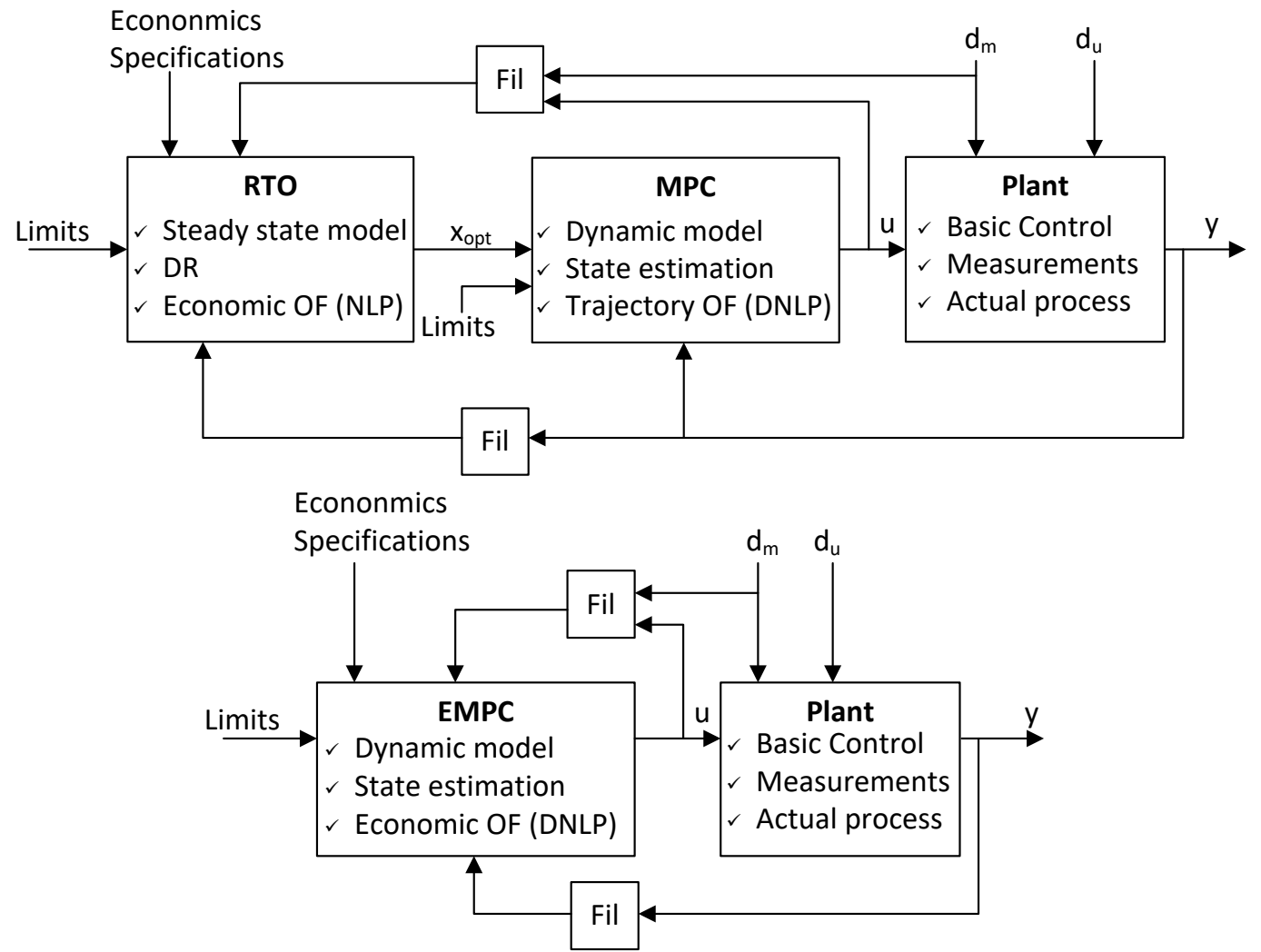

Fig. 4.1 - Simplified RTO-MPC block diagram (top). Simplified economic MPC (EMPC) block diagram (bottom). Fil: filtering of signal. $d_{m}$ : measured disturbances. $d_{u}$ : unmeasured disturbances. $u$ : input variables. $y$ : output variables. $x_{\text {opt }}$ : RTO optimal decisions that are passed on to MPC. NLP: nonlinear program. DNLP: dynamic nonlinear program. OF: objective function.

Alternatively, this chapter proposes another way of considering the joint operation of large-scale RTO with MPC, and illustrates the methodology in a case study corresponding to the hydrogen network of an oil refinery involving the joint operation of 18 plants, first introduced by de Prada et al. (2017), discussing its implementation and results. In addition, uncertainty in the hydrogen demand is incorporated in the nonlinear optimization problem as an extension of the deterministic RTO (with and without risk considerations), analyzed and compared against deterministic results. Furthermore, particular attention is given to discussing the advantages and shortcomings of the twostage stochastic formulations presented, i.e. risk-neutral and risk-averse. 


\subsubsection{HYDROGEN NETWORK CASE STUDY}

Hereinafter the fundamental characteristics of the case study hydrogen network are revisited. For a general overview on hydrogen networks refer to section 1.3 Hydrogen networks of crude oil refineries and the references cited therein.

In the particular the hydrogen network of the refinery under consideration, Petronor (Petronor, 2020) in Muskiz, Spain, involves 18 plants: two producers of fresh hydrogen, two platformer plants and 14 consumer plants, most of them hydrodesulfurizers connected by means of several headers that operate at different pressures and hydrogen purities as in Fig. 4.2 (repeats diagram in Fig. 3.8 - Bottom). Note that a consumer plant can be fed from different sources.

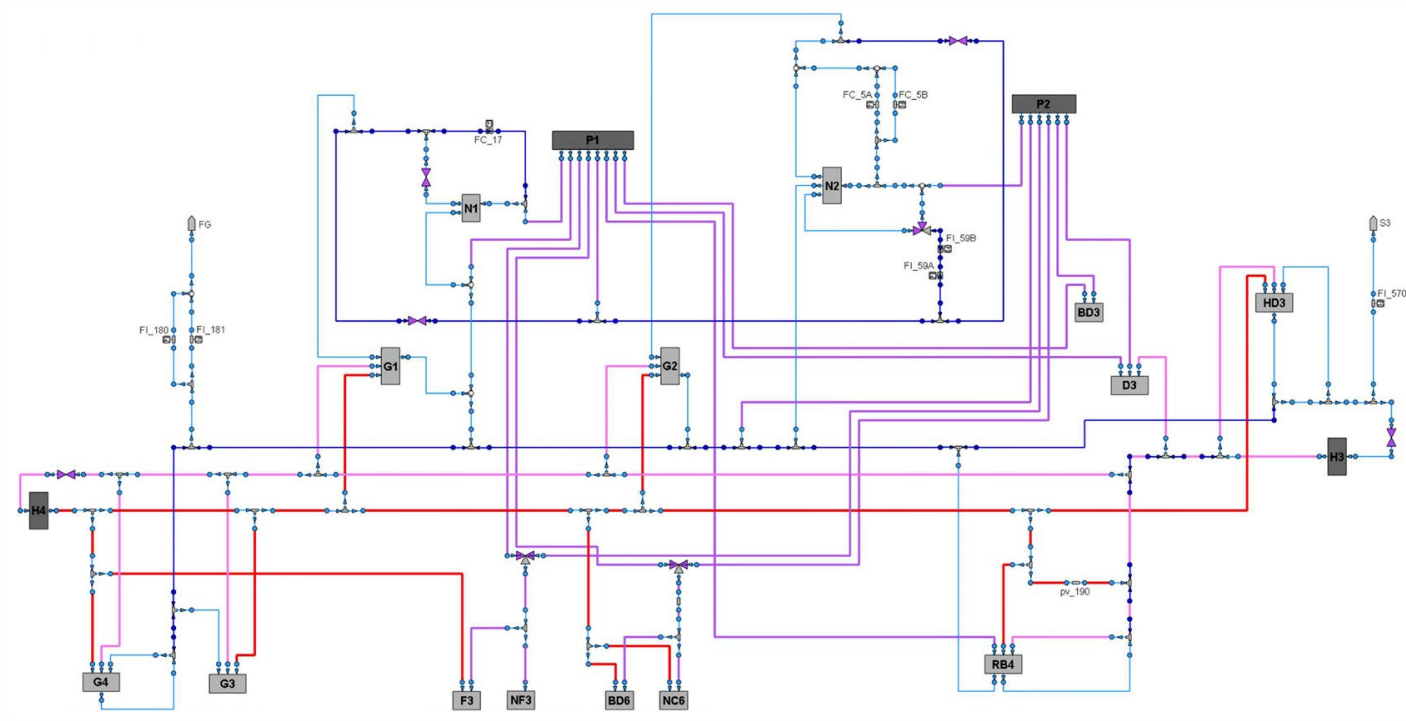

Fig. 4.2 - Schematic of the hydrogen network with producer (grey boxes) and consumer (light grey boxes) plants connected by several headers, among them H4 (red), H3 (light purple) and LPH (blue). Source: Gómez Sayalero (2016).

A simplified schematic of a typical hydrodesulfurization plant can be seen in Fig. 4.3, which is more detailed than Fig. 3.9 of chapter 3. Similarly, hereinafter I revisit the main processes occurring in hydrotreating units in crude oil refineries, already described in chapter 3 , for the sake of clarity. 
The high sulfur hydrocarbon $(\mathrm{HC})$ feed is mixed with treatment gas (typically around $85-90 \%$ hydrogen content, and high pressure) coming from different sources (high and low purity headers, HPH and LPH respectively). This cold mixed stream is heated to reaction temperature, around $300-350 \circ \mathrm{C}$, by heat exchangers and a furnace (reactor load heating subsystem, RLHS) before going into the reactor. This untreated hot stream reacts on the catalyst fixed bed of the reactor, where the actual desulfurization and other side reactions take place. Due to the exothermic nature of these reactions, the outlet stream is used to preheat the cold stream load in the heat exchangers within the RLHS. The next stage of the process is the separation of gas and liquid, for this purpose the high pressure separator (HPS) is fed with the cooled reactor outlet, and produces two outlets:

- High pressure sour gas (rich in hydrogen sulfide, $\mathrm{H}_{2} \mathrm{~S}$ ),

- Medium or low pressure mixed gas and HC.

The mixed stream is routed to the low pressure separator (LPS) where flashed offgas is sent to the low pressure gas absorber and oil phase routed to the stripper for stabilization. In the stripper the last traces of dissolved gas are removed so that the bottom product meets specifications, and side cuts routed to other process units as feedstock components. Stripper off-gas typically goes to fuel gas (FG) after some gas treating aimed at absorbing hydrogen sulphide $\left(\mathrm{H}_{2} \mathrm{~S}\right)$ in the low pressure absorber. The liquid outlet (treated $\mathrm{HC}$ ) at this stage is ready to be cooled and dried to be pumped into the blending system or stored in tanks.

In the high pressure circuit (see Fig. 4.3), gas is recycled back through a compressor, after being sweetened in a gas absorber (hydrogen sulphide removal). In addition, high pressure gas can be fed into a permeation membrane to purify its hydrogen $\left(\mathrm{H}_{2}\right)$ content, or purged to the LPH (circa 5\% less than treatment gas, e.g.: 75-85\%). 


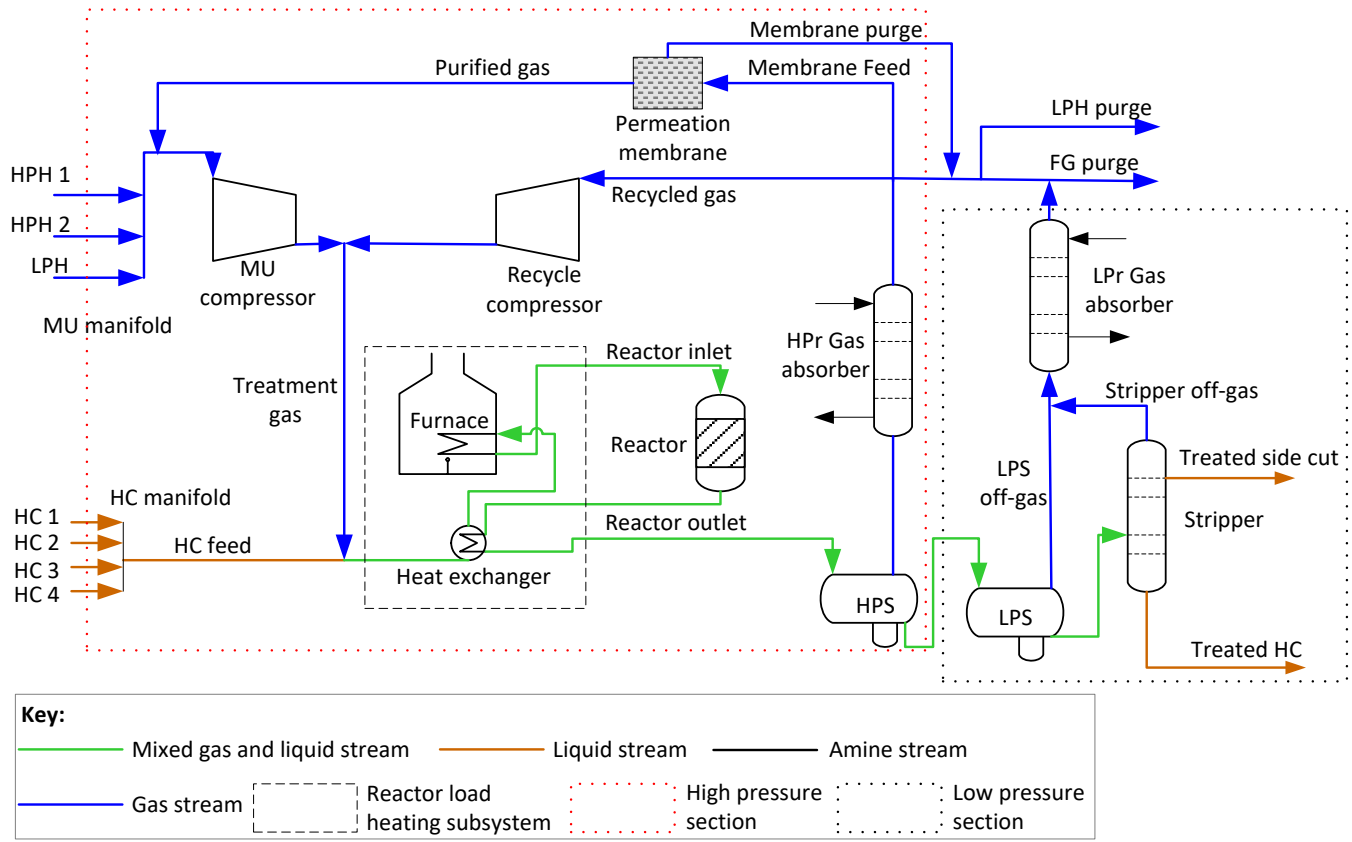

Fig. 4.3 - Simplified schematic of a generic hydrodesulfurization (HDS) plant. HPH: High purity gas header. LPH: Low purity gas header. HC: Liquid hydrocarbon; MU: Make-up. FG: Fuel gas. HPS: high pressure gas/HC separator. LPS: Low pressure gas/HC separator. HPr: high pressure. LPr: Low pressure.

One important aspect of the operation is the fact that preserving catalyst lifecycle requires hydrogen excess at all times, regardless of the demand. Since hydrogen is a product that is very difficult to store and the plants have variable hydrogen demand according to the type and flow of the hydrocarbons being processed, the producer plants always generate more hydrogen than what is consumed in order to guarantee that enough hydrogen is available under any circumstance. This policy is aimed at protecting catalysts, which are not only an expensive material but also require a plant shutdown to be replaced. Thereby, minimum $\mathrm{H}_{2}$ purity figures are operational constraints, subjected to change over time (e.g.: start of run, or end of run) mainly due to catalyst and load quality conditions. All excess gases across the network end up in the fuel gas header (basic pressure control at plant level), which complemented with natural gas and liquid petroleum gas (LPG) is used to fuel the gas burners plant-wide. As hydrogen is expensive 
to produce, a good management of the network should coordinate the operation of all plants, matching demand and production in order to minimize losses of hydrogen to FG.

Another key point related to the operation of the reactors is purity management. As mentioned before, the gas recycled from the separation units (HPS) has a lower purity than the treatment gas fed to the reactor, but its purity can be increased using permeation membranes or, after being sent to $\mathrm{LPH}$, reused in other plants either directly or mixed with fresh hydrogen to increase its purity. As a result, the hydrogen network operates with several headers at different purities and pressures as represented in the simplified schematic shown in Fig. 4.4, which displays two producer units with their corresponding headers, supplying high purity (HP) hydrogen to $N$ consumer plants that deliver or consume (low purity) recycled gas from the LPH, and may also send low purity gas excess to the FG network by means of a local pressure controller (PC) which sends a control signal to an automatic valve (AV).

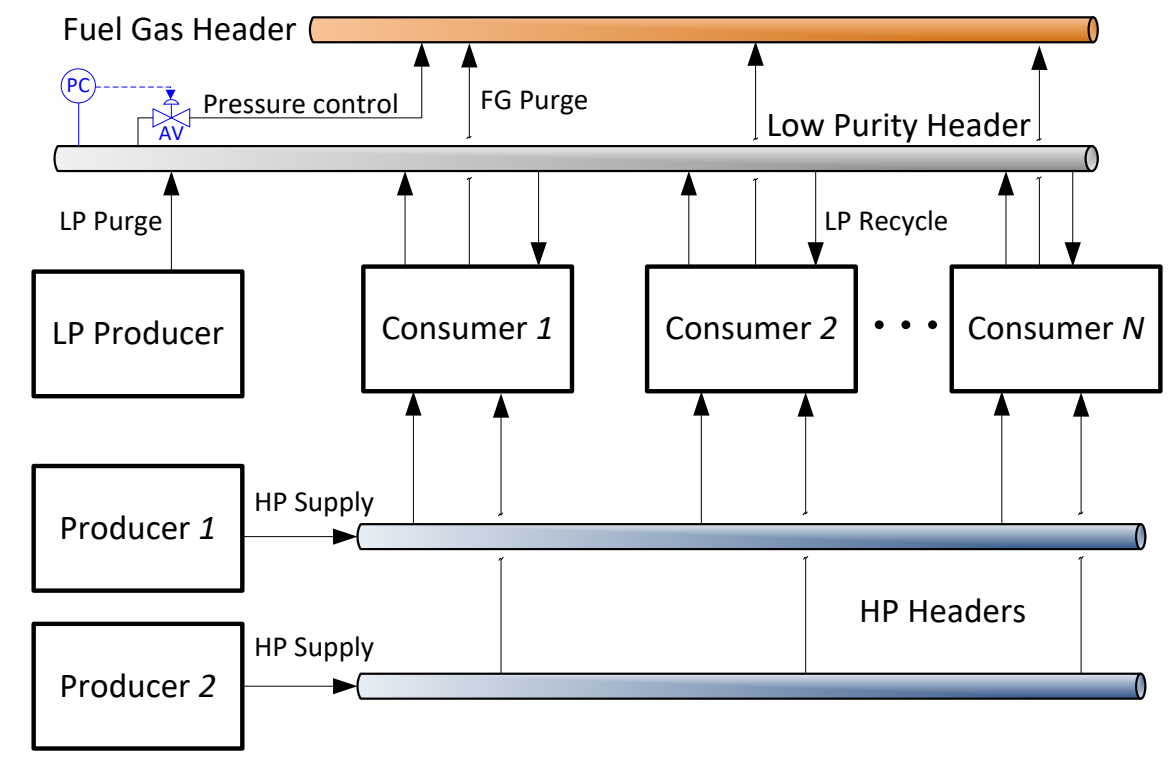

Fig. 4.4 - Schematic showing the different types of headers found in hydrogen network: high purity headers (blue), low purity header (grey), fuel gas header (brown). An automatic valve (AV) controls the pressure of the low purity header (LPH) by controlling the flow from the LPH to the fuel gas header (FGH). PC: pressure controller. AV: automatic valve. 
Proper management of the network requires deciding in real-time, according to the hydrogen demands from the reactors and variable hydrogen flows generated by the platformer plants, how much fresh hydrogen should be produced by each producer plant, and how to distribute the hydrogen through the network and internally in the consumer plants so that the losses to FG, or in general costs, are minimized. In addition, the operation of the network has to consider as the most important economic target the maximization of the hydrocarbon loads processed in the hydrodesulfurization plants, which may be limited by the hydrogen available and the production aims stablished by the planning of the refinery for the period under consideration. Notice that reducing losses of hydrogen to FG may increase the hydrocarbon processing if hydrogen is the limiting factor, which provides additional value to the optimal management of the network. Furthermore, optimal decisions must satisfy all process constrains imposed by the equipment, operation, safety, targets or quality.

\subsubsection{OUTLINE}

The remaining of this chapter is organized as follows. After the introduction, section 4.2 describes the models and formulation of the optimization problem. In addition, presents the architecture of the system implemented in the refinery and discusses the fundamental results obtained with the deterministic RTO framework. Next, sections 4.3 to 4.7 are devoted to describe, formulate and discuss the stochastic problem considering two alternative aims of optimization under uncertainty, risk-neutral and riskaverse. The chapter ends with conclusions and future work suggestions.

\subsection{HYDROGEN NETWORK OPTIMIZATION FRAMEWORK}

Hereinafter I present the hydrogen network models used in the in the deterministic optimization problem and discuss the system architecture developed to effectively implement the network-wide real-time optimization (RTO). This RTO provides the deterministic model utilized as starting point for developing a two-stage stochastic model for implicit consideration of hydrogen demand uncertainty, risk-neutral and risk-averse, 
which is the core contribution of this chapter and is discussed from section 4.3 Incorporation of explicit uncertainty onwards.

\subsubsection{MODELS AND DATA RECONCILIATION}

Optimization of complex systems requires proper network and plant models validated against process data. One of the main obstacles in developing these models is the lack of reliable information about many streams and compositions besides the nature of hydrogen. Most of the hydrogen flow measurements are volumetric thus these must be compensated using pressure, temperature and molecular weight of the stream to obtain mass flows. Nevertheless, hydrogen purity measurements are not always available and, even when measured, the molecular weight of the stream is most likely either unknown or uncertain. This is due to the fact that the gas stream contains impurities (undesired light $\mathrm{HC}$ of various sources) of unknown and changing molecular weight much larger than the one of hydrogen (mainly methane and ethane), which is only $2(\mathrm{~g} / \mathrm{mol})$. For example, a stream with purity $90 \%$, where one half of the impurities change composition, for instance from methane to propane, can change the molecular weight of the stream in $41 \%$. Notice that besides flows and compositions, other important variables, such as hydrogen demand in the reactors, are not measured and change over time with the composition of hydrocarbons being processed.

This means that, before any optimization can be performed, a procedure to obtain reliable information from the plant using the plant measurements should be implemented. Data reconciliation can be used for this purpose as it offers a way of estimating the values of all variables and model parameters coherent with a process model and as close as possible to the measurements. Data reconciliation is formulated as a large optimization problem searching for the values of variables and parameters that satisfy the model equations and constraints and that, simultaneously, minimize a function of the deviations $(e)$ between model and measurements, properly normalized. 
In this case study, a steady state first principle model of the hydrogen network was available from previous works by Sarabia et al. (2012), and Gómez Sayalero (2016). This model is based on the same basic principles used in the dynamic simulation library (see chapter 2), applied to a steady state system. In essence, the model comprises mass balances of hydrogen $\left(\mathrm{H}_{2}\right)$ and light ends (considered altogether as a single pseudocomponent, $L I G)$ at all nodes $(N)$ of the network as in the pipelines, headers, and units as in (4.1.a-d), where $F$ stands for gas flows, $X$ are hydrogen purities, and $M W$ refers to molecular weights. Each $k$ node has outlets $i$ and inlets $j$ streams.

In addition, the model incorporates other first principle and reduced order equations for reactors (4.2.a-e), membranes (4.3.a-e), separation units (4.4.a-f), compressors and headers (4.1.a-d). Reduced order models are used for permeation membranes fitting their parameters to historical plant data to determine explicitly (4.3.a), following previous works methodologies (Galan et al., 2018; Gómez Sayalero, 2016). Table 4.1 presents a description of all variable and subscripts, while engineering units used in this chapter are provided in Table 4.2.

At all nodes $N$ within the network:

$$
\begin{aligned}
& \sum_{i, \text { out }} F_{k, i}=\sum_{j, \text { in }} F_{k, j} \quad \forall k \in N \\
& X_{H 2, k} \cdot \sum_{i, \text { out }} F_{k, i}=\sum_{j, \text { in }} X_{H 2, k, j} \cdot F_{k, j} \quad \forall k \in N \\
& M W_{k} \cdot \sum_{i, \text { out }} F_{k, i}=\sum_{j, \text { in }} M W_{k, j} \cdot F_{k, j} \quad \forall k \in N \\
& 100 \cdot M W_{k}=M W_{H 2} \cdot X_{H 2, k}+\left(100-X_{H 2, k}\right) \cdot M W_{L I G, k} \quad \forall k \in N
\end{aligned}
$$

At all reactors $r$ within the network: 


$$
\begin{aligned}
& F_{\text {in }, r}=F_{\text {out }, r}+R D_{H 2, r}-R D_{L I G, r} \\
& F_{\text {in }, r} \frac{X_{\text {in }, r, H 2}}{100}=F_{\text {out }, r} \frac{X_{\text {out }, r, H 2}}{100}+R D_{H 2, r} \\
& F_{\text {in, } r} M W_{\text {in }, r}=F_{\text {out }, r} M W_{\text {out }, r}+R D_{H 2, r} M W_{H 2}-R D_{L I G, r} M W_{L I G, r} \\
& R D_{H 2, r}=H C_{\text {in }, r} \cdot r d_{H 2, r} \\
& R D_{L I G, r}=H C_{\text {in }, r} \cdot r d_{L I G, r}
\end{aligned}
$$

$\forall r \in$ Reactors

At all permeation membranes $z$ within the network:

$$
F_{\text {in, } z}
$$

$$
F_{i n, z} \frac{X_{i n, z, H 2}}{100}=F_{o, z} \frac{X_{o, z, H 2}}{100}+F_{p g, z} \frac{X_{p g, z, H 2}}{100}
$$

$$
F_{i n, z} M W_{i n, z}=F_{o, z} M W_{o, z}+F_{p g, z} M W_{p g, z}
$$

$X_{o, z, H 2}=a \frac{F_{p g, z}}{F_{i n, z}}+b \cdot X_{i n, z, H 2}+c$

$$
M W_{i n, z, L I G}=M W_{o, z, L I G}
$$

\section{$\forall z \in$ Permeation membranes}

At all separators (high and low pressure) se within the network:

$$
k_{s o l} l_{\text {se,gasHC }}=\frac{22.415 \cdot G_{\text {out }, \text { se }}\left(Y_{\text {out }, \text { se }, H 2}+Y_{\text {out }, \text { se }, \text { LIG }}\right)}{G_{\text {out }, s e} Y_{\text {out }, s e, H C}\left(\frac{M W_{H C}}{\rho_{H C}}\right)}
$$




$$
\begin{aligned}
& k_{s o l} l_{s e, H 2 L I G}=\frac{X_{\text {out }, \text { se }, H 2}}{Y_{\text {out }, \text { se }, H 2}} \cdot \frac{Y_{\text {out }, \text { se }, L I G}}{X_{\text {out }, \text { se }, \text { LIG }}} \\
& k_{s o l} l_{s e, M W L I G}=\frac{M W_{\text {out }, \text { se }, L I G}}{M W_{\text {Gout }, \text { se }, \text { LIG }}} \\
& G_{\text {in, se }}\left(100-Y_{\text {in,HC }}\right)=F_{\text {out }, \text { se }}+G_{\text {out }, \text { se }}\left(100-Y_{\text {out }, s e, H C}\right) \\
& G_{\text {in }, s e} Y_{\text {in }, \mathrm{se}, \mathrm{H} 2}=F_{\text {out }, \mathrm{se}} X_{\text {out }, \mathrm{se}, \mathrm{H} 2}+G_{\text {out }, \mathrm{se}} Y_{\text {out }, \mathrm{se}, \mathrm{H} 2} \\
& G_{\text {in, se }}\left(100-Y_{\text {in,HC }}\right) M W_{\text {Gin,se }} \\
& =F_{\text {out }, \text { se }} M W_{\text {out }, \text { se }}+G_{\text {out }, \text { se }}\left(100-Y_{\text {out }, \text { se }, H C}\right) M W_{\text {Gout }, \text { se }}
\end{aligned}
$$

\begin{tabular}{|c|c|}
\hline$F$ & Gas flow, in $\mathrm{Nm}^{3} / \mathrm{h}$ \\
\hline$x$ & $\mathrm{H}_{2}$ or LIG fraction in a gas stream, in \%vol. \\
\hline MW & Molecular weight, in kg/kmol. \\
\hline$R D$ & Reactor demand $\left(\mathrm{RD}_{\mathrm{H} 2}\right)$ or generation $\left(\mathrm{RD}_{\mathrm{LIG}}\right)$, in $\mathrm{Nm}^{3} / \mathrm{h}$. \\
\hline G & Mixed gas and liquid stream, in $\mathrm{kmol} / \mathrm{h}$. \\
\hline$H C$ & Liquid hydrocarbons flow, in $\mathrm{m}^{3} / \mathrm{h}$. \\
\hline$r d$ & Specific demand $\left(\mathrm{rd}_{\mathrm{H} 2}\right)$ or generation $\left(\mathrm{rd}_{\mathrm{LI}}\right)$, in $\mathrm{Nm}^{3} \mathrm{H}_{2} \cdot\left(\mathrm{m}^{3} \mathrm{HC}\right)^{-1}$. \\
\hline $\mathrm{ksOl}_{\text {se, gasHC }}$ & Total gas solubility in hydrocarbons at separator se, in $\mathrm{Nm}^{3} / \mathrm{m}^{3} \mathrm{HC}$. \\
\hline$k_{s o l}{ }_{s e, H 2 L I G}$ & Relative $\mathrm{H}_{2}$ / LIG gas/liquid solubility coefficient. Non-dimensional fraction. \\
\hline $\mathrm{ksol}_{\text {se,MWLIG }}$ & MW $W_{\text {LIG }}$ gas/liquid solubility coefficient. \\
\hline$Y$ & Total molar fraction of a gas and liquid stream, in \%. \\
\hline$\rho$ & Density of a liquid stream, in $\mathrm{kg} / \mathrm{m}^{3}$. \\
\hline$P$ & Manometric pressure, in kg/cm² or bar $^{*}(1 \mathrm{bar} \leftrightarrow 100 \mathrm{kPa})$. \\
\hline
\end{tabular}

$\forall s e \in$ Separators

Table 4.1 - Descriptions of variables and subscripts. ${ }^{*}$ For simplicity these pressure units are considered equivalent, actual conversion is: $1 \mathrm{~kg} / \mathrm{cm}^{2} \leftrightarrow 0.981$ bar 


\begin{tabular}{|l|l|}
\hline$T$ & Temperature, in $\mathrm{OC}$. \\
\hline$k$ & A node within the network. \\
\hline \multicolumn{2}{|l|}{ Subscripts } \\
\hline in & A reactor within the network. \\
\hline out & The variable represents an inlet. \\
\hline$o$ & The variable represents an outlet. \\
\hline$p g$ & The purified gas of a permeation membrane. \\
\hline$z$ & The gas purge of a permeation membrane. \\
\hline se & A purification membrane within the network. \\
\hline$d$ & A separator within the network. \\
\hline mea & Orifice plate design value. \\
\hline rec & Measured value. \\
\hline
\end{tabular}

Table 4.2 - Engineering units used, unless explicitly stated otherwise.

\begin{tabular}{|l|l|l|l|l|l|l|l|}
\hline $\begin{array}{l}\text { Liquid } \\
\text { flow }\end{array}$ & $\begin{array}{l}\text { Gas } \\
\text { flow }\end{array}$ & $\begin{array}{l}\text { Gas } \\
\text { purity }\end{array}$ & $\begin{array}{l}\text { Molecular } \\
\text { weight }\end{array}$ & Pressure & Temperature & Time & $\begin{array}{l}\text { Cost } \\
\text { Profit }\end{array}$ \\
\hline $\mathrm{m}^{3}$ & $\mathrm{Nm}^{3} / \mathrm{h}$ & $\% \mathrm{vol}$ & $\mathrm{kg} / \mathrm{kmol}$ & $\mathrm{kg} / \mathrm{cm}^{2} \mathrm{~g}$ & ${ }^{\circ} \mathrm{C}$ & $\mathrm{h}$ & Euros $/ \mathrm{h}$ \\
\hline
\end{tabular}

It must be noticed that, further details such as operation constraints (e.g.: compressors' capacities and constraints on pipelines), and actual process units' flow diagrams are confidential, thereby not available for disclosure. However, those are incorporated into the model and its constraints appropriately.

Taking into account the much faster dynamics of gaseous flowrates compared to the dynamics of chemical hydrogen demand in reactors, the stationary assumption of hydrogen distribution is bearable. Thereby, the variables of the model are: flows $(F)$, purities $\left(X_{H 2}\right.$ and $\left.X_{L I G}\right)$, molecular weights of hydrogen and light ends $\left(M W_{H 2}\right.$ and $M W_{L I G}$, respectively) of all streams and hydrogen consumption in the reactors. 
For the sake of clarity, the deterministic model of the process network refers to equations 4.1.a-d, 4.2.a-e, 4.3.a-e, 4.4.a-f (or 4.1-4 for short), and other equations. Altogether represent the full plant mathematical model used in this work.

The data reconciliation (DR) problem requires a certain degree of redundancy in the measurements and is formulated as the following nonlinear programming (NLP) problem:

$$
\min _{F_{i}, X_{i}, M W_{i}, \varepsilon_{i}} \sum_{j \in M} \alpha_{j} c^{2}\left[\frac{\left|e_{j}\right|}{c}-\log \left(1+\frac{\left|e_{j}\right|}{c}\right)\right]+\sum_{i \in S} \alpha_{i} \varepsilon_{i}^{2}+\sum_{k \in M} \alpha_{k} \operatorname{Reg}_{k}
$$

Subject to:

process network model (4.1-4)

operational and range constraints

$$
\begin{aligned}
& F_{i, \text { min }}-\varepsilon_{F i} \leq F_{i} \leq F_{i, \text { max }}+\varepsilon_{F i} \quad \varepsilon_{F i} \geq 0 \\
& X_{i, \text { min }}-\varepsilon_{X i} \leq X_{i} \leq X_{i, \text { max }}+\varepsilon_{X i} \quad \varepsilon_{X i} \geq 0 \\
& M W_{L I G, i, \min }-\varepsilon_{M W i} \leq M W_{L I G, i} \leq M W_{L I G, i, \max }+\varepsilon_{M W i} \quad \varepsilon_{M W i} \geq 0
\end{aligned}
$$

Where:

$$
\begin{gathered}
e_{j}=\eta_{j}\left(F_{r e c, j}-\beta_{j} \cdot F_{j, \text { mea }}\right) \quad j \in M \\
e_{m}=\eta_{m}\left(X_{r e c, m}-X_{m, m e a}\right) \quad m \in M \\
\beta_{j}=\sqrt{\frac{T_{d}+273}{\left(P_{d}+1\right) \cdot M W_{j}}} \sqrt{\frac{\left(P_{j}+1\right) \cdot M W_{d}}{T_{j}+273}}
\end{gathered}
$$


The above NLP minimizes the function (4.5.a) of the errors $e_{j}$ between the measured flows $F_{j, \text { mea }}$ and purities $X_{m \text {, mea }}$, and the same magnitudes computed with the model under the links imposed by the model (4.1-4) and other operational and range constraints. The coefficients $\beta$ represent the compensation factors, and the variables $\varepsilon$ are slack variables to ensure feasibility in the range constraints, while $R e g$ are regularization terms to avoid sharp changes. Index $i$ expands to all streams $(S)$ across the network model, while indices $j$ and $m$ refer to plant measurements within set $M$. Notice that instead of the common sum of squares of the errors, a robust M-estimator (a.k.a.: maximum-likelihood type estimators) as the Fair function has been used, which is similar in shape to the sum of squared errors for small values of the error but grows slower for larger ones limiting the effect of gross errors in the data (Arora and Biegler, 2001; Huber, 2011; Nicholson et al., 2014; Özyurt and Pike, 2004).

The data reconciliation problem is a large-scale NLP that is formulated and solved with a simultaneous approach in the General Algebraic Modeling System (GAMS ${ }^{\circledR}$, GAMS Development Corporation, 2019) using IPOPT (Wächter and Biegler, 2006) as the optimization algorithm. The implementation involves more than 4400 variables and 4700 equality and inequality constraints. It takes less than five Central Processing Unit (CPU) minutes in a PC with i7 processor and 8 GB RAM, giving robust results against gross errors and helping to detect faulty instruments.

\subsubsection{NETWORK RTO}

Once a sensible model and reliable corrected measurements are available, one can formulate the network optimization problem (4.6) as finding the production and redistribution of $\mathrm{H}_{2}$ in the network and the value of the hydrocarbon loads to the consumer plants that maximizes the value associated to the loads taking into account the cost of generating hydrogen, which corresponds to the cost function: 


$$
\max _{H C_{1, \ldots, 4}, F_{H 2,1, \ldots, 2}, R_{1, \ldots, 4}} \sum_{i=1}^{4}\left(p_{H C i} H C_{i}-p_{R i} R_{i}\right)-\sum_{j=1}^{2} p_{H 2} F_{H 2, j}
$$

Where $\mathrm{p}$ represent prices $H C$ are hydrocarbon loads, $F$ fresh hydrogen and $R$ deals with the compression cost of the recycled one.

This function is maximized respecting all constraints and without changing the way the reactors are operated, that is:

- Maintaining the current specific consumption of $\mathrm{H}_{2}\left(r d_{H 2}\right)$, LIG generation $\left(r d_{L I G}\right)$ and their properties (purity and molecular weight) at each reactor,

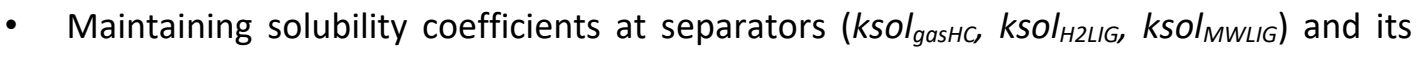
properties (purity and molecular weight).

These values are estimated every two hours from the data reconciliation step and are expected to be the same in the (near) future, if there is no change in hydrocarbon feed quality.

In the optimization, besides the network model, the main constraints refer to the process operation (e.g.: ranges, $\mathrm{H}_{2} / \mathrm{HC}$, compressors capacity and maximum purity) and refinery planning specifications. Main decision variables include production of fresh hydrogen, feeds to consumer plants, hydrogen flows and recirculation, purges, purities and membranes operation.

The RTO is solved as an NLP problem in the GAMS ${ }^{\circledR}$ system. It involves nearly 2000 variables and more than 1800 equality and inequality constraints and is solved with a simultaneous approach and the IPOPT algorithm in less than one minute CPU time.

\subsubsection{SYSTEM ARCHITECTURE FOR OPTIMIZATION}

The data reconciliation and hydrogen network management RTO are implemented according to the architecture displayed in Fig. 4.5. 
Data and measurements from the hydrogen network are stored regularly in the real-time information system of the refinery (PI system). Values of each of them are read every two hours from the PI system to be processed in the DR-RTO application which resides in a dedicated PC. The application is composed of several modules as shown in the left hand side of Fig. 4.5. The data acquisition module reads 171 flows and 18 purity measurements, plus other variables and configuration parameters from the PI system (temperatures, pressures, valve openings, etc.) totalizing around 1000 variables, averaging them in two-hour periods to smooth the effects of transients and disturbances. Data treatment is a critical component that contains a set of rules dedicated to detect faults and information inconsistences in the raw data and decides which options, variable ranges, etc. are the most adequate ones in the mathematical formulation of the problems. In addition, this module detects when a plant is out of service or a hydrogen header has modified its connectivity, such that its associated equations should be removed or changed in the network models. To implement this variable structure operation, the models are formulated as a superstructure that includes binary variables such that, according to the analysis of the data treatment module, the model can be adapted automatically to the state and configuration of the plants and headers.

Then, the treated data and constraints are sent to the data reconciliation module that solves the corresponding optimization problem and provides updated and reliable information and parameters to the network optimization module (named as Optimal Redistribution in Fig. 4.5). Finally, the information from the data reconciliation (DR) and the network optimization are used to compute some Resource Efficiency Indicators (REIs), and all of them are sent back to the PI system, where they are available to all potential users. Further details of REls implementation and their usefulness for decision support in refinery hydrogen network are presented by Galan et al. (2017). A detailed description of REIs and their role in real-time monitoring and optimization in the process industry is covered by Krämer and Engell (2018), while a comprehensive guide for REls development is presented by Kujanpää et al. (2017). 


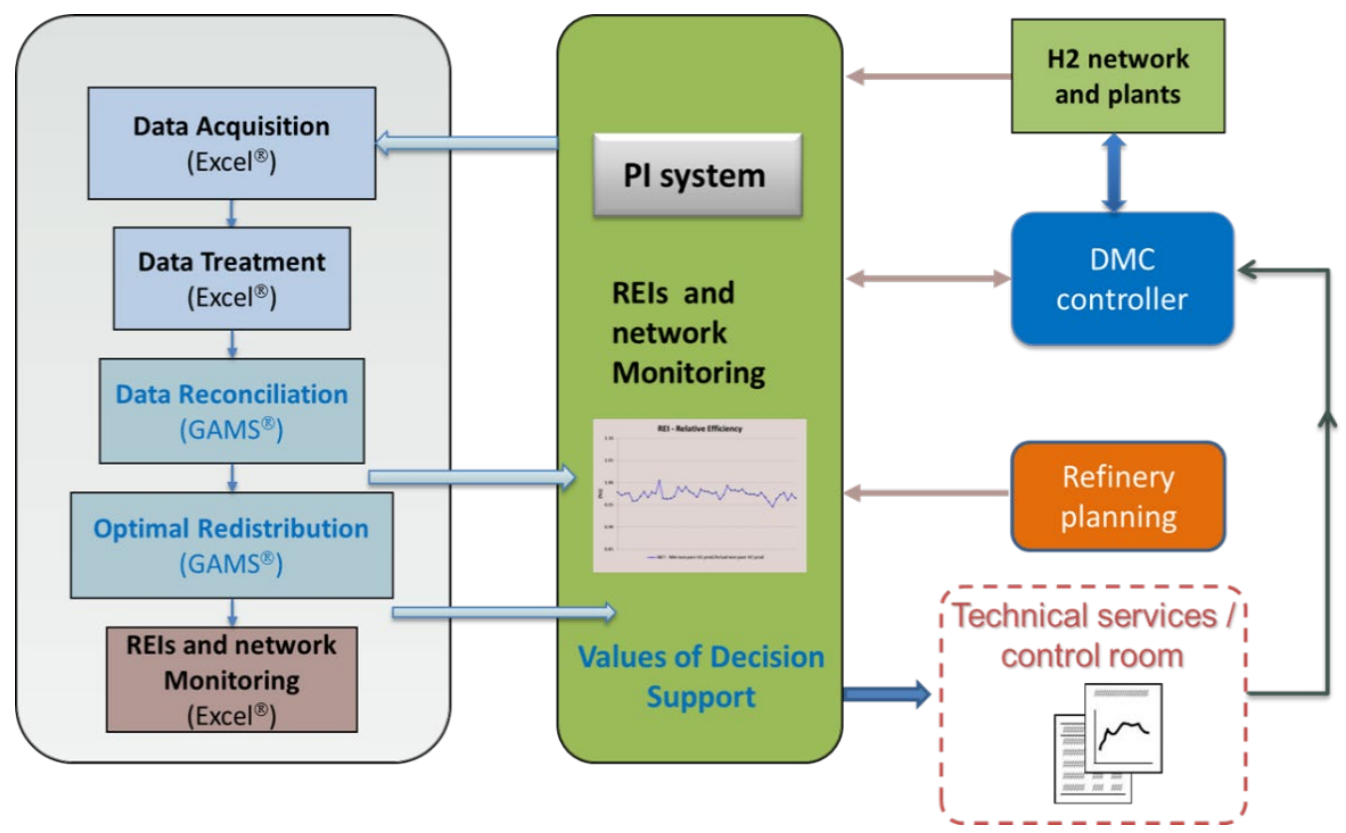

Fig. 4.5 - Schematic of the system's architecture displaying the DR-RTO module on the left hand side, the PI system in the center and process and other control and planning elements on the right hand side.

A first benefit of the system is providing improved process information and, in particular:

- $\quad$ an indication of possible faulty instruments

- reliable balances of hydrogen

- $\quad$ values for unmeasured quantities (purities, molecular weights, hydrogen consumption and others) not available previously

- data for computing REls that allow better monitoring of the operation of the network

Regarding the implementation of the solutions of the optimizer, ideally, the optimal values calculated should be sent as set-points to the network control system, either directly to the flow controllers or following the traditional architecture as in Fig. 4.1. Nevertheless, the static nature of the RTO and the low frequency of its execution brings several problems as the implementation of the optimal values has to be applied to the process taking into account the time evolution of variables. In particular, HC loads and 
hydrogen production have to be changed dynamically at a higher frequency to balance hydrogen production and consumption. In the same way, due to the presence of disturbances, changing aims, etc., constraints' fulfilment requires dynamic actions to be performed at a higher rate, and changes in hydrogen flows may interact among them so that a proper implementation of the RTO solution would require multivariable control to take care of the interactions. Because of that, a different approach has been considered.

\subsubsection{IMPLEMENTING NETWORK OPTIMIZATION IN REAL-TIME}

A direct way of incorporating dynamics into the system, solving simultaneously the problem of possible inconsistencies between the non-linear RTO model and the linear one typically used in the MPC layer, is to formulate a single integrated dynamic optimization problem as mentioned in the introduction. Nevertheless, it is not realistic maintaining and operating in real-time a dynamic data reconciliation and dynamic RTO system involving 18 plants due to its large scale.

A different alternative, somewhere in the middle between sending set-points from a RTO to a MPC and direct dynamic optimization with economic aim, was considered and implemented in the refinery. For implementation, it takes advantage of the fact that some commercial MPCs, e.g. DMC+, are actually composed of two layers: a Dynamic Matrix Controller (DMC) to compute control actions, and a local optimizer on top that, using Linear Programming (LP) and sharing the same linear dynamic models as the DMC, computes on-line targets for the multivariable controller minimizing a user defined economic function.

The methodology is represented in Fig. 4.6, and basically consists of analyzing the network RTO solutions and extract from them optimal policies that are consistently recommended by the optimizer. This means, understanding the logic behind the solutions and identifying variables that should be maximized or minimized to achieve an overall network optimal management. Nonetheless, variable specific set-points depend on the process constraints or planning specifications. Therefore, the decision-making process, rather than being automatically translated downstream in the control pyramid, provides 
operation directions to the optimization layer of the DMC. These policies, or directions, represent targets (variables), which are maximized or minimized in the LP layer of the DMC considering controlled and manipulated variables' specific weights. These weights reflect the priorities and costs of the steady state process. The LP determines the optimal values compatible with the actual process model, process state and constraints and generates the corresponding set points to the DMC controller, which finally, taking into account systems dynamics and interactions, will compute current and future hydrogen and hydrocarbon set points to be given to the individual low level flow controllers of the DCS of the control room. A comprehensive description and discussion of the integration of the RTO and DMC in this process network is addressed by de Prada et al. (2017).

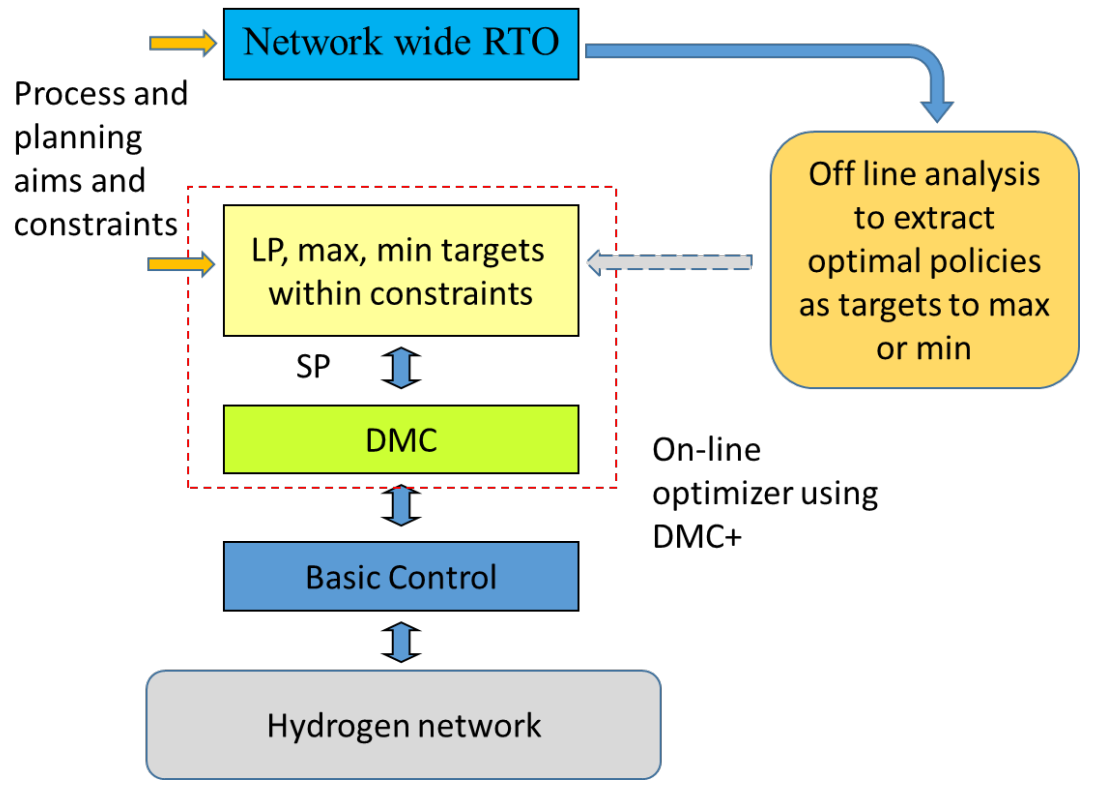

Fig. 4.6 - Schematic representing the methodology for on-line implementation of RTO policies.

In the case considered, the optimal policies identified were:

- Losses from the HPS of a plant to fuel gas, required to avoid LIG accumulation, should be made at the lowest hydrogen purity compatible with the one required at the reactor input and the $\mathrm{H}_{2} / \mathrm{HC}$ minimum ratio, but the LPH purity should be maximized 
to increase hydrogen re-use. It must be noticed that, the higher the purity in the LPH, the more utilization of that gas for the process units.

- The hydrogen unbalance in the network, that is, hydrogen generated minus hydrogen consumed in the reactors, reflects in the LPH pressure, so losses to fuel gas from this header should be minimized with a minimum to guarantee unsaturated operation of the pressure controller. This is a typical operational constraint, basically linked to the pressure valve controller.

- Maximization of the hydrocarbon load to the consumer plants, which is the most important target, and can be made until either maximum hydrogen capacity is reached or another technical constraint is active.

- $\quad$ Sending higher purity hydrogen (H4) to LPH should be minimized as purity degrades.

The system was implemented at the refinery shown in Fig. 4.5, but with the DMC controlling only the six most important plants from the hydrogen use point of view as a compromise between maintenance and development costs and potential benefits as in Fig. 4.7. A detailed description of the validation process of this deployment is addressed by Galan et al. (2018), while de Prada et al. (2017) presents an analysis of the integration of the RTO and MPC applications in this case study.

The DMC controller manages two hydrogen producers $(\mathrm{H} 3, \mathrm{H} 4)$ and four consumer plants $(\mathrm{G} 1, \mathrm{G} 3, \mathrm{G} 4, \mathrm{HD} 3)$ and was developed and implemented by the refinery team. It is based on linear models obtained by identification using data from step-tests that forms a dynamic matrix involving 12 manipulated variables and 29 controlled ones. The main manipulated variables refer to the set points of hydrocarbon loads to the consumer units, fresh hydrogen production, hydrogen feed to the consumers from the high purity header, and supply of hydrogen from one of the platformer plants. The main controlled variables are hydrogen partial pressure in the reactors of the consumer plants, losses to fuel gas from the LPH (valve opening), recycle purity and HP losses to FG from some plants, hydrocarbon loads and valve openings to avoid control saturation. 


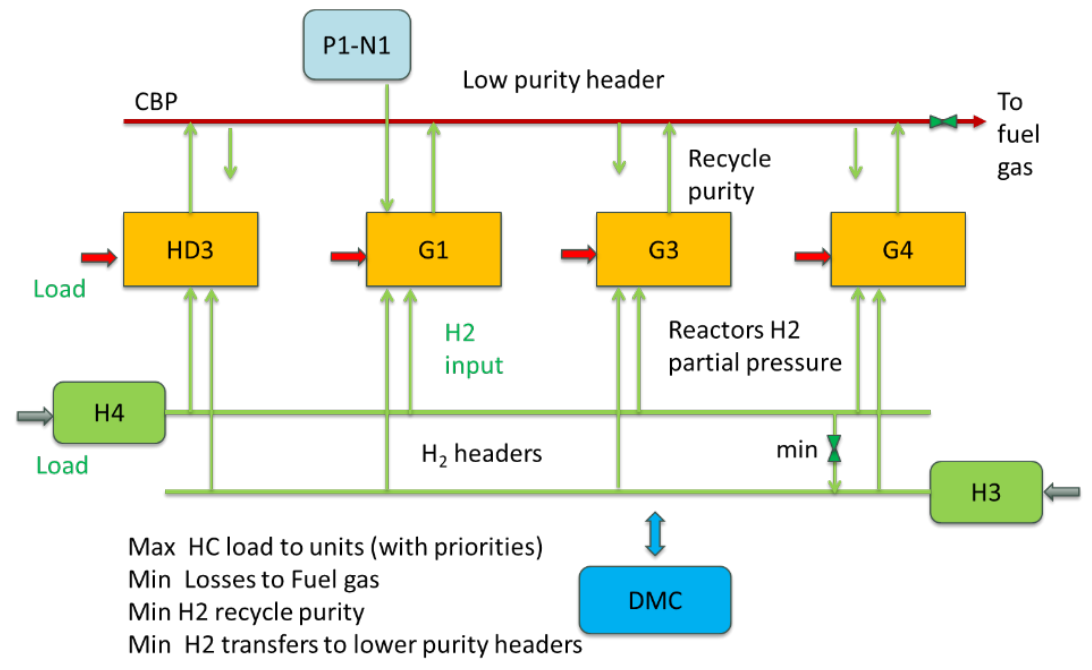

Fig. 4.7 - Diagram of the DMC controlling the operation of two hydrogen producers $\mathrm{H} 3$ and $\mathrm{H} 4$ and four consumers G1, G3, G4 and HD3, with the main controlled hydrogen flows and HC loads.

The cost function at the LP layer combines four targets that together synthesize the solution of the RTO:

- Maximize hydrocarbon loads to the consumer plants

- Minimize losses from the LPH to FG

- Minimize hydrogen purity in the recycles of the consumer plants

- Minimize hydrogen transfers from higher to lower purity headers

The corresponding variables are linked to the manipulated variables through the linear process model, so that the optimization problem is linear and can be solved in a short time. The LP / DMC runs with a sampling time of one minute, giving consistent results for many months. In parallel, the network RTO is executed every two hours being operated as a DSS for the whole network and allowing the supervision of the DMC application. As an example of results, Fig. 4.8 (bottom) presents the total optimal and actual hydrocarbon load to the HDS plants for a period of ten days, showing good performance. However, in the same time window it is seen still a gap in the $\mathrm{H}_{2}$ sent to FG when comparing the reconciled value to the optimal (Fig. 4.8, top). This gap is mainly explained by the fact that, the purification membranes across the network are operated 
manually and are out of the scope of the DMC, though considered in the RTO. These results showcase the importance of the optimal operation of purification membranes at network level (ideally automated), and their impact in the economy of the process. A thorough discussion of this finding is addressed by Galan et al. (2018).
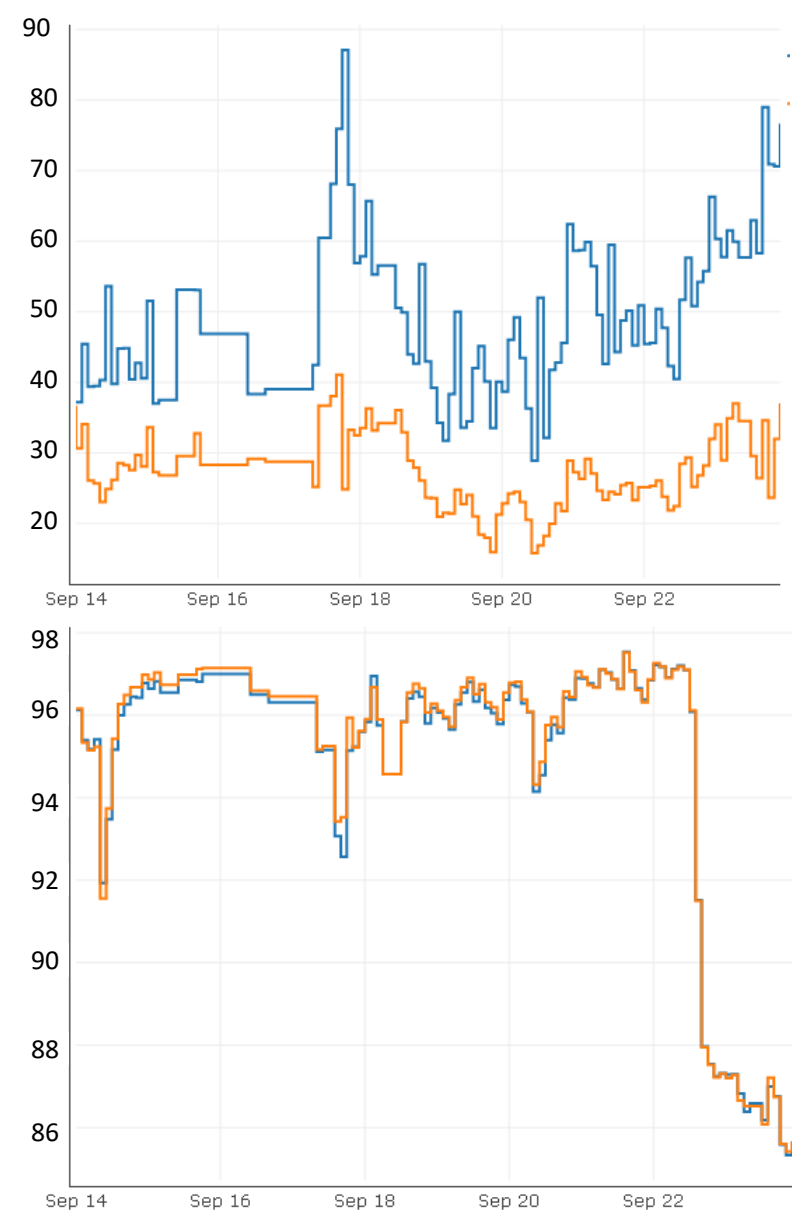

Fig. 4.8. Evolution of optimum (orange) and reconciled (blue) plant-wide figures over ten days. Top: hydrogen sent to FG header (\%reference). Bottom: total hydrocarbons processed (\%reference). 


\subsection{INCORPORATION OF EXPLICIT UNCERTAINTY}

In Fig. 4.8 (top and bottom), is seen that the optimal conditions change significantly over time. In fact, the refinery is subjected to potentially large changes every two to three days when it receives new crude oil from ships, not to mention new production targets imposed by market demands. Consequently, hydrogen demand at reactors changes as well, impacting on hydrogen demand across the network.

In this section, uncertainty in the hydrogen demand at reactors is considered in the decision-making process explicitly. For this purpose, network dynamics considerations are proposed such that, slower plants' (low frequency dynamics) variables are to be decided ahead of time (here-and-now decisions), compared to faster plant's (high frequency dynamics) variables. For instance, set-point changes in production units (i.e.: $\mathrm{H} 3$ and $\mathrm{H} 4$ ) typically take around two hours to realize and actually impact on the consumer unit. Thereby, actual gas demand at reactors should have been met and produced at hydrogen units two hours earlier than actual consumption. Otherwise, an excess or defect in hydrogen demand, along with its economic consequences, is faced. Based on this sequence, it is possible to formulate a two-stage stochastic framework for the hydrogen network management problem with uncertain parameters. This approach is introduced and analyzed by Gutierrez et al. (2018) in a previous work over this case study.

Changes in the crude oil reflect in changes in the hydrogen consumption of the reactors of the HDS plants that are difficult to predict, creating transients where the performance of the network may suffer degradation. One may wonder if incorporating this uncertainty explicitly in the decision making process would improve significantly the results obtained.

At the RTO level, this is done updating the model and network information at regular intervals by means of data reconciliation. Nevertheless, it is well known that, even with data reconciliation, if the model has structural errors the optimum computed with the model may not correspond to the real process optimum. Alternatively, we can 
consider different possible values of the uncertain variables and optimize considering the worst case, following a robust optimization approach (Ben-Tal and Nemirovski, 2002). This option chooses the values of the decision variables that guarantee fulfilment of all constraints in all scenarios, but provides very conservative solutions as they are fitted to the worse case. Another alternative approach may be multi-stage stochastic optimization, which takes into account that some decisions that influence the future behavior of the process have to be made at current time (here-and-now) without knowing the value of the uncertain parameters (e.g.: $\mathrm{H}_{2}$ demand at reactors) but, in the future, new information can be available that reveals the value of the uncertainty, so that particular correction actions (recourse) can be made in the future according to the specific scenario that may take place. In this respect, two-stage stochastic programming is the simplest formulation possible, where first stage variables represent here-and-now decisions and second stage variables represent recourse decisions, which are scenario dependent.

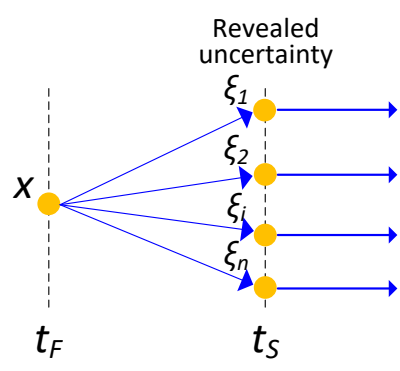

(a)

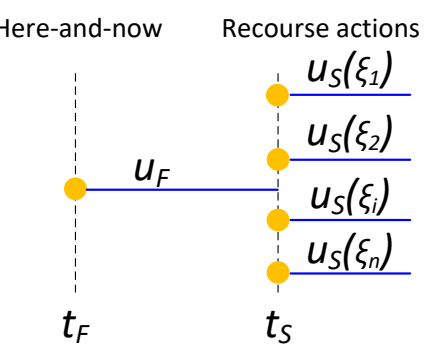

(b)

Fig. 4.9. Schematic of the main concepts behind two-stage stochastic optimization and scenario tree representation.

The concept is illustrated in Fig. 4.9, where a scenario tree is represented for a twostage stochastic model. On the left hand side (a) the system has a state $x$ at time $t_{F}$ and a decision $u_{F}$ (known as first-stage variables) has to be made considering all possible values $\xi_{i}$ of the uncertainty, a scenario is defined as the $\xi_{i}$ realization from instant $t_{F}$ to $t_{S}$ through nodes. After applying $u_{F}$, the system will evolve in $t_{S}$ to different states depending on the specific value of $\xi_{i}$, but if this value were known at $t_{F}$, we could compute a specific optimal 
decision $u_{s}\left(\xi_{i}\right)$ for each value of $\xi_{i}$ in the period of time starting at $t_{s}$ for the remaining variables (recourse variables), as in Fig. 4.9 b. The following sections study the value of the stochastic approach, by means of formulating a two-stage stochastic programming (TSSP) problem applied to the hydrogen network in order to evaluate the convenience of its implementation in the RTO framework.

\subsection{DESCRIPTION OF THE TSSP PROBLEM}

Main elements in the formulation of the optimal management of the hydrogen network as a two-stage stochastic optimization problem are: the identification of the uncertainty source, the scenarios definition with their likelihood of realization, and selection of meaningful first and second stage variables. Regarding the objective function, the simplest approach is to formulate the deterministic equivalent problem (DEP) of the minimization as in (4.7.a-f). A detailed discussion on alternative formulations of TSSP problem could be found in Birge and Louveaux (2011).

$$
\min _{u_{F}, u_{S\left(\xi_{i}\right)}} J_{F}\left(u_{F}\right)+\mathbb{E}\left\{J_{S}\left(u_{F}, u_{S}\left(\xi_{i}\right), x_{S}\left(\xi_{i}\right)\right)\right\}
$$

Subject to:

$$
\begin{aligned}
& \mathbb{E}\left\{J_{S}\left(u_{F}, u_{S}\left(\xi_{i}\right), x_{S}\left(\xi_{i}\right)\right)\right\}=\sum_{i=1}^{n} J_{S}\left(u_{F}, u_{S}\left(\xi_{i}\right), x_{S}\left(\xi_{i}\right)\right) \cdot \pi\left(\xi_{i}\right) \\
& h_{F}\left(x_{F}, u_{F}\right)=0 \\
& g_{F}\left(x_{F}, u_{F}\right) \leq 0
\end{aligned}
$$




$$
\begin{aligned}
& h_{S}\left(x_{F}, u_{F}, u_{S}\left(\xi_{i}\right), x_{S}\left(\xi_{i}\right)\right)=0 \quad \forall \xi_{i} \in \Xi, \\
& g_{S}\left(x_{F}, u_{F}, x_{S}\left(\xi_{i}\right), u_{S}\left(\xi_{i}\right)\right) \leq 0 \quad \forall \xi_{i} \in \Xi
\end{aligned}
$$

Where: $(\cdot)_{F}$ refers to variables or functions in the first stage and $(\cdot)_{S}$ denotes the ones in the second stage, while the decision variables are denoted as $u$ and the remaining variables as $\mathrm{x}$. The uncertainty is represented by the parameters $\xi_{\mathrm{i}}$ that can take values within a set $\Xi$ according to a certain discrete probability distribution, for which the probability of occurrence $\left(\pi\left(\xi_{i}\right)\right)$ is known (4.7.b). This set is discrete and finite with $n$ elements, (i.e.: $\xi_{i}, i=1,2,3, \ldots, n$ of elements is considered). These $n$ elements constitute the scenarios that will represent the uncertainty realizations. In the objective function the sum over all scenarios i represents the expected value of the objective function over the second stage variables (4.7.b).

The cost function is composed of two terms: The first one, $J_{F}$, is the cost in the first stage which depends on the first stage decisions $u_{F}$. These are decisions that are taken and applied at current time without knowing the particular realization of the uncertainty $\xi$ and will be maintained over the time horizon covered by the optimization problem.

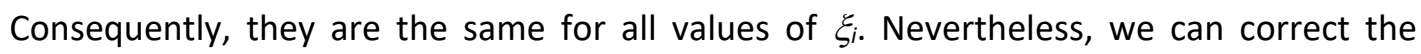
effects of the $u_{F}$ decisions once the value of the $\xi_{i}$ parameters are revealed, using the recourse variables $u_{S}(\xi)$ that take a particular value for each realization of the uncertainty $\left(\xi_{i}\right)$. The second term of the cost the weighted summation over all the scenarios with corresponding probabilities $\pi_{i}$, represents the effect of these second stage corrections on the total value of the cost function, which also depend on the $u_{F}$ decisions.

The variables of the problem have to satisfy the constraints imposed by the model $h($.$) and additional inequality constraints g($.$) in every stage for all possible scenarios$ considered (n). In (4.7), the corresponding equations, that depend on the stochastic parameter $\xi$, should be interpreted as being satisfied with probability one. 


\subsubsection{UNCERTAINTY SOURCE}

Hydrogen gas in a refinery is basically a utility, for it is demanded and consumed in process units and it should be enough to satisfy the process requirements at all times. The deterministic problem tackles the optimal hydrogen management problem assuming that hydrogen demand of each plant is to be calculated exactly using the results of the DR problem. However, this concept does not hold when the refinery is facing crude oil changes, which typically imply hydrogen demand swings as well. In these situations, predictions of hydrogen demand at the plant level are usually inaccurate due to the fact that hydrocarbon cuts properties may be estimated with large errors, which make them the main source of uncertainty. Figure 4.10 presents a simplified oil refinery schematic representing the different intermediate cuts fed to hydrogen consumer units (i.e.: HDS, HDT, HDC), which will be impacted by changes in the hydrocarbon properties and ultimately lead to hydrogen demand changes. Therefore, a scenario tree representation is applicable in this context as seen in Fig. 4.9. In addition, in most cases hydrogen demand affects all consumers in the same direction (i.e.: increase or decrease) as a consequence being fed by a unique crude oil source (see Fig. 4.10). It must be present that refinery hydrogen networks are very specific due to all the features described before. Other gas networks case studies available in literature, such as the one by Li et al. (2017) for natural gas networks, may differ in most of the assumptions and features, though the stochastic approach still holds in all. 


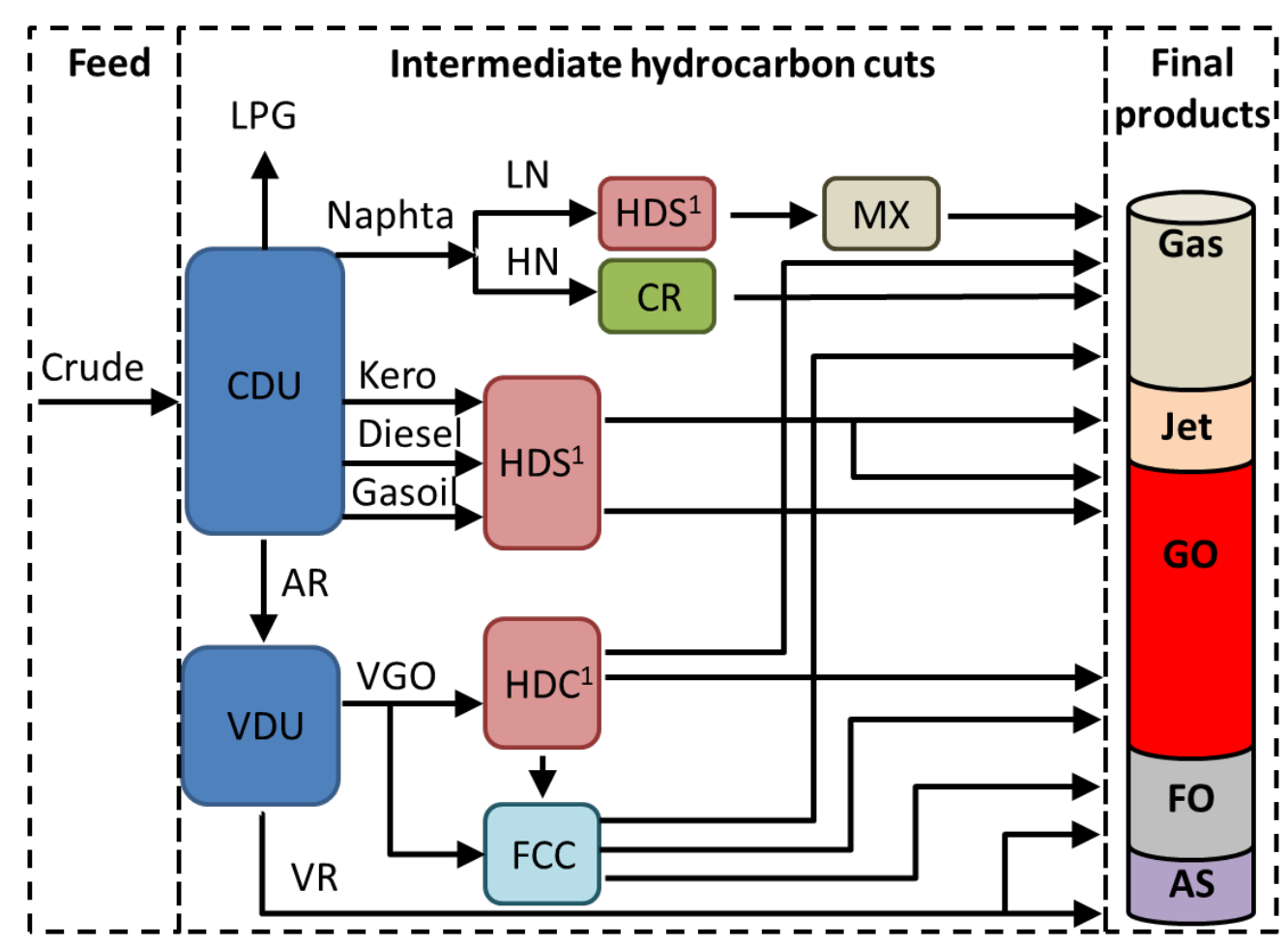

Fig. 4.10. Simplified schematic of an oil refinery, identifying the main intermediate cuts fed to process units. CDU - Crude distillation unit. VDU - Vacuum distillation unit. HDS Hydrodesulfurization unit. HDC - Heavy oil desulfurization unit. FCC - Fluidized catalytic cracking. CR - Catalytic reforming. MX - Merox sweetening. LPG - Liquefied petroleum gas. Kero - Kerosene. LN / HN - Light and heavy naphta, respectively. AR - Atmospheric residue. VR - Vacuum residue. Gas - Gasoline. Jet - Aviation jet fuel. GO - Commercial gasoil. FO - Fuel oil. AS - Asphalt. ${ }^{1}$ Major hydrogen consumer.

\subsubsection{SCENARIOS DEFINITION}

Given different potential hydrogen demands at plant level is possible to link those to a probability of occurrence $\left(\pi\left(\xi_{i}\right)\right)$, which will be revealed only after the first stage decisions are due. Therefore, each scenario is identified with a likelihood of realization of a hydrogen demand at plant level. It should be borne in mind that this idea narrows down the search for first and second stage variables, since the former remain equal at all scenarios, which is generally represented by the so called non-anticipativity constraints (NACs). 


\subsubsection{FIRST AND SECOND STAGE VARIABLES}

As a consequence of the network dynamics, explained in section 4.2, hydrogen production decisions at generation units (i.e.: $\mathrm{H} 3$ and $\mathrm{H} 4$ ) precede actual plant demand at consumer units by around two hours. In other words, hydrogen demand at any given time should be met by hydrogen production decisions on hydrogen rates of the past two hours. However, consumer plants have much faster dynamics and corrections to cope with most of the changes in feed quality can be made within minutes. Due to the fact that the uncertainty source is from feed quality, which in turn reflects into hydrogen demand at the plant level, scenarios affect all consumer plant variables and headers. Additionally, hydrogen production has to be set two hours before it is actually demanded. Therefore, in the TSSP formulation the first stage variables are all related to the hydrogen production units, $\mathrm{H3}$ and $\mathrm{H} 4$. The rest of the network variables are all subjected to scenarios hence defined as recourse or second stage variables.

\subsection{RISK NEUTRAL PROBLEM STATEMENT}

Given the hydrogen network of an oil refinery, with production and consumption of hydrogen, and hydrocarbons processed in consumer plants. The problem is to determine the hydrogen production rate at time $t_{0}$ of each producer, such that plants demands' are satisfied for all possible scenarios, complying with operational restrictions. The objective is to maximize the expected profit of the network operation (4.8), considering hydrogen production costs and revenues from hydrocarbon processing at all scenarios.

$$
\begin{aligned}
\max _{F_{H_{2}}, H C_{i}\left(\xi_{i}\right), R_{i}\left(\xi_{i}\right)} J_{F}\left(-\sum_{i=1}^{2} p_{H_{2} i} \cdot F_{H_{2} i}\right) \\
+\mathbb{E}\left\{J_{S}\left(\sum_{j=1}^{4} p_{H C_{i}} \cdot H C_{i}\left(\xi_{j}\right)-p_{R_{i}} \cdot R_{i}\left(\xi_{j}\right)\right)\right\}
\end{aligned}
$$


Here the process model and constraints are the same as in the deterministic case (i.e.: $h(\cdot)$ and $g(\cdot))$, but evaluated for every scenario $(4.7 . b-f)$, which largely increases the number of variables and equations. The first stage cost $\left(J_{F}\right)$ corresponds to the production cost of fresh hydrogen, while the second stage $\left(J_{S}\right)$ includes the expected value of the hydrocarbons processed and the cost of the hydrogen recycles. The aim is to maximize the hydrocarbon load $(H C)$ to consumer plants, minimize the use of fresh hydrogen generated in the steam reforming plants $\left(F_{H}\right)$ and minimize the internal recycles of hydrogen $(R)$ in the consumer plants, considering all possible values of the uncertainty. $u_{s}$ refers to the remaining variables of the model.

This TSSP formulation is known as deterministic equivalent problem (DEP) since it is solved as a single monolithic optimization problem over all the scenarios.

\subsection{EVALUATION OF THE VALUE OF THE STOCHASTIC SOLUTION}

\subsubsection{SCENARIOS ASSESSED}

In particular, a formulation with nine scenarios is presented as case study in this section. Table 4.3 displays details of scenarios conditions, which represent feasible transitions towards a higher hydrogen demand resulting from higher sulfur content crude oil. In fact, the key representation of these scenarios in the model is by multiplying 4.2.d and 4.2.e by their corresponding change coefficient at each scenario (4.9.a-b). The rest of the model equations remain unchanged, except for the addition of the scenario dimension to each second stage variable. It is assumed that other realizations are negligible. Therefore, these nine scenarios represent all meaningful $\xi_{i}$, such that the probability of occurrence $(\pi)$ of the sum of all equals one (10). All values are presented in per one unit (e.g.: 1.1 implies ten percent increase).

$$
R D_{H 2, r, \xi}=H C_{i n, r, \xi} \cdot\left(r d_{H 2, r} \cdot H_{2 D E M, \xi}\right)_{r, \xi}
$$




$$
\begin{aligned}
& R D_{L I G, r, \xi}=H C_{i n, r, \xi} \cdot\left(r d_{L I G, r} \cdot L I G_{G E N, \xi}\right)_{r, \xi} \\
& \forall \xi \in \Xi \\
& \sum^{9} \pi\left(\xi_{i}\right)=1
\end{aligned}
$$

Table 4.3 - Scenario specific hydrogen demand $\left(\mathrm{H}_{2 \mathrm{DEM}}\left(\mathrm{S}_{\mathrm{i}}\right)\right)$, light ends generation $\left(\operatorname{LIG}_{\mathrm{GEN}}\left(\mathrm{S}_{\mathrm{i}}\right)\right)$ and probability of occurrence $\left(\pi\left(\mathrm{S}_{\mathrm{i}}\right)\right)$, for each scenario $\left(\mathrm{S}_{\mathrm{i}}\right)$. $\mathrm{H}_{2 \mathrm{DEM}}\left(\mathrm{S}_{\mathrm{i}}\right), \mathrm{LIG}_{\mathrm{GEN}}\left(\mathrm{S}_{\mathrm{i}}\right)$ and $\pi\left(\mathrm{S}_{\mathrm{i}}\right)$ values are presented in per one fractions.

\begin{tabular}{|l|l|l|l|l|l|l|l|l|l|}
\hline & S1 & S2 & S3 & S4 & S5 & S6 & S7 & S8 & S9 \\
\hline $\mathrm{H}_{2 \mathrm{DEM}}$ & 1 & 1.1 & 1.2 & 1 & 1.1 & 1.2 & 1 & 1.1 & 1.2 \\
\hline LIG $_{\text {GEN }}$ & 1 & 1 & 1 & 1.1 & 1.1 & 1.1 & 1.2 & 1.2 & 1.2 \\
\hline$\pi$ & 0.36 & 0.15 & 0.09 & 0.15 & 0.0625 & 0.0375 & 0.09 & 0.0375 & 0.0225 \\
\hline
\end{tabular}

\subsubsection{TYPICAL STOCHASTIC FORMULATIONS}

The two-stage stochastic programming problem where the first and second stage variables are considered together resulting in the deterministic equivalent (4.7), can be interpreted as the recourse problem (RP). In the RP the first stage variables are decided taking into account all possible scenarios, which enlarges the problem as much as scenarios are evaluated. A simplified approach is to consider each scenario separately, assuming the information on the each will be certain once the decision is to be made. Therefore, "perfect information" is assumed for each scenario and computing them separately and weighting the cost function by the corresponding $\pi\left(\xi_{i}\right)$ represents the best theoretical outcome in the long run (PI, a.k.a: wait-and-see). Finally, a second simplification neglects the randomness of the uncertainty and assumes it equal to its weighted average. As a consequence, the realizations of the second stage variables are fixed and the optimization problem becomes a regular deterministic problem, which determines the first stage variables. However, in reality the second stage will reveal all the scenarios in the long run, and at that point one will have to cope with the actual hydrogen 
demand and previously set hydrogen production. This solution is named the expectation of the expected value problem (EEVP), and is a usual simplification of the TSSP problem. A thorough discussion of these approaches and their value in addressing a real-world optimization problem considering stochastic uncertainty, including several examples, is provided by Birge and Louveaux (2011).

It is usually interesting to assess whether the two-stage programming stochastic offers an advantage over the two simplified approaches. For this purpose, Birge and Louveaux (2011) proposed the so called value of the stochastic solution (VSS) that is used in this study, as well as the expected value of perfect information (EVPI). The former quantifies the gain in the objective function resulting from considering the randomness of the uncertainty (i.e.: RP), versus its weighted average (i.e.: EEVP). The formula is presented in (4.11). The latter (4.12) compares the RP against a theoretical case where demand is certain and known beforehand (i.e.: PI), although this is not realistic.

$$
\begin{aligned}
& V S S=R P-E E V P \\
& E V P I=P I-R P
\end{aligned}
$$

\subsubsection{CASE-STUDY RESUltS}

Considering actual plant data from a DR solution (discussed in section 4.2.1 Models and data reconciliation), the TSSP solutions for the RP, EEVP and PI problem are shown in Table 4.4. The problem RP coded in $\mathrm{GAMS}^{\circledR}$ (GAMS Development Corporation, 2019) is solved with CONOPT (Drud, 1985, 1994, 2002). The problem involves 15958 variables and 14925 constraints, and is solved in $76.38 \mathrm{CPU}$ seconds (Intel ${ }^{\circledR}$ Core $^{\mathrm{TM}}$ i7 $2.50 \mathrm{GHz}$ and 16.0 GB of RAM). In terms of computational efficiency the results are suitable for the online application. Moreover, typical techniques of decomposition (see for reference: Li et al., 2012 and You and Grossmann, 2013) were dismissed as alternative formulations due to the satisfactory results of the monolithic RP formulation. In addition, the EVPI and VSS are presented in the same table to analyze the value of considering uncertainty explicitly. Due 
to confidentiality reasons, representative but fictitious prices of hydrogen costs and $H C$ loads are used in this study.

It is interesting to notice that with an EVPI of less than one percent it does not seem to be worth investing in additional information from hydrogen demand or light ends generation of the network. It should be considered that, more information it almost surely, requires equipment investment to undertake better analysis at the refinery laboratory or allocate more resources to the hydrocarbon cuts' properties predictions. However, the VSS shows an improvement of circa one order of magnitude compared to the EVPI, which is due to the incorporation of the stochastic uncertainty in the whole decision-making process from the beginning. In other words, if the uncertainty is estimated when deciding how much hydrogen should be produced and then corrected once the uncertainty reveals (i.e.: EEVP), the objective function is around ten k€ per hour worse than considering the uncertainty from the first stage (i.e.: RP). That is the "price" of simplifying the uncertainty when deciding on the hydrogen production, and neglecting the stochastic nature of hydrogen demand and LIG generation.

Table 4.4 - Results of the implementation of the TSSP formulation over the typical stochastic assumptions, i.e.: perfect information (PI), recourse problem (RP), expectation of the expected value problem (EEVP). These are used to calculate EVPI and VSS as suggested by Birge and Louveaux (2011). "Percentage w.r.t. EEVP.

\begin{tabular}{|l|l|l|l|l|l|l|}
\hline $\mathbf{P I}$ & RP & EEVP & \multicolumn{3}{|l|}{ EVPI } & \multicolumn{2}{l|}{ VSS } \\
\hline $\mathrm{k} € / \mathrm{h}$ & $\mathrm{k} € / \mathrm{h}$ & $\mathrm{k} € / \mathrm{h}$ & $\mathrm{k} € / \mathrm{h}$ & $\%^{*}$ & $\mathrm{k} € / \mathrm{h}$ & $\%^{*}$ \\
\hline 737.176 & 735.936 & 725.014 & 1.240 & 0.17 & 10.923 & 1.51 \\
\hline
\end{tabular}




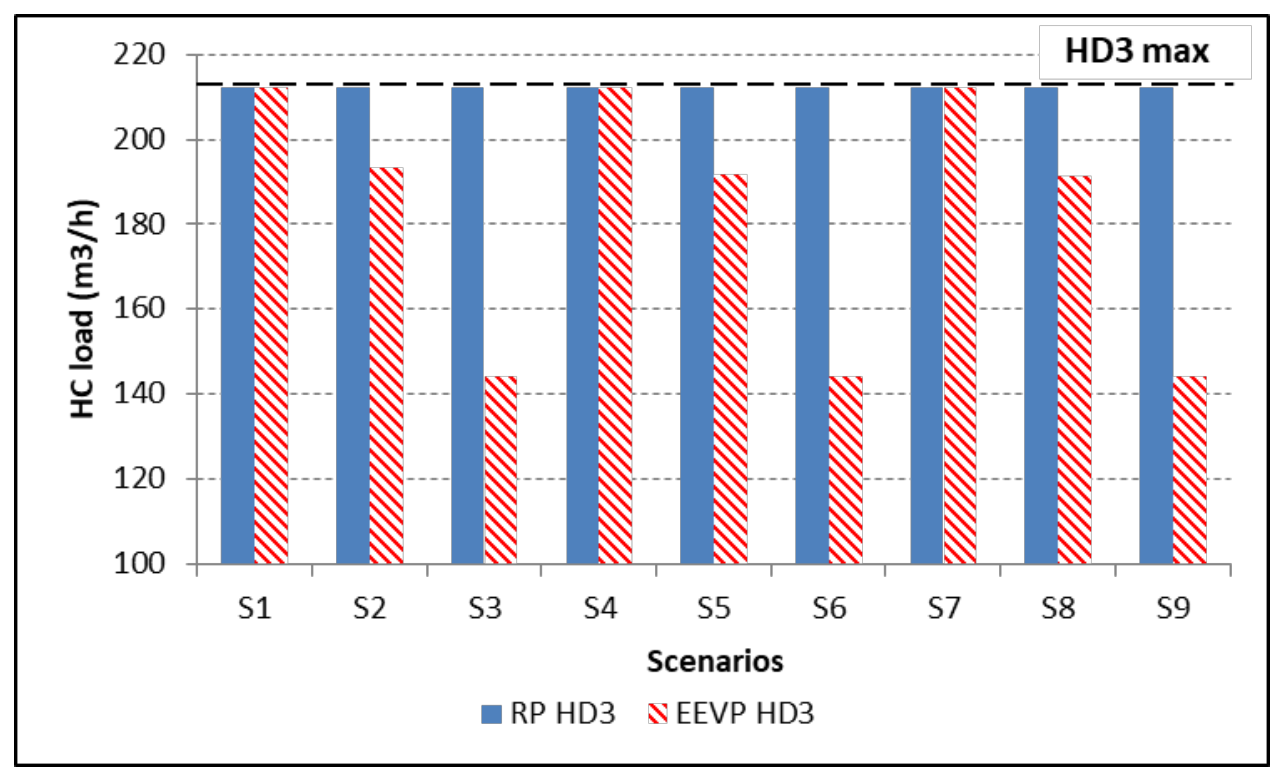

Fig. 4.11. RP and EEVP solutions for HC loads of process unit HD3.

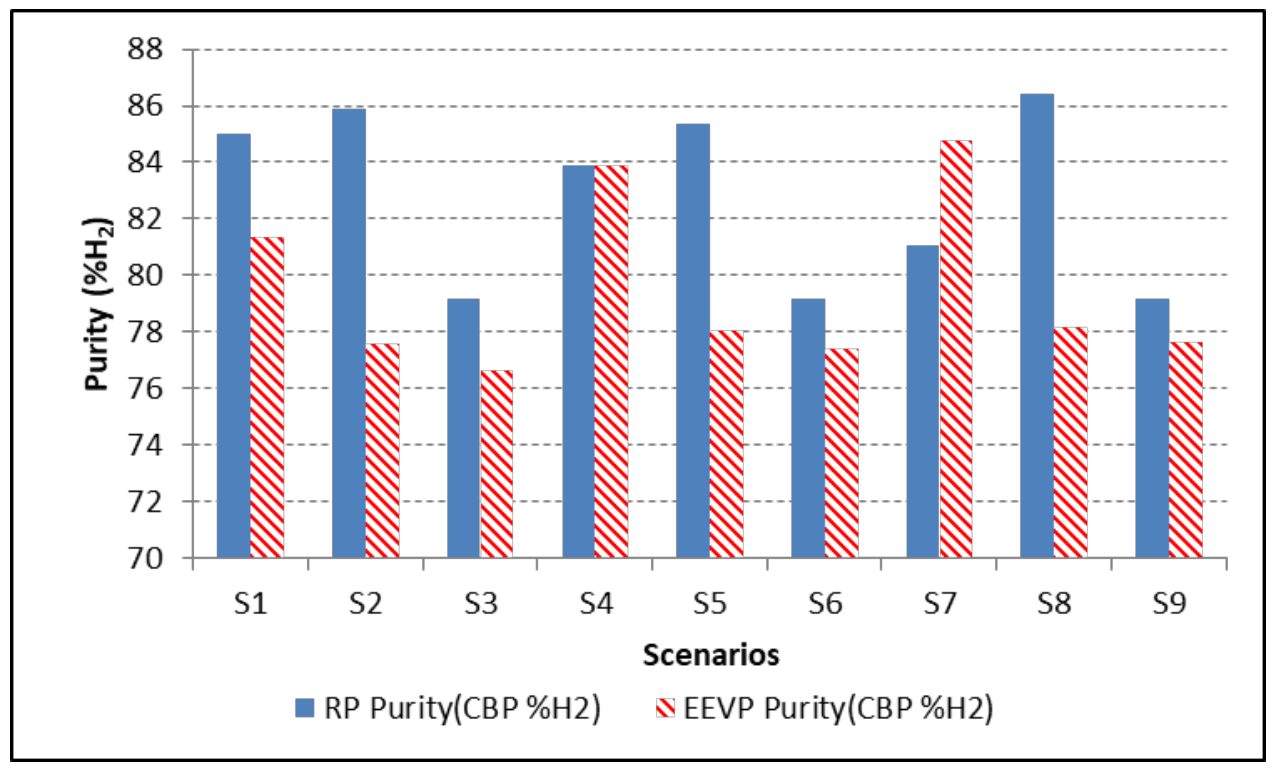

Fig. 4.12. Low purity header hydrogen purity at scenarios S1 to S9 applying RP and EEVP.

The same analysis applies when HC loads of EEVP and RP solutions are compared. For example, if the major hydrogen consumer is analyzed (i.e.: HD3) it could be seen how in most of the scenarios the RP outperforms EEVP (Fig. 4.11). The most favorable results for EEVP are at scenarios S1, S4 and S7, where HD3 maximum load capacity is reached. 
The rest of the scenarios require HC load to be below HD3 maximum to cope with hydrogen demands. However, RP is capable of meeting hydrogen demand at all scenarios without sacrifice of $\mathrm{HC}$ load. This translates directly to the objective function, where $\mathrm{HC}$ loads weight around 1000 times more than hydrogen production in volume (4.8).

In addition, RP solution improves LPH purity at all scenarios, except for S7 (Fig 4.12), which translates into more effective usage of recycled gases across the network contributing to economy of the process network. The particularity seen at S7 is related to the efficiency in the LPH purity management. This underpins in the concept that it only makes sense to hold high purity if that is required to satisfy $\mathrm{H}_{2}$ demand at reactors, for instance HD3 in this case. In other words, once the network demand has been met the best decision is to save hydrogen production costs. That is exactly the case of scenario S7, where $\mathrm{H}_{2}$ demand itself has not changed, only LIG generation (see Table 4.3). By not considering the stochasticity of the uncertainty the EEVP solution shows higher LPH purity (unnecessarily), impacting in the production costs negatively.

\subsection{CONSIDERING RISK IN THE DECISION MAKING PROCESS}

It should be remembered that, the previous approach (section 4.4) holds when the decisions do not take into account the risk associated to the objective function, a.k.a. riskneutral. Therefore, in the long run the expected valued $(\mathbb{E})$ is maximized regardless of the shape of the probability distribution of the cost function $(J)$. However, this does not give any insight on whether undesired realizations of scenarios are more or less likely to occur, since it is neutral towards risk and confidence level of realizations. Instead, a risk-averse formulation can be implemented in order to account for the risk of undesired maximum values of the cost function $J$ to be within a given probability. For instance, Fig. 4.13 illustrates the main differences in the shape of cost functions between risk-neutral (Fig. 4.13 - left) and risk-averse (Fig. 4.13 - right) formulations. The risk-averse chart presents the minimization of conditional value-at-risk (CVaR), which is a popular risk measure utilized in risk-averse stochastic optimization. In essence, the risk-neutral formulation 
presents the highest frequencies at low values of $J$ (see the red circles in Fig. 4.13) being the best $J$ on average. In contrast, the risk-averse formulation results in more a compact probability distribution compared with risk-neutral, and the best "tail" shape of the curve which translates into less frequent high values of $J$ (see the green circles in Fig. 4.13).

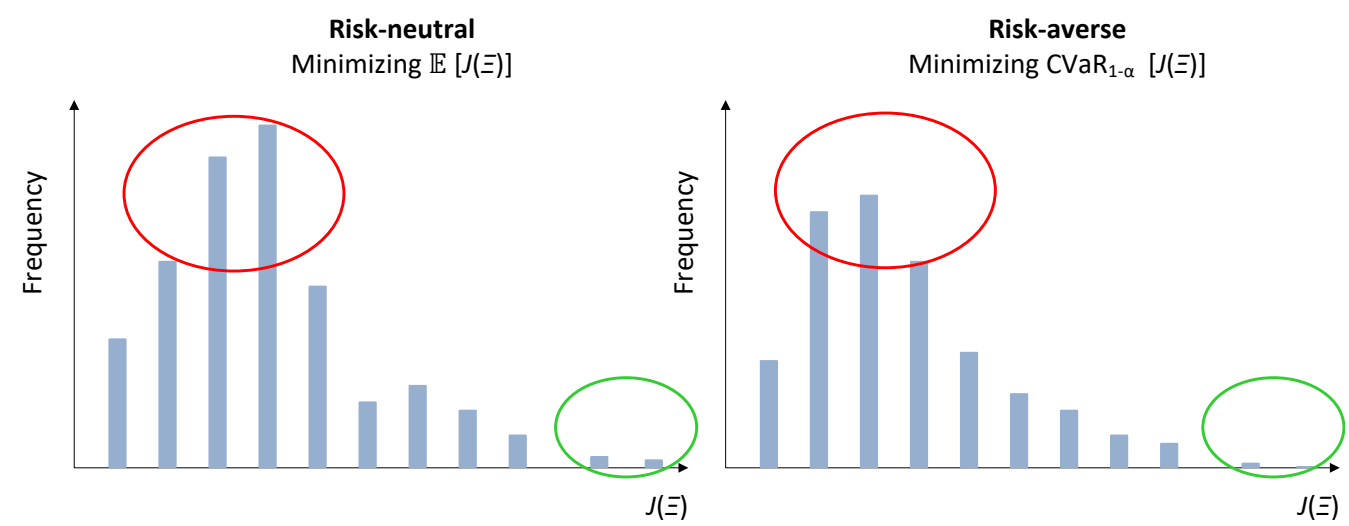

Fig. 4.13 - Left. Cost function J against frequency of occurrence resulting from a risk-neutral minimization of the expected valued $([/])$. Right. Cost function $J$ against frequency of occurrence resulting from a risk-averse minimization of the conditional value-at-risk $\left(\mathrm{CVaR}_{1-\alpha}[J]\right)$ at confidence level 1- $\alpha$.

In the remaining of this sub-section I analyze the formulation and results of applying a TSSP approach with a risk measure as objective function, hence this stochastic formulation is known as risk-averse. In particular, this study uses two risk measurements: value-at-risk (VaR) and conditional value-at-risk (CVaR), which are properly defined hereinafter.

\subsubsection{Conditional VALUE-AT-RISK}

First of all, it is important to present the definition of VaR as in (4.13). This risk measurement simply defines a value $\omega$ which is the least value of the random variable $\Xi$, where the likelihood of $J$ being less than $\omega$ is greater than or equal to a confidence level 1$\alpha$ (see Fig. 4.14). Another popular risk measure is CVaR defined as in (4.14), which corresponds to the average of the tail of the distribution of $J$ for values greater than VaR (see Fig. 4.14) and it is actually more useful in optimization for its convexity and other 
properties such as subadditivity (Pflug, 2000). Equation 4.15 shows how CVaR and VaR relate to each other, being trivial to see that $\mathrm{CVaR}$ is greater than VaR. More details on the characteristics of VaR and CVaR can be found in Rockafellar and Uryasev $(2002,2000)$, Uryasev and Rockafellar (2001) and Pflug (2000).

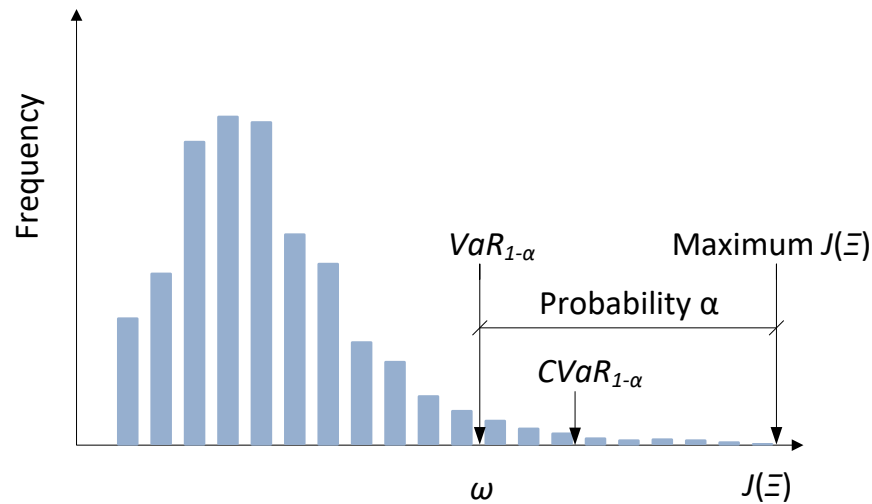

Fig. 4.14 - Graphic representation of $V a R_{1-\alpha}, C V a R_{1-\alpha}$ and $\alpha$ with respect to cost function $J$.

$$
\begin{aligned}
& \operatorname{VaR}_{1-\alpha}(J(\Xi)) \stackrel{\text { def }}{=} \inf _{\omega \in \mathbb{R}}\{\omega \mid P(J(\Xi) \leq \omega) \geq 1-\alpha\} \\
& \operatorname{CVaR}_{1-\alpha}(J(\Xi)) \stackrel{\text { def }}{=} \inf _{\omega \in \mathbb{R}}\left\{\omega+\frac{1}{\alpha} \mathbb{E}[J(\Xi)-\omega]_{+}\right\} \\
& \operatorname{CVaR}_{1-\alpha}(J(\Xi))=\operatorname{VaR}_{1-\alpha}(J(\Xi))+\alpha^{-1} \mathbb{E}\left[J(\Xi)-\operatorname{VaR}_{1-\alpha}(J(\Xi))\right]_{+}
\end{aligned}
$$

A practical formulation of the CVaR objective function is presented in (4.16.a-c), the full deduction is illustrated by Artzner et al. (1999). The right hand side of (4.16.a) eases the formulation of the minimization problem, which for all practical purposes represents the actual objective function minimized. Additionally, all previously explained constraints of the recourse problem (4.7.b-f, 4.9-b) remain unchanged and are imposed as well.

$$
\min _{u_{F}, u_{S}(\cdot)} C V a R_{1-\alpha}\left[J\left(u_{F}, u_{S}(\Xi), x(\Xi)\right)\right] \Leftrightarrow \min _{u_{F}, u_{S}(\cdot), \omega} \mathbb{E}\left[\omega+\alpha^{-1} \varphi(\Xi)\right]
$$

Subject to: 


$$
\begin{aligned}
& J\left(u_{F}, u_{S}(\xi), x(\xi)\right)-\omega \leq \varphi(\xi) \forall \xi \in \Xi, \\
& \varphi(\xi) \geq 0 \forall \xi \in \Xi,
\end{aligned}
$$

All constraints imposed in the RP formulation

Same hydrogen demand scenarios studied in the RP formulation

Therefore, the recourse problem of expected value (risk-neutral) is transformed into a risk-averse stochastic optimization problem using $\mathrm{CVaR}$, which sets the grounds for comparing and discussing both solutions. Table 4.5 shows the results for CVaR and VaR considering the same scenarios presented for RP at two confidence levels $1-\alpha$ (99\% and $95 \%$ ), and risk-neutral (i.e. $\alpha=1$ ). Notice that in this case the hydrogen problem is formulated as a minimization problem instead of a maximization as in the previous examples. This is only for practicality of formulation for the CVaR, and does not affect the reasoning behind the analysis.

Table 4.5 - Results of CVaR, VaR and hydrogen plant $\mathrm{H} 4$ at confidence levels $95 \%$ and $99 \%$.

${ }^{*}$ Percentage over total production capacity. ${ }^{* *}$ Intel ${ }^{\circledR}$ Core $^{\mathrm{TM}}$ i7 $2.50 \mathrm{GHz}$ and $16.0 \mathrm{~GB}$ of RAM. ${ }^{* * *}$ riskneutral solution (i.e.: $\alpha=1$ ), RP solution. ${ }^{+}$Not applicable.

\begin{tabular}{|c|c|c|c|c|c|}
\hline Confidence (1- $\alpha)$ & CVaR1- $\alpha$ & VaR1- $\alpha$ & \multicolumn{2}{|c|}{ H4 } & Time \\
\hline$\%$ & $k € / h$ & $k € / h$ & $N m 3 / h$ & $\% *$ & CPU** $^{*}$ \\
\hline 95 & 735.88 & 735.88 & 37884.06 & 86.10 & 71.46 \\
\hline 99 & 735.88 & 735.88 & 37884.06 & 86.10 & 43.74 \\
\hline $0^{* * *}$ & NA $^{\dagger}$ & NA $^{\dagger}$ & 37066.68 & 84.24 & 0.92 \\
\hline
\end{tabular}

According to Table 4.5 it could be deemed that changing risk from a confidence of 95\% to $99 \%$ changes very little the detriment in profit for the process, CVaR and VaR in all cases. Moreover, the same change of confidence level (95\% to $99 \%$ ) shows a negligible impact in the hydrogen production at $\mathrm{H} 4$, although it is around $800 \mathrm{Nm}^{3} / \mathrm{h}$ (about $2 \%$ ) higher than the RP solution, see Table 4.5. In other words, decreasing by five percent the risk of the network profit will be almost indistinguishable in terms of extra hydrogen 
production. Furthermore, it is important to notice how hydrogen purity in the low purity header (LPH) does not change very significantly (see Fig. 4.15), either. In this regard, the $\mathrm{RP}$ (risk-neutral) solution and $\mathrm{CVaR}_{0.01}$ present similar patterns of hydrogen purity across scenario, except at scenarios S5, S7 and S8, where absolute differences in hydrogen purity are: $0.72 \%, 5.1 \%$ and $1.1 \%$ respectively. Hydrogen distribution optimization is achieved by multiple changes in purification membranes, and internal streams in a number of headers that help source the most appropriate hydrogen purity and mass flow to each plant in order to satisfy its hydrogen demand for the $H C$ load. Therefore, a detailed breakdown of each scenario distribution solution is deemed out of scope. Instead, it is useful to understand more general patterns of each formulation.

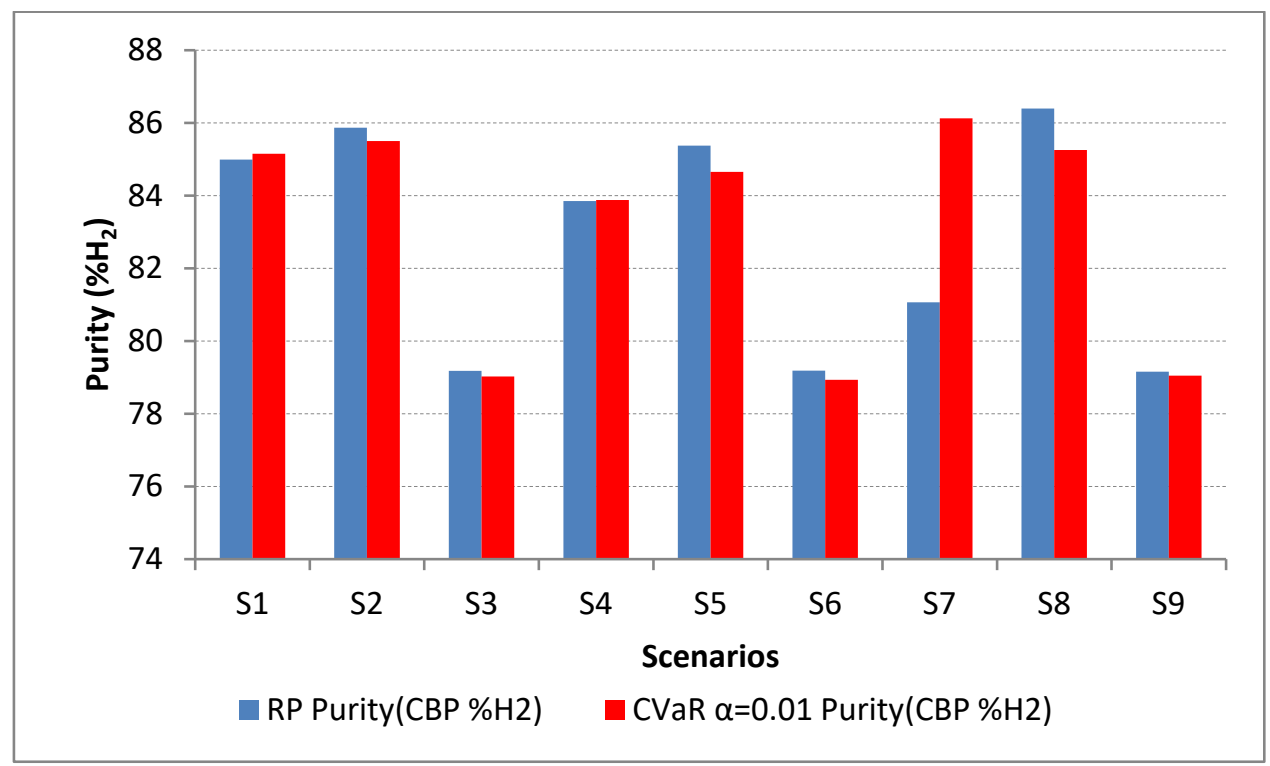

Fig. 4.15 - Low purity header (LPH) hydrogen purity at scenarios S1 to S9 applying RP and CVaR $\mathrm{R}_{0.01}$.

In essence, the RP solution copes with maximum $H C$ load at all scenarios with around $2 \%$ less fresh hydrogen production. However the profile of hydrogen purity in the LPH is similar for either solution with mixed differences, at scenarios S5 and S8 the RP operates at higher purities and at scenario S7 the $\mathrm{CVaR}_{0.01}$ operates at higher purities. It must be borne in mind that, $H C$ load to hydrogen consumer is at its maximum in all scenarios and confidence levels considered, therefore improvement of profit in scenarios 
should come from better hydrogen distribution and fresh hydrogen saving from $\mathrm{H} 4$. Certainly, this solution is case specific and greatly depends on the actual hydrogen demand circumstances.

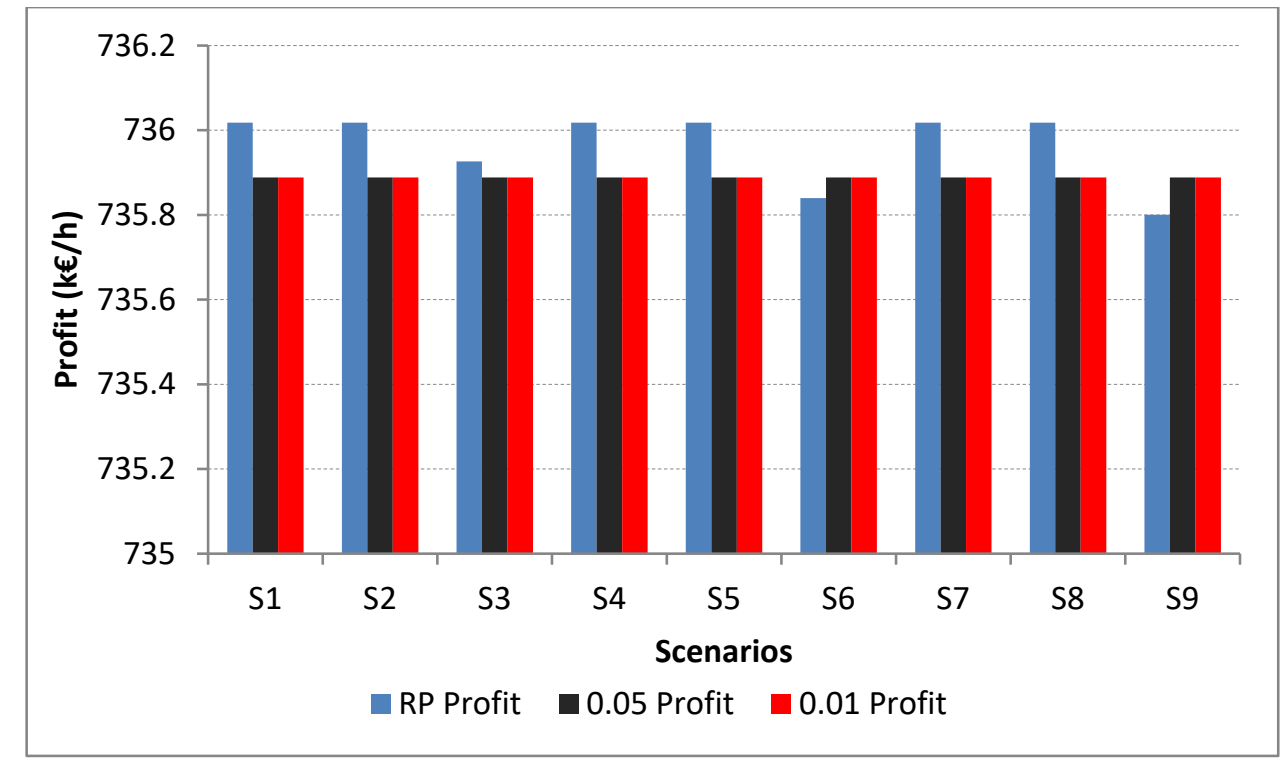

Fig. 4.16. Profit results over scenarios for RP (risk-neutral), $C \mathrm{CVR}_{0.05}$ and $C \mathrm{CVR}_{0.01}$.

An interesting point of view, is to compare profit at each scenario for CVaR and risk-neutral (i.e.: RP) solutions. Fig 4.16 presents those results. It is important to highlight that, considering risk (99 and 95 percent of confidence level) presents a more stable profit across scenarios, at the price of being less on average than the RP. In particular, scenarios six and nine are the ones that RP profit is less than CVaR profits. In the rest, RP profit is greater than CVaR profit. It must be borne in mind that, these figures are illustrative for the analysis, and not real in terms of profit amounts. Furthermore, the difference between profits is still very narrow and long term results should be analyzed for delivering a more robust discussion regarding the actual significance of these figures within the refinery business context. Certainly, the viewpoint and contribution of decision-makers, such as line managers, and business managers, is deemed of key importance in order for that analysis to be meaningful with respect to business impact for a certain risk level. 
In overall, the minimization of the weighted average cost of all scenarios considered in the RP does not stop the results obtained in a particular scenario to differ significantly from the optimized average, as the formulation does not include any constraint on the spread or variance of that cost function. To avoid this situation, a measure of the risk of obtaining a cost function significantly worse than the average can be the objective function instead. However, this so called risk-averse solution comes at the price of lower expected profit in the long run, as it was mentioned before (see Fig. 4.16).

\subsection{Conclusions}

This chapter presents the optimization and control system of a hydrogen network in Petronor crude oil refinery of Repsol group. It combines data reconciliation and RTO with the implementation of the optimal policies in a commercial DMC+ control system. The optimal policies appear as a set of targets to maximize or minimize within constraints in the LP layer of the DMC+ and are extracted from the analysis of the process and the optimization results proposed by the RTO. This way of implementing RTO has proven to be very effective and allows dealing with dynamics and disturbances as it is executed in realtime with the sampling time of the DMC predictive controller. In addition, the familiarity of the personnel with the DMC interface facilitates the adoption and use of the system and, being based on the DMC models, avoids the possible incoherencies with the ones of the RTO.

In addition this chapter studies the advantages of incorporating uncertainty explicitly in the decision making process aimed at managing unknown hydrogen demands created by processing of different crude oils. For this purpose, several scenarios were defined and Two-stage stochastic optimization was applied to the problem of optimal hydrogen distribution. In order to evaluate the improvement, two indexes were considered, the Expected Value of Perfect Information, EVPI, and the Value of Stochastic Solution, VSS. The former suggests that little gain is obtained by improving the knowledge 
on the quality (hydrogen demands) of the hydrocarbon loads being processed, but the VSS indicates that it may be worth to use the Two-stage stochastic optimization in the RTO. Although the results presented are for a particular two-hour period of time, similar conclusions are obtained when studying larger time periods. Finally, the use of an alternative objective function, risk of having a value of the cost function far from what expected, instead of the expected value over all scenarios was considered. More specifically, the Conditional Value-at-Risk, CVaR, was used. The results show a decrease in the cost function as expected. If the risk factor compensates this, is something that will require a deeper analysis, engaging business decision-makers at different levels in the organization in order for it to be representative of the actual business impact. 
5 DeCision Support NETWORK 



\section{Abstract}

Decision support frameworks (DSFs) are the natural environment of decisionmaking tools, however in practice these tools reside in different silos across multiple systems within company. This configuration challenges information and data exchange amongst tools in a transparent manner, which can result in inconsistent solutions due to different applications using different data sets for the same purpose. In order to address these issues and provide enhanced decision-making support across operations, a DSF architecture is proposed and studied in this chapter.

The DSF architecture features previously presented tools such as RTO and RTRS, while it introduces the data management system role, the digital twin role, online and offline simulation and other features. The discussion is focused on how the architecture would improve decision makers' capacity of making complex decisions supported by updated information from across the business, with especial interest in process operations. Furthermore, DSFs promote enhanced process knowledge and skills transfer, due to their ease-of-access to consistent information along with their forecasting and assessment capability over multiple operation alternatives (e.g. What-if analysis). In addition, the proposed DSF architecture considers tailored models, which are supported by the library of models under the scope of the digital twin.

Another relevant point in the analysis is about design criteria and recommendations with respect to developing a DSF. In this regard, consistency across applications, models maintenance and efficient data management are within the essential guidelines discussed. Thereafter, attention is given to enterprise-wide DSFs and how these may bring added value to business decision-making. In particular, DSFs give additional visibility towards strategic planning and process operations, backed on DSFs' simulation capability using online and offline data executed on multiple or even tailored models. 
Finally, I comment on challenges identified with respect to DSF deployment at industrial scale, which require further research. The last section summarizes the conclusions of this chapter. 


\subsection{DECISION SUPPORT FRAMEWORK}

In this section, attention is given to differences amongst support applications with respect to effective contributions towards betterment of decision making processes, especially under changing or uncertain conditions. Moreover, one significant contribution of this thesis is a study of the integration of different simulation and optimization techniques into one coherent decision support framework (DSF), whose features and architecture are discussed in detail hereinafter. It should be noticed that an actual case study analysis, with full integration of the tools presented herein, including a plant-wide RTRS and RTO, are out of scope of this thesis. Instead, a comprehensive qualitative discussion is provided which considers the major decision-making tools integrated in a decision support framework environment.

However the particular application, its integration into a decision support framework may improve its impact on final results, compared with the same scenario without integration. In addition, several DSF may be nested one into another depending on the scope of the application. As an example, Fig. 5.1 presents a generic enterprise-wide DSF block diagram (large scope), where a DSF for operation is contained (narrowed scope). It should be noticed that, this scheme is an expansion of the enterprise-wide DSF scheme presented in chapter 3 (Fig. 3.2). For instance, the DSF shown in Fig. 5.1 consists of six modules (numbered 1 to 6 ) and one data management system across the framework environment. Anyone module in this context refers to an application or set of applications within the DSF, which has an individual aim and provides a set of actions or intelligence to the user or another module. The box diagram in Fig. 5.1 shows the framework architecture along with its individual modules integration (e.g.: RTRS and AC\&O) and the main variables in the data management system.

Roughly, an enterprise-wide decision support framework as shown in Fig. 5.1 may integrate the following modules: 
- Maintenance (1 in Fig. 5.1), which deals with information typically relevant to assets maintenance. As an example would comprise condition monitoring (CM), maintenance planning and maintenance scheduling. This module contributes with sensible estimates on process equipment performance. Is fed with operating conditions or expected conditions, as well as production planning and supply chain information.

- General support (2 in Fig. 5.1), which deals with miscellaneous information flows across an organization. It would comprise information from diverse sources (submodules) such as suppliers, finance, customers, procurement and commercialization. This module contributes with data as expected demand of a product (from commercialization, and customer submodules) or replacement parts delayed (from supplier and procurement submodules) which are only examples of essential information sourced from general support module into the DSF. In return, it is fed from the data management system with updated figures for forecasting key supplies consumptions.

Enterprise-wide decision support framework

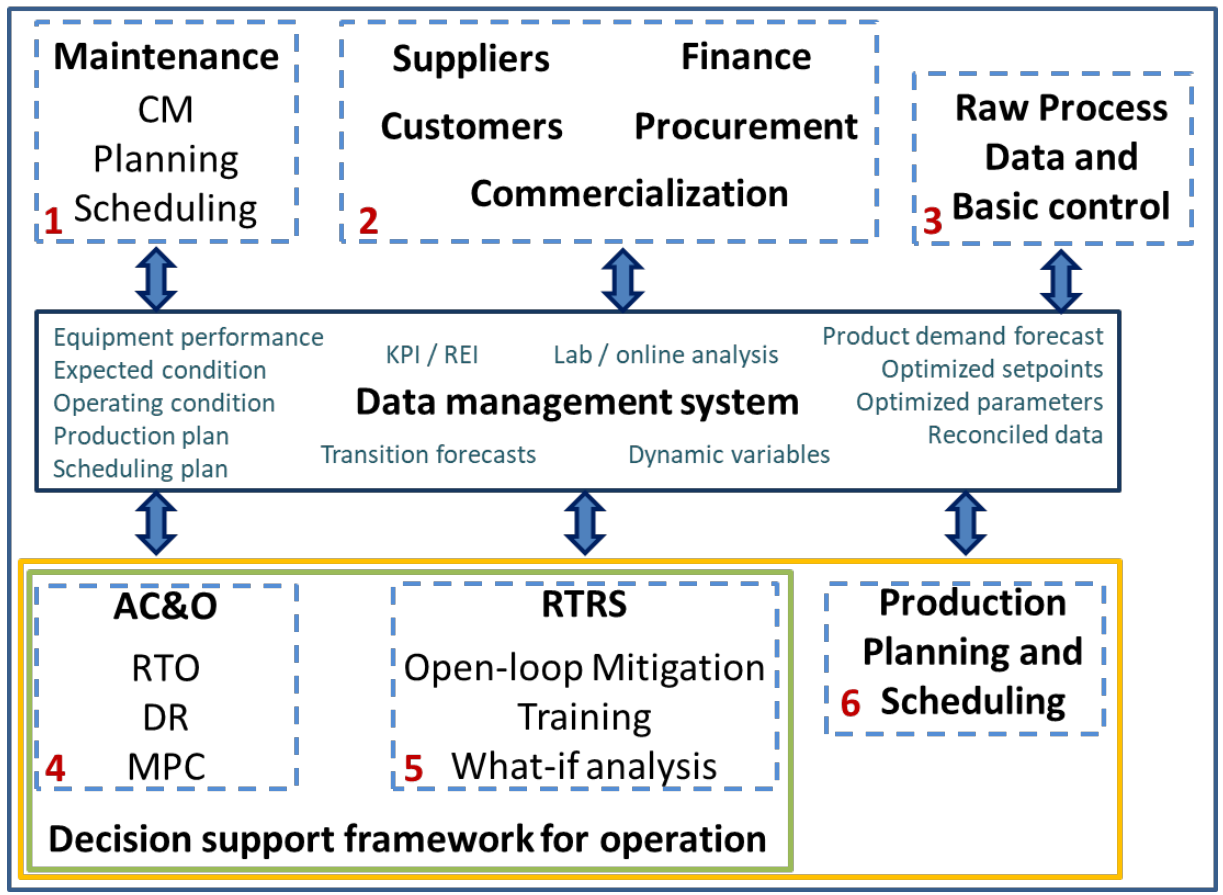


Fig. 5.1 - Example of decision support framework with six individual modules and data management. Modules four and five are further integrated into a decision support framework for operation (in green). Modules 4, 5 and 6 constitute production-oriented modules (in yellow) with close interactions with amongst them. AC\&O: advanced control and optimization. CM: condition monitoring. DR: data reconciliation. KPI: key performance indicators. MPC: model-predictive control. Lab: laboratory. REI: resource efficiency indicator. RTO: real-time optimization.

- $\quad$ Process data and control (3 in Fig. 5.1), which deals with raw process data and basic controls. This includes all instrumentation data, laboratory and online analysis, as well as basic control loops detailed information. This module contributes with process data required for operation and other modules. In return, data management would connect basic control with advanced control module which masters set points of key control loops.

- Advanced control and optimization (4 in Fig. 5.1), which is essentially at supervisory level in a control pyramid, where optimization routines are undertaken at some point in the workflow. This module comprises real-time optimization, data reconciliation and model predictive control (submodules). Therefore, it mainly contributes with reconciled data, parameter estimation and optimized set points. In return, is fed with raw data and process change of condition amongst other information from the rest of the modules.

- $\quad$ Real-time reconciled simulation (5 in Fig. 5.1), which deals with transition states and decision making process under uncertain changes. This module contains the three main submodules discussed on RTRS (chapter 3), i.e.: open-loop decision change mitigation, what-if analysis and training. Its contribution is in parameter estimations considering system dynamics, assessing transitions when RTO suggests significant changes in variables directions. This module is fed from reconciled values and raw data and online analysis as its main sources.

- Production planning and scheduling (6 in Fig. 5.1), which deals with optimization of production processes at timescales of weeks and days for planning and scheduling respectively. This module contributes with key production policies, which support RTO, MPC and RTRS cost functions, along with production plan data to support other modules. It would receive feedback from data reconciliation and RTO with updated 
process parameters and performance indicators. In the end, the relevant data is collected from the data management system to keep the values updated in the production planning and scheduling module.

\subsubsection{DECISION SUPPORT FRAMEWORK: ARCHITECTURE EXAMPLE}

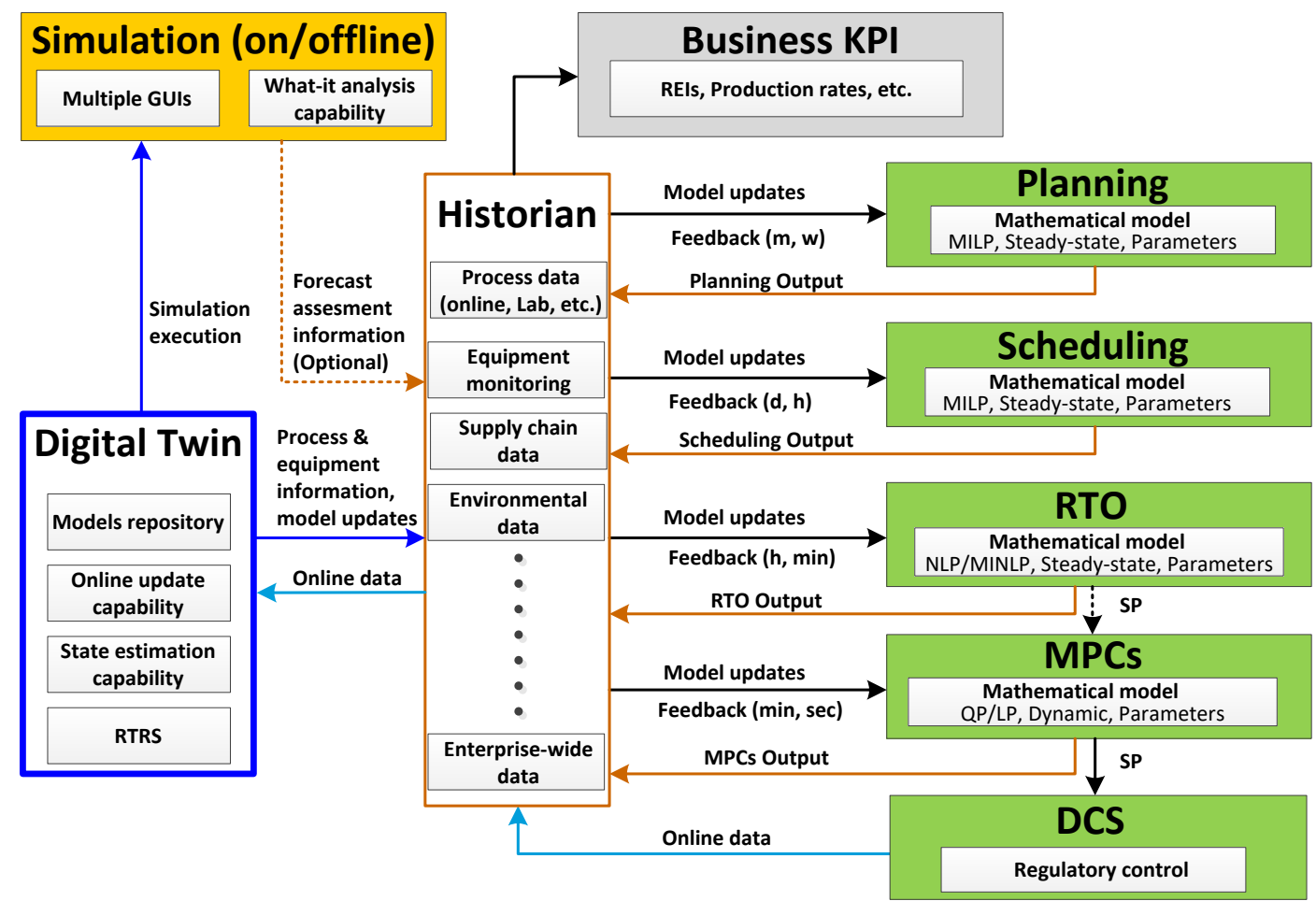

Fig. 5.2 - Example of decision support framework architecture with integration of control layers, simulation and optimization features. REI: resource efficiency indicator. KPI: key performance indicator. DCS: distributed control system. RTO: real-time optimization. MPC: model predictive controller. GUI: graphic user interface. SP: set points. QP: quadratic programming. LP: linear programming. NLP: nonlinear programming. MILP: mixed integer linear programming. MINLP: mixed integer nonlinear programming.

Decision support framework architecture centralizes data management, easing integration of applications and information exchange between levels as well as within same levels in the control pyramid. For instance, production-oriented modules (i.e. 4 to 6 in Fig. 5.1 - yellow rectangle) are of particular relevance in understanding information exchanges across the DSF and its architecture, since these have several levels of 
hierarchical dependency with data being managed directly between applications as already discussed in chapter 1 (revisit Fig. 1.2 and Fig. 1.5). For example, Fig. 5.2 depicts an example of architecture of DSF including simulation, optimization and control features. The example in Fig. 5.2 integrates production-oriented modules of the DSF (see yellow rectangle in Fig. 5.1) with one centralized data management system using plant historian database. In addition, the proposed architecture in Fig. 5.2 incorporates simulation and business KPI features to unleash the full potential of the DSF in aiding decision-making in process operation environments. In the following paragraphs, I describe how this DSF architecture (Fig. 5.2) enables seamless information and data exchange across individual applications enhancing decision-making capability.

Firstly, the historian plays the role of the data management system centralizing all sources of data, such as online process measurements, lab data, equipment monitoring data, and others (see Fig. 5.2). Furthermore, the historian collects key information (outputs) from traditional control and optimization applications (see green boxes in Fig. 5.2). These outputs are used automatically or on demand by other applications via historian, automated updates follow the feedback frequency required by each application (see feedback arrows in Fig. 5.2). In this architecture the historian is critical in keeping one data and information repository, which serves client applications requesting those data.

Secondly, the proposed architecture features a digital twin application, which is the root source of models of multiple natures (models repository in Fig. 5.2). In practice, operates as the library of models concerned to the decision support framework, especially those used in control and optimization tools (see green boxes in Fig. 5.2). Additionally, the digital twin itself executes automatic state estimation and model update routines using online data pulled from the historian. In fact, the RTRS would be considered within digital twin features to some extent, because it integrates state estimation with real-time simulation as discussed in chapter 3. Model updates, estimations and process equipment information resulting from the digital twin are available for all client applications within the DSF via historian. This architecture helps manage multiple mathematical models since 
these have the same source which simplifies consistent maintenance and human resource allocation for models maintenance.

Thirdly, this architecture features online and offline simulation with multiple graphic user interfaces (GUIs) and What-if analysis capability (see yellow box in Fig. 5.2). Users with different profiles can access multiple GUIs which allow them to run simulation experiments via digital twin. GUls should be assigned to users' profiles to reflect security clearance across users. For example, control room operators should have access to GUls which replicate DCS dashboards, while process engineers may have clearance to more detailed schematics (including chemical composition of streams, catalyst information, etc.). From GUIs, real-time simulation experiments can be run based on online current data, and RTRS. This provides enhanced forecasting capabilities, which generates results that can be available across the DSF, if necessary (see forecast assessment information, dashed arrow in Fig. 5.2). Additionally, control actions and other changes can be assessed using What-if analysis capabilities. For instance, optimization outputs from the RTO can be tested in search for simpler control action alternatives from an operating viewpoint with similar outcomes in terms of economic outcome (objective function). Refer to chapter 3 for a more detailed discussion and case study of What-if analysis in the context of RTRS.

Finally, business indicators are received via historian (see grey box in Fig. 5.2) in the Business KPIs feature, which help manager and executive level decision makers follow critical business trends with respect to process operations. This module comprises simple visualization tools such as dashboards and KPI charts.

The next sections discuss generic aspects common to DSF which are addressed from various viewpoints for completeness of the analysis.

\subsubsection{ENHANCED UNDERST ANDING OF THE PROCESS}

An effective combination of optimization and simulation fosters a better understanding of the process at all levels, which is essential for training and decisionmaking support. For instance, control room operators and engineers can conduct what-if 
analysis with actual plant data, based on normal operation instructions. This is helpful, for ease of illustration of newcomers to these positions. Moreover, for a decision to be successful it has to be underpinned on sound process knowledge, awareness of uncertainty nature of data estimations, to list some of the main features. In this sense, a decision support framework may be of significant importance, additionally contributing as a training tool for decision makers. Although, there are commercially available operators training systems (OTS), these will typically consider only previously validated data or scenarios, not current-time plant reconciled data. To the best of my knowledge, OTS providing current-time data neither reconcile nor use first-principle based dynamic models. Thereby, the proposed decision support framework may well be a strategic complement to traditional OTS platforms.

\subsubsection{IMPROVED DECISION MAKING}

Uncertainty sources are considered in the RTRS module (chapter 3) as disturbances of unmeasured parameters, and hydrogen demand and light-end generation within the optimization under uncertainty formulation (chapter 4). Therefore, users are able to address issues with or without consideration of process dynamics, always acknowledging the presence of uncertainties. Naturally, this leads to more educated and robust decisions. In practice, this framework incorporates explicit management of uncertainty, applying well-known techniques, aggregating flexibility in the analysis by addressing dynamic and stationary process network problems in the same suite. For instance, an operator may study hydrogen demand changes backed on RTRS for any given moment, and simultaneously apply stochastic formulation (risk-neutral or risk-averse). This is an example of a situation, where a decision support framework would be advantageous over other approaches.

\subsubsection{TAILORED MODELS}

Tailored dynamic models are simple to develop, which enhances applicability and model maintenance throughout operation. The reason for this is that, any final model 
structure resides fundamentally in the first-principles associated to each piece of equipment, which individually represent unitary processes such as reactors, separators, compressors and so on. These components are grouped in a library, which supports flexibility in developing any particular hydrogen network. Moreover, dynamic and stationary models are mutually compatible, therefore, from any one model the other is developed with ease. For instance, if a new process unit is under study, this should lead to a modification to the mathematical model. Essentially, this translates into arranging components from the library, then, connecting the process plant battery limits of the new plant to the network and generating the partition (as described in chapter 2). The new partition will show the mathematic model, from which the dynamic variables should be dismissed to update the stationary model. This is expected to become a convenient feature when commissioning new process plant, or modifications to existing ones. Hence, this approach is compatible with management of change (MoC) procedures, a wide spread business practice for coping with changes at all levels in an organization. In brief, MoC is an assessment of changes in an organization (permanent or temporary) which applied on process network operations requires forecasting impacts of those changes across assets. As mentioned, any infrastructure change in plants would potentially have an impact across models, which requires procedures in place for check listing that modifications are managed successfully. This might well be a MoC procedure, so a decision support network with the proposed modules fits in naturally.

\subsection{DESIGN OF A DECISION SUPPORT FRAMEWORK}

It is noteworthy that designing a decision support framework (DSF) such that all tools within the framework are effectively integrated, requires some basic criteria to be fulfilled. Apart from the concrete case studies discussed, it is of utmost importance the methodology itself, which its primary aim is to provide better decision support utilizing several applications contained in a common environment compared with these applications scattered across multiple environments. Therefore, it becomes relevant to develop a set of design criteria for this methodology to be utilized successfully. It must be 
borne in mind that these criteria arise in light of my research results (see chapters 2 to 4 , and publications), and personal experience onsite at Petronor (18 months, see Appendix D), and is not mandatory by any means when designing a DSF. It is in effect a set of design recommendations based on this research work outcomes. Nonetheless, any design criteria should be tailored and consistent with the aims of the application.

In general terms, recommended criteria expand the following design concepts:

- models consistency across DSF (e.g. use of digital twin as models repository, see Fig 5.2),

- use of a unique interface environment for all modules (e.g. historian),

- extensive use of data should be supported where possible (e.g. historian),

- capability of online and offline tools executions (e.g. digital twin).

The aforementioned are further described in the following section.

\subsubsection{RECOMMENDED DESIGN CRITERIA}

All models within the framework should be consistent with respect to their variables. Therefore, regardless of the detail level of each model, compatibility of the main variables should be assured. For example, in the hydrogen network case study, dynamics are acknowledged in the RTRS, the RTO does not account for those variables. However, steady state values of all variables are consistent one another. In this way, compatibility of both models is assured, facilitating their integration within the framework.

Interface of modules across the framework should be unique. Although computation of modules is entirely independent from one another, results should be interchangeable on the appropriate platform. For example, MS Excel ${ }^{\circledR}$ is used in the proposed DSF (Petronor case study) as common interface along with the plant historian, $\mathrm{PI}$ (Osisoft), refer to chapters 2 to 4 for more details. The concept is summarized in Fig. 5.3, where the interface layer is in yellow. It is highlighted the fact that the interface layer is the same software environment for modules and users. However, interface with users 
does not bring editing or visualization capabilities in addition to those embedded in the historian (typically, this have simplified schematics of process units). Hence, GUIs are still required for detailed studies as what-if analysis and real-time simulation, as explained in section 5.1.1 (Fig. 5.2).

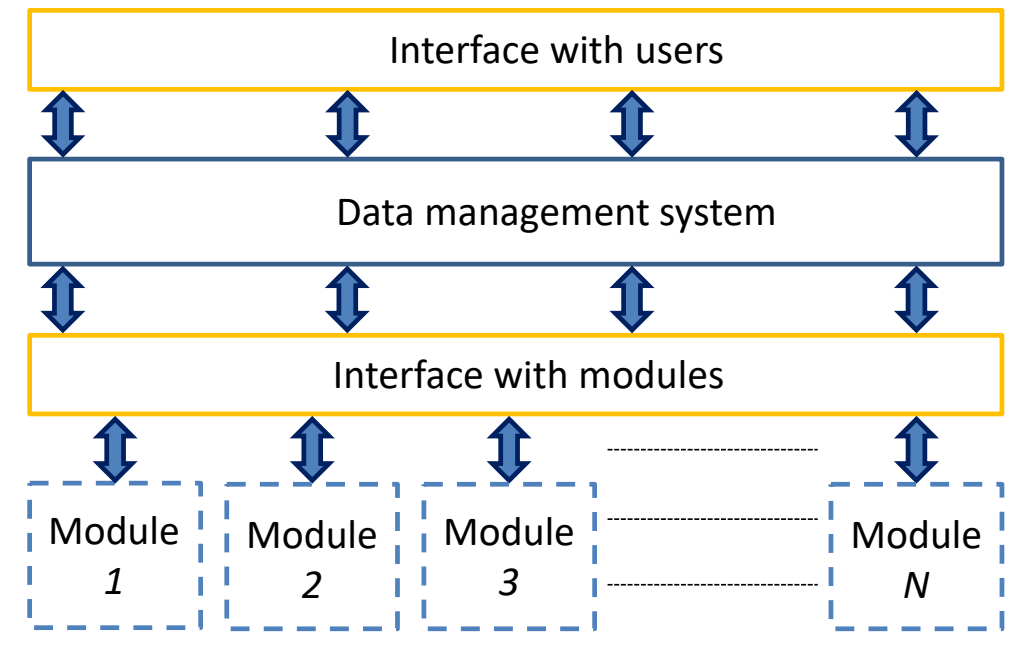

Fig. 5.3 - Layers of communication within a DSF with $N$ modules.

A DSF should make extensive use of plant data, including: equipment condition, advanced control input and output data, planning and scheduling information, amongst others. Data feeds and it is produced by modules across the framework, therefore a DSF should take into account sufficient data management capacity.

A DSF should be able to operate either based on current or past data, enabling customized scenarios to be added into the analysis. This feature is particularly useful in troubleshooting, operational training of newcomers and general purpose what-if studies. Examples of current and past data case studies are mostly discussed in chapter 3 (RTRS), while customized scenarios solved via two-stage stochastic programming (risk-neutral and risk-averse) are presented in chapter 4.

Industrial stakeholders are already envisioning modular standard-based distributed control system (DCS). These are interoperable and include programmable logic controller (PLC) modules in its architecture. Essentially, this approach benefits from a common 
standard for communication amongst users in the process control environment from levels 1 to 4, enabling unlimited modules as long as these use the same standard in what is called open connectivity framework (OCF). One of the challenges faced by this approach is how to address existing control and monitoring systems, without requiring upgrading of control systems across process units which may be big hurdle to overcome by companies. Additionally, it remains unclear how features as digital twins and simulation are integrated in this standard-based architecture for process control. Actual examples are still in research and development stages at the moment, being the most prominent examples: Open Process Automation ${ }^{\mathrm{TM}}$ Forum (OPAF, 2018) and the User Association of Automation Technology in Process Industries (NAMUR, 2018).

To the best of my knowledge, there is not yet any widely proven commercially available platform which satisfies the design criteria of the proposed decision support framework in its entirety. However, I acknowledge that most likely major vendors are progressing fast in this direction (ABB, 2018; Beck, n.d.; KBC, 2018), especially, in the fields of data analytics and decentralized computation (e.g. Cloud processing). Moreover, vendors of modelling and optimization solutions to the process industry would likely comply with all the criteria if their products features are aggregated and considered altogether. In this regard, commercial tools would pass on to the user the integration of different modules, and thereby actual advantages of a framework approach would not hold.

\subsection{ENTERPRISE-WIDE DECISION SUPPORT FRAMEWORK}

A particular interest is given to DSF applications aimed at integrating modules across an organization including a wide range of activities such as supply chain, process operations, procurement, maintenance, commercialization, amongst others. These are called enterprise-wide DSF (see Fig. 5.1) and have particularities that are noteworthy, such as:

- tailored information, in order to match decision makers with relevant indicators, 
- reinforcement of decisions under changing conditions, aiding policy decision makers in long-run and short-run decisions,

- fully informed decision, integrating most relevant and up-to-date information across all modules in a DSF.

In this section further discussion focused on the contribution of enterprise-wide DSF is addressed based on the aforementioned design recommendations and previously discussed case studies, along with their simulation and optimization decision support tools (for details refer to chapters 2 to 4 ).

\subsubsection{TAILORED INFORMATION}

Enterprise-wide decision support frameworks take into consideration users' profiles when showing information, matching decision makers with relevant information sets according to their respective hierarchical level. Thus, risk of excess and misleading information is reduced, while keeping all parties sufficiently informed. For instance, at operation level decision maker would be able to access all the information of DSF for operation (see Fig. 5.1), in addition to the most relevant variables or indicators of other modules such as demand forecast, equipment performance and production plan. In contrast, a decision maker in a finance role would require access to a complete different information set such as resource energy indicators, demand forecast, sales and production plan. An enterprise-wide DSF eases the integration of all data sources into a single historian database. Moreover, this database is accessible for all registered users to their corresponding hierarchical level and profile.

Additionally, DSF architecture enables modules to be incorporated or removed with ease. This is of special interest due to rapid business changes to be reflected promptly in the DSF keeping it up-to-date from a structure viewpoint. For example, if a new fuel quality is incorporated into the products of a refinery this is likely to have an impact in the hydrogen network, as well as in maintenance, demand estimation, among other variables. Equally important would be to review whether it makes sense to 
incorporate another module into the framework, for instance one related to government regulatory agencies monitoring such as environmental sensitive data and the like. All these alternatives are of simple design within this DSF architecture.

Although an integration of enterprise-wide information in a database poses all the aforementioned advantage, it faces some challenges as well such as data protection and security. In this respect, firewalls and cyber security features should be duly noted, since expanding systems integration it comes at the cost of higher exposure to potential threats. This is of particular sensitivity when integrating modules from external stakeholders, such as customers and suppliers. Further research and discussion on these challenges are out of the scope of this thesis and are open issues on the implementation of enterprise-wide DSF.

\subsubsection{REINFORCEMENT OF DECISIONS UNDER CHANGING CONDITIONS}

A thorough assessment of condition changes aids decision makers across the organization to take actions in a timely and orderly fashion more effectively. In this regard, what-if analysis based on RTRS (see chapter 3) helps assess scenarios based on current plant conditions (online data), and stochastic real-time optimization (see chapter 4) helps assess optimal decisions, risk-neutral and risk-averse, based on scenarios likelihoods. Changes across variables, parameters and data are regularly updated into the data management system. This enables quick incorporation of most up-to-date information across all data clients, which leads to awareness of propagation of changes across the organization. Furthermore, impact of changes can be assessed quantitatively across all modules, reinforcing the decision making process.

These capabilities are backed on effective enterprise-wide integration of modules, alongside simulation and optimization based tools. Actually, combining dynamic simulation with steady state optimization, and efficient online data exchange, provides a vast set of opportunities for decision support, especially under changing conditions. For instance, in the case study presented in chapter 4, a stochastic RTO may well have 
updated plant costs of hydrogen and hydrocarbon product prices. Hydrogen demands can be set either constant (from data reconciliation) or associated to a likelihood of occurrence (form historian and crude assay data). Simultaneously, RTRS unleashes the potential of testing several policies, particularly starting from online data, giving an additional support to RTO steady state based solutions. Moreover, if these results are propagated into other modules would impact on condition monitoring of equipment, planning and scheduling, to name a few. The main concept in this regard is how useful is a DSF setup to convey impactful figures to decision makers, bringing into consideration upto-date pieces of information that would have been unknown or overlooked otherwise. Likewise, uncertainties become naturally processed within the DSF either in the stochastic optimization module or RTRS, then propagated into any other relevant module variable. This updating mechanism via data management system, would underpin better forecasting features such as prognosis in maintenance modules, or plant cost estimations.

Ultimately, improvements driven by enterprise-wide decision support frameworks would derive in higher awareness of impacts across modules of unexpected conditions. Simply due to accumulation of experience, where decision makers develop better understanding of interactions and propagation of changes across all business levels. This awareness, in addition to the stochastic optimization and simulation of scenarios, would better support long and short term decisions.

\subsubsection{FULLY INFORMED DECISION MAKING}

In essence, sustained and consistent decision making processes aiming at coping with unexpected condition changes in process industries require the best up-to-date information tailored to meet these needs at all business levels within a company. Therefore, reflecting on the role and impact of accurate information is of key importance in aiding stakeholders in their decision making process. For the purpose of this discussion, information and data should be sharply distinguished in the following manner. Information, is a piece of knowledge represented quantitatively by a value or set of values typically emerged from data processing, e.g. reconciled data, performance indicators and 
demand estimation. Data, are the raw measurements, including laboratory analysis, e.g. purity, flowrates, temperature. Then it should be noted that, even when data is available what is really key in the decision-making process is the information provided, rather than data alone. In first place, accuracy of information requires some form of data reconciliation, which at its very least should account for instrumentation errors, standard deviation, faulty instruments and other measuring errors. Thus, plant raw data is converted, through the DSF, into reconciled data making it more valuable for decision makers across the business.

Reconciled figures serve as inputs for further processing into indicators relevant to each DSF module such as KPI or REI, or optimization based tools (planning, RTO, scheduling), unleashing decision makers full potential. In fact, DSF contributes towards a fully informed decision making process, meaning that all relevant information is available for the right decision maker to consider it. This includes recommendations from optimization based tools, which typically would assess a problem from a specific viewpoint giving its mathematical optimum. However, mathematical models used in optimization have certain degree of misrepresentation of actual processes, resulting in their solutions subject to further assessment by a relevant decision maker. In spite of that, DSF ease the decision making process by naturally bringing all relevant data and information into consideration in a timely manner. Therefore, updates of intermediate variables are automatically linked to their respective variable in the calculation cascade, or simply used as feedback of iteration loops.

In any case, DSF allow decision makers to account for information that would have been hidden somewhere in the raw data set, providing better quality of information as well as widening its sources. Particularly, these are advantageous in assessing enterprisewide impacts of decisions as such.

Some of the challenges towards fully informed decision support are related to efficient data exchange across modules. In addition, the incorporation of data from outside a common DSF are the most likely to blame for diminishing the overall data 
exchange performance. Likewise, other authors present similar ideas such as integrated model-centric frameworks (Rolandi and Romagnoli, 2010) or model integration within digital twins (Vrabič et al., 2018), which focus their attention on framework architectures to optimize DSF data exchange efficiency throughout the application lifecycle. Therefore, it may be as critical to efficiently integrate data sources across the business, in order to make information available, as it is the data processing itself that produces such decision support information (e.g. data reconciliation, performance and efficiency indicators). As an example, a DSF would be unsuccessful if most individual modules work properly and information is not available across the company to stakeholders, exactly the opposite should be aimed.

Another critical obstacle to overcome relies on the fact that state estimation of process models is rarely meant to run in real time with current plant data as starting point for real-time simulation, RTRS is an exception to this. In this sense, some steps forward have been taken with so called digital twins, although these tools are yet in an early stage in the process industry (Perino, 2019). Even though a digital twin of process plant is not yet well defined, it can be loosely conceived as a computer-based process or asset replica. The definition may vary slightly depending on the field of implementation, for instance in through-life engineering is defined as (Vrabič et al., 2018): "A digital twin is a digital representation of a physical item or assembly using integrated simulations and service data. The digital representation holds information from multiple sources across the product lifecycle. This information is continuously updated and is visualized in a variety of ways to predict current and future conditions, in both design and operational environments, to enhance decision making" (Erkoyuncu et al., n.d.). Therefore, a validated mathematical model (or a library of models, as described in section 5.1.1) may well be considered as a digital twin, as long as it provides accurate enough simulation values based on actual plant state at any given time. For instance, digital twins might well fit in individual modules, or sets of modules, within an enterprise-wide DSF enhancing the support to decision makers. An example of digital twin used within a DSF of productionoriented modules is shown in Fig. 5.2. I am confident that DSF and digital twins are 
complementary to one another, both contributing in decision-making under changing circumstances.

\subsection{CONCLUSIONS}

Decision support frameworks provide a common environment for information exchange of different decision tools. This is discussed, introducing the example of an enterprise-wide DSF with six individual modules (Fig. 5.1), briefly presenting each one. Furthermore, the architecture of production-oriented modules is described and analyzed in more detail (Fig. 5.2) as an example, which features digital twin (models repository), historian (data management system), simulation (online and offline), business KPIs (executive management dashboards) and process operation applications (planning, scheduling, RTO, MPCS, DCS). Based on these analyses, DSF provides advantages over individual modules in:

- understanding of the process, especially for newcomers (e.g. RTRS),

- decision-making process, due to broader availability of alternatives (e.g. RTRS, stochastic optimization),

- tailored models, due to flexibility in updating plant model flowsheet based on the dynamic library

In order to succeed in developing a DSF specific criteria with recommendations of design are presented, addressing the following aspects:

- models consistency across DSF,

- use of a unique interface environment for all modules,

- extensive use of data should be supported where possible,

- capability of online and offline tools executions.

Additionally, enterprise-wide DSF is discussed in further detail, finding the following as its most important features towards aiding decision makers at all levels: 
- $\quad$ ease of tailored information, matching decision makers with relevant indicators,

- $\quad$ multiple support in process changing conditions, aiding policy decision makers in longrun and short-run decisions,

- fully informed decision, integrating most relevant and up-to-date information across all modules in a DSF.

Finally, challenges in developing efficient DSF are identified and briefly discussed. For instance, it is of key importance to exchange data efficiently across modules, which increases its difficulty with the amount of modules in the DSF. Another open issue is an effective integration of digital twins in DSF, which in principle seems very promising, though not properly studied yet. 


\section{CONCLUSIONS \& CONTRIBUTIONS}





\subsection{SUMMARY OF CONTRIBUTIONS}

In this thesis I presented a study about the implementation of simulation and optimization techniques for decision support of process networks considering uncertainty, using Petronor crude oil refinery hydrogen network as case study. The principal contributions of this thesis are summarized in a fourfold, consisting of:

- Development and study of a dynamic simulation library of crude oil refinery hydrogen network components, which enables flexibility and ease-of-use towards building tailored hydrogen network models. An actual crude oil refinery hydrogen network simulation is briefly described since its model has been successfully built using the main dynamic simulation library. This topic is addressed in chapter 2;

- Development and study of an application of real-time simulation, called real-time reconciled simulation (RTRS), based on the dynamic simulation library and tailored to represent an actual process unit of Petronor refinery. The architecture of RTRS and how it exchanges information with other advanced control tool in the refinery, such as RTO and the plant historian, are discussed as well. This topic is addressed in chapter 3;

- Development and study of stochastic RTO (risk-neutral and risk-averse), considering hydrogen demand uncertainty across the process network. This model and formulation enhances a pre-existing deterministic RTO in Petronor refinery (case study). This topic is addressed in chapter 4;

- Description and study of the architecture of decision support frameworks which features multiple simulation, optimization, monitoring and control tools all with a common data management system (process plant historian). General enterprise-wide DSF along with production-oriented DSF are presented with examples This topic is addressed in chapter 5 .

\subsection{SUMMARY OF CONCLUSIONS}

The main conclusions of this thesis are summarized hereinafter: 
- complex process network arrangements can be efficiently translated into mathematical models and used for simulation or other computer-based tools using first-principle dynamic simulation libraries. The dynamic library developed is essential for this purpose, supporting stand-alone simulation models, in a semi-automatic manner, enabling its utilization in real-time reconciled simulation (RTRS);

- real-time reconciled simulation (RTRS), combines dynamic state estimation techniques (MHE) into a simulation environment, which enables online data usage and input of manipulated variables for assessing future conditions. Moreover, the proposed RTRS architecture provides decision support for operators, which is demonstrated through evaluation of what-if analysis on two case studies: an individual process unit (case I) and a process network (case II);

- RTRS methodology provides modelling versatility and ease of communication, complementarity with current plant-wide control systems (RTO / MPC) and intuitiveness of implementation. This aspect is central to further study the integration of RTRS, RTO and MPC in a common decision support framework;

- $\quad$ stochastic RTO, effectively incorporates explicit uncertainty in the decision making process to address unknown hydrogen demand as a consequence of processing different crude oils. Furthermore, two-stage stochastic optimization is successfully applied to the problem of optimal hydrogen distribution considering risk-neutral and risk-averse formulations. Although it has been demonstrated the additional value of formulating the stochastic problem with a risk-neutral approach, risk-averse results do not present significant advantages over risk-neutral with the data set studied;

- the proposed architecture of DSF features a digital twin tool which hosts the RTRS and library of models, a simulation tool with multiple GUls linked to users' profiles and control and optimization tools used for planning, scheduling, advanced control (e.g. RTO, MPC) and basic control (e.g. DCS, PLC), along with the historian (data management system) comprise the backbone of the proposed DSF for process operations; 
- decision support frameworks (DSFs) provide a common environment for information exchange of different decision tools, which has multiple advantages since integrates individual modules information enhancing decision-making process efficiency. Some identified advantages are:

- better understanding of the process, especially for newcomers. DSF are capable of online and offline simulation using online process data, hence control actions and abnormal operation can be assessed in a safely manner (e.g. RTRS, What-if analysis).

- data and information are managed by a dedicated data management system (e.g. historian), which enables efficient communication across applications within the DSF,

- decision-making process, due to broader availability of alternatives (e.g. RTRS, stochastic optimization),

- tailored models, due to flexibility in updating plant model flowsheet based on the dynamic library;

- considering enterprise-wide DSF the following are its most important features towards aiding decision makers at all levels:

- ease of tailored information, matching decision makers with relevant indicators (e.g. Business KPIs),

- multiple support in process changing conditions, which integrates the most relevant and up-to-date information across all modules in a DSF, aiding policy decision makers in long-run and short-run decisions.

\subsection{OPEN ISSUES}

Although the aforementioned conclusions are promising in terms of enhancing the utilization of optimization and simulation-based decision support tools, I have identified open issues that should motivate further research. Hereinafter a concise description of these is pointed out. 
Improve integration of state-of-the-art simulation platforms and optimization routines. Currently, full space discretization of models is not accessed from simulation platforms which typically rely on their own integration method with sensitivities calculations incorporated. However, the optimization engines require direct access to first and second derivatives at any given time to use their full potential, especially in large scale problems. Simultaneous full space discretization based on orthogonal collocation has proved to be efficient in general when combined with automatic differentiation of equations. Therefore, integration of both in the same platform would unleash simulation and optimization advantages altogether.

Modules maintenance throughout the application lifecycle is a key task for success. Even though many efforts in research have been focused on actual application performance as decision support tool, most of the challenges posed by an additional complexity of maintaining all the modules and models up-to-date has not been solved yet. Although this is expected to be somehow addressed by practitioners more than by academics, it still may need more attention for the sake of the use in actual processes.

Nonlinear stochastic optimization is heavily based on meaningful scenarios, which might not be easy to validate or even to be agreed when facing an uncertain condition. At the same time, more scenarios come at the cost of more computation time. Thus, some guidelines for scenario building and reduction may be incorporated for ease of use of the tool. In addition, nonlinear nonconvex problems are well solved to the level of local optimum, this should be born in mind at the time of implementing solutions. Most likely, users should reflect on the solution values seeking the root cause of major changes before actually implementing them. The main concern to be aware of is that depending on the starting point, the local optimum may be far from the global and this may lead to erratic or less robust solutions over a period of time. According to my experience and the case studies presented, once the root cause of the change proposed by the optimizer is clear the solutions are consistent given similar plant conditions. 


\subsection{PUblished CONTRIBUTIONS}

Published contributions of this thesis are listed hereinafter for reference.

\section{JOURNAL PUBLICATIONS}

- Galan, A., de Prada, C., Gutierrez, G., Sarabia, D., Gonzalez, R. (2019) Implementation of RTO in a large hydrogen network considering uncertainty. Optimization and Engineering. [Online] Available from: doi:10.1007/s11081-019-09444-3.

- Galan, A., de Prada, C., Gutierrez, G., Sarabia, D., Gonzalez, R. (2020) Real-time reconciled simulation as a decision support tool for process operation, submitted to Journal of Process Control

\section{CONGRESS PUBLICATIONS}

- Galan, A., De Prada, C., Gutierrez, G., Gonzalez, R. (2017) Dynamic simulation applied to refinery hydrogen networks. XXXVIII Jornadas de Automática, Gijón. In: [Online]. 6 September 2017. Available from: doi:10.5281/ZENODO.1013258 [Accessed: 16 May 2019]. Poster presentation.

- Galan, A., de Prada, C., Gutierrez, G., Sarabia, D., Gonzalez, R. (2017) Resource efficiency indicators usefulness for decision-making process of operators: refinery hydrogen network case study. In: Antonio Espuña, Moisès Graells, \& Luis B T Computer Aided Chemical Engineering Puigjaner (eds.). 27 European Symposium on Computer Aided Process Engineering. [Online]. Elsevier. pp. 1513-1518. Available from: doi:10.1016/B978-0-444-63965-3.50254-3. Oral presentation.

- Gutierrez, G., Galan, A., Sarabia, D., de Prada, C. (2018) Two-Stage Stochastic Optimization of a hydrogen network. IFAC-PapersOnLine. [Online] 51 (18), 263-268. Available from: doi:https://doi.org/10.1016/j.ifacol.2018.09.310. Poster presentation.

- Galan, A., de Prada, C., Gutierrez, G., Sarabia, D., Gonzalez, R. (2018) Simulación predictiva para el apoyo a la toma de decisiones de operadores de redes de procesos considerando incertidumbres. IV Simposio CEA de Modelado, Simulación y Optimización. Valladolid, Spain. ISBN: 978-84-697-8853-0. pp 88-89. Available from: 
http://albergueweb1.uva.es/smso2018/files/actas IVsmso.pdf [Accessed: 04 March 2020]. Oral presentation.

- Galan, A., de Prada, C., Gutierrez, G., Sarabia, D., Gonzalez, R. (2018) Validation of a hydrogen network RTO application for decision support of refinery operators. IFACPapersOnLine. [Online] 51 (18), 73-78. Available from: doi:https://doi.org/10.1016/j.ifacol.2018.09.256. Oral presentation.

- Galan, A., De Prada, C., Gutierrez, G., Sarabia, D., Gonzalez, R. (2019) Predictive Simulation Applied to Refinery Hydrogen Networks for Operators' Decision Support. IFAC-PapersOnLine. [Online] 52 (1), 862-867. Available from: doi:10.1016/j.ifacol.2019.06.170. Oral presentation. 


\section{REFERENCES}





\section{LIST OF REFERENCES}

ABB, 2018. Transforming chemical operations through technology Part 1: Using digital to address internal and external challenges. ABB Oil, Gas \& Chemical.

Aho, M., Bergman, S., Hammarström, L., Yli-Opas, K., Pelkola, A., Sourander, M., 2009. Closed loop dynamic optimization of a petroleum refinery process. IFAC Proc. Vol. 7, 257-262. https://doi.org/10.3182/20090506-3-SF-4003.00048

Alessandri, A., Baglietto, M., Battistelli, G., Zavala, V., 2010. Advances in moving horizon estimation for nonlinear systems. Proc. IEEE Conf. Decis. Control 5681-5688. https://doi.org/10.1109/CDC.2010.5718126

Allgor, R.J., Barton, P.I., 1999. Mixed-integer dynamic optimization I: Problem formulation. Comput. Chem. Eng. 23, 567-584. https://doi.org/10.1016/S0098-1354(98)00294-4

Allgöwer, F., Badgwell, T.A., Qin, J.S., Rawlings, J.B., Wright, S.J., 1999. Nonlinear Predictive Control and Moving Horizon Estimation - An Introductory Overview BT Advances in Control, in: Frank, P.M. (Ed.), . Springer London, London, pp. 391-449.

Alvarez, L.A., Odloak, D., 2010. Robust integration of real time optimization with linear model predictive control. Comput. Chem. Eng. 34, 1937-1944. https://doi.org/10.1016/j.compchemeng.2010.06.017

Alves, J.J., Towler, G.P., 2002. Analysis of Refinery Hydrogen Distribution Systems. Ind. Eng. Chem. Res. 41, 5759-5769. https://doi.org/10.1021/ie010558v

Amrit, R., Rawlings, J.B., Biegler, L.T., 2013. Optimizing process economics online using model predictive control. Comput. Chem. Eng. 58, 334-343. https://doi.org/https://doi.org/10.1016/j.compchemeng.2013.07.015

ANSI/ISA-95.00.01-2000, 2000. ISA-95.00. 01-2000: Enterprise-control system integration part 1: Models and terminology, Technical report, ISA, The Instrumentation, Systems, and Automation Society.

ANSI/ISA-95.00.03-2005, 2005. Enterprise-Control System Integration. Part 3: Activity models of manufacturing operations management. ISA.

Arora, N., Biegler, L.T., 2001. Redescending estimators for data reconciliation and parameter estimation. Comput. Chem. Eng. 25, 1585-1599. https://doi.org/10.1016/S0098-1354(01)00721-9

Artzner, P., Delbaen, F., Eber, J.-M., Heath, D., 1999. Coherent Measures of Risk. Math. Financ. 9, 203-228. https://doi.org/10.1111/1467-9965.00068

Baldea, M., Harjunkoski, I., 2014. Integrated production scheduling and process control: A 
systematic review. Comput. Chem. Eng. 71, 377-390. https://doi.org/https://doi.org/10.1016/j.compchemeng.2014.09.002

Beck, R., n.d. The Digital Twin and the Smart Enterprise.

Ben-Tal, A., Nemirovski, A., 2002. Robust optimization - methodology and applications. Math. Program. 92, 453-480. https://doi.org/10.1007/s101070100286

Benbasat, I., Nault, B.R., 1990. An evaluation of empirical research in managerial support systems. Decis. Support Syst. 6, 203-226. https://doi.org/10.1016/01679236(90)90015-J

Biegler, L.T., 2018. Advanced optimization strategies for integrated dynamic process operations. Comput. Chem. Eng. 114, 3-13. https://doi.org/10.1016/j.compchemeng.2017.10.016

Biegler, L.T., 2010. Nonlinear Programming, Society for Industrial and Applied Mathematics. Society for Industrial and Applied Mathematics. https://doi.org/10.1137/1.9780898719383

Biegler, L.T., 2000. Efficient Solution of Dynamic Optimization and NMPC Problems BT Nonlinear Model Predictive Control, in: Allgöwer, F., Zheng, A. (Eds.), . Birkhäuser Basel, Basel, pp. 219-243.

Biegler, L.T., Cervantes, A.M., Wächter, A., 2002. Advances in simultaneous strategies for dynamic process optimization. Chem. Eng. Sci. 57, 575-593. https://doi.org/10.1016/S0009-2509(01)00376-1

Biegler, L.T., Thierry, D.M., 2018. Large-scale Optimization Formulations and Strategies for Nonlinear Model Predictive Control. IFAC-PapersOnLine 51, 1-15. https://doi.org/https://doi.org/10.1016/j.ifacol.2018.10.167

Birge, J.R., Louveaux, F., 2011. Introduction to Stochastic Programming, Second. ed, Springer New York Dordrecht Heidelberg London. Springer, New York Dordrecht Heidelberg London. https://doi.org/10.1007/978-1-4614-0237-4

Burnak, B., Diangelakis, N.A., Katz, J., Pistikopoulos, E.N., 2019. Integrated process design, scheduling, and control using multiparametric programming. Comput. Chem. Eng. 125, 164-184. https://doi.org/10.1016/j.compchemeng.2019.03.004

Camacho, E.F., Bordons, C., 2007. Model Predictive control, Studies in Systems, Decision and Control, Advanced Textbooks in Control and Signal Processing. Springer London, London. https://doi.org/10.1007/978-0-85729-398-5

Cameron, I.T., Engell, S., Georgakis, C., Asprion, N., Bonvin, D., Gao, F., Gerogiorgis, D.I., Grossmann, I.E., Macchietto, S., Preisig, H.A., Young, B.R., 2019. Education in Process Systems Engineering: Why it matters more than ever and how it can be structured. 
$\begin{array}{llll}\text { Comput. Chem. } & \text { Eng. 126, }\end{array}$ https://doi.org/10.1016/j.compchemeng.2019.03.036

Campo, P.J., Morari, M., 1987. Robust Model Predictive Control, in: 1987 American Control Conference. pp. 1021-1026. https://doi.org/10.23919/ACC.1987.4789462

Cao, Y., Li, S., Petzold, L., 2002. Adjoint sensitivity analysis for differential-algebraic equations: Algorithms and software. J. Comput. Appl. Math. 149, 171-191. https://doi.org/10.1016/S0377-0427(02)00528-9

Cao, Y., Li, S., Petzold, L., Serban, R., 2003. Adjoint Sensitivity Analysis for DifferentialAlgebraic Equations: The Adjoint DAE System and Its Numerical Solution. SIAM J. Sci. Comput. 24, 1076-1089. https://doi.org/10.1137/s1064827501380630

Chachuat, B., Srinivasan, B., Bonvin, D., 2009. Adaptation strategies for real-time optimization. Comput. Chem. Eng. 33, 1557-1567. https://doi.org/10.1016/j.compchemeng.2009.04.014

Chen, Q., Grossmann, I., 2019. Modern Modeling Paradigms Using Generalized Disjunctive Programming. Processes. https://doi.org/10.3390/pr7110839

Chen, Y., Eslick, J.C., Grossmann, I.E., Miller, D.C., 2015. Simultaneous process optimization and heat integration based on rigorous process simulations. Comput. Chem. Eng. 81, 180-199. https://doi.org/https://doi.org/10.1016/j.compchemeng.2015.04.033

Chen, Y., Lin, M., Jiang, H., Yuan, Z., Chen, B., 2020. Optimal Design and Operation of Refinery Hydrogen Systems under Multi-Scale Uncertainties. Comput. Chem. Eng. 106822. https://doi.org/10.1016/j.compchemeng.2020.106822

Cheng, F.-T., Shen, E., Deng, J.-Y., Nguyen, K., 1999. Development of a system framework for the computer-integrated manufacturing execution system: A distributed objectoriented approach. Int. J. Comput. Integr. Manuf. 12, 384-402. https://doi.org/10.1080/095119299130137

Clarke, D.W., Mohtadi, C., Tuffs, P.S., 1987. Generalized predictive control-Part I. The basic algorithm. Automatica 23, 137-148. https://doi.org/10.1016/00051098(87)90087-2

Cozad, A., Sahinidis, N. V, Miller, D.C., 2014. Learning surrogate models for simulationbased optimization. AIChE J. 60, 2211-2227. https://doi.org/10.1002/aic.14418

Cutler, C.R. (Charles R., 1983. Dynamic matrix control : an optimal multivariable control algorithm with constraints. Thesis (Ph. D.)--University of Houston-University Park, 1983.

Cutler, C.R., Ramaker, L.B., 1979. Dynamic matrix control- a computer control algorithm, in: AIChE 86th Spring Meeting,. Houston, Texas, p. Paper No. 51b. 
Daoutidis, P., Lee, J.H., Harjunkoski, I., Skogestad, S., Baldea, M., Georgakis, C., 2018 a. Integrating operations and control: A perspective and roadmap for future research. Comput. Chem. Eng. 115, 179-184. https://doi.org/https://doi.org/10.1016/j.compchemeng.2018.04.011

Daoutidis, P., Tang, W., Jogwar, S.S., 2018b. Decomposing complex plants for distributed control: Perspectives from network theory. Comput. Chem. Eng. 114, 43-51. https://doi.org/https://doi.org/10.1016/j.compchemeng.2017.10.015

Darby, M.L., Nikolaou, M., Jones, J., Nicholson, D., 2011. RTO: An overview and assessment of current practice. J. Process Control 21, 874-884. https://doi.org/https://doi.org/10.1016/j.jprocont.2011.03.009

De Keyser, R.M.C., Van Cauwenberghe, A.R., 1985. Extended Prediction Self-Adaptive Control. IFAC Proc. Vol. 18, 1255-1260. https://doi.org/10.1016/S1474$6670(17) 60736-5$

De Keyser, R.M.C., Van Cauwenberghe, A.R., 1981. A self-tuning multistep predictor application. Automatica 17, 167-174. https://doi.org/10.1016/0005-1098(81)900923

de Prada, C., Pitarch, J.L., 2018. Real-Time Optimization (RTO) Systems, in: Resource Efficiency of Processing Plants, Wiley Online Books. Wiley-VCH Verlag GmbH \& Co. KGaA, Weinheim, Germany, pp. 265-292. https://doi.org/10.1002/9783527804153.ch11

de Prada, C., Sarabia, D., 2018. Data Pre-treatment, in: Resource Efficiency of Processing Plants. Wiley-VCH Verlag GmbH \& Co. KGaA, Weinheim, Germany, pp. 181-210. https://doi.org/10.1002/9783527804153.ch8

de Prada, C., Sarabia, D., Gutierrez, G., Gomez, E., Marmol, S., Sola, M., Pascual, C., Gonzalez, R., 2017. Integration of RTO and MPC in the Hydrogen Network of a Petrol Refinery. Processes 5, 3. https://doi.org/10.3390/pr5010003

de Prada, C., Valentín, A., 1996. Set Point Optimization in Multivariable Constrained Predictive Control. IFAC Proc. Vol. 29, 1792-1797. https://doi.org/10.1016/S14746670(17)57929-X

De Souza, G., Odloak, D., Zanin, A.C., 2010. Real time optimization (RTO) with model predictive control (MPC). Comput. Chem. Eng. 34, 1999-2006. https://doi.org/10.1016/j.compchemeng.2010.07.001

Dias, L.S., lerapetritou, M.G., 2016. Integration of scheduling and control under uncertainties: Review and challenges. Chem. Eng. Res. Des. 116, 98-113. https://doi.org/https://doi.org/10.1016/j.cherd.2016.10.047

Doherty, M.F., Grossmann, I.E., Pantelides, C.C., 2016. A tribute to professor Roger 
Sargent: Intellectual leader of process systems engineering. AIChE J. 62, 2951-2958. https://doi.org/10.1002/aic.15425

Drud, A., 1985. CONOPT: A GRG code for large sparse dynamic nonlinear optimization problems. Math. Program. 31, 153-191. https://doi.org/10.1007/BF02591747

Drud, A.S., 2002. On the Use of Second Order Information in GAMS/CONOPT3. SIAM Optim. Meet.

Drud, A.S., 1994. CONOPT-A Large-Scale GRG Code. ORSA J. Comput. 6, 207-216. https://doi.org/10.1287/ijoc.6.2.207

EA Internacional, 2019a. PROOSIS.

EA Internacional, 2019b. PROOSIS2019. Complete Reference Manual. Madrid, Spain.

Ellis, M., Christofides, P.D., 2015. Real-time economic model predictive control of nonlinear process systems. AIChE J. 61, 555-571. https://doi.org/10.1002/aic.14673

Ellis, M., Durand, H., Christofides, P.D., 2014. A tutorial review of economic model predictive control methods. J. Process Control 24, 1156-1178. https://doi.org/10.1016/j.jprocont.2014.03.010

Elsherif, M., Manan, Z.A., Kamsah, M.Z., 2015. State-of-the-art of hydrogen management in refinery and industrial process plants. J. Nat. Gas Sci. Eng. 24, 346-356. https://doi.org/10.1016/j.jngse.2015.03.046

Engell, S., 2007. Feedback control for optimal process operation. J. Process Control 17, 203-219. https://doi.org/10.1016/j.jprocont.2006.10.011

Engell, S., Harjunkoski, I., 2012. Optimal operation: Scheduling, advanced control and their integration. Comput. Chem. Eng. 47, 121-133. https://doi.org/10.1016/j.compchemeng.2012.06.039

Erkoyuncu, J., Birkin, G., levy Carvalho do lago, R., Williamson, A., Williams, H., Roy, R., n.d. Conceptualisation of digital twins in the through-life engineering services environment. Unpublished book chapter.

F-Chart.Software, 2020. EES: Engineering Equation Solver.

Forbes, J.F., Marlin, T.E., 1994. Model Accuracy for Economic Optimizing Controllers: The Bias Update Case. Ind. Eng. Chem. Res. 33, 1919-1929. https://doi.org/10.1021/ie00032a006

Forbes, J.F., Marlin, T.E., MacGregor, J.F., 1992. Model Accuracy Requirments For Economic Optimizing Model Predictive Controllers - The Linear Programming Case, in: 1992 American Control Conference. pp. 1587-1593. 
https://doi.org/10.23919/ACC.1992.4792375

Fraedrich, D., Goldberg, A., 2000. Methodological framework for the validation of predictive simulations. Eur. J. Oper. Res. 124, 55-62. https://doi.org/10.1016/S03772217(99)00117-4

Fritzson, P., 2014. Cyber-Physical System Modeling Methodology, in: Principles of Object Oriented Modeling and Simulation with Modelica 3.3, Wiley Online Books. pp. 565589. https://doi.org/10.1002/9781118989166.ch12

Fritzson, P., 2009. Principles of Modeling and Simulation, Principles of Modeling and Simulation. John Wiley \& Sons, Inc., Hoboken, NJ, USA. https://doi.org/10.1002/9780470403563

Froment, G.F., 2004. Modeling in the development of hydrotreatment processes. Catal. Today 98, 43-54. https://doi.org/10.1016/j.cattod.2004.07.052

Galan, A., De Prada, C., Gutierrez, G., Sarabia, D., Gonzalez, R., 2019a. Predictive Simulation Applied to Refinery Hydrogen Networks for Operators' Decision Support. IFAC-PapersOnLine 52, 862-867. https://doi.org/10.1016/j.ifacol.2019.06.170

Galan, A., de Prada, C., Gutierrez, G., Sarabia, D., Gonzalez, R., Sola, M., Marmol, S., 2018. Validation of a hydrogen network RTO application for decision support of refinery $\begin{array}{lll}\text { operators. } & \text { IFAC-PapersOnLine 73-78. }\end{array}$ https://doi.org/https://doi.org/10.1016/j.ifacol.2018.09.256

Galan, A., de Prada, C., Gutierrez, G., Sarabia, D., Gonzalez, R., Sola, M., Marmol, S., Pascual, C., 2017. Resource efficiency indicators usefulness for decision-making process of operators: refinery hydrogen network case study, in: Espuña, A., Graells, M., Puigjaner, L.B.T.-C.A.C.E. (Eds.), 27 European Symposium on Computer Aided Process Engineering. Elsevier, pp. 1513-1518. https://doi.org/10.1016/B978-0-44463965-3.50254-3

Galan, A., de Prada, C., Gutierrez, G., Sarabia, D., Grossmann, I.E., Gonzalez, R., $2019 b$. Implementation of RTO in a large hydrogen network considering uncertainty. Optim. Eng. https://doi.org/10.1007/s11081-019-09444-3

GAMS Development Corporation, 2019. General Algebraic Modeling System (GAMS) Release 27.1.0.

Gao, W., Engell, S., 2005. Iterative set-point optimization of batch chromatography. $\begin{array}{llll}\text { Comput. Chem. } \quad \text { Eng. 1401-1409. } & \end{array}$ https://doi.org/10.1016/j.compchemeng.2005.02.035

García, C.E., Prett, D.M., Morari, M., Garcia, C.E., Prett, D.M., 1989. Model predictive control: Theory and practice-A survey. Automatica 25, 335-348. https://doi.org/https://doi.org/10.1016/0005-1098(89)90002-2 
Gill, P., Murray, W., Saunders, M., 2005. SNOPT: An SQP Algorithm for Large-Scale Constrained Optimization. SIAM Rev. 47, 99-131. https://doi.org/10.1137/S0036144504446096

Gill, P.E., Murray, W., Saunders, M.A., Wong, E., 2018. User's Guide for \{SNOPT 7.7\}: Software for Large-Scale Nonlinear Programming. La Jolla, CA.

Gillette, R.D., Prett, D.M., 1979. Optimization and constrained multivariable control of a catalytic cracking unit, in: AIChE 86th Annual Meeting. p. Paper No. 51c.

Girardin, L., Marechal, F., Tromeur, P., 2006. Methodology for the design of industrial hydrogen networks and the optimal placement of purification units using multiobjective optimisation techniques, in: Marquardt, W., Pantelides, C.B.T.-C.A.C.E. (Eds.), 16th European Symposium on Computer Aided Process Engineering and 9th International Symposium on Process Systems Engineering. Elsevier, pp. 1765-1770. https://doi.org/https://doi.org/10.1016/S1570-7946(06)80303-2

Goel, V., Grossmann, I.E., 2004. A stochastic programming approach to planning of offshore gas field developments under uncertainty in reserves. Comput. Chem. Eng. 28, 1409-1429. https://doi.org/10.1016/j.compchemeng.2003.10.005

Gómez Sayalero, E.M., 2016. A study on modelling, data reconciliation and optimal operation of hydrogen networks in oil refineries. Universidad de Valladolid. https://doi.org/10.35376/10324/16637

González-Martín, R., Morilla, F., 2019. Optimización y control de un proceso de mezclas Rundown para la fabricación de gasolinas. Rev. Iberoam. Automática e Informática Ind. Vol. 16, N. https://doi.org/10.4995/riai.2019.10639

Gonzalez Santos, A.I., Zamarreno Cosme, J.M., de Prada Moraga, C., 2001. Nonlinear model predictive control in a batch fermentator with state estimation, in: 2001 European Control Conference (ECC). IEEE, pp. 426-431. https://doi.org/10.23919/ECC.2001.7075944

Goux, J.-P., Leyffer, S., 2002. Solving Large MINLPs on Computational Grids. Optim. Eng. 3, 327-346. https://doi.org/10.1023/A:1021047328089

Grossmann, I., 2005. Enterprise-wide optimization: A new frontier in process systems engineering. AIChE J. 51, 1846-1857. https://doi.org/10.1002/aic.10617

Grossmann, I.E., 2014. Challenges in the application of mathematical programming in the enterprise-wide optimization of process industries. Theor. Found. Chem. Eng. 48, 555-573. https://doi.org/10.1134/S0040579514050182

Grossmann, I.E., 2012. Advances in mathematical programming models for enterprisewide optimization. Comput. Chem. Eng. 47, 2-18. https://doi.org/https://doi.org/10.1016/j.compchemeng.2012.06.038 
Grossmann, I.E., Apap, R.M., Calfa, B.A., García-Herreros, P., Zhang, Q., 2016. Recent advances in mathematical programming techniques for the optimization of process systems under uncertainty. Comput. Chem. Eng. 91, 3-14. https://doi.org/https://doi.org/10.1016/j.compchemeng.2016.03.002

Grossmann, I.E., Biegler, L.T., 1995. Optimizing Chemical Processes. Chemtech 27-35.

Grossmann, Ignacio E., Harjunkoski, I., 2019. Process Systems Engineering: Academic and Industrial Perspectives. Comput. Chem. Eng. 126, 474-484. https://doi.org/10.1016/J.COMPCHEMENG.2019.04.028

Grossmann, Ignacio E, Harjunkoski, I., 2019. Process Systems Engineering: Academic and industrial perspectives. Comput. Chem. Eng. 126, 474-484. https://doi.org/10.1016/j.compchemeng.2019.04.028

Grossmann, I.E., McDonald, C.M., 2004. Foreword. Comput. Chem. Eng. 28, 853. https://doi.org/https://doi.org/10.1016/j.compchemeng.2003.09.012

Gupta, V., Grossmann, I.E., 2011. Solution strategies for multistage stochastic programming with endogenous uncertainties. Comput. Chem. Eng. 35, 2235-2247. https://doi.org/10.1016/j.compchemeng.2010.11.013

Gutierrez, G., Galan, A., Sarabia, D., de Prada, C., 2018. Two-Stage Stochastic Optimization of a hydrogen network. IFAC-PapersOnLine 51, 263-268. https://doi.org/https://doi.org/10.1016/j.ifacol.2018.09.310

Haag, S., Anderl, R., 2018. Digital twin - Proof of concept. Manuf. Lett. 15, 64-66. https://doi.org/10.1016/j.mfglet.2018.02.006

Halim, I., Srinivasan, R., 2011. A knowledge-based simulation-optimization framework and system for sustainable process operations. Comput. Chem. Eng. 35, 92-105. https://doi.org/https://doi.org/10.1016/j.compchemeng.2010.08.004

Harjunkoski, I., 2016. Deploying scheduling solutions in an industrial environment. Comput. Chem. Eng. 91, 127-135. https://doi.org/10.1016/j.compchemeng.2016.03.029

Harjunkoski, I., Maravelias, C.T., Bongers, P., Castro, P.M., Engell, S., Grossmann, I.E., Hooker, J., Méndez, C., Sand, G., Wassick, J., 2014. Scope for industrial applications of production scheduling models and solution methods. Comput. Chem. Eng. 62, 161-193. https://doi.org/https://doi.org/10.1016/j.compchemeng.2013.12.001

Harjunkoski, I., Nyström, R., Horch, A., 2009. Integration of scheduling and controlTheory or practice? Comput. Chem. Eng. 33, 1909-1918. https://doi.org/10.1016/j.compchemeng.2009.06.016

Hedengren, J.D., Eaton, A.N., 2017. Overview of estimation methods for industrial 
dynamic systems. Optim. Eng. 18, 155-178. https://doi.org/10.1007/s11081-0159295-9

Heinzl, B., Raich, P., Preyser, F., Kastner, W., 2018. Simulation-based Assessment of Energy Efficiency in Industry: Comparison of Hybrid Simulation Approaches. IFACPapersOnLine 51 ,

689-694. https://doi.org/https://doi.org/10.1016/j.ifacol.2018.03.117

Heirung, T.A.N., Paulson, J.A., O'Leary, J., Mesbah, A., 2018. Stochastic model predictive control - how does it work? Comput. Chem. Eng. 114, 158-170. https://doi.org/10.1016/j.compchemeng.2017.10.026

Ho, T.C., 2004. Deep HDS of diesel fuel: chemistry and catalysis. Catal. Today 98, 3-18. https://doi.org/10.1016/j.cattod.2004.07.048

Huber, P.J., 2011. Robust Statistics, in: Lovric, M. (Ed.), International Encyclopedia of Statistical Science. Springer Berlin Heidelberg, Berlin, Heidelberg, pp. 1248-1251. https://doi.org/10.1007/978-3-642-04898-2

IEA, 2019. World Energy Outlook 2019. Paris.

Ishii, Y., Otto, F.D., 2018. Decoupling the constraints for process simulation in large-scale flowsheet optimization. Comput. Chem. Eng. 113, 240-252. https://doi.org/10.1016/j.compchemeng.2018.03.018

Jia, N., Zhang, N., 2011. Multi-component optimisation for refinery hydrogen networks. Energy 36, 4663-4670. https://doi.org/10.1016/j.energy.2011.03.040

Jiao, Y., Su, H., Hou, W., 2012a. Improved optimization methods for refinery hydrogen network and their applications. Control Eng. Pract. 20, 1075-1093. https://doi.org/https://doi.org/10.1016/j.conengprac.2012.04.010

Jiao, Y., Su, H., Hou, W., Liao, Z., 2012b. Optimization of refinery hydrogen network based on chance constrained programming. Chem. Eng. Res. Des. 90, 1553-1567. https://doi.org/10.1016/j.cherd.2012.02.016

KBC, 2018. Digitalization Manifesto [WWW Document]. URL https://www.kbc.global/insights/whitepapers/digitalization-manifesto (accessed 6.6.19).

Kondili, E., Pantelides, C.C., Sargent, R.W.H., 1993. A general algorithm for short-term scheduling of batch operations-I. MILP formulation. Comput. Chem. Eng. 17, 211227. https://doi.org/https://doi.org/10.1016/0098-1354(93)80015-F

Kouyionas, V., 1990. INTERACTIONS OF COMPETING CATALYTIC HYDROTREATING REACTIONS. Imperial College of London. 
Krämer, S., Engell, S., 2018. Energy and Resource Efficiency in the Process Industries, in: Resource Efficiency of Processing Plants, Wiley Online Books. Wiley-VCH Verlag $\mathrm{GmbH} \&$ Co. KGaA, Weinheim, Germany, pp. 3-18. https://doi.org/10.1002/9783527804153.ch1

Krishnamoorthy, D., Foss, B., Skogestad, S., 2018. Steady-state real-time optimization using transient measurements. Comput. Chem. Eng. 115, 34-45. https://doi.org/https://doi.org/10.1016/j.compchemeng.2018.03.021

Kujanpää, M., Hakala, J., Pajula, T., Beisheim, B., Krämer, S., Ackerschott, D., Kalliski, M., Engell, S., Enste, U., Pitarch Perez, J.L., 2017. Successful Resource Efficiency Indicators for process industries, Step-by-step guidebook, VTT Technology BT Successful Resource Efficiency Indicators for process industries. VTT Technical Research Centre of Finland, Espoo.

Kumar, A., Gautami, G., Khanam, S., 2010. Hydrogen distribution in the refinery using mathematical modeling. Energy 35, 3763-3772. https://doi.org/10.1016/j.energy.2010.05.025

Lee, J.H., 2011. Model predictive control: Review of the three decades of development. Int. J. Control. Autom. Syst. 9, 415. https://doi.org/10.1007/s12555-011-0300-6

Li, D., 2016. Perspective for smart factory in petrochemical industry. Comput. Chem. Eng. 91, 136-148. https://doi.org/10.1016/j.compchemeng.2016.03.006

Li, X., Chen, Y., Barton, P.I., 2012. Nonconvex Generalized Benders Decomposition with Piecewise Convex Relaxations for Global Optimization of Integrated Process Design and Operation Problems. Ind. Eng. Chem. Res. 51, 7287-7299. https://doi.org/10.1021/ie201262f

Li, X., Tomasgard, A., Barton, P.I., 2017. Natural gas production network infrastructure development under uncertainty. Optim. Eng. 18, 35-62. https://doi.org/10.1007/s11081-016-9322-5

Linderoth, J., Wright, S., 2003. Decomposition Algorithms for Stochastic Programming on a Computational Grid. Comput. Optim. Appl. 24, 207-250. https://doi.org/10.1023/A:1021858008222

Lou, J., Liao, Z., Jiang, B., Wang, J., Yang, Y., 2014. Robust optimization of hydrogen network. Int. J. Hydrogen Energy 39, 1210-1219. https://doi.org/https://doi.org/10.1016/j.ijhydene.2013.11.024

Lucia, S., Engell, S., 2015. Potential and limitations of multi-stage nonlinear model predictive control. IFAC-PapersOnLine 28, 1015-1020. https://doi.org/10.1016/j.ifacol.2015.09.101

Lucia, S., Finkler, T., Engell, S., 2013. Multi-stage nonlinear model predictive control 
applied to a semi-batch polymerization reactor under uncertainty. J. Process Control 23, 1306-1319. https://doi.org/10.1016/j.jprocont.2013.08.008

Lundström, P., Lee, J.H., Morari, M., Skogestad, S., 1995. Limitations of dynamic matrix control. Comput. Chem. Eng. 19, 409-421. https://doi.org/https://doi.org/10.1016/0098-1354(94)00063-T

MathWorks, 2020. Simulation and Model-Based Design.

Mayne, D.Q., Rawlings, J.B., Rao, C.V., Scokaert, P.O.M., 2000. Constrained model predictive control: Stability and optimality. Automatica 36, 789-814. https://doi.org/10.1016/S0005-1098(99)00214-9

Méndez, C.A., Cerdá, J., Grossmann, I.E., Harjunkoski, I., Fahl, M., 2006. State-of-the-art review of optimization methods for short-term scheduling of batch processes. Comput. Chem. Eng. 30, 913-946. https://doi.org/https://doi.org/10.1016/j.compchemeng.2006.02.008

Misener, R., Thompson, J.P., Floudas, C.A., 2011. APOGEE: Global optimization of standard, generalized, and extended pooling problems via linear and logarithmic partitioning schemes. Comput. Chem. Eng. 35, 876-892. https://doi.org/10.1016/j.compchemeng.2011.01.026

Morari, M., Arkun, Y., Stephanopoulos, G., 1980. Studies in the synthesis of control structures for chemical processes: Part I: Formulation of the problem. Process decomposition and the classification of the control tasks. Analysis of the optimizing control structures. AIChE J. 26, 220-232. https://doi.org/10.1002/aic.690260205

Morari, M., Lee, J.H., 2014. Model Predictive Control: Past, Present and Future. Neuroscience 23, 667-682. https://doi.org/10.1016/j.neuroscience.2014.03.010

Morari, M., Stephanopoulos, G., 1980. Studies in the synthesis of control structures for chemical processes: Part II: Structural aspects and the synthesis of alternative feasible control schemes. AIChE J. 26, 232-246. https://doi.org/10.1002/aic.690260206

Mouret, S., Grossmann, I.E., Pestiaux, P., 2009. A Novel Priority-Slot Based ContinuousTime Formulation for Crude-Oil Scheduling Problems. Ind. Eng. Chem. Res. 48, 85158528. https://doi.org/10.1021/ie8019592

Mulvey, J.M., Vanderbei, R.J., Zenios, S.A., 1995. Robust Optimization of Large-Scale Systems. Oper. Res. 43, 264-281. https://doi.org/10.1287/opre.43.2.264

Muñoz, E., Capón-García, E., Moreno-Benito, M., Espuña, A., Puigjaner, L., 2011. Scheduling and control decision-making under an integrated information environment. Comput. Chem. Eng. 35, 774-786. https://doi.org/10.1016/j.compchemeng.2011.01.025 
NAMUR, 2020. NAMUR Open Architecture Overview NOA.

NAMUR, 2018. Association of Automation Technology in Process Industries [WWW Document]. URL https://www.namur.net/en/index.html (accessed 6.1.20).

Navarro, A.K.W., Vassiliadis, V.S., 2014. Computer algebra systems coming of age: Dynamic simulation and optimization of DAE systems in Mathematica ${ }^{\mathrm{TM}}$. Comput. $\begin{array}{lll}\text { Chem. } & \text { Eng. 62, 125-138. }\end{array}$ https://doi.org/https://doi.org/10.1016/j.compchemeng.2013.11.004

Navia, D., Sarabia, D., Gutiérrez, G., Cubillos, F., de Prada, C., 2014. A comparison between two methods of stochastic optimization for a dynamic hydrogen consuming plant. Comput. Chem. Eng. 63, 219-233. https://doi.org/10.1016/j.compchemeng.2014.02.004

Nicholson, B., López-Negrete, R., Biegler, L.T., 2014. On-line state estimation of nonlinear dynamic systems with gross errors. Comput. Chem. Eng. 70, 149-159. https://doi.org/10.1016/j.compchemeng.2013.11.018

OPAF, 2019. Open Process Automation Forum: Brochure.

OPAF, 2018. Open Process Automation ${ }^{\mathrm{TM}}$ Forum [WWW Document]. URL https://www.opengroup.org/forum/open-process-automation-forum (accessed 6.1.20).

Özyurt, D.B., Pike, R.W., 2004. Theory and practice of simultaneous data reconciliation and gross error detection for chemical processes. Comput. Chem. Eng. 28, 381-402. https://doi.org/10.1016/j.compchemeng.2003.07.001

Pablos, C., Merino, A., Acebes, L.F., 2019. Modeling On-Site Combined Heat and Power Systems Coupled to Main Process Operation. Processes 7, 218. https://doi.org/10.3390/pr7040218

Pantelides, C.C., 1994. Unified frameworks for optimal process planning and scheduling, in: Proceedings on the Second Conference on Foundations of Computer Aided Operations. pp. 253-274.

Papageorgiou, L.G., 2009. Supply chain optimisation for the process industries: Advances and opportunities. Comput. Chem. Eng. 33, 1931-1938. https://doi.org/10.1016/j.compchemeng.2009.06.014

Perea-López, E., Ydstie, B.E., Grossmann, I.E., 2003. A model predictive control strategy for supply chain optimization. Comput. Chem. Eng. 27, 1201-1218. https://doi.org/10.1016/S0098-1354(03)00047-4

Perino, J., 2019. The Process Manufacturing Digital Twin: Digitalization of the Asset Lifecycle and Value Chain. 
Petronor, 2020. Petronor [WWW Document]. URL https://petronor.eus/es/ (accessed 6.1.20).

Pflug, G.C., 2000. Some Remarks on the Value-at-Risk and the Conditional Value-at-Risk. pp. 272-281. https://doi.org/10.1007/978-1-4757-3150-7_15

Pitarch, L.J., Sala, A., de Prada, C., 2019. A Systematic Grey-Box Modeling Methodology via Data Reconciliation and SOS Constrained Regression. Processes. https://doi.org/10.3390/pr7030170

Prades, L., Romero, F., Estruch, A., García-Dominguez, A., Serrano, J., 2013. Defining a Methodology to Design and Implement Business Process Models in BPMN According to the Standard ANSI/ISA-95 in a Manufacturing Enterprise. Procedia Eng. 63, 115122. https://doi.org/https://doi.org/10.1016/j.proeng.2013.08.283

Rao, C. V, Rawlings, J.B., 2000. Nonlinear Moving Horizon State Estimation BT - Nonlinear Model Predictive Control, in: Allgöwer, F., Zheng, A. (Eds.), . Birkhäuser Basel, Basel, pp. 45-69.

Rawlings, J.B., 2009. State Estimation using Moving Horizon Estimation and Particle Filtering. UW Math Probab. Semin. 1-45.

Richalet, J., Rault, A., Testud, J.L., Papon, J., 1978. Model predictive heuristic control. Automatica 14, 413-428. https://doi.org/10.1016/0005-1098(78)90001-8

ROBERTS, P.D., 1979. An algorithm for steady-state system optimization and parameter $\begin{array}{llllll}\text { estimation. Int. Syst. J. Sci. } & & \text { 10, }\end{array}$ https://doi.org/10.1080/00207727908941614

Rockafellar, R.T., Uryasev, S., 2002. Conditional value-at-risk for general loss distributions. J. Bank. Financ. 26, 1443-1471. https://doi.org/10.1016/S0378-4266(02)00271-6

Rockafellar, R.T., Uryasev, S., 2000. Optimization of Conditional Value-at-Risk. J. Risk 2, 2141.

Rodríguez-Blanco, T., 2017. Modifier Adaptation for process optimization with uncertainty. Universidad de Valladolid.

Rodríguez-Blanco, T., Sarabia, D., de Prada, C., 2019. Efficient nested modifier-adaptation methodology for dealing with process constraints in real-time optimization. Comput. Chem. Eng. 122, 372-382. https://doi.org/10.1016/j.compchemeng.2018.08.015

Rodriguez-Blanco, T., Sarabia, D., Navia, D., De Prada, C., 2015. Modifier-Adaptation methodology for RTO applied to Distillation Columns. IFAC-PapersOnLine 48, 223228. https://doi.org/10.1016/j.ifacol.2015.08.185

Rolandi, P.A., Romagnoli, J.A., 2010. Integrated model-centric framework for support of 
manufacturing operations. Part I: The framework. Comput. Chem. Eng. 34, 17-35. https://doi.org/https://doi.org/10.1016/j.compchemeng.2009.08.006

Saenz de Ugarte, B., Artiba, A., Pellerin, R., 2009. Manufacturing execution system - a literature review. Prod. Plan. Control 20, 525-539. https://doi.org/10.1080/09537280902938613

Sahinidis, N. V., 2004. Optimization under uncertainty: State-of-the-art and opportunities. Comput. Chem. Eng. 28, 971-983. https://doi.org/10.1016/j.compchemeng.2003.09.017

Sarabia, D., de Prada, C., Gómez, E., Gutierrez, G., Cristea, S., Sola, J.M., Gonzalez, R., 2012. Data reconciliation and optimal management of hydrogen networks in a petrol refinery. Control Eng. Pract. 20, 343-354. https://doi.org/10.1016/j.conengprac.2011.06.009

Sardashti Birjandi, M.R., Shahraki, F., Birjandi, M.S., Nobandegani, M.S., 2014. Application of global optimization strategies to refinery hydrogen network. Int. J. Hydrogen Energy 39, 14503-14511. https://doi.org/10.1016/j.ijhydene.2014.07.047

Sargent, R., 1967. Integrated Design and Optimization of Processes. Chem. Eng. Prog. 63, 71-78.

Schweiger, G., Nilsson, H., Schoeggl, J., Birk, W., Posch, A., 2020. Modeling and simulation of large-scale systems: A systematic comparison of modeling paradigms. Appl. Math. Comput. 365, 124713. https://doi.org/https://doi.org/10.1016/j.amc.2019.124713

Serban, R., Petra, C., Hindmarsh, A.C., 2019. User Documentation for idas v3.1.0 ( sundials v4.1.0) [WWW Document]. URL https://computation.Ilnl.gov/sites/default/files/public/idas_guide.pdf (accessed 6.18.19).

Shapiro, J.F., 2004. Challenges of strategic supply chain planning and modeling. Comput. Chem. Eng. 28, 855-861. https://doi.org/10.1016/j.compchemeng.2003.09.013

Shariati, M., Tahouni, N., Panjeshahi, M.H., 2013. Investigation of different approaches for hydrogen management in petrochemical complexes. Int. J. Hydrogen Energy 38, 3257-3267. https://doi.org/10.1016/j.ijhydene.2012.12.063

Shobrys, D.E., White, D.C., 2002. Planning, scheduling and control systems: why cannot they work together. Comput. Chem. Eng. 26, 149-160. https://doi.org/https://doi.org/10.1016/S0098-1354(01)00737-2

Skogestad, S., 2000. Plantwide control: the search for the self-optimizing control structure. J. Process Control 10, 487-507. https://doi.org/https://doi.org/10.1016/S09591524(00)00023-8 
Sprague, R.H., 1980. A Framework for the Development of Decision Support Systems. MIS Q. 4, 1. https://doi.org/10.2307/248957

Srinivasan, B., 2007. Real-time optimization of dynamic systems using multiple units. Int. J. Robust Nonlinear Control 17, 1183-1193. https://doi.org/10.1002/rnc.1165

Stark, R., Fresemann, C., Lindow, K., 2019. Development and operation of Digital Twins for technical systems and Services. Ann. https://doi.org/https://doi.org/10.1016/j.cirp.2019.04.024

Stechlinski, P., Patrascu, M., Barton, P.I., 2018. Nonsmooth differential-algebraic equations in chemical engineering. Comput. Chem. Eng. 114, 52-68. https://doi.org/https://doi.org/10.1016/j.compchemeng.2017.10.031

Stephanopoulos, G., Ng, C., 2000. Perspectives on the synthesis of plant-wide control structures. J. Process Control 10, 97-111. https://doi.org/10.1016/S09591524(99)00023-2

Suvarna, M., Divakaran, M., Nduagu, E.I., 2019. Process modelling and simulation of bitumen mining and recovery from oil sands. Miner. Eng. 134, 65-76. https://doi.org/https://doi.org/10.1016/j.mineng.2018.12.024

Tatjewski, P., Tadej, W., 2001. Analysis of an Isope-Type Dual Algorithm for Optimizing Control and Nonlinear Optimization. Int. J. Appl. Math. Comput. Sci. 11, 429-457.

Terrazas-Moreno, S., Grossmann, I.E., Wassick, J.M., Bury, S.J., 2010. Optimal design of reliable integrated chemical production sites. Comput. Chem. Eng. 34, 1919-1936. https://doi.org/10.1016/j.compchemeng.2010.07.027

Thierry, D., Nicholson, B., Biegler, L., 2018. A General Framework for Sensitivity-Based Optimal Control and State Estimation, in: Eden, M.R., lerapetritou, M.G., Towler, G.P.B.T.-C.A.C.E. (Eds.), 13 International Symposium on Process Systems Engineering (PSE 2018). Elsevier, pp. 787-792. https://doi.org/10.1016/B978-0-444-642417.50126-9

Towler, G.P., Mann, R., Serriere, A.J.-L., Gabaude, C.M.D., 1996. Refinery Hydrogen Management: Cost Analysis of Chemically-Integrated Facilities. Ind. Eng. Chem. Res. 35, 2378-2388. https://doi.org/10.1021/ie950359+

Tran, M., Varvarezos, D.K., Nasir, M., 2005. The importance of first-principles, modelbased steady-state gain calculations in model predictive control - A refinery case study. Control Eng. Pract. 13, 1369-1382. https://doi.org/10.1016/j.conengprac.2004.11.015

Trespalacios, F., Grossmann, I.E., 2014. Review of Mixed-Integer Nonlinear and Generalized Disjunctive Programming Methods. Chemie Ing. Tech. 86, 991-1012. https://doi.org/10.1002/cite.201400037 
TRNSYS, 2020. Transient System Simulation Tool.

Umana, B., Shoaib, A., Zhang, N., Smith, R., 2014. Integrating hydroprocessors in refinery hydrogen network optimisation. Appl. Energy 133, 169-182. https://doi.org/https://doi.org/10.1016/j.apenergy.2014.06.080

UNFCCC, 2015. The Paris Agreement [WWW Document]. URL https://unfccc.int/files/essential_background/convention/application/pdf/english_p aris_agreement.pdf (accessed 5.8.20).

Uryasev, S., Rockafellar, R.T., 2001. Conditional Value-at-Risk: Optimization Approach. pp. 411-435. https://doi.org/10.1007/978-1-4757-6594-6_17

Vázquez, F., Jiménez, J., Garrido, J., Belmonte, A., 2010. Introduction to Modelling and Simulation with EcosimPro, Fuera de colección Out of series. Pearson Educación, Madrid, Spain.

Vrabič, R., Erkoyuncu, J.A., Butala, P., Roy, R., 2018. Digital twins: Understanding the added value of integrated models for through-life engineering services. Procedia Manuf. 16, 139-146. https://doi.org/https://doi.org/10.1016/j.promfg.2018.10.167

Wächter, A., Biegler, L.T., 2006. On the implementation of an interior-point filter linesearch algorithm for large-scale nonlinear programming. Math. Program. 106, 25-57. https://doi.org/10.1007/s10107-004-0559-y

Wassick, J.M., 2009. Enterprise-wide optimization in an integrated chemical complex. Comput. Chem. Eng. 33, 1950-1963. https://doi.org/https://doi.org/10.1016/j.compchemeng.2009.06.002

Yang, M., Feng, X., 2019. Simulation-based optimization and design of refinery hydrogen networks with hydrogen sulfide removal. Int. J. Hydrogen Energy. https://doi.org/10.1016/j.ijhydene.2019.07.108

You, F., Grossmann, I.E., 2013. Multicut Benders decomposition algorithm for process supply chain planning under uncertainty. Ann. Oper. Res. 210, 191-211. https://doi.org/10.1007/s10479-011-0974-4

You, F., Wassick, J.M., Grossmann, I.E., 2009. Risk management for a global supply chain planning under uncertainty: Models and algorithms. AIChE J. 55, 931-946. https://doi.org/10.1002/aic.11721

Young, R.E., Bartusiak, R.D., Fontaine, R.W., 2002. Evolution of an Industrial Nonlinear Model Predictive Controller, in: Proc. of Chem Process Control VI. pp. 342-351.

Zavala, V.M., Biegler, L.T., 2009. The advanced-step NMPC controller: Optimality, stability and robustness. Automatica 86-93. https://doi.org/10.1016/j.automatica.2008.06.011 
Zavala, V.M., Laird, C.D., Biegler, L.T., 2008. A fast moving horizon estimation algorithm based on nonlinear programming sensitivity. J. Process Control 18, 876-884. https://doi.org/10.1016/j.jprocont.2008.06.003

Zhang, Q., Grossmann, I.E., 2016. Enterprise-wide optimization for industrial demand side management: Fundamentals, advances, and perspectives. Chem. Eng. Res. Des. 116, 114-131. https://doi.org/https://doi.org/10.1016/j.cherd.2016.10.006

Zimmermann, H.-J., 2001. Fuzzy Set Theory-and Its Applications. Springer Netherlands, Dordrecht. https://doi.org/10.1007/978-94-010-0646-0 

APPENDIXES 



\section{APPENDIX A}

\section{A.1 Simulation AND OPTIMIZATION FOUNDATIONS}

Optimization and simulation are both central to systematic decision making, as well as core subjects in process systems engineering. In general, simulation is useful for its forecasting capability given a model and initial conditions. Similarly, optimization is regarded for its capability for providing the optimal decisions given an objective function and restrictions. Therefore, simulation and optimization both can contribute in decisionmaking processes, whereas from different roles. Actually, mathematical models underpin both simulation and optimization families of techniques, which ultimately explains the fundamentals of their natural interaction. For the sake of understanding I provide a succinct description of simulation and optimization fundamentals, which are developed further into reviews in subsequent sections of the introduction.

\section{A.1.1 SimULATION FUNDAMENTALS}

Firstly, it is important to properly define simulation as an experiment conducted on a model. Although the definition is general, the reference in this thesis is to mathematical models unless stated otherwise. Additionally, it should be pointed out that experiments and models are separate entities that operate together in a simulation. Therefore, a model is the mathematical representation of a system with inputs and outputs, including its variables, parameters and states, regardless of knowns and unknowns. An experiment is a specific resolution of the model, which incorporates boundaries, initial conditions of states and algebraic variables (if any).

An important distinction to bear in mind is that models can be casual or acasual depending on the mathematical representation used. Casual modeling describes a system by ordinary differential equations (ODE) in explicit form, in other words, unknowns are directly derived from knowns. This is also termed as explicit modeling or block-oriented 
modeling. Simulink (MathWorks, 2020) is the most widespread tool of this kind to this date with a large distance with Trnsys (TRNSYS, 2020) standing in second place (Schweiger et al., 2020). In opposition, acasual modeling describes a system by differential algebraic equations (DAE) in implicit form, in other words, there is no directed resolution of the equations. Instead, the equations are worked out appropriately for each particular usage context or set of knowns, to compute the unknowns. This is also named as declarative or equation-based modeling. Modelica (Fritzson, 2014) is the most popular simulation language under the acasual umbrella with significant distance over Engineering Equation Solver (F-Chart Software, 2020) in second place (Schweiger et al., 2020).

In brief, acasual modeling brings more flexibility for utilization of models and modeling libraries towards multiple uses of a model. Refer to Schweiger et al. (2020), and the references therein, for a thorough discussion around casual and acasual modeling and simulation.

In general terms, the motivations in favor of the implementation of simulation instead of conducting experiments on actual systems are:

- Experiments incur in higher costs and risks than simulation does, along with the possibility of simulation of hypothetical (i.e. inexistent) systems.

- The timescale of the dynamics of the real system limit the possibility of experimenting, for example when the scope is outer space systems (extreme slow dynamics) or subatomic systems (extreme fast dynamics).

- Central variables may be inaccessible in the real system, while in a simulation all variables are observed and can be controlled, if need be.

- Disturbances and second-order effects can be isolated in a simulation, providing a better understanding of the main effects. In other words, a simulation can be customized to address the study of a system with respect to primary effects, even without measurement disturbances. 


\section{A.1.2 OPTIMIZATION FUNDAMENTALS}

Optimization problems can contain continuous variables, discrete variables or both (mixed). A simplified breakdown of classes of optimization problems is shown in Fig. A1.1. The most general of these classes is the mixed integer nonlinear program (MINLP) of the form (A1.1):

$$
\min _{x, y} f(x, y)
$$

subject to $h(x, y)=0$,

$$
\begin{gathered}
g(x, y) \leq 0, \\
x \in \mathbb{R}^{n}, y \in\{0,1\}^{t},
\end{gathered}
$$

Where $f$ represents the objective function to optimize (e.g. cost, error, etc.), $h$ are the equations that describe the behavior of the system (e.g. material balances, energy balances), and $g \leq 0$ are inequalities that represent process specifications or constraints. Continuous variables are represented by real $n$-vector $x$ and discrete variables are represented by the $t$-vector $y$. Additionally, if functions $f, g$ and $h$ are linear, (A1.1) is a mixed integer linear program (MILP), if not, (1.1) is a MINLP. A problem comprising integer variables only is an integer program (IP).

A similar classification is used with problems without discrete variables (no $y$ ), breaking down as linear program (LP) and nonlinear program (NLP) classes. This distinction is important due to the fact that optimal solutions of LP problems are always global (i.e. no better solution is guaranteed within tolerance) and insensitive to any initial value used in the calculation. However, NLP present a more complex breakdown which includes differentiability and convexity. In short, differentiable (continuous first and second derivatives) convex problems have a guarantee that any local optimal solution is a global solution, as opposed to nonconvex problems that local and global solutions may be different. Nondifferentiable optimization problems present discontinuities in either their first or second derivatives, which leads to a complete different solution methods. I suggest 
review Biegler (2010, chap. 1) for a comprehensive introduction to process optimization (NLP-focused), optimization problems' properties and application in chemical processes.

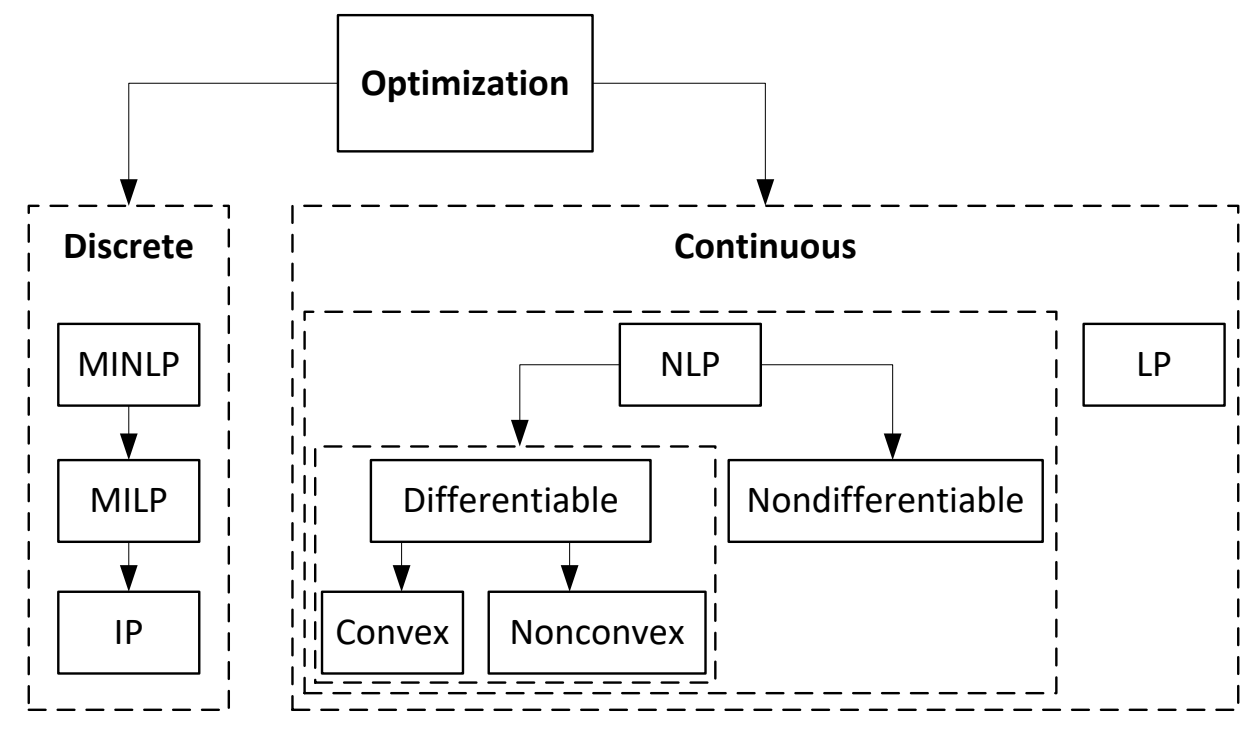

Fig. A1.1 - Classification of optimization problems. 


\section{APPENDIX B}

\section{B.1 OVERVIEW OF SIMULATION-BASED DECISION SUPPORT}

Simulation-based decision support tools have proven effective usefulness in process industry. Examples of application vary extensively across the process industry with respect to their specific scope of study. Moreover, several times simulation tools incorporate optimization capabilities to certain extent, being a combination of both what brings support to decision makers in the end. Hereinafter, I provide comments on some documented applications as examples of simulations underpinning decision making:

- Life cycle analysis - Halim and Srinivasan (2011) describe an innovative approach to the topic, implementing a knowledge-based simulation-optimization framework as decision support system to achieve process sustainability. In their work, an extensive use of process simulation is combined with multi-objective optimization using simulated annealing algorithm to deal with waste minimization of chemical processes. They demonstrate this framework on hydrodealkylation process and biodiesel production as case studies.

- Process flowsheet design - Ishii and Otto (2018), applied equation-oriented simulation integrated with sequential quadratic programming (SQP) to decouple highly nonlinear constraints and keep feasibility of solutions. Moreover, they describe a two-tier SQP method which successfully handles nonlinearities at simulation level maintaining feasibility and fast convergence. Their proposed method is demonstrated on largescale flowsheet design of distillation processes.

- Predictive simulation (example 1) - Fraedrich and Goldberg (2000), addressed the problem of simulation as a valid forecasting tool, proposing a five phases process in order to meet a validation specification. They start off by questioning if a simulation is actually subject to validation and progress further with a priori tests, design and execution of experiment, comparison with predictions, and lastly simulation improvement. 
- Process analysis - Suvarna et al. (2019), developed a first-principle dynamic model of bitumen mining and recovery process. This model is aimed at studying process variables which helps in forecasting process responses to changes and in-house training of operators.

- Process design (example 1) - Chen et al. (2015), apply simultaneous optimization combined with rigorous simulation to study heat integration in process plants. This framework allows use of simulations from different sources since it is based on derivative-free optimization algorithms and interfaces with Excel ${ }^{\circledR}$ or Python.

- Process design (example 2) - Yang and Feng (2019), present a simulation-based optimization method for synthesis of hydrogen networks with hydrogen sulfide $\left(\mathrm{H}_{2} \mathrm{~S}\right)$ removal. This work takes advantage of rigorous simulation and integrates the simulation results into hydrogen network superstructure used at optimization level.

Essentially, simulation-based tools tend to be case specific rather than general. Nonetheless, most examples would use simulation as a means to support rigorous mathematical modeling (Chen et al., 2015; Halim and Srinivasan, 2011; Yang and Feng, 2019) and forecasting complex processes (Galan et al., 2019a; Pablos et al., 2019; Suvarna et al., 2019). Most likely these features will be combined with one or more optimization techniques (Biegler et al., 2002), which in the end help making decisions towards process operation and design.

\section{B.1.1 HIGHLIGHTS OF SIMULATION-BASED METHODS}

General highlights of simulation-based decision support tools are:

- Forecasting capability, especially when rigorous modeling is required. This is the most distinctive feature of simulation over pure optimization. Moreover, simulation of scenarios is extensively used in industry when evaluating different process conditions e.g.: What-if analysis.

- Ease of modeling and results clarity, especially valued when the user is not a subject matter expert (SME) in the field of application of the simulation or is not familiar with 
the model itself. Typically, simulation tools provide a fairly straightforward human machine interface (HMI) where the end user is capable of making his way to reports and model details. In this regard, optimization-based tools are weaker than simulation. To the best of my knowledge, the former require explicit problem formulation which most end users are not able to figure out independently.

- Computing efficiency, as current state-of-the-art differential-algebraic equations (DAEs) solvers, e.g. IDAS (Serban et al., 2019), are capable of solving large equation systems with ease (Schweiger et al., 2020; Stechlinski et al., 2018).

\section{B.1.2 Challenges}

Current examples of challenges of simulation techniques are related to cosimulation, as a form to improve integration across different simulation tools efficiently. This topic is addressed in detail by Heinzl et al. (2018), presenting co-simulation use in an industrial energy assessment case study. Other authors focus their research on solvability and integration of DAEs with discrete events such as Stechlinski et al. (2018) with their contributions towards computationally efficient methods for nonsmooth DAEs (i.e. continuous and nondifferentiable). In particular, the efficient integration of dynamic simulation environments with optimization capability with and without discrete events is still a major open research field. Another approach for incorporation of discrete variables in models is by using logic functions such as disjunctions, Chen and Grossmann (2019) describes this methodology and its application on process industry case studies. In the end, these improvements would make simulation tools more useful across process operations and optimization (Navarro and Vassiliadis, 2014). Although it is important to acknowledge challenges and open issues in simulation-based applications, these are mentioned for the sake of contextualization and are not explored further. 



\section{APPENDIX C}

\section{C.1 NOTES ON OPTIMIZATION-BASED DECISION SUPPORT}

Optimization has been core to the process industry since many years ago, while increasing its role in process design and operation as computers became available (Grossmann and Biegler, 1995; Sargent, 1967). Likewise, optimization-based decision support tools have been present in various forms depending on the type of problem they are designed to tackle. Hereafter, I provide a brief review on the role and state-of-the-art of optimization in process operations and control, which is aimed at giving a broad overview of both topics. Although this is not an extensive review, I point out the most remarkable breakthroughs in key areas of optimization research and implementation across the process industry. It should be noted that only some topics amongst those reviewed are further developed in subsequent sections in accordance with the thesis objectives.

\section{C.1.1 PROCESS OPERATION}

Optimization comes very natural in process operations such as scheduling and planning, since decisions on resource allocation, delivery of raw materials, inventories management, meeting products demands, compliance with specifications and so on, are addressed and propagated down in the process decision pyramid (Fig. 1.1). On this respect, the PSE community contributed greatly towards better decision-making both on scheduling and planning (Grossmann and Biegler, 1995; Ignacio E. Grossmann and Harjunkoski, 2019; Grossmann and McDonald, 2004).

Generally speaking, state-task-network (STN) methodology (Kondili et al., 1993) was a major breakthrough back in the 1990s. Its importance relies on a framework development that allows for complex network configurations, while it is a discrete time model formulated as an MILP. This framework was further extended as resource-task- 
network (RTN) by Pantelides (1994), proposing resource balances across a network which leads to more compact MILP models. Both methods represent a significant share of techniques used to address scheduling problems in the process industry.

A thorough review on optimization techniques of scheduling problems and their structure is addressed by Méndez et al. (2006). Although the authors focus on short-term batch scheduling, some thoughts and challenges over more general longer term scheduling are provided. In particular, scheduling integration with planning and design are identified as promising and challenging for further research efforts. More recently, Harjunkoski et al. (2014) presented an extensive review on scheduling modeling and solving methods suitable for process industries, while Harjunkoski (2016) discusses actual deployment of scheduling solutions in industry. The authors discuss challenges to bridge the gap between academic solutions and industrial applicability from a technical viewpoint, identifying the following open issues: integration of scheduling across operation decision levels effectively, aim at ease-of-use applications and take into account model reformulations and re-usability, among others.

\section{C.1.2 Process ConTRoL}

Optimization techniques have been applied successfully on process control problems for several decades (Grossmann and Biegler, 1995; Grossmann and Harjunkoski, 2019), mostly in the form of model predictive control (MPC) or real-time optimization (RTO). González-Martín and Morilla (2019) demonstrate the implementation of closedloop economic optimization-based control in a Gasoline rundown blending system which has been successfully deployed in a crude oil Refinery, highlighting that ease-of-use is required for an implementation to succeed at industrial level. Additionally, early utilization of optimization methods included synthesis of control structures, so called Feedback Optimization Control, presented by (Morari et al., 1980; Morari and Stephanopoulos, 1980), their work aimed at determining manipulated variables and measured variables across units and plant-wide in a systematic and structured manner (Stephanopoulos and $\mathrm{Ng}, 2000)$. This approach was fundamental to developing self-optimizing control by 
Skogestad (2000) among other interesting ideas on optimizing processes by feedback control surveyed by Engell (2007).

\section{C.1.2.1 ABOUT MODEL PREDICTIVE CONTROL}

MPC is a form of control in which the current control action is obtained by solving on-line, at each sampling time, a finite horizon open-loop optimal control problem, using the current state of the plant as the initial state. The result of the optimization is an optimal control sequence and the first control action in this sequence is applied to the plant. For general introduction on MPC fundamentals and reviews I would recommend the following literature: Camacho and Bordons (2007), García et al. (1989), Lee (2011), Mayne et al. (2000), Morari and Lee (2014).

MPC was introduced, almost simultaneously, by Richalet et al. (1978) with their IDCOM software and model predictive heuristic control, then by Cutler and Ramaker (1979) with the dynamic matrix control (DMC) algorithm and developed even further later by Cutler (1983) in his PhD thesis. Gillette and Prett (1979) presented a MPC implementation on a fluidized catalytic cracking case study. The key idea of MPC was to optimize the control actions along a receding horizon such that desired reference trajectories of controlled variables are optimal (Fig C1.1). This approach was transformative in the field of advanced control in the process industry at the same time that gained popularity in the academic sector (Lee, 2011; Morari and Lee, 2014). In particular, generalized predictive control (GPC) first introduced by Clarke et al. (1987), and prediction self-adaptive control developed by De Keyser and Van Cauwenberghe (1985, 1981). These are predictive control examples that picked up the main ideas from earlier MPC algorithms and received great attention of the academic domain. García et al. (1989) surveyed successful industrial MPC deployments by the late 80 s including a summary of the main algorithms and software available, just about a decade after the DMC and IDCOM were first introduced. Additionally, Lundström et al. (1995) point out DMC limitations as poor performance for disturbance affecting plant inputs and poor robustness in plants with strong interactions, they propose a new MPC algorithm to 
overcome those limitations. Mayne et al. (2000) contributed establishing theoretical conditions for stability not addressed before. In overall, MPC has been a fertile research field with an ever growing list of industrial applications reported (Lee, 2011; Morari and Lee, 2014).

Tran et al. (2005), propose the use of first-principles nonlinear models to produce steady-state gains, instead of traditional step-response test for DMC-based control applications in a crude distillation unit complex at a Petronas refinery in Malaysia. A natural step forward from MPC was nonlinear MPC (NMPC), which essentially deals with nonlinear systems applying the same core ideas of MPC. In this respect, Allgöwer et al. (1999) give a theoretical overview of NMPC and its dual for state estimation, known as moving horizon estimation (MHE). For $\mathrm{MHE}$, a general theoretical review is described by Hedengren and Eaton (2017) and Rao and Rawlings (2000), both providing thorough analysis of the topic.

Biegler (2010, 2000) describes algorithms applied on nonlinear programming (NLP) and dynamic optimization (Biegler et al., 2002), one of his group's main contributions has been on the development of IPOPT (Wächter and Biegler, 2006) algorithm (an interior point method) which is the state-of-the-art of large scale NLP solvers. This method led the way for further progress on NMPC, with faster computation times and more industrial implementation. In this regard, Zavala and Biegler (2009) present a thorough study on optimality, stability and robustness of a so called advanced-step NMPC controller which essentially takes advantage of sensitivities computed by the NLP solver to reduce online computational time. Likewise, this has been implemented on MHE with success (Alessandri et al., 2010; Zavala et al., 2008). More recent works of Biegler and Thierry (2018) and Thierry et al. (2018) are focused on license-free software tools development aimed at easing NMPC and MHE formulations embedding NLP sensitivity with advancedstep strategy for fast computational times.

Other productive research fields within MPC have been: stochastic MPC (Heirung et al., 2018), multi-stage MPC (Lucia et al., 2013; Lucia and Engell, 2015), and robust MPC 
first introduced by Campo and Morari (1987). Basically, all these are approaches that account for explicit uncertainties in the process systems, while standard NMPC may only consider those as disturbances within a deterministic model.

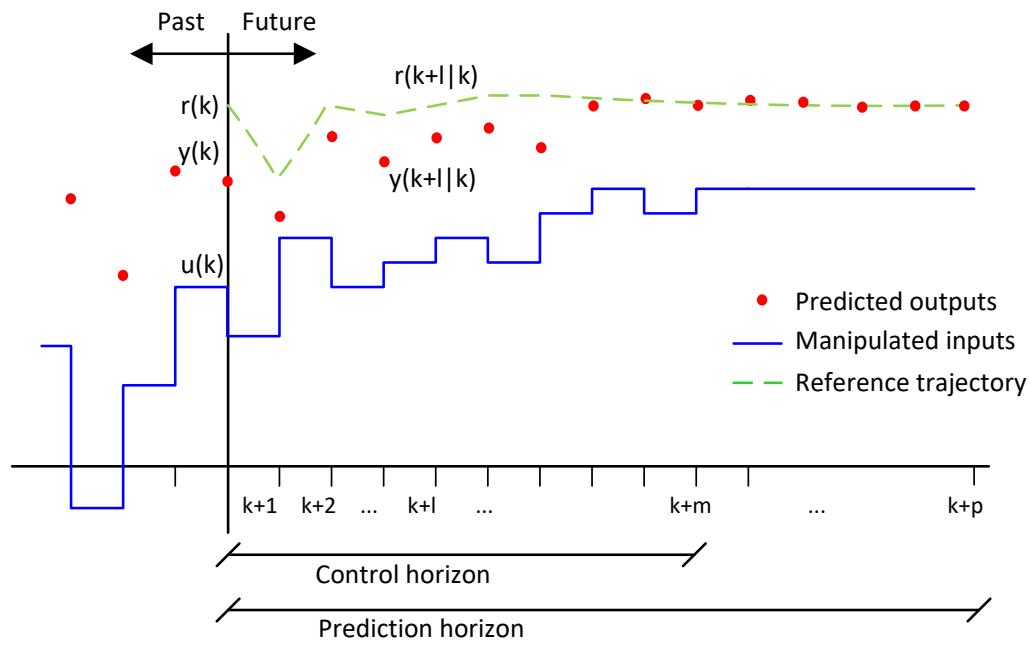

Fig. C1.1 - Model predictive control (MPC) simplified formulation. Manipulated inputs (u) along a control horizon of $m$ discrete-time steps are worked out such that, the predicted outputs ( $y$ ), and the reference trajectory $(r)$ have a minimum difference along the prediction horizon of $p$ discretetime steps.

\section{C.1.2.2 ABOUT REAL-TIME OPTIMIZATION}

Real-time optimization (RTO) has been a vigorous research field since the 1980s, almost alongside MPC, although it is not as spread in industry as MPC due to higher modeling requirements, the need of plant steady-state operation to successfully update calculations, among other reasons. RTO may be described as a supervisory control scheme, based on steady-state process models, which is used to determine optimal setpoints of manipulated variables such that the process economy is optimized and process constraints satisfied. Darby et al. (2011) provide an insightful overview and assessment of documented RTO practices which mostly remain valid today, the analysis is primarily focused on RTO-MPC arrangements since these have been the most popular (see Fig. C1.2). A comprehensive and more recent summary of RTO current methods including an actual process case study implementation is presented by de Prada and Pitarch (2018). 
Another approach on process optimization uses appropriate selection of control structure along with RTO (without MPC) to achieve maximum process profitability, this has been well discussed by Engell (2007).

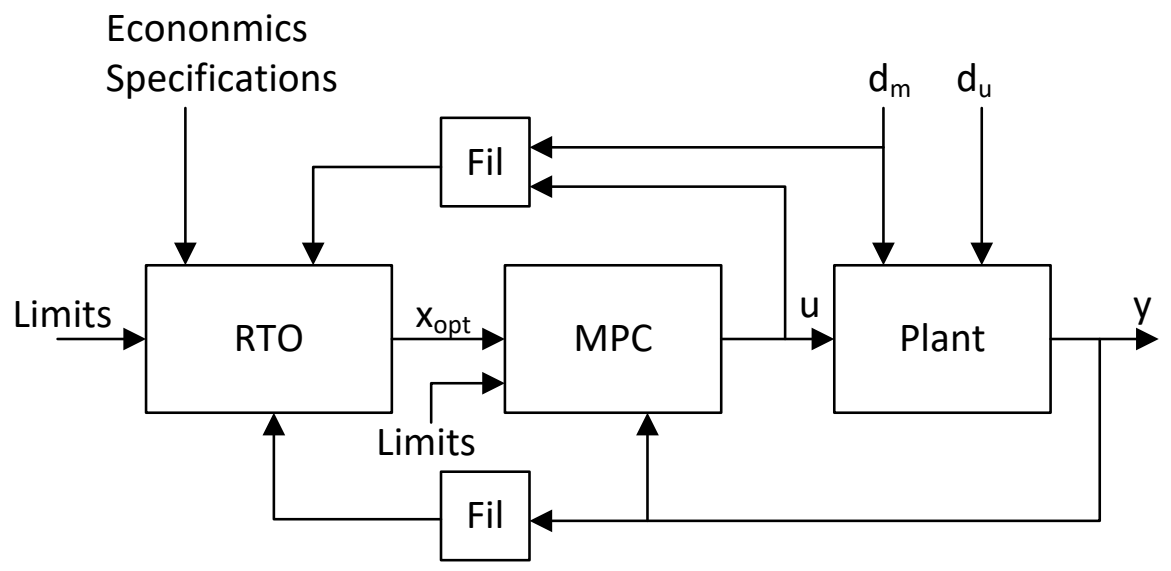

Fig. C1.2 - Simplified block diagram. Fil: filtering of signal. $d_{m}$ : measured disturbances. $d_{u}$ : unmeasured disturbances. $u$ : input variables. $y$ : output variables. $x_{\text {opt }}$ : RTO optimal decisions that are passed on to MPC.

Typically, RTO applications carry out multiple functions resulting in optimal setpoints (SP) to be passed on to controlled variables (CV) of MPCs underneath in the control structure (see Fig. C1.3). In brief, the first function is steady-state detection required to validate reasonable steady-state conditions of the process, this determines whether the rest of the functions are undertaken or not. If steady-state is validated, data treatment of all process data is performed, primarily gathering raw values, standard deviation figures and even essential logical functions (e.g. valve positions, flow directions in bidirectional lines) to help understand the actual condition of the process. These results feed the data reconciliation problem, aimed at reconciling process data of multiple source producing process values and parameter estimations that are consistent with the plant model. It is not until the last stage of the RTO, where the reconciled process data is used as input for running an optimization, which works out optimal set-points of process variables and gives steady-state set-points to CV in the MPCs. In addition, the RTO produces useful 
information such as up-to-date model parameters and operating performance figures, which are fed into upper hierarchy levels as shown in Fig. C1.3.

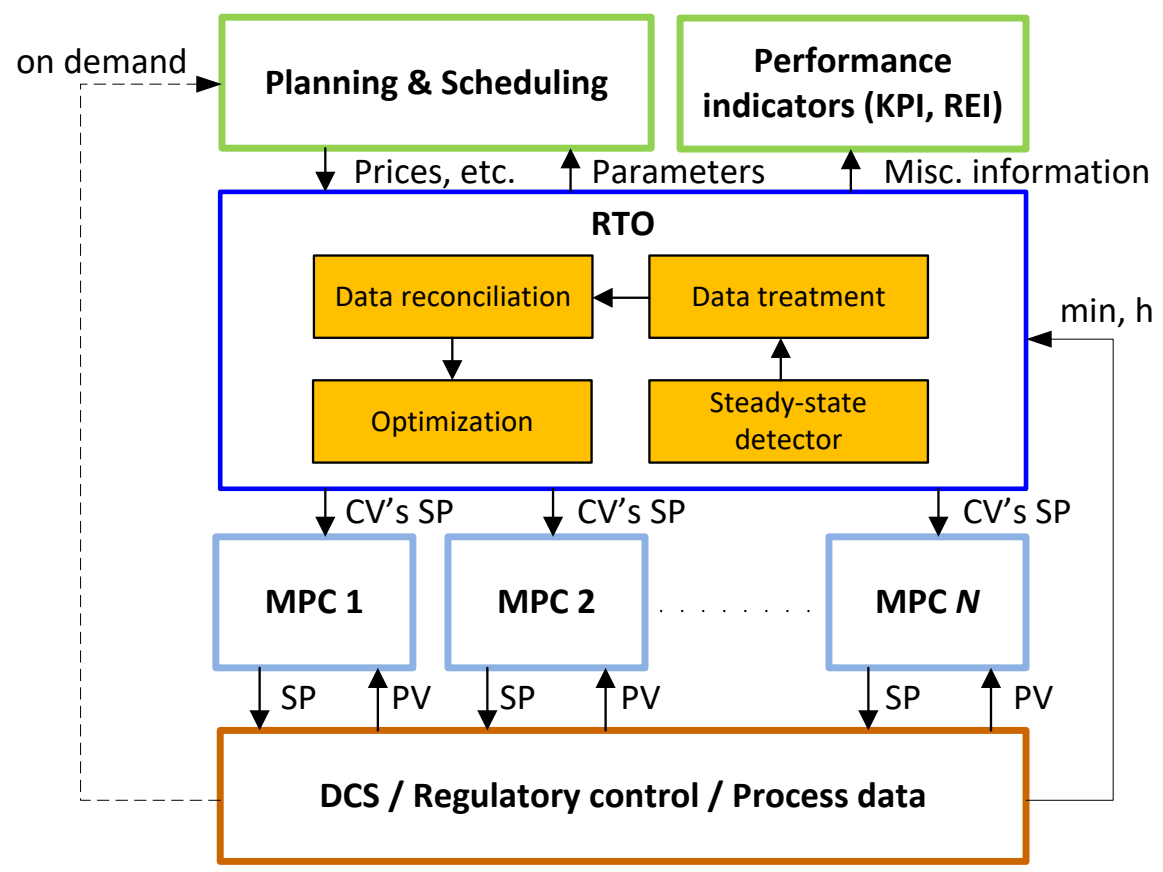

Fig. C1.3 - Typical architecture of an RTO and its information exchange with Planning and Scheduling (upper hierarchical level) and N MPC modules (lower hierarchical level), and process data updates. CV: controlled variables. SP: set-points. DCS: distributed control system. PV: process values. KPI: key performance indicator. REI: resource efficiency indicator.

Currently, there is important interest on MPC and RTO integration, either as a whole dynamic RTO (Aho et al., 2009) or as an economic MPC (Amrit et al., 2013; Ellis et al., 2014; Ellis and Christofides, 2015), or separately with focus on robust and stable integration (Alvarez and Odloak, 2010; De Souza et al., 2010). Ideas of the incorporation of economic objective functions into the MPC formulation emerged in the 90s as nonlinear MPC flourished in the research community. For some early applications using economic objectives in an MPC formulation see (de Prada and Valentín, 1996; Forbes et al., 1992; Forbes and Marlin, 1994; Gonzalez Santos et al., 2001; Young et al., 2002). I would suggest read (Amrit et al., 2013; Ellis et al., 2014; Ellis and Christofides, 2015) for tutorial and thorough reviews on economic MPC / dynamic RTO, their methods and challenges. 
Other researchers propose hybrid RTO alternatives to overcome the steady-state requirement for updating RTO solutions using transient plant measurements, given the fact that actual process units struggle to meet steady-state criteria (Krishnamoorthy et al., 2018). Srinivasan (2007), proposed an innovative gradient estimation technique using multiple units data for updates. Similarly, modified adaptation techniques have been used to overcome plant-model mismatch and structural uncertainty in RTO applications, some interesting case studies are described by Rodriguez-Blanco et al. (2015) and RodríguezBlanco et al. (2019). Additionally, stochastic dynamic optimization methods were analyzed for open-loop implementation on hydrodesulfurization plants showing promising results, however computational hurdles indicate that further refinement of this approach is required before it can effectively cope with real-time execution in a real plant environment (Navia et al., 2014).

Finally, open-loop strategies combined with online data reconciliation and optimization considering MPC restrictions have been deployed at industrial scale for a hydrogen network case study (de Prada et al., 2017), this work paved the way to further research on explicit account of process uncertainties by implementation of stochastic programming techniques (Galan et al., 2019b; Gutierrez et al., 2018).

\section{C.1.3 ChALlenges In INTEgRATING PROCESS OPERATIONS AND CONTROL}

The integration of process operations and control has been gaining attention, especially driven by: digital transformation, ever increasing communications and rapidly changing market demands that require assertive decision-making across all operation hierarchies. In this regard, I revisit inspiring works authored by Daoutidis et al. (2018) and Biegler (2018) and references cited in those papers, essentially around challenges and open issue towards successful integration of process operation and control.

The development of efficient surrogate process models, along with successful solution strategies, might well help in overcoming challenges posed by nonlinear dynamic optimization problems on detailed large-scale process models. This surrogate models 
should represent with enough level of detail key plant phenomena in scheduling, optimization and control layers (Cozad et al., 2014; Pitarch et al., 2019). However, in practice it might be necessary to tailor models in order to meet each layer particularities, instead of relying on one model alone. This can be achieved by implementing convex model approximations at higher levels in the control pyramid and nonconvex ones while it goes down into supervisory control. In this approach, consistency across models is of utmost importance. I firmly believe that, better results are more likely when consistency is addressed right from the conceptualization stage of the whole solution framework, rather than at later stages in the project.

Another significant challenge in the integration of process operations and control is the need of discrete decisions and sequential interlocks to be incorporated into MPC applications. In this point, both computational and theoretical hurdles arise, as determining close-loop properties for stability and developing hierarchical MPC frameworks is not well understood yet (Daoutidis et al., 2018a). These fully integrated schemes may well use a combination of first-principles, surrogate models and mixed integer dynamic optimization (MIDO), which itself poses a challenge, especially at industrial level. Biegler (2018) presents some interesting ideas and an industrial case study of a polymer plant, which is used to demonstrate how scheduling and dynamic optimization can be embedded successfully along with the significant savings unleashed by this innovative approach. Another approach is presented by Burnak et al. (2019) applying multiparametric programming to effectively integrate design, scheduling and control problems. However, to the best of my knowledge, this method has not been exercised on actual industrial problems which might be critical to better analyze its capability in the future.

It is generally accepted that in order for production management and control to integrate efficiently scheduling and supervisory control layers become critical (Daoutidis et al., 2018a; Engell and Harjunkoski, 2012). Moreover, successful solutions are likely to pose technical and personnel challenges. The former requires long time horizons, suitable for 
scheduling, with higher real-time execution frequencies to accommodate optimal supervisory control, while considering process economy, safety and control stability (Baldea and Harjunkoski, 2014). In addition, how best address uncertainty is an open issue going forward (Engell and Harjunkoski, 2012), Dias and lerapetritou (2016) present a very interesting review of this topic alongside its challenges. Personnel challenges are related to the fact that scheduling and control are usually different groups within an organization, these with variable interaction degrees depending on individual backgrounds, training and performance indicators (Shobrys and White, 2002).

\section{C.1.4 ENTERPRISE-WIDE OPTIMIZATION}

Enterprise-wide optimization (EWO) is a discipline within process systems engineering that is concerned with the optimal coordination of the operations of supply chain, such as supply material, manufacturing operations and distribution of products (Grossmann, 2005; Wassick, 2009). Although EWO has gained significant interest in the academic and industrial domains in the last two decades, it is a relatively new research field within decision-making sciences that lies in between of process systems engineering and operations research. In fact, I would suggest review Grossmann (2014) for an EWO introduction and state-of-the-art review, and Papageorgiou (2009) for reviewing supply chain optimization challenges. In the following paragraphs I briefly revisit and point out a handful of key EWO articles focusing on the optimization methods developed therein and challenges faced.

Naturally, EWO integrates all hierarchy levels of decision-making from strategic (long term), passing through tactical (medium term) and operational (short term) (Papageorgiou, 2009). The first two, present a spatial integration problem, since it requires coordination of tasks across different sites and subsystems within an organization. I would suggest an insightful review article by Shapiro (2004) as it gives a provocative viewpoint on the challenges faced in strategic supply chain modelling. The operational level, requires efficient temporal integration to reflect decision-making at different timescales such as scheduling and control (Grossmann, 2005). 
In overall, challenges in EWO may be categorized as follows (Grossmann, 2005, 2014):

- The modeling challenge, dealing with effectively capturing tasks across planning, scheduling, operations at each site, most likely incorporating nonlinear models. This requires adequate mathematical programming along with logic-based models to accurately reflect decision-making activities across the supply chain (Misener et al., 2011; Mouret et al., 2009). These problems are modelled with multiperiod mixedinteger NLP or LP (Trespalacios and Grossmann, 2014).

- The multiscale optimization challenge, which takes on the coordination across different timescales over a given time horizon, going from days to years in order to incorporate strategic, tactical and operational decision-making levels. Decomposition techniques are specially used to address this issue, since these have demonstrated good performance in working out across spatial and temporal scales. Additional references on this point are provided in brackets (Allgor and Barton, 1999; Burnak et al., 2019; Muñoz et al., 2011; Shobrys and White, 2002).

- The uncertainty challenge, which refers to explicit accountability of stochastic nature of random events realizations such as demands, malfunctions, prices. In this respect, efficient stochastic programming techniques should be developed enough to cope with ever increasing requirement of uncertainty incorporation in EWO decisionmaking solutions. Additional references on this point are provided in brackets (Linderoth and Wright, 2003; You et al., 2009).

- The algorithmic and computational challenges, deal with the fact that the previous three require efficient algorithms that take into account modern computational advances as distributed or grid computing. Additional references on this point are provided in brackets (Daoutidis et al., 2018b; Goux and Leyffer, 2002).

As an illustration of spatial integration complexity, an interesting case study of integration of a chemical complex with multi plant and multiproduct is presented by Wassick (2009), this was successfully solved based on the resource task network method. 
Terrazas-Moreno et al. (2010) developed bi-criterion formulation for optimizing the design of an integrated site considering random failures among other uncertainties. They got promising results, however the exponential growth in problem size as the number of scenario increases was identified as an area for further improvement of their approach.

Demand side management (DSM) has been growing as a research topic in the process systems engineering community in the last decade, mainly due to increased availability of multiple energy providers as renewable energies spread across power grids. Zhang and Grossmann (2016) describe the problem fundamentals, perspectives and challenges from a EWO standpoint.

For further references to case studies and methods see Grossmann (2014) and the literature therein. 


\section{APPENDIX D}

\section{D.1 Placement REPORT}

Hereinafter I reproduce the placement report where the main aspects of the industrial work of this thesis are informed. The following is a verbatim copy of the original report submitted to the PRONTO (https://www.h2020pronto.eu/) project coordinator for further reporting to the European Commission.

\section{D.1.1 INTRODUCTION}

\section{D.1.1.1 PlaCEMENT BASIC DATA}

This report describes the placement of Aníbal Galán, Early Stage Researcher $M$ (ESR-M) employed by University of Valladolid within PRONTO project, at Petronor's refinery in Muskiz, Spain. The placement started on 24 ${ }^{\text {th }}$ January 2017 and finished on $9^{\text {th }}$ November 2018, with an interruption of three months ( $5^{\text {th }}$ March 2018 to $6^{\text {th }}$ June 2018) due to an overlapping of ESR secondment at Carnegie Mellon University in Pittsburgh, USA. In total, the placement was 18.8 months (considering 30 days per month). At Petronor ESR-M was hosted by the department of advanced control and optimisation (ACO), under the direct supervision of Rafael González. Regular follow-up meetings with Rafael and César De Prada (academic supervisor were held during the placement where research project progress was discussed, and decisions were made regarding any issues that arouse. In addition, ESR participated in the ACO group weekly meetings which helped him to get insights of multiple refinery topics.

\section{D.1.2 HYDROGEN NETWORK CASE STUDY}

Refinery hydrogen $\left(\mathrm{H}_{2}\right)$ network is required to provide $\mathrm{H}_{2}$ for sulfur removal of hydrocarbons $(\mathrm{HC})$ to produce diesel and gasoline fuels. Sulfur removal occurs in different types of hydrodesulfurization process units throughout the network. And $\mathrm{H}_{2}$ is supplied through hydrogen process units (HPU). Therefore, maximum benefit is obtained by 
processing maximum $\mathrm{HC}$ loads at minimum $\mathrm{HPU}$ costs (i.e.: minimum $\mathrm{H}_{2}$ production). However, due to the facts that in reality processes are not static, and plant measurements may not collect enough reliable information for the operators to make sensible decisions, computer-based tools become critical for operation of the network. For instance, realtime optimisation (RTO) applications and predictive simulation tools taking account uncertainties from machinery condition and the process could prove of key importance in the decision-making process at all level.

\section{D.1.1.2.1 PETRONOR HYDROGEN NETWORK}

The refinery hydrogen network comprises 18 process units, four producers and fourteen consumers; a simplified schematic of the network is presented in Fig. D.1 (Gomez, 2016). Operation decisions are made at different levels depending on the time span, this follows the ISA (2010A; 2010B, 2012, 2013A, 2013B) automation pyramid of control, from planning and scheduling to control room and field controllers. Figure D.2 shows a simplified control scheme of the $\mathrm{H}_{2}$ network, and the different levels integrated. A commercial dynamic matrix controller (DMC) manages the network in real time, controlling the six process units with the highest $\mathrm{H}_{2}$ demand. At the same time, a prototype RTO computes reconciliation and optimal distribution for the whole network and passes those results to the plant information system. Therefore, long-run $\mathrm{H}_{2}$ management policies are shown to operators from the RTO. It must be born in mind that this RTO was to be commissioned when the placement started.

Based on previous data reconciliation techniques applied by Sarabia et al. (2012) raw data from the process network are validated.

In brief, $\mathrm{H}_{2}$ network management involves decisions at many levels, and especially when considering uncertainties due to plant measurements and equipment, the decisionmaking process grows in complexity. Therefore, computer-aided process tools for decision support play a key role in efficient network management by pointing out the right direction in which to direct the process, given certain economic and process criteria. 


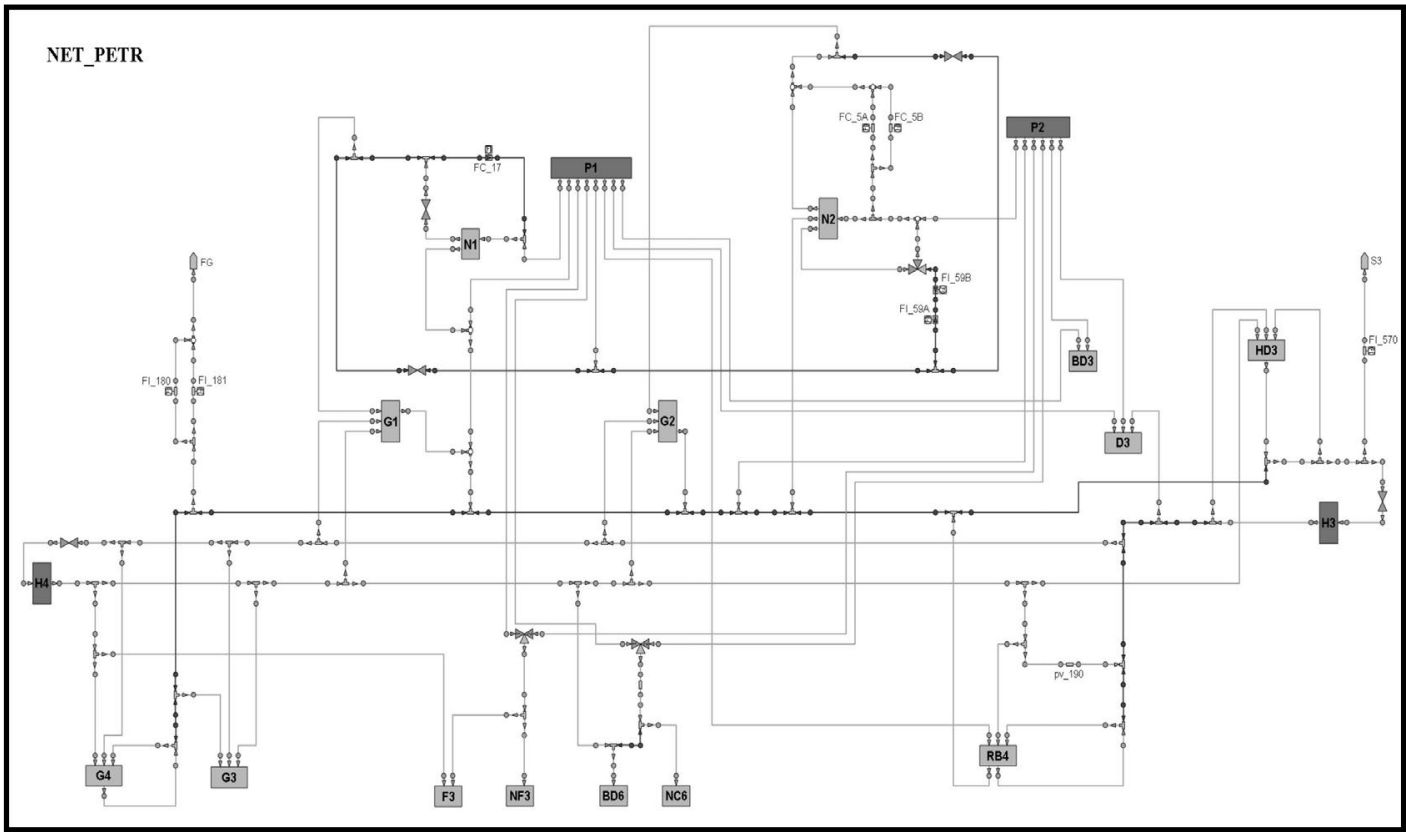

Fig. D.1. Schematic of Petronor hydrogen network. Source: Gomez (2016).

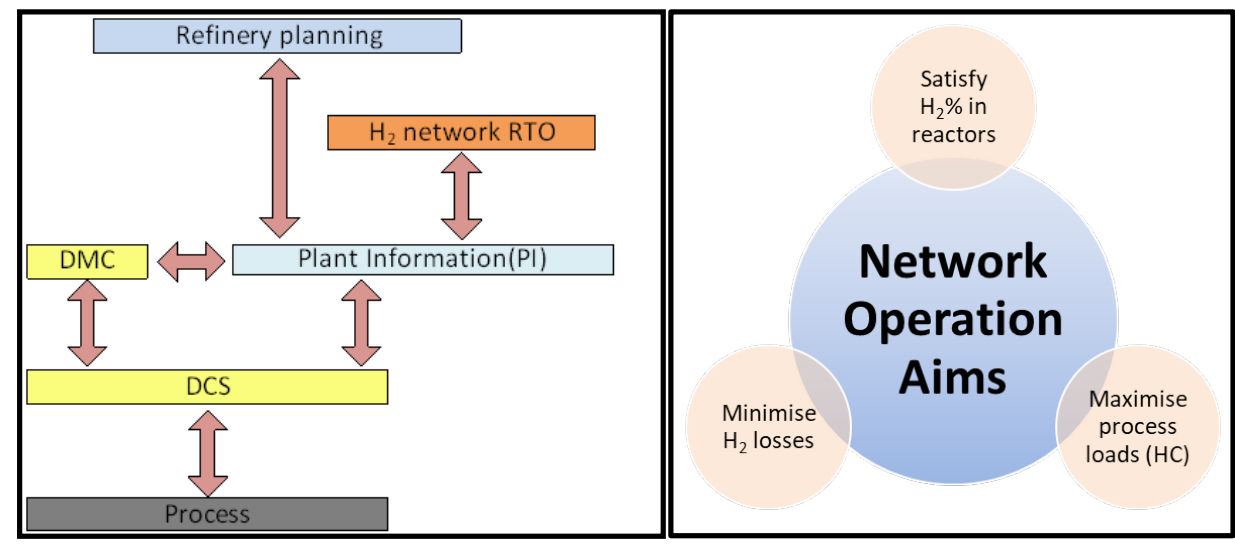

Fig. D.2. Simplified control levels integration scheme.

\section{D.1.1.3 PURPOSE OF PLACEMENT}

The placement at Petronor had several aims pointed out hereinafter.

\section{D.1.1.3.1 NETWORK OPERATION DETAILS}

Given the major project aim of providing meaningful information and suggestions to operators, namely predictive simulation, it was critical to know in detail the operation 
policies and control of the network. These should be surveyed and discussed at first-hand with refinery personnel at all levels, from operators to managers.

\section{D.1.1.3.2 DATA COLLECTION}

Another important task of the placement was related to data collection from several sources, including process plant data, such as flowrates, temperatures, and design or equipment specific data. The former is typically available from plant information systems, and pipe and instruments diagrams. The latter obtained from process books, operation manuals and vendors data books. Under data collection it is included any plant tests carried out for modelling purposes. For instance, plant equipment as gas purification membranes was to be modelled from plant tests.

\section{D.1.1.3.3 DECISION-MAKING INSIGHT}

In order to develop decision support tools, an in depth understanding and engagement with the decision-making process at its various levels was important. Therefore, during my placement I strove to gain insightful knowledge of Petronor's decisions process with an impact on the $\mathrm{H}_{2}$ network, either direct or indirect.

\section{D.1.1.4 REPORT OUTLINE}

The rest of the report presents the expectations of the placement (section A.2) and then describes the technical challenges of the research (section A.3). The next section is an identification of new skills sets developed at Petronor (section A.4), followed by the description of access to new technologies (section 5). Finally, it finishes with a section of suggestions for future collaboration (section 6) and conclusions (section 7).

\section{D.1.2 EXPECTATIONS BEFORE THE PLACEMENT}

ESR-M started the placement after six months of joining PRONTO, therefore most of the expectations were in line with the research plan at that point and throughout the following 18 months. A summary is presented in Table D.1, along with an expectations' fulfilment degree. 
Table D.1 - ESR-M technical expectations before the placement and their fulfilment degree.

\begin{tabular}{|l|l|l|}
\hline Topic & Description & Fulfilment \\
\hline Dynamic simulation model & $\begin{array}{l}\text { Development of dynamic libraries capable } \\
\text { of representing actual } \mathrm{H}_{2} \text { network plants. }\end{array}$ & $100 \%$ \\
\hline Prototype improvement & $\begin{array}{l}\text { Commissioning and validation of RTO as } \\
\text { decision support tool. }\end{array}$ & $100 \%$ \\
\hline Plant tests & $\begin{array}{l}\text { Design and carry out plant tests to improve } \\
\text { model accuracy. }\end{array}$ & $80 \%$ \\
\hline Decision-making process & $\begin{array}{l}\text { Understand and engage the decision- } \\
\text { making process regarding } \mathrm{H}_{2} \text { management } \\
\text { at all levels. }\end{array}$ & $100 \%$ \\
\hline On-site training & $\begin{array}{l}\text { Tailored hands-on training, especially on } \\
\text { process control used in refineries. }\end{array}$ & $100 \%$ \\
\hline
\end{tabular}

The following subsections expand the descriptions of each expectation topic.

\section{D.1.2.1 DYNAMIC SIMULATION MODEL}

Development of dynamic libraries and simulation models able to represent the $\mathrm{H}_{2}$ network were a milestone of the placement. While at Petronor the ESR expected to rather than building up models of the plant, testing and presenting them to operators at actual plant conditions. This expectation was fully accomplished.

\section{D.1.2.2 PROTOTYPE IMPROVEMENT}

Previous research delivered a prototype RTO which I expected to commission and validate in collaboration with in-house technical staff and colleagues of University of Valladolid. This tool was the starting point to other research lines such as two-stage stochastic programming and mixed integer nonlinear formulations of the . This expectation was fully accomplished.

\section{D.1.2.3 Plant TESTS}

The ESR expected to carry out some plant test to gather critical equipment responses to different inputs. These supported model improvements that later on demonstrated their usefulness in getting meaningful operation long-run policies. Its 
fulfilment was not 100 percent (Table D.1), due to plant operation conditions that were required for some final tests.

\section{D.1.2.4 DECISION-MAKING PROCESS}

Identification of information gaps were expected at all levels in the decision-making process. From those the ESR-M could define exactly which information was missing, and figure out how to bridge the gaps in between. This expectation was fully accomplished.

\section{D.1.2.5 ON-SITE TRAINING}

Expectations on refinery advanced control practices were present. In particular, identification tests, DMC configuration, and inference models, were planned and fulfilled. This expectation was fully accomplished.

\section{D.1.3 NEW SKILLS/ADDED KNOWLEDGE}

Throughout the placement the ESR-M developed new skills and enhanced some others with a first-hand insight into the refinery network practices. Table D.2 shows the most relevant skills with a short description of each.

Table D.2- ESR-M new skills set description, sorted by topic.

\begin{tabular}{|l|l|}
\hline Topic & Description \\
\hline $\begin{array}{l}\text { Dynamic simulation and } \\
\text { state estimation }\end{array}$ & $\begin{array}{l}\text { ESR-M surveyed and developed software libraries of process unit } \\
\text { models able to reflect actual plant dynamics, that can be used as } \\
\text { generic building blocks of tailored (or theoretical) refinery } H_{2} \\
\text { networks. In addition, dynamic state estimation libraries and its } \\
\text { usefulness to aid operators' decisions were developed during the } \\
\text { placement (Galan et al., 2017; Galan et al., 2019). }\end{array}$ \\
\hline Plant tests & $\begin{array}{l}\text { ESR-M designed and conducted plant equipment performance } \\
\text { tests. These were reported to Petronor and used to improve the } \\
\text { network model. An internal report to Petronor was delivered. }\end{array}$ \\
\hline $\begin{array}{l}\text { Results visualization and } \\
\text { reporting }\end{array}$ & $\begin{array}{l}\text { ESR-M designed and commissioned visualization of RTO and } \\
\text { reconciliation results. Concretely, 19 views were uploaded on to } \\
\text { PI }{ }^{\circledR} \text { (plant information system), and three automatic report } \\
\text { spreadsheets delivered to Petronor for their use in results } \\
\text { assessment. }\end{array}$ \\
\hline DMC ${ }^{\circledR}$ usage & $\begin{array}{l}\text { ESR-M gained hands-on experience in DMC plus application, a } \\
\text { commercial model predictive controller (MPC), with }\end{array}$ \\
\hline
\end{tabular}




\begin{tabular}{|c|c|}
\hline & $\begin{array}{l}\text { identification, configuration and simulation modules. An } \\
\text { assessment of potential expansion of current DMC scope was } \\
\text { performed and an internal report submitted. In addition, took a } \\
\text { DMC maintenance training given by a consultancy company on } \\
\text { site. }\end{array}$ \\
\hline $\begin{array}{l}\text { Hydrogen } \\
\text { operation }\end{array}$ & $\begin{array}{l}\text { ESR-M developed operational experience in network operation } \\
\text { from a control point of view. In addition, ESR received critical } \\
\text { inputs for the formulation of the stochastic optimisation problem } \\
\text { and the operation under degraded conditions. }\end{array}$ \\
\hline Hydrogen production & $\begin{array}{l}\text { ESR-M gained experience in } \mathrm{H}_{2} \text { steam reformers operation and } \\
\text { control, including emergency shutdowns and procedures. }\end{array}$ \\
\hline
\end{tabular}

\section{D.1.4 ACCESS TO NEW TECHNOLOGIES AND PROCESSES}

This was an industrial placement so there was a first-hand access to industrial assets and their operation personnel. This means access to, hydrogen production units (steam reformers and platformers), and desulfurization, and hydrocracking units.

\section{D.1.5 ACTIVITIES AND RESULTS}

ESR-M presented several conference papers during this placement. In addition, research progress and results were presented and reported within PRONTO and Petronor in various occasions. Table D. 3 describes the most relevant activities and their outcomes.

Table D.3 - Activities and results

\begin{tabular}{|c|c|}
\hline CONFERENCES & \\
\hline XXXVIII Jornadas de Automática JA2017 & $\begin{array}{l}\text { Galan, A., De Prada, C., Gutierrez, G., Sarabia, } \\
\text { D., González, R., 2017, Dynamic simulation } \\
\text { applied to refinery hydrogen networks. }\end{array}$ \\
\hline $\begin{array}{l}\text { European Symposium on Computer-Aided } \\
\text { Process Engineering (ESCAPE-27) }\end{array}$ & $\begin{array}{l}\text { Galan, A., De Prada, C., Gutierreza, G., Sarabia, } \\
\text { D., González, R., Sola, M., Marmol, S., Pascua, } \\
\text { C., 2017, Resource efficiency indicators } \\
\text { usefulness for decision-making process of } \\
\text { operators: refinery hydrogen network case } \\
\text { study. }\end{array}$ \\
\hline $\begin{array}{l}\text { 10th IFAC International Symposium on } \\
\text { Advanced Control of Chemical Processes } \\
\text { (ADCHEM) }\end{array}$ & $\begin{array}{l}\text { Galan, A., De Prada, C., Gutierrez, G., Sarabia, } \\
\text { D., Gonzalez, R., Sola, M., Marmol, S., 2018, } \\
\text { Validation of a hydrogen network RTO } \\
\text { application for decision support of refinery } \\
\text { operators. }\end{array}$ \\
\hline
\end{tabular}




\begin{tabular}{|c|c|}
\hline $\begin{array}{l}\text { 10th IFAC International Symposium on } \\
\text { Advanced Control of Chemical Processes } \\
\text { (ADCHEM) }\end{array}$ & $\begin{array}{l}\text { Gutierrez, G., Galan, A., Sarabia, D., De Prada, } \\
\text { C., 2018, Two-stage stochastic optimization of } \\
\text { a hydrogen network. }\end{array}$ \\
\hline $\begin{array}{l}\text { 12th IFAC Symposium on Dynamics and } \\
\text { Control of Process Systems, including } \\
\text { Biosystems (DYCOPS 2019) }\end{array}$ & $\begin{array}{l}\text { Galan, A., De Prada, C., Gutierrez, G., Sarabia, } \\
\text { D., Gonzalez, Predictive Simulation Applied to } \\
\text { Refinery Hydrogen Networks for Operators' } \\
\text { Decision Support (manuscript submitted) }\end{array}$ \\
\hline \multicolumn{2}{|l|}{ SECONDMENT } \\
\hline $\begin{array}{l}\text { ESR visited Carnegie Mellon University (CMU) } \\
\text { as secondment }\end{array}$ & $\begin{array}{l}\text { At CMU ESR completed the formulation of his } \\
\text { case-study with a two-stage stochastic } \\
\text { approach to allow explicit consideration of } \\
\text { process uncertainty. Details of outcomes were } \\
\text { presented in the secondment report. }\end{array}$ \\
\hline \multicolumn{2}{|l|}{ PRONTO } \\
\hline $\begin{array}{l}\text { Posters and presentation sessions in } \\
\text { PRONTO meetings }\end{array}$ & $\begin{array}{l}\text { Research progress and case-study } \\
\text { presentations. }\end{array}$ \\
\hline \multicolumn{2}{|l|}{ PETRONOR } \\
\hline $\begin{array}{l}\text { Presentations and reports on placement } \\
\text { progress }\end{array}$ & $\begin{array}{l}\text { Internal sessions and reports showcasing } \\
\text { placement progress, focusing on decision } \\
\text { support tools available for operators and their } \\
\text { visualisation. }\end{array}$ \\
\hline
\end{tabular}

\section{D.1.6 SUGGESTION FOR FURTHER RESEARCH AND COLLABORATIONS}

Petronor is usually very open to collaborate with research projects in areas where they see high potential for improvement. Basically, problems of: scheduling, blending, network management, conditioned-based monitoring, etc., seem to be of high interest from their point of view and academic research has plenty to contribute as well.

Another sort of collaboration ESR-M would suggest is related to: visualisation of results, automatic reports with summarised actions. That is how a software tool could address different users, and give them only the most significant information in order to point them in the right direction. Frequently, this is not the case and tools present large amounts of information, making users doubtful regarding concrete actions for them to take. 


\section{D.1.7 EVALUATION OF THE VALUE OF THE PLACEMENT}

ESR-M is convinced that one of the main advantages of PRONTO ITN is precisely the industrial placement. Particularly, ESR-M is grateful to Petronor personnel for their welcoming and supportive attitude towards him and his research project. In addition, acknowledges the support of his academic supervisor who he met mostly by videoconference for most of the placement with all the difficulties that this presents. Notwithstanding, a breakdown of benefits and challenges is presented in Table D.4 as an overview. In overall, the ESR-M evaluation of the placement is very positive.

Table D.4 - Benefits and challenges of the placement.

\begin{tabular}{|c|c|}
\hline Benefits & Challenges \\
\hline $\begin{array}{l}\text { 1. First-hand knowledge of potential end } \\
\text { users of predictive simulation tool. } \\
\text { 2. Engage with an actual process network } \\
\text { not devoted, primarily, to research } \\
\text { purposes, just production. }\end{array}$ & $\begin{array}{l}\text { 1. Mobility might be tedious, especially } \\
\text { during relocation periods, for instance } \\
\text { when changing residence before and } \\
\text { after placements since these may take } \\
\text { more than time than planned and } \\
\text { overlap with other commitments. }\end{array}$ \\
\hline $\begin{array}{l}\text { 3. Develop new skills based on actual } \\
\text { industrial practices. }\end{array}$ & $\begin{array}{l}\text { 2. Remote meetings with academic } \\
\text { supervisor }\end{array}$ \\
\hline $\begin{array}{l}\text { 4. Networking and exchange with } \\
\text { industrial practitioners. } \\
\text { 5. Enough time to actually blend in }\end{array}$ & $\begin{array}{l}\text { 3. Collaboration within consortium is very } \\
\text { hard if fellows do not coincide (or } \\
\text { overlap) their placements/secondments }\end{array}$ \\
\hline $\begin{array}{l}\text { another society, conduct research and } \\
\text { get results. } \\
\text { 6. Opportunity to understand gaps } \\
\text { between academic and industrial } \\
\text { sectors, and foresee alternatives to } \\
\text { bridge them over. }\end{array}$ & $\begin{array}{l}\text { 4. Housing overlapping (unavoidable during } \\
\text { relocation), and other relocation } \\
\text { expenses should be considered as } \\
\text { research cost and therefore not paid by } \\
\text { the ESR. }\end{array}$ \\
\hline
\end{tabular}

\section{D.1.8 ConCluSions}

The industrial placement gave ESR-M an outstanding opportunity of hands-on experience of refinery $\mathrm{H}_{2}$ network industrial practices, which aided him in defining his research project in detail and pinning down a concrete case-study. ESR-M stayed at Petronor refinery for almost 19 months, where he conducted research and got results that were presented both internally within Petronor and PRONTO, and externally in 
international conferences. During this period, ESR-M received feedback and support from: Pronto network, his industrial supervisor, and academic supervisor (remotely).

In overall, the evaluation of the placement is positive, being its most remarkable benefit the engagement with an actual refinery $\mathrm{H}_{2}$ network and how this contributed to the research project at many levels. Additionally, some challenges arose as well, such as difficulties related to the ESR-M mobility in the commencement and completion of the placement and residence overlapping (relocation). Those could be better addressed in future projects, for example assuming them eligible as research costs. Nonetheless, benefits largely outweighed challenges.

\section{D.1.9 REFERENCES}

Galan, A., De Prada, C., Gutierrez, G., Gonzalez, R. (2017). Dynamic simulation applied to refinery hydrogen networks, in: . Zenodo. doi:10.5281/zenodo.1013258

Galan, A., De Prada, C., Gutierrez, G., Sarabia, D., Gonzalez, R., Sola, M., Marmol, S. (2018). Validation of a hydrogen network RTO application for decision support of refinery operators, 10th IFAC Symposium on Advanced Control of Chemical Processes (ADCHEM 2018), in: . Zenodo. doi:10.5281/zenodo.1405654

Galan, A., De Prada, C., Gutierrez, G., Sarabia, D., Gonzalez, Predictive Simulation Applied to Refinery Hydrogen Networks for Operators' Decision Support, DYCOPS 2019 (manuscript submitted)

Gutierrez, G., Galan, A., Sarabia, D., De Prada, C., Two-stage stochastic optimization of a hydrogen network, 10th IFAC Symposium on Advanced Control of Chemical Processes (ADCHEM 2018)

Gomez, E., (2016). A study on modelling, data reconciliation, and optimal operation of hydrogen networks in oil refineries, Doctoral thesis, University of Valladolid, Spain 
ISA (2010A), ANSI/ISA-95.00.01-2010 (IEC 62264-1 Mod) - Enterprise-Control System Integration - Part 1: Models and Terminology, ISA

ISA (2010B), ANSI/ISA-95.00.02-2010 (IEC 62264-2 Mod) - Enterprise-Control System Integration - Part 2: Object Model Attributes, ISA

ISA (2012), ANSI/ISA-95.00.04-2012, Enterprise-Control System Integration - Part 4: Objects and attributes for manufacturing operations management integration, ISA

ISA (2013A), ANSI/ISA-95.00.03-2013 (IEC 62264-3 Modified) - Enterprise-Control System Integration - Part 3: Activity Models of Manufacturing Operations Management, ISA

ISA (2013B), ANSI/ISA-95.00.05-2013, Enterprise-Control System Integration - Part 5: Business-to-Manufacturing Transactions, ISA

Sarabia, D., de Prada, C., Gomez, E., Gutierrez, G., Cristea, S., Sola, J., Gonzalez, R., (2012). Data reconciliation and optimal management of hydrogen networks in a petrol refinery, Control Engineering Practice, Volume 20, Issue 4, pp 343-354, ISSN 0967-0661, https://doi.org/10.1016/j.conengprac.2011.06.009 